\title{
Automatic Trace-Based Performance Evaluation Model Building for Concurrent Distributed Systems
}

\author{
By
}

Ahmad Mizannojehdehi, MSc

A thesis submitted to the Faculty of Graduate and Postdoctoral Affairs in partial fulfillment of the requirements for the degree of

Doctor of Philosophy

in

Electrical and Computer Engineering

Ottawa-Carleton Institute for Electrical and Computer Engineering (OCIECE)

Department of Systems and Computer Engineering

Carleton University

Ottawa, Ontario, Canada, K1S 5B6

(C) 2013

Ahmad Mizannojehdehi 
Library and Archives

Canada

Published Heritage

Branch

395 Wellington Street

Ottawa ON K1A ON4

Canada
Bibliothèque et

Archives Canada

Direction du

Patrimoine de l'édition

395 , rue Wellington

Ottawa ON K1A ON4

Canada
Your file Votre référence

ISBN: 978-0-494-94541-4

Our file Notre référence

ISBN: 978-0-494-94541-4
NOTICE:

The author has granted a nonexclusive license allowing Library and Archives Canada to reproduce, publish, archive, preserve, conserve, communicate to the public by telecommunication or on the Internet, loan, distrbute and sell theses worldwide, for commercial or noncommercial purposes, in microform, paper, electronic and/or any other formats.

The author retains copyright ownership and moral rights in this thesis. Neither the thesis nor substantial extracts from it may be printed or otherwise reproduced without the author's permission.
AVIS:

L'auteur a accordé une licence non exclusive permettant à la Bibliothèque et Archives Canada de reproduire, publier, archiver, sauvegarder, conserver, transmettre au public par télécommunication ou par l'Internet, prêter, distribuer et vendre des thèses partout dans le monde, à des fins commerciales ou autres, sur support microforme, papier, électronique et/ou autres formats.

L'auteur conserve la propriété du droit d'auteur et des droits moraux qui protege cette thèse. $\mathrm{Ni}$ la thèse ni des extraits substantiels de celle-ci ne doivent être imprimés ou autrement reproduits sans son autorisation.
In compliance with the Canadian Privacy Act some supporting forms may have been removed from this thesis.

While these forms may be included in the document page count, their removal does not represent any loss of content from the thesis.
Conformément à la loi canadienne sur la protection de la vie privée, quelques formulaires secondaires ont été enlevés de cette thèse.

Bien que ces formulaires aient inclus dans la pagination, il n'y aura aucun contenu manquant. 


\section{Abstract}

Performance models can be used for analyzing and predicting performance behaviours of software systems. This prediction allows designing high performance software systems or locating performance bottlenecks of a deployed system. Despite its benefits, the creation of a performance model is not a trivial task since it requires the distillation of the performance related parameters of an application from its documentation, source code, or running behaviours. The creation of a performance model is even more difficult when the target is a distributed application where processing resources are geographically distributed and their only means of interactions is through passing of messages.

This research introduces WebTime which is a framework of tools, specifications and methodologies to trace and instrument the events of a distributed application and to automatically construct its layered queuing network (LQN) performance model. WebTime uses a new logical clock, instead of a physical clock, to annotate the events in order to recover the causal relationship between them. WebTime supports identification of concurrent operations in the resources of a system which makes the resulting framework appropriate for parallel distributed software systems. WebTime is scalable and by definition its instrumentation specification has minimal effect on the performance of a system. 
WebTime has been validated against three different systems including the prototype of a building security system, the simulation of a distributed system and an open source benchmark for bulletin board web applications. 


\section{Acknowledgement}

I am extremely grateful to my thesis supervisor, Professor Roy Gregory Franks, for his support, guidance, useful discussions and more importantly understanding and friendship throughout this research. His deep insight and immense knowledge steered this work to the right direction.

I would like to thank my wife, Dr. Nasrin Hojjat, for her moral support, encouragement and patience, irrespective of her busy life, and my daughter, Sara, for keeping up with a busy dad. This support has been my invaluable asset throughout these years.

I would like to thank all the staffs at the department of Systems and Computer Engineering of Carleton University for keeping everything up and running, one way or another.

I would like to dedicate this thesis to my mother, Zahra, and to the spirit of my father Mohammad-Karam.

Financial assistance provided by the National Science and Engineering Research Canada (NSERC) and Carleton University. 


\section{Table of Contents}

$\begin{array}{ll}\text { Abstract } & \text { iii }\end{array}$

Acknowledgement v v

Table of Contents $\quad$ vi

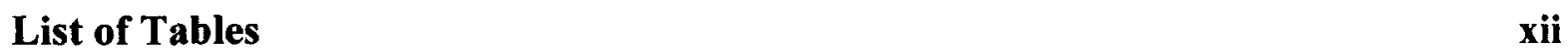

List of Figures $\quad$ xiv

Glossary $\quad$ xviii

$\begin{array}{ll}\text { Chapter 1: Introduction } & 1\end{array}$

1.1 Performance evaluation and methods ............................................................. 2

1.1.1 Measurement................................................................................... 2

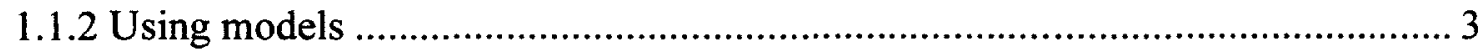

1.1.3 Comparing performance evaluation techniques........................................ 4

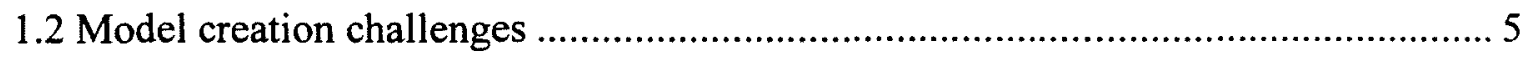

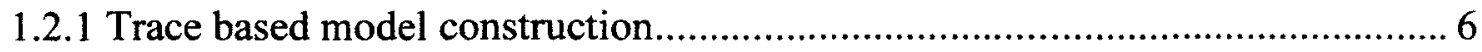

1.2.2 Performance of concurrent distributed systems .......................................... 7

1.2.3 Instrumentation side effects ............................................................ 8

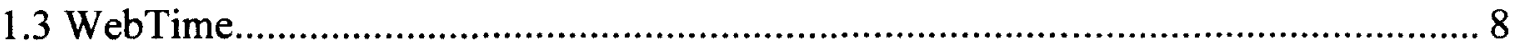

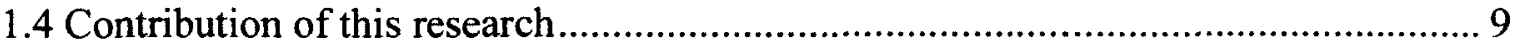

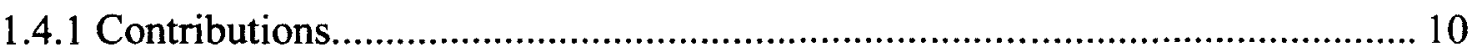

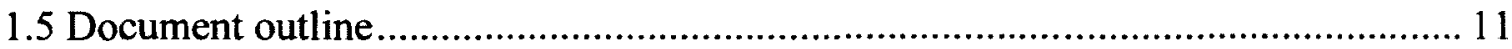

$\begin{array}{ll}\text { Chapter 2: Background } & 12\end{array}$

2.1 Model based performance evaluation - SPE .......................................... 12

2.1.1 Performance integration into software models ................................... 13 
2.1.2 Performance integration using traces ........................................................ 14

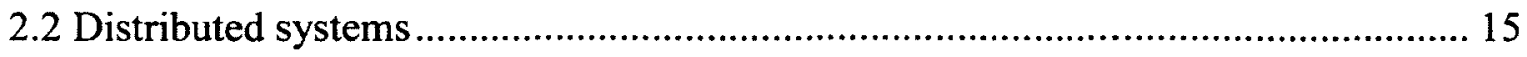

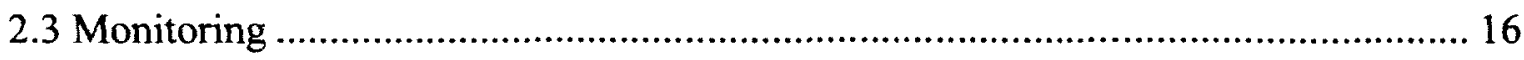

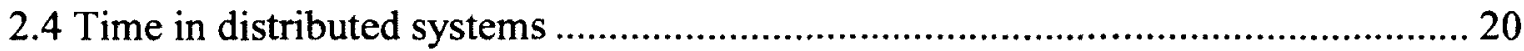

2.4.1 Scalar clock

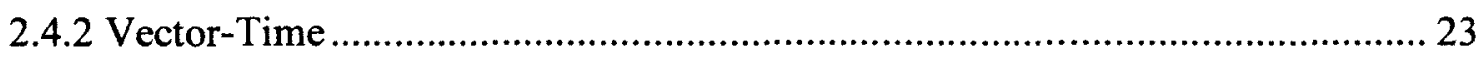

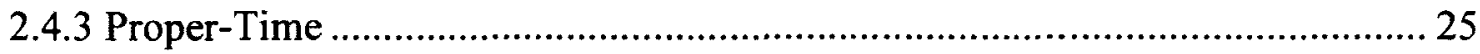

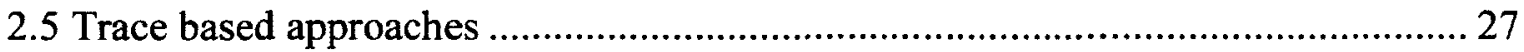

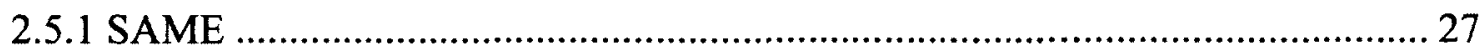

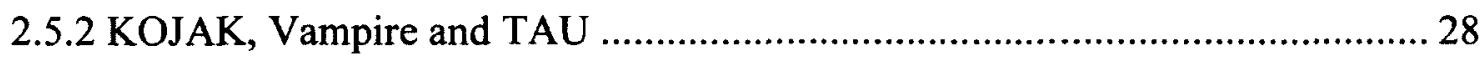

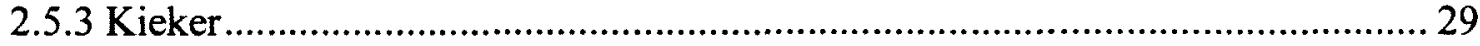

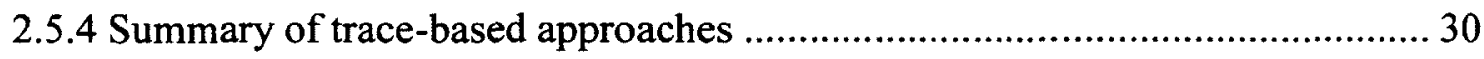

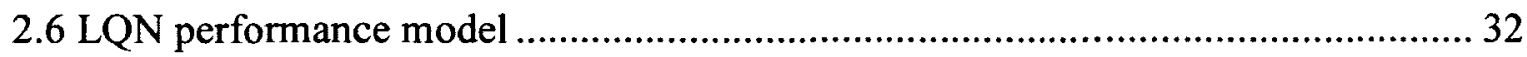

Chapter 3: WebTime, an overview 36

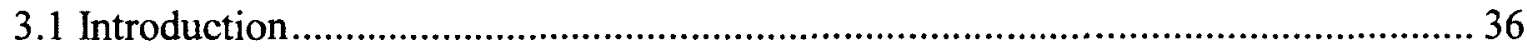

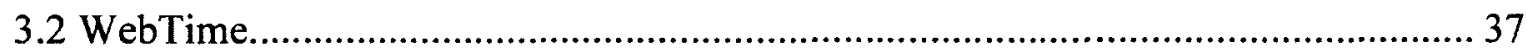

3.3 Model construction process ................................................................................ 38

3.4 Phases and activity graph ................................................................................. 42

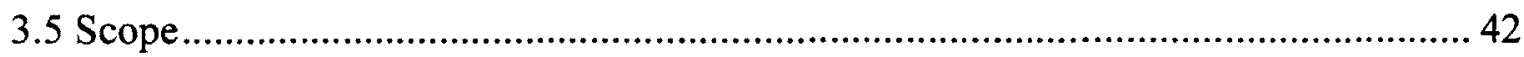

$\begin{array}{lr}\text { Chapter 4: Execution graph } & 44\end{array}$

4.1 Characterization of an event in the execution graph............................................. 44

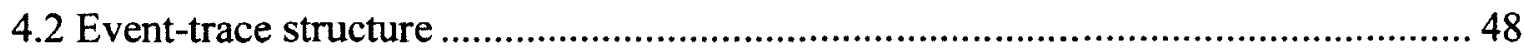

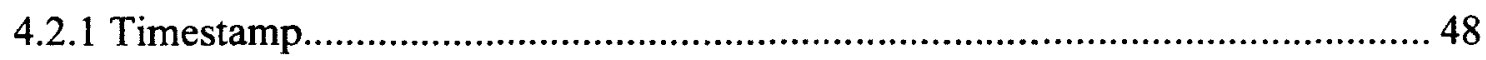

4.2.2 Profile structure ............................................................................................ 50

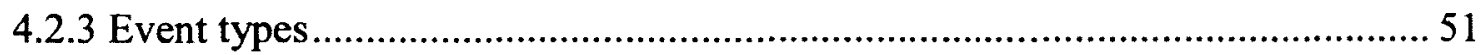

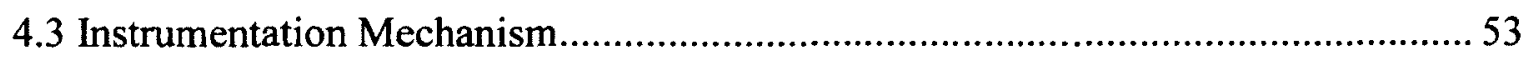

4.4 Event-traces to execution graph transformation .................................................57

4.4.1 Identification algorithm of the internal successor of an event .......................... 57

4.4.2 Identification algorithm of the external successor of an event ........................59 
$\begin{array}{ll}\text { Chapter 5: Event Interaction } & 61\end{array}$

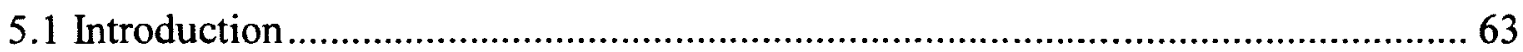

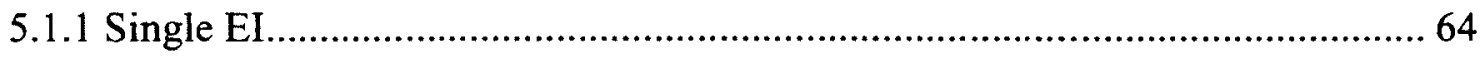

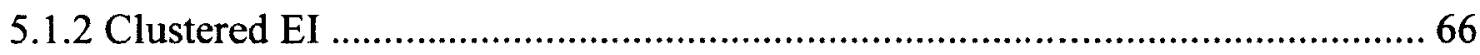

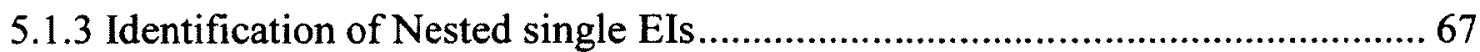

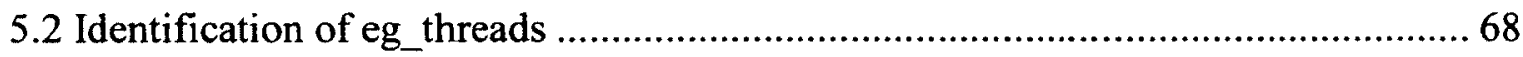

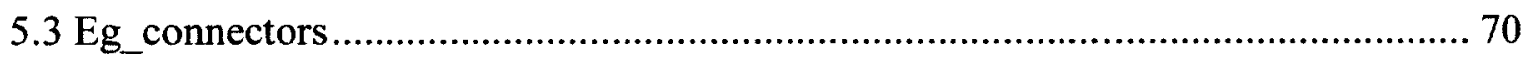

5.3.1 Characterization of an eg_connector .............................................................. 72

5.3.2 Eg_connector identification algorithm .......................................................... 75

5.3.3 Merging of two eg_connectors ......................................................................... 78

5.4 Eg_transitions and segments.............................................................................. 79

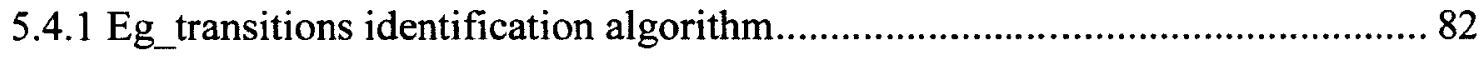

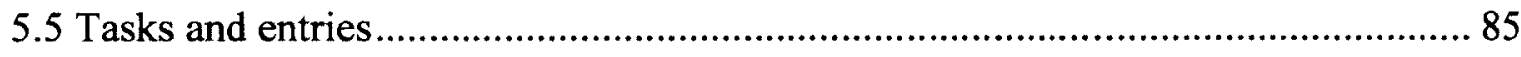

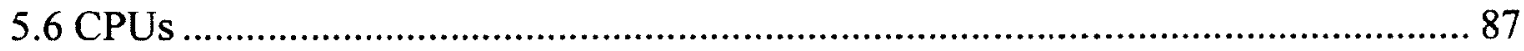

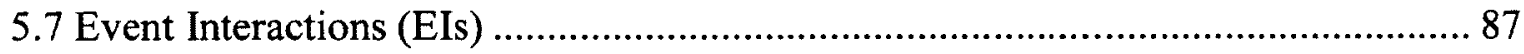

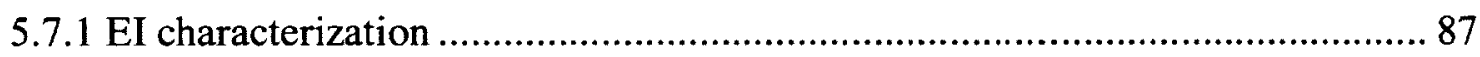

5.7.2 EI-identification algorithm......................................................................... 88

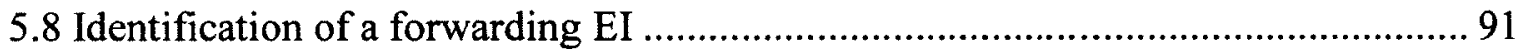

5.9 Identification of the phase of an EI.................................................................. 92

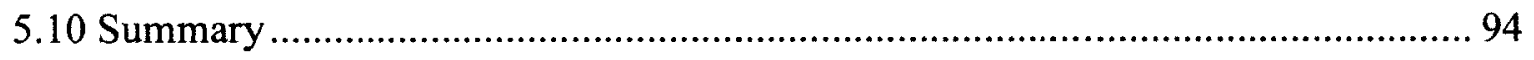

Chapter 6: Tasks Domain - LQN model construction 95

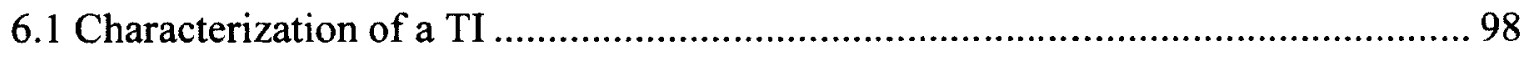

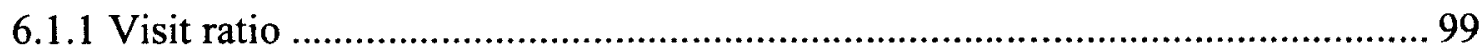

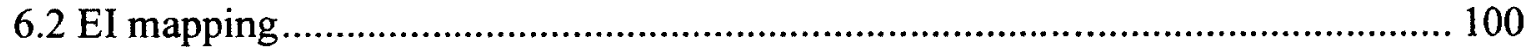

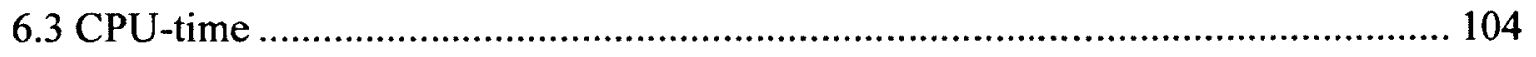

6.3.1 Forming CPU-time vector of an eg_connector ............................................. 104

6.3.2 CPU-time vector of a segment ................................................................... 105

6.3.3 CPU-time vector of an EI ........................................................................... 105

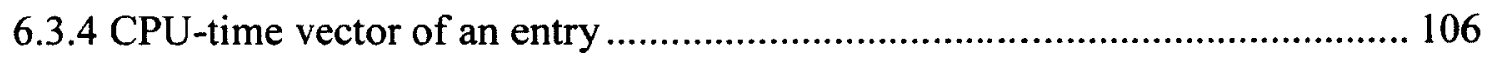

viii 
6.3.5 Determination of the CPU-times of an entry ……......................................... 107

6.4 Computational complexity of WebTime ............................................................ 107

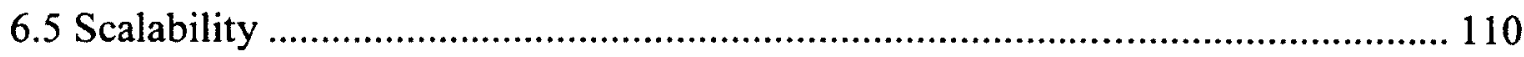

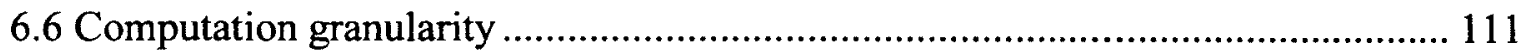

6.7 Constructing a model from sub-models ............................................................. 113

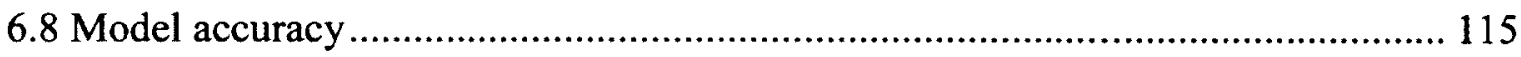

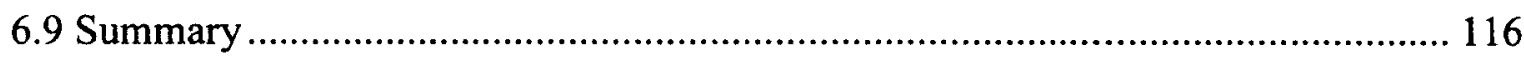

Chapter 7: Construction of an activity graph model 118

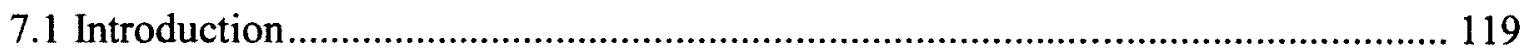

7.2 Activity Graph Model ....................................................................................... 119

7.2.1 Activity graph of a Synchronous interaction ............................................... 121

7.2.2 Activity graph of an asynchronous interaction ............................................ 122

7.2.3 Activity graph of an interaction with second phase ........................................ 122

7.3 Automatic Activity-Graph Construction Process .................................................. 123

7.4 Pre-processing of the Eg_connectors................................................................ 125

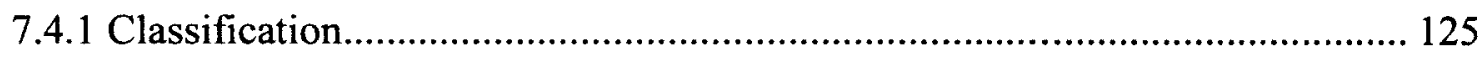

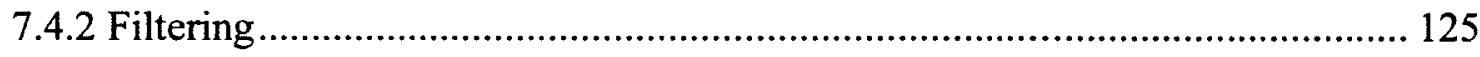

7.4.3 Filtering algorithm of task eg_connectors .................................................. 126

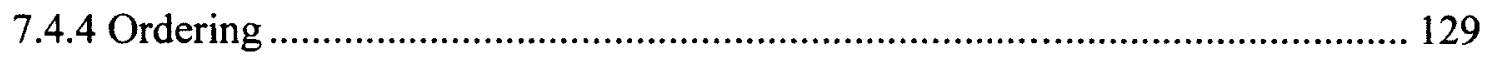

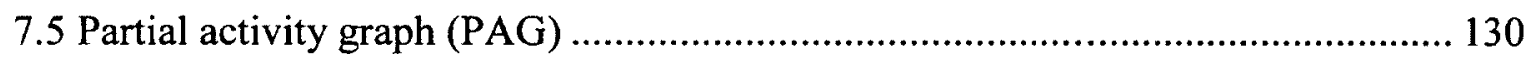

7.5.1 PAG for an eg_connector with end event of type SE .................................... 132

7.5.2 PAG for an eg_connector with end event of type FA..................................... 133

7.5.3 PAG for an eg_connector with end event of type EN .................................. 134

7.5.4 PAG for an eg_connector with end event of type FS .................................... 135

7.5.5 PAG for an eg_connector with end event of type SA..................................... 135

7.5.6 PAG for an eg_connector with head event of type FS................................... 136

7.6 PAG construction algorithm .............................................................................. 137

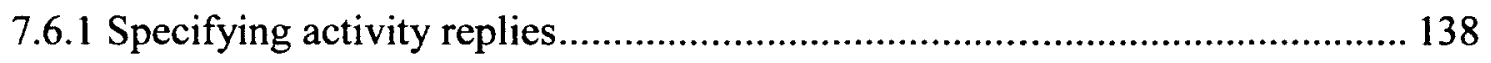

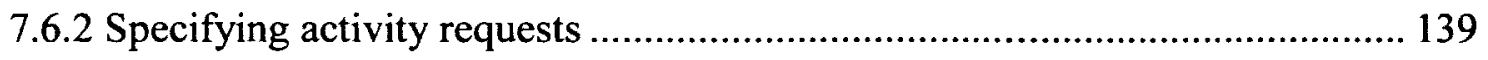

7.7 Activity graph construction.................................................................................. 139 
7.7.1 Specifying the start activity of an entry …................................................ 140

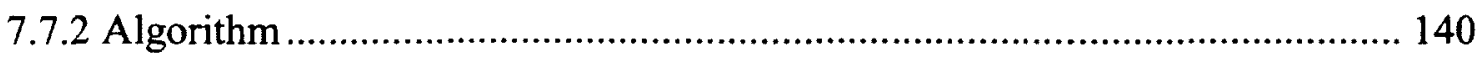

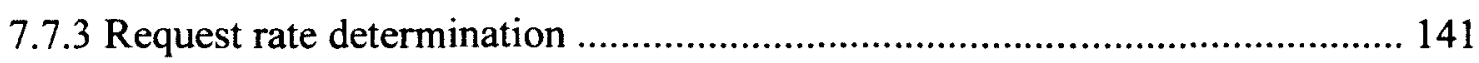

7.8 Example: graph transformer ..................................................................... 142

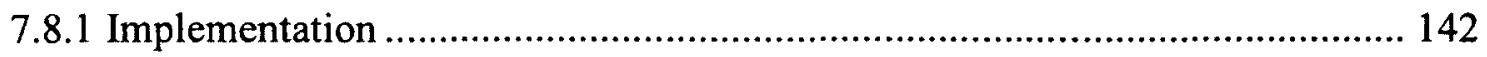

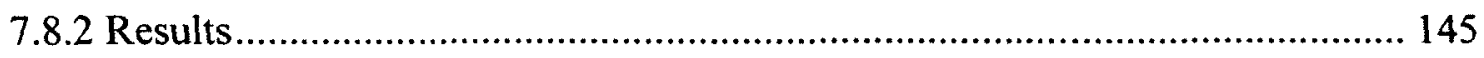

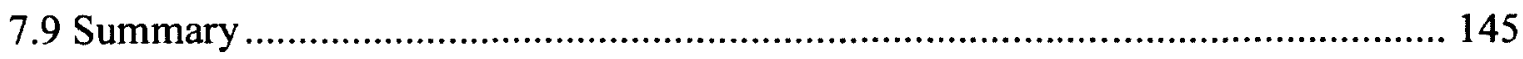

$\begin{array}{lr}\text { Chapter 8: Parasol Case Study } & 147\end{array}$

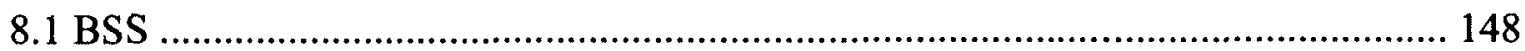

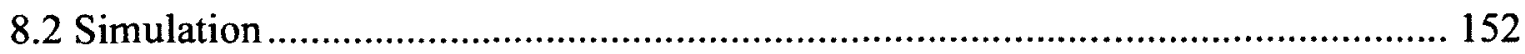

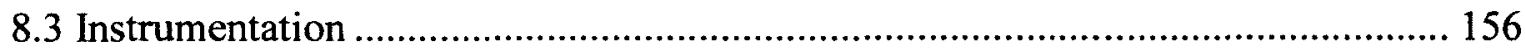

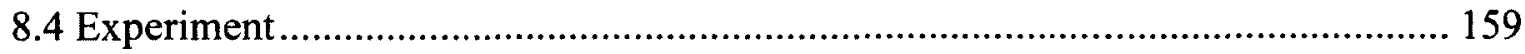

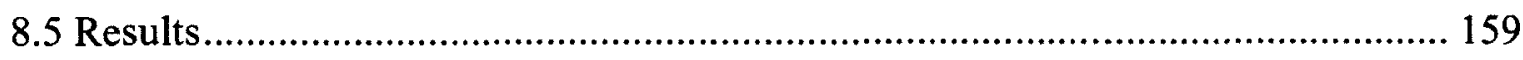

Chapter 9: Java BSS Case Study 162

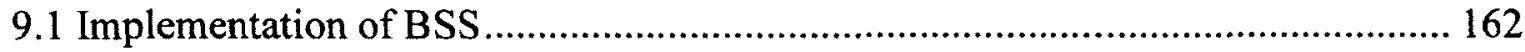

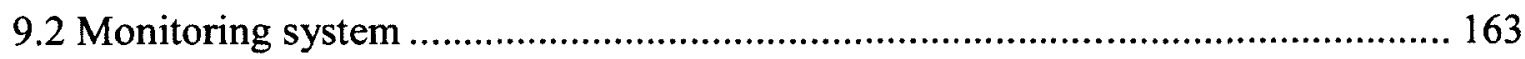

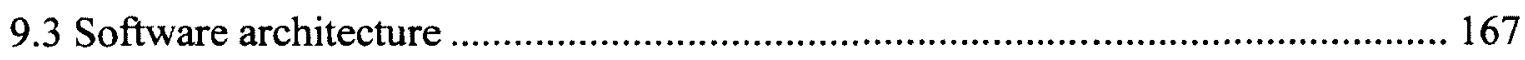

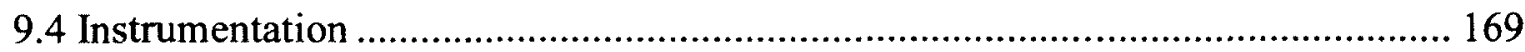

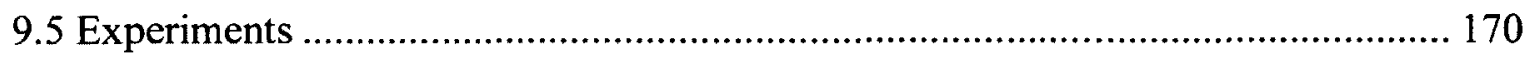

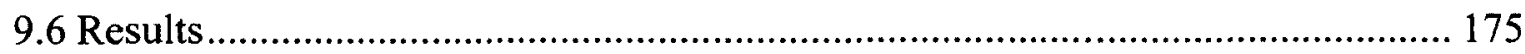

$\begin{array}{ll}\text { Chapter 10: RUBBoS Case study } & 177\end{array}$

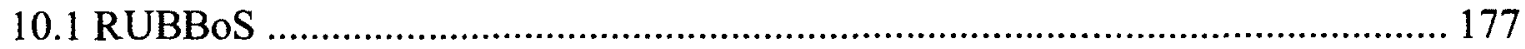

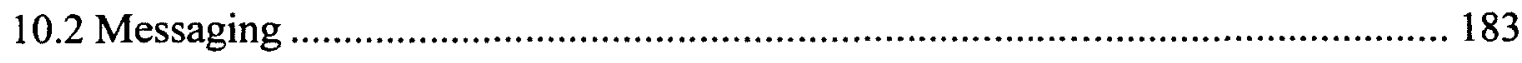

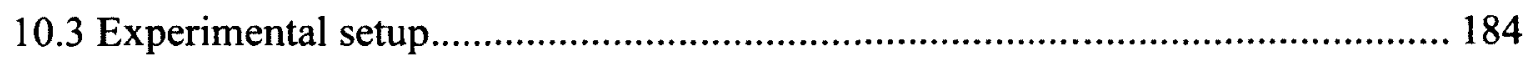

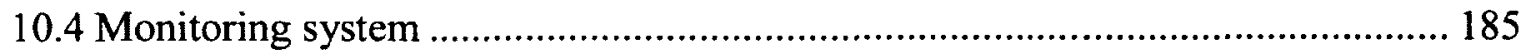

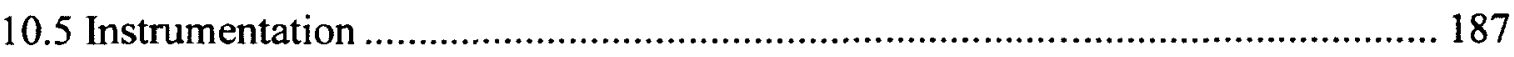

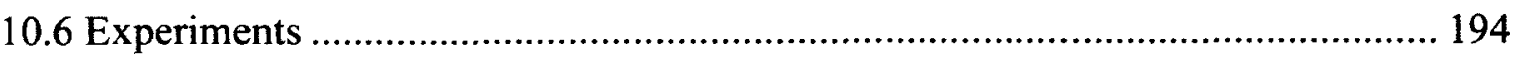

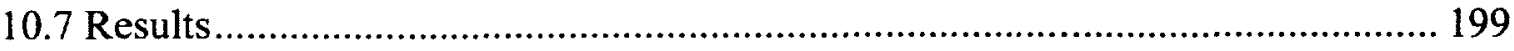


11.1 Contributions of WebTime

203

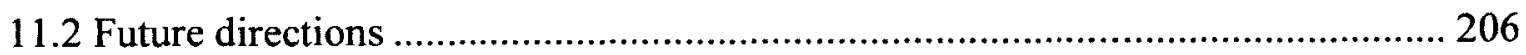

References 


\section{List of Tables}

Table 4-1 attributes of an event in the execution graph...................................................4

Table 4-2 event types and their brief description......................................................... 51

Table 4-3 acceptable event type order between two internally ordered events ................ 58

Table 4-4 acceptable event types between two externally ordered events ........................6 60

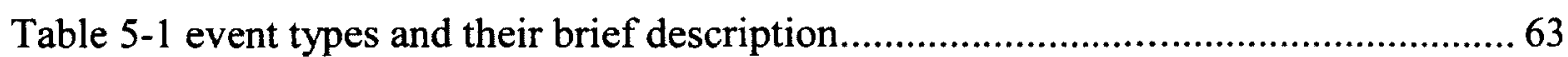

Table 5-2 start-events of the threads in Figure 5-10 ........................................................ 70

Table 5-3 potential event types for head and end events of a connector ........................... 71

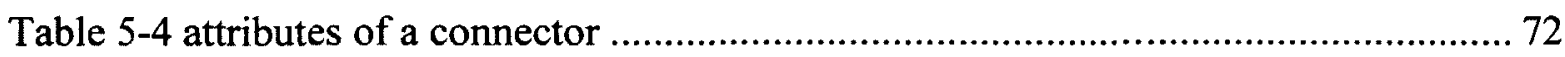

Table 5-5 various eg_connector types in an execution graph .......................................... 75

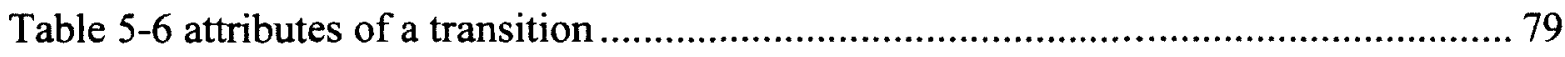

Table 5-7 relation between the type of a transition and its head and end events.............. 80

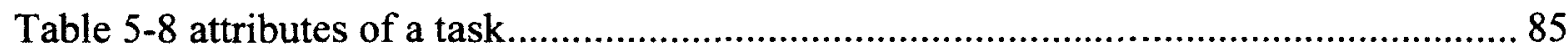

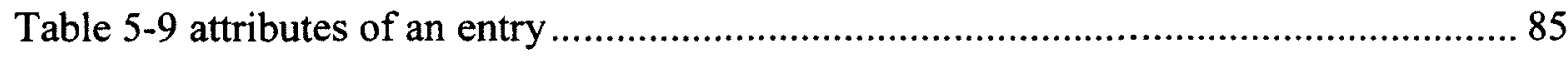

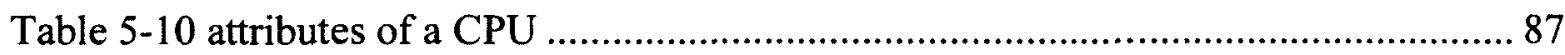

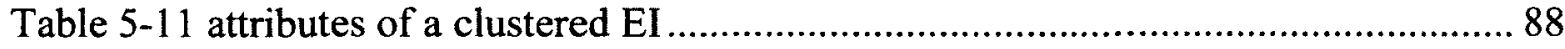

Table 6-1 event types and their brief description.................................................................. 97

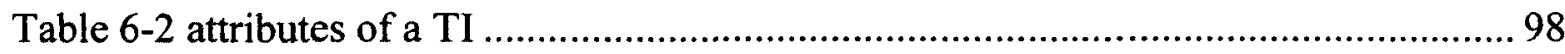

Table 6-3 computation complexity of various processes of WebTime ......................... 110

Table 6-4 attributes of tasks, CPUs, entries and interaction .......................................... 114

Table 7-1 event types and their brief description.......................................................... 118

Table 7-2 the nomenclatures to show activities ........................................................... 120

Table 7-3 the nomenclatures to show the connection between activities ....................... 120

Table 7-4 end interfaces of the PAGs of various connectors........................................ 132

Table 8-1 request rates of the tasks involved in "door access control" .......................... 149

Table 8-2 request rates of the tasks involved in Scenario "video control"..................... 151

xii 
Table 8-3 monitoring functions and the events they monitor ...................................... 158

Table 9-1 Event methods and their correspondence with events types ........................ 168

Table 9-2 software and hardware utilization numbers of BSS tasks and CPUs ............. 173

Table 9-3 total averages of response times obtained with measurement and modeling

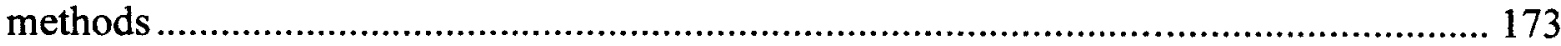

Table 9-4 software and hardware utilization numbers of BSS tasks ........................... 174

Table 9-5 total averages of response times obtained with measurement and modeling

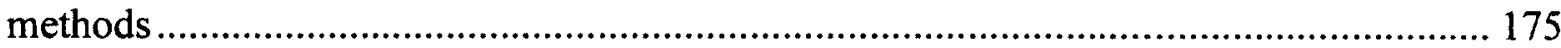

Table 10-1 mapping servlets of RUBBoS to entry identifiers of an LQN model........... 182

Table 10-2 Instrumentation methods and their correspondence with events types ........ 192

Table 10-3 total averages of session periods obtained using measurement and modeling

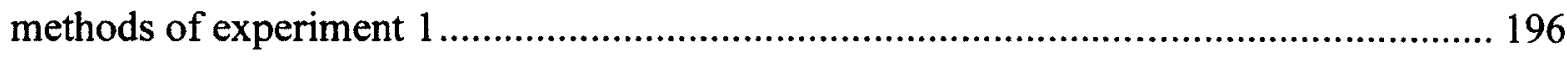

Table 10-4 software and hardware utilization numbers of the RUBBoS resources ....... 197

Table 10-5 user session periods for various number of users ......................................... 199 


\section{List of Figures}

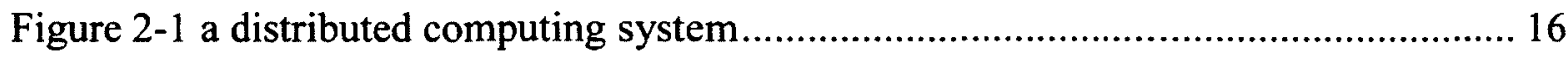

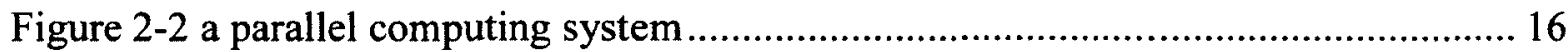

Figure 2-3 layered view of a monitoring system ........................................................ 18

Figure 2-4 a distributed system monitor .................................................................... 19

Figure 2-5 LQN model of a three tier distributed system ............................................. 35

Figure 3-1 automated LQN model building process........................................................ 41

Figure 4-1 execution graph building process.................................................................. 44

Figure 4-2 model of an event in the execution graph ...................................................... 45

Figure 4-3 an execution graph - Task 2 and Task 3 perform second phase operations ... 47

Figure 4-4 a java program to modify the timestamp of an even of type "FS" or "FA"... 54

Figure 4-5 a non-blocking vs. a blocking service request .......................................... 55

Figure 4-6 a reply with a second phase operation vs. a reply with no second phase........ 55

Figure 4-7 index of events following a fork event............................................................ 56

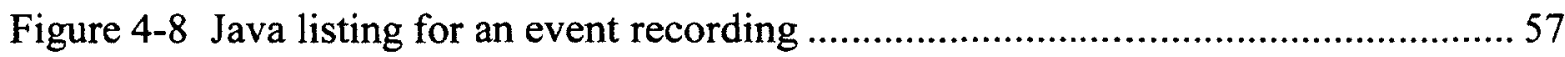

Figure 4-9 algorithm of determining if one event is internal successor of another .......... 58

Figure 4-10 identification of external successor of an event ........................................... 59

Figure 5-1 event interaction identification from execution graph diagram ......................6 62

Figure 5-3 breaking of an execution graph into eg_connectors and eg_transitions ..........6 64

Figure 5-4 forming a segment from an eg_transition and an eg_connector .......................64 64

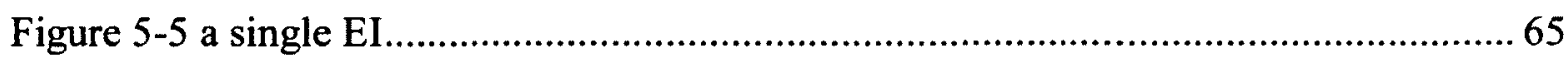

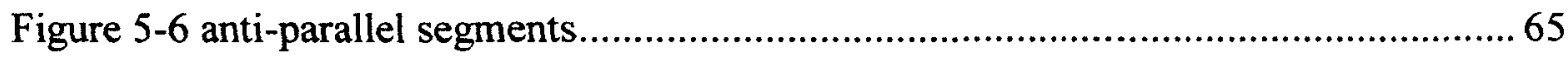

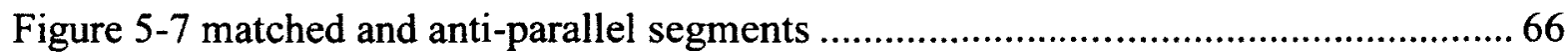

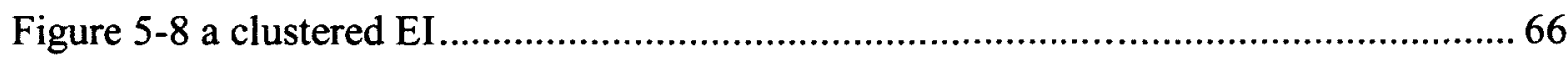

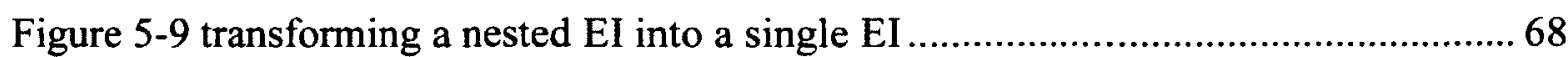

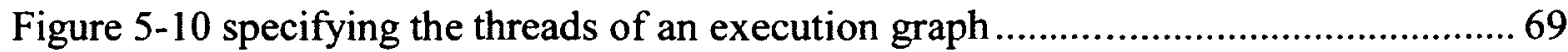


Figure 5-11 algorithm of finding the start-events of an execution graph ........................ 70

Figure 5-12 possible positions of an eg_connector in the execution graph...................... 72

Figure 5-13 transfer of an EI-id to theeg_connectorwhich collapses it ........................... 73

Figure 5-14 naming of connectors in different sections of an EI ................................... 74

Figure 5-15 eg_connector identification algorithm ........................................................ 77

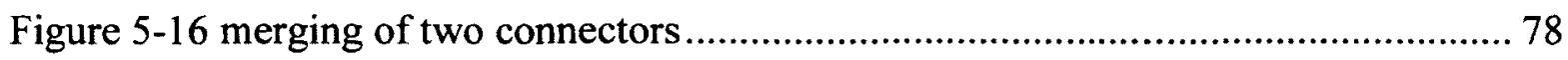

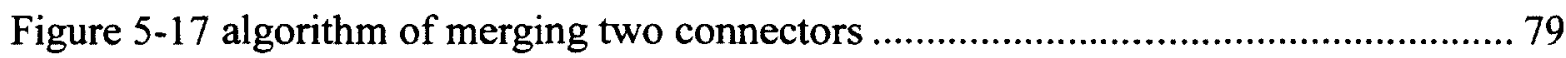

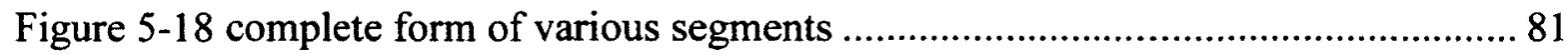

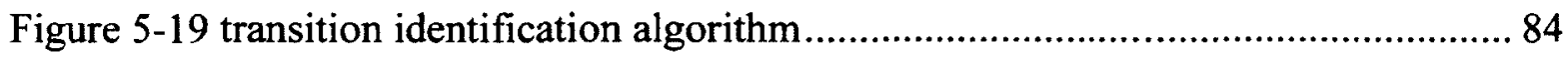

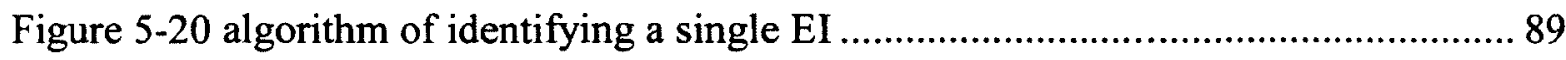

Figure 5-21 algorithm of merging consecutive single EIs to form a clustered EI ............90

Figure 5-22 algorithm of creating the bridging eg_connector of an EI ............................ 90

Figure 5-23 a multi-segment ...................................................................................... 91

Figure 5-24 construction of a forwarding EI from an initiating multi-segment and an

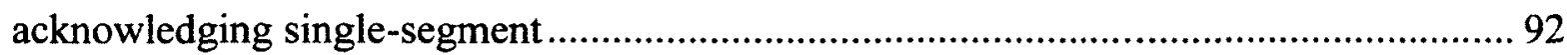

Figure 5-25 event patterns of a first and a second phase Els ......................................... 93

Figure 5-26 finding the phase in which an interaction has occurred .............................. 93

Figure 6-1 construction of an LQN model from CPU, task, entry and EI vectors ........... 95

Figure 6-2 mapping EIs to TIs and entries............................................................... 101

Figure 6-3 mapping of reference, phase 1 and phase 2 EIs to their corresponding TI ... 103

Figure 6-4 algorithm of forming CPU-time vector of connectors of an execution graph

Figure 6-5 mapping CPU-times of two matching segments into their corresponding EI105 Figure 6-6 mapping CPU-times of phase 1 and phase 2 type EIs into the entries of their

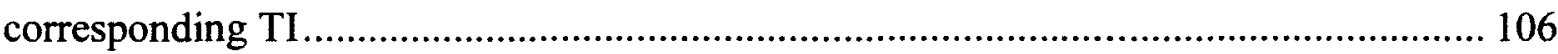

Figure 6-7 pre-processing of task events logs........................................................... 112

Figure 6-8 construction of the final LQN model of sub-models .................................. 113

Figure 6-9 parsing CPUs, tasks and entries data from a sub-model ............................ 117

Figure 7-1 an RPC interaction and its activity graph................................................... 121

Figure 7-2 an asynchronous RPC interaction and its activity graph.............................. 122 
Figure 7-3 an RPC interaction with second phase and its activity graph ...................... 123

Figure $7-4$ the activity graph construction overview diagram ....................................... 124

Figure $7-5$ algorithm of filtering the connectors of a task ........................................... 126

Figure 7-6 a clustered EI between two tasks ............................................................ 127

Figure 7-7 activity graph of the interaction in Figure 7-6 ........................................... 127

Figure 7-8 application of algorithm of filtering connectors of tasks ............................ 128

Figure 7-9 structures of a normal and a bridging connector .......................................... 130

Figure 7-10 the PAG of an eg_connector with SE type end event .............................. 133

Figure 7-11 the PAG of an eg_connector with FA type end event............................... 134

Figure 7-12 the PAG of an eg_connector with EN type end event ............................... 134

Figure 7-13 the PAG of an eg_connector with FS type end event ................................. 135

Figure 7-14 the PAG of an eg_connector with SA type end event................................. 136

Figure 7-15 the PAG of an eg_connector with FS type head event............................... 137

Figure 7-16 algorithm of constructing the PAG of a connector .................................... 138

Figure 7-17 algorithm of construction the activity graph of a task............................... 140

Figure 7-18 execution graph of the test case ........................................................... 142

Figure 7-19 activity graph of the graph transformer example ....................................... 146

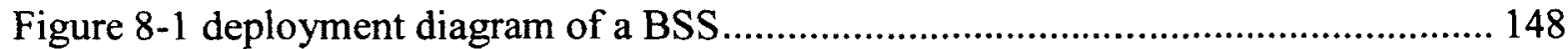

Figure 8-2 Sequence diagram of the door access control scenario ................................ 149

Figure 8-3 Sequence diagram of video control scenario ............................................ 151

Figure 8-4 state diagram of a reference task ................................................................ 153

Figure 8-5 state diagram of a pure server task ......................................................... 154

Figure 8-6 state diagram of an active server task .................................................... 155

Figure 8-7 monitoring sensor locations of the tasks involved in "access control" scenario

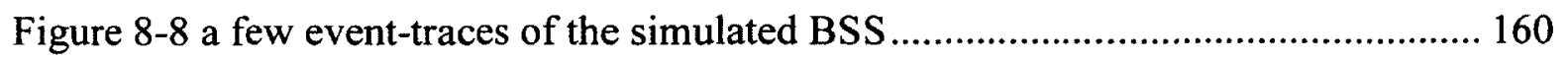

Figure 8-9 the reconstructed LQN model of BSS, created from the base model adopted

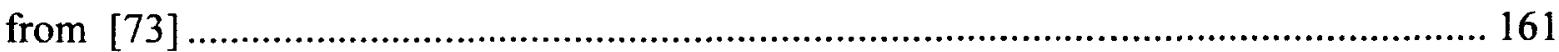

Figure 9-1 deployment diagram of "video control" scenario ......................................... 163

Figure 9-2 logger thread................................................................................................ 164 
Figure 9-3 structure of a manager task implemented in Java ....................................... 165

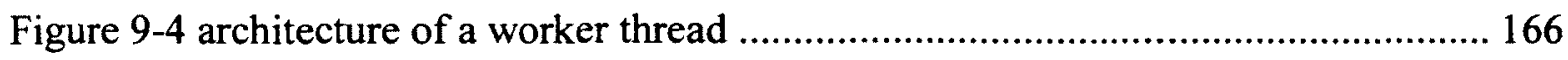

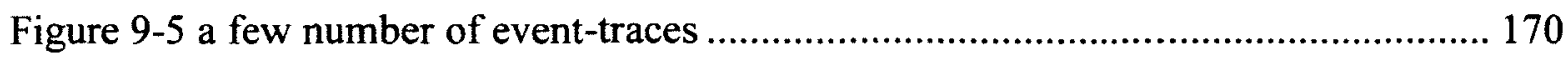

Figure 9-6 distribution of event-traces of tasks to trace files of invocations................. 171

Figure 9-7 the LQN model created for the video control scenario of BSS...................... 172

Figure 9-8 response times to cameras versus the number of cameras being serviced.... 175

Figure 10-1 A three-tier Java Enterprise Edition ...................................................... 178

Figure 10-2 architecture of the emulated web browser ............................................... 180

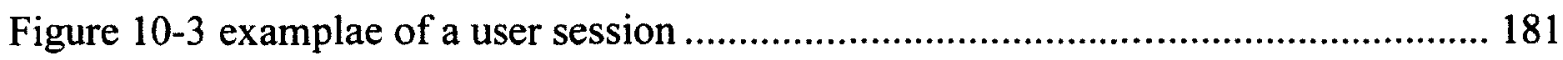

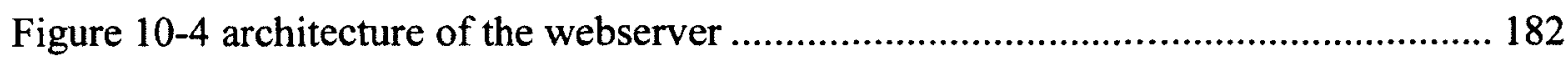

Figure 10-5 deployment diagram of RUBBoS .......................................................... 184

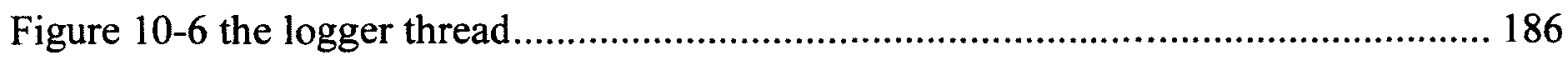

Figure 10-7 the locations in a program where instrumentation methods are added ...... 188

Figure 10-8 the overall LQN model of the first experiment........................................... 195

Figure 10-9 predicted session periods versus numbers of users .................................... 197

Figure 10-10 user session periods versus numbers of users ......................................... 198 


\section{Glossary}

Event An event is a uniquely identifiable runtime instance of an atomic action performed in a non-interleave manner by a single task (page 7).

Task

The LQN formalism models a distributed application as tasks, their concurrency level, queuing mechanism and the interaction between the tasks (page 15, 32).

Entry An entry identifies a particular service offered by a task in an LQN model (page 32).

Wall clock Conventional time (sometimes it is called physical time) (page 20).

time

Distributed A computer network with individual autonomous processors in which systems each processor has its own disjoint local memory. These processors may be physically distributed within some geographical area and their only means of communication is through message passing (page 15)

Parallel Processors in a parallel system interact solely through shared memories systems (page 15).

Concurrent Multi-tasking in each single processor of a distributed application is task defined as concurrency within that processor (page 15). 
Execution

graph

Single-EI

Clustered EI

Task interaction (TI)

Eg_transition

Eg_connector

Segment

Eg_thread

LQN

Phase/
A graph which directly presents the causal or independence relationship between the events of a computation (page 38).

A Single Event Interaction (EI) represents an interaction between a client task and a server task in which the server doesn't make another (nested) interaction with a third task (page 64).

A Clustered Event Interaction (EI) is a set of consecutive single EIs in which the end event of each single EI is the head event of the following single EI (page 66).

When an interaction is specified by the entries of tasks involved in that interaction, it is called a task interaction or TI (page 98).

An eg transition represents a directed arc between two events which belong to two different tasks (page 63).

An eg_connector represents a directed chain of links between the events of one single task (page 63).

A transition and an eg_connector which share one event constitute a segment (page 63).

An eg_thread is a sequence of connected events in an execution graph which can span tasks. It does not necessarily correspond to a real thread of a computation (page 44).

Layered queuing network performance model (page 32).

The service provided by a server task may either occur in phase 1 or 2 . xix 
second phase

The main processing of the server prior to a response is performed in phase one. The server may continue to be busy after it sends an asynchronous reply to the client. This is referred to as the "second phase" of operation which is a common way of performance optimization (page 32, 42).

Activity graph An activity graph is an extended version of a layered queuing network (LQN) model which allows modeling various parallelism techniques employed by software designers to improve the performance of an application (page 42, 118).

Partial activity A partial activity graph is a subset of the final LQN model and is graph (PAG) constructed from an eg_connector (page 130).

Internal

successor

$\&$

External

successor

Visit ratio

Interaction
An event in an execution graph may be preceded by one event and may also be succeeded by one or two other events. The first succeeding event, called the "internal successor", represents an event in the same eg_thread of this event. The second succeeding event, called the "external successor", represents an event in a different eg_thread (page 44).

The visit ratio is the ratio of the number of calls to the destination entry over the number of calls to the source entry (Page 95).

A general term for a request and reply between two tasks. In a distributed system, this is often implemented using a remote procedure call. 
An activity is the basic unit of behaviour in a layered queuing network. Activities are linked together by a directed graph to represent the precedence, or causal relationship between events (page 118). 


\section{Chapter 1: Introduction}

Performance of a computer system is defined as the response time and throughput of that system. It is an essential quality attribute of computing systems which is affected by everything from the low level hardware all the way up to the application software. Lack of performance manifests itself as poor responsiveness, which is an unacceptable delay in the responses received to service requests, and as a result, prevents a system from meeting its objective performance expectations. From a business point of view, ignorance of the performance requirements in the development cycle of a software system results in lost income due to unplanned tuning cycles, reduced competitiveness, damaged customer relations and even abandonment of the product [1]. As the field of computer systems advances, the computer industry is becoming more competitive and it is now more important than ever to ensure that the alternatives selected provide the best costperformance trade-off. As such service providers and designers of computers systems are all interested in performance evaluation since their goal is to provide the highest performance at the lowest cost. A computer system that has unjustified delays in the response to a service request, unnecessarily wastes human time, increases cost and squanders the principle resources of an organization.

The sections that follow describe briefly the challenges and the common methodologies used for performance evaluation and introduce the solution provided by this research and its contributions. 


\subsection{Performance evaluation and methods}

Performance evaluation is an art. Like a work of art, successful evaluation cannot be produced mechanically. Defining the real problem and converting it to a form in which established tools and techniques can be used and where time and other constraints can be met is a major part of analysts' art.

\section{The art of computer systems performance analysis, Raj Jain [2]}

In order to meet the performance requirements of a computing system, software developers should be able to assess and understand the effects of various design decisions on the performance of the resulting system throughout its design cycle. This is particularly important at the early stages of the development cycle, where changes to the design can be made easily and effectively. In other words the performance evaluation should be integrated into the development cycle of a software system to allow for a performance oriented design, which is the goal of the software performance engineering (SPE) [3]. Adoption of SPE approach ensures that a high performance product will be created at the end of the development cycle.

To evaluate the performance of a computing system at each stage, a designer has essentially two options: measurement and modeling.

\subsubsection{Measurement}

Regardless of common belief, measurement alone may not give accurate results simply because many of the environmental parameters, such as system configuration, type of workload and time of measurement, may be unique to the experiment and may not represent the range of variables found in a real situation. Measurement can be 
performed when a working version of the software under development is available. Identification and fixing the performance problems by using only measurement is costly since it might require rearchitecting the design and the measurement, multiple times, in order to obtain an optimum design. Measurement provides a software designer with data associated with one concrete structure. The data generated from this condition has limited flexibility to allow one to effectively locate the source of design problems. For example it would be challenging to isolate from the data, the application behaviour and the system parameters [2]. This uncertainty would prevent one from extrapolating the results to make an expert judgment on how to fix potential problems.

\subsubsection{Using models}

Modeling is the other way forward to evaluate the performance of a system. In this method the system under test is modeled by a simulation system or by mathematical expression, such as a queuing network (QN) [4], which is then used as a surrogate for the real system. In general, modeling requires many simplifications and assumptions that can modify the accuracy of the end result. Nevertheless modeling techniques are usually extensively tested and their results are validated against the measurement to make sure their predictions of the performance behaviour are often in an acceptable range.

Unlike measurement, models can be built and used at the earliest stages of the development cycle, even when a system has not been constructed. By considering that performance failures are most often due to fundamental architectural or design deficiencies [3], modeling can be an effective means to identify fundamental design pitfalls. Models are flexible for varying environmental conditions, design alternatives, 
and system sizes. The alternatives to an architectural design, or even the performance requirement set for a design, can be assessed by comparing those requirements with the performance evaluated by models.

\subsubsection{Comparing performance evaluation techniques}

The selection of the right performance evaluation technique depends upon the time and resources available to improve the performance of a system and the desired level of accuracy. The goal of every performance study is either to compare different alternatives or to find the optimal parameter values. Models, due to their ease of use and modification, generally provide the best insight into the effect of various parameters and different alternatives with the least amount of time and resources. Measurement is the least desirable technique in this respect. It is not easy to tell if an improved performance is a result of some random changes in environment or due to particular parameter settings.

Scalability of the results is probably the key justification when considering the expense and labor of measurement. Performance models are very scalable with minimal effort and cost but most people don't trust the results from the analytical models because of a lack of understanding of the technique [2]. In fact the results from all the simulation and analytical techniques which are applied to create a modeling formalism undergo extensive validation against actual measurement.

The modeling approach has undeniable benefits especially when combined with the measurement approach for evaluation of the performance of a system. For example the measurement techniques, being susceptible to experimental errors and bugs, can be 
validated using modeling. Likewise measurement can also be used to verify and validate the results of the modeling. Alternatively, the modeling technique can be used to find the appropriate range of system parameters for a required performance level. A measurement technique can then be used to study the performance in a range which reduces the number of measurement cycles and that can then use resources in a more productive manner.

\subsection{Model creation challenges}

Regardless of the attractive features of performance models creation of them is not a trivial task. In a manual way, an analyst must obtain an accurate structural view of the software system by reviewing the software documents or even using its source code. The information, such as the system's behaviour, the components involved and the interactions among the components should be captured and incorporated into the resulting models. This large amount of information is unwieldy even for a moderately sized system and requires people who are highly skilled in this field which will add to the time and cost of the performance model creation process. Because of likelihood of human mistakes, improper handling of this information may also reduce the representativeness of the resulting model which will result in a lack of prediction accuracy. If the model creation process is used in a SPE approach, the required efforts are multiplied further by the number of required iterations. Overall the cost, time and expertise required to make the modeling approach an attractive way of performance evaluation may offset its benefits and if this situation is not properly managed the whole approach might be rendered not trustable, useless or very costly. 
To make the model creation process more attractive an alternative method should be used. This approach automatically generates the model from a software application itself. This way the end result would be more accurate, less dependent on special expertise, less prone to human mistakes, much less time consuming and less costly overall. Because of its automatic feature, this approach will also leverage an analyst's efforts to quickly build performance models by introducing enough complexity to increase the accuracy of the models and to making it representative of the real systems she/he models.

\subsubsection{Trace based model construction}

One way to obtain the initial information from which a model can be constructed is to trace and record the execution of a running computing system then convert, in an automated fashion, the resulting trace log into a model. At each instant during the run, the internal operation of this system can be viewed as a number of customer objects requesting services from server objects. This approach should be able to capture the interactions between the objects of the system and determine the demands that customer objects make on the other objects of the system, such as the average required CPU processing time and the number of visits on each object to complete their requests. The end result would be a high-level descriptive architecture of the system, and the parameters which describe the expected demands on each object of the system.

A trace based performance model can be constructed throughout and in the early life cycle of a software system product. This is due to the fact that modern software engineering promotes releases that are often built in an incremental fashion so that the most important functionality can be delivered as early as possible $[5][5][5][6][7]$. The 
initial releases of an incremental approach allow for creation of early trace based performance models of this system.

There are a variety of methods to extract the sequence of information, or path of execution, such as instrumentation by virtual machines, operating systems or using profilers such as "Quantify" [7]. This research has created methods to manually instrument the source code of a system and suggests extensions of this approach towards automating the instrumentation process.

\subsubsection{Performance of concurrent distributed systems}

Distributed systems are powerful computing systems that combine the processing power and memory capacity of multiple heterogeneous computing systems which are often geographically dispersed and might belong to different organizations. These systems have attracted tremendous attention because they can solve intensive computing problems within a specified time frame or under real time condition with processing power that is available using simple inexpensive computing systems. The pre-dominant programming model for the distributed system is message passing which may be combined with concurrent program execution within each single computer. Achieving satisfactory application performance in these systems is dependent upon the ability of these systems to evenly distribute the processing load among their constituents [8][9]. This is a very difficult task and it requires the system to deal with a variety of problems such as the heterogeneity of the computing systems, a hierarchy of varying bandwidths and latencies in internal networks [10]. The estimation of the performance of these 
systems would provide a great help to manage uncertainty, overcome their load balancing problem and utilize these systems to their best of capacities.

The key issue in distributed systems for collecting trace data for model building is that the local time of the computers in these systems are not necessarily synchronized. In order to correctly trace the execution of events, it is necessary to have the notion of a global monitor for all the computers in these systems in order to record the traces of events in the order in which they have been executed. An event is a uniquely identifiable runtime instance of an atomic action performed in a non-interleave manner by a single task (the task is defined in Section 2.6).

\subsubsection{Instrumentation side effects}

Adding instrumentation to a software system should have minimal effect on its operation and performance. This is especially important for a trace based approach to minimize the incurred execution cycles and communication overheads due to the instrumentation of software probes and tracing.

\subsection{WebTime}

This research presents a framework of specifications and methodologies, called WebTime, for automatically constructing the performance model of a distributed application based on the hierarchical client-server paradigm. WebTime is a trace based approach and presents tools and methodologies for instrumentation, monitoring and constructing a layered queuing network (LQN) [11] performance model of a distributed application. WebTime introduces a new structure for a timestamp, which is independent 
from wall clock time, and performs a post-mortem analysis of the events log of the application to construct its LQN model.

Several monitoring methods have been introduced in the literature such as ProperTime [12][13] and Vector-Time [14][15]. These methods, which are explained in the following chapter, generally suffer from an inability to capture concurrent operations in a computing system and lack of scalability for larger systems.

\subsection{Contribution of this research}

To facilitate the high performance software development process, this thesis has advanced the state of the art for automatic performance model creation methodologies by improving upon event tracing, software instrumentation and transformation methods and techniques. This thesis introduces a new structure for the timestamps attached to the events of a distributed system. A performance extraction framework has been constructed which consists of methodologies and techniques to determine the software probes needed to be added by an instrumentation system and its corresponding implementation mechanism. It also includes the creation of tools to perform the transformations to identify the software architecture and behaviour to create an LQN model. The resultant framework is fully automated, practical and generates results with an acceptable accuracy level.

This method in its entirety is validated in several ways including instrumentation of RUBBoS [16], which is an open source benchmark for bulletin board web application, and construction of its performance model. The prediction of response times, 
throughputs, and utilization from the model are compared with those provided by RUBBoS.

\subsubsection{Contributions}

In summary the contributions of this research are listed below:

1. This research has provided a set of specifications and methodologies for automatically constructing the performance evaluation model of a message passing distributed software system, end to end, from its execution.

2. This research has introduced a new structure of a timestamp which is attached to the messages of a distributed system for tracing its events. This structure imposes minimal communication and processing overhead, compared to available methods, and helps to effectively identify the architecture of the distributed system.

3. This research has implemented methods to determine the service times and the service request rates of the resources of a distributed computing system

4. This research has introduced sensor codes (probes) needed to instrument the events of a distributed application by attaching the monitoring timestamps to the events of that system

5. This research has introduced a formal methodology for transformation of the events $\log$ of a distributed system to the final LQN performance evaluation model of that system.

6. This research has been applied on an open-source webserver application, RUBBoS, and demonstrated satisfactory results 


\subsection{Document outline}

This dissertation is organized as follows: Chapter 2 reviews the current state of performance evaluation techniques of software systems. Chapter 3 provides an overview of WebTime framework. Chapter 4 describes an execution graph which is the result of the first step transformation of the events $\log$ of an application and introduces the structure of an event-trace and also the instrumentation specification of WebTime. Chapter 5 describes the methods used for identification of interactions between the events of a distributed system. Chapter 6 illustrates the process of mapping the identified event interactions to the interactions between the tasks of a computation system and determining the service request rate for each task and finally construct the LQN performance model of that system. Chapter 7 introduces a methodology to construct the "activity graph" which is a more detailed form of an LQN model. Chapter 8 applies WebTime on a simulated version of a distributed application to generate its events log and construct its LQN model. Chapter 9 applies WebTime on the prototype of a distributed application which is created using socket technology of Java and discusses the results. Chapter 10 applies WebTime on an open source web application and analyzes the results. Chapter 11 summarises the current work and introduces the future directions for this research. 


\section{Chapter 2: Background}

This chapter describes the research associated with the construction of a performance model of a distributed application through monitoring logical clock and model construction by traces. This chapter also describes the layered queuing model (LQN) which is the model used by this work. The most prominent research about each of these topics will be briefly explained.

\subsection{Model based performance evaluation - SPE}

Software Performance Engineering (SPE) is a prominent method for integration of performance into the development cycle and defining it as a milestone.

SPE is a method for constructing software systems which meet performance goals [3]. It includes the techniques for gathering data, constructing performance models, evaluating design alternatives and finally verifying the models and validating the results. In each step of model construction, a performance evaluation helps making decisions for tuning the design based on the identified potential problems, instead of postponing the fix until the implementation phase.

The main component of SPE methodology is the estimation of performance which is repeatedly performed in the development cycle. SPE doesn't provide or suggest any specific method for creation of models, thus the designers are still required to put 
considerable effort to understand the techniques needed to create suitable performance models which are related to, but not the same as, those commonly used in designing of software systems. An attractive solution to help a designer would be both hiding of the modeling techniques and presenting the details of statistical results in a meaningful way to the software engineers by automating this process [17].

Integration of performance into software development practices is an ongoing research target. Performance engineers tend to use performance specific scenarios in which the system behaviour is described by execution graphs. Software engineers use conventional software scenario descriptive notations such as UML use cases [18], collaboration and sequence diagrams. Obviously integration of these two fields, in one way or another, would benefit the software development practices by ensuring that the aspects of high performance software are respected in the software design.

\subsubsection{Performance integration into software models}

One way to achieve full SPE capability is to add annotations into models of software such as the standard "UML Profile for Schedulability, Performance and Time" (SPT) [19] and its replacement, the "UML Profile for Modeling and Analysis of Real-Time and Embedded Systems" (MARTE) [20]. A variety of different forms of integrating performance into software models have been introduced. Petriu and Woodside [21] used Use Case Maps (UCM) [22] to extract an LQN performance model of a software system. UCM is a visual representation of the requirements of a system, using a precisely defined set of symbols for responsibilities, system components, and sequences. UCMs are created with a free software tool called Use Case Maps Navigator (jUCMNav) [23]. This tool 
generates as output an XML listing of the system requirements which is transformed to an LQN model to evaluate the system's performance.

Kähkipuro [24] has introduced a method which allows automatic extraction of relevant performance metrics of a distributed object oriented software from extended UML diagrams and presents model results back into the UML diagram. The resulting UML diagram presents both the functional and performance features of the software. This method transforms those features of UML which are significant for the system performance into a textual notation, named the "performance modeling language" (PLM), from which, in a later step, an extended QN model of the software is created. The QN model is then solved with the method of decomposition [25] and the result is brought back to the UML diagram.

From UML into Petri Net [26] is another approach to extract performance models from UML. This method uses activity diagram as the main source of "do activity" part of state chart and takes a formal approach to translating it to a Stochastic Petri Nets model (SPT) by which the performance metrics of a software program is extracted.

\subsubsection{Performance integration using traces}

A different approach for incorporating performance evaluation in the development cycle is though tracing the execution of a software system and constructing its performance model from the trace. In this approach a software system is instrumented to generate an events $\log$. This events $\log$ is then analyzed to construct a performance model. The biggest benefit of this approach is its capability to extract a model from the dynamic behaviour of a system. However it requires the availability of the subject 
software system. By considering that the modern software engineering practices promotes incremental releases [5][6][7], tracing is an appropriate solution for automation and integration of performance model construction in the development cycle. Tracebased approaches are revisited in Sections 2.4 and 2.5.

\subsection{Distributed systems}

A distributed system, in a general sense, is a computer network with individual autonomous processors, each of which has its own local memory. These entities may be physically distributed within some geographical area. A distributed system may have a common goal, such as solving a large computational problem. The purpose of a distributed system is to share its resources by coordinating their use and providing communication services to the users. A resource is an abstraction of the range of things that can be usefully shared in a networked computer system such as processors, disk, printers, files and databases. The resources of a distributed application are called tasks in an LQN model. A brief characterization of the aspects of a distributed system which are important to it performance analysis is provided below.

A distributed system is characterized using the logical or functional distribution of its processing capabilities [27]. This system contains more than one processor which each should have independent threads of control. Processors communicate with one another using only messages, which take a finite time to travel from one processor to another. Processors in a parallel system interact solely through shared memories. The interaction through messages is the main distinction between a distributed and a parallel system. 
Finally, since resources are not shared in a distributed system, the processors don't have a global clock time. [28]

A distinction here is made between the concurrency and parallelism terminologies. In this document the term "concurrency" is used to refer to processing in a distributed system.

\subsection{Monitoring}

Event of a computing system are collected using a monitoring system. A general description of a monitoring systems is provided here which is mainly extracted from [2].

A monitor is a process or set of possibly distributed processes whose function is the dynamic gathering, analyzing, presenting, interpreting, and acting on information concerning an application as that application executes. The actions can include the changing of configuration or the controlling of a system's parameters and states. These systems can therefore provide increased robustness, security, fault-tolerance, and adaptability.

The main difference between the various implementations of monitoring systems is their overhead. Overhead is defined as the consumption of system resources, such as CPU or storage, by the monitoring system. For example the data recorded by a monitoring system may be recorded in a storage system. Software monitors tend to have higher overheads and lower event execution rates than hardware ones. However they have higher recording capacities than hardware monitors. Software monitors are suitable for the situations where the rate in which events are generated is low to limit their overhead. 
Monitors have sensors in various components of a system. A sensor is an entity which senses occurrence of an event. An event is a change in the component which a sensor monitors including a process context switch, beginning of seek on a disk or arrival of a packet. Upon being triggered, a sensor generates an event-trace. When a sensor is triggered by a change to the entity, the sensor will be invoked to trace the entity. Tracing is performed synchronously with the change in the value of the entity meaning that the tracing is triggered by the change. When the value of the object changes, the sensor reports the new value to the monitoring system or immediately executes a particular code. Depending upon the mechanism which triggers the monitor into action, a monitor can be classified as event driven or a sampling monitor. If a sensor is triggered by a request from the monitoring system it is called a sampling monitor. Sampling is an on-demand tracing by a monitoring program. Tracing as the result of a sampling is asynchronous with a change in an entity's value. When a monitoring routine decides to collect an entity's value, it sends a message to the appropriate sensor, and the sensor returns the current value. The sampling monitors are activated at fixed time intervals by interrupting sensor systems.

The result displaying ability is another way by which monitors are classified. On-line monitors display the system state either continuously or at frequent intervals. Alternatively, batch monitors collect data to produce a log which can be analyzed by a separate analysis program.

In a general sense the various functions of a monitor are divided into a number of layers, as shown in Figure 2-3. 


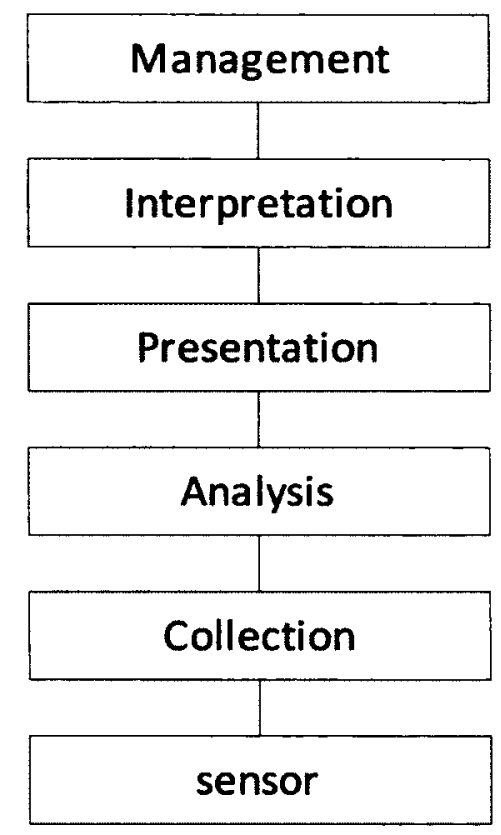

Figure 2-3 layered view of a monitoring system

- The sensor layer gathers raw data on individual components of the system.

- The collection layer collects data from various sensors.

- The analysis layer analyzes the data gathered at various collectors collectively.

- The presentation layer deals with human user interface and produces reports, displays and alarms.

- The interpretation layer, which could be a human being or an expert system, makes meaningful interpretation of data. 
- The management layer makes decision to set or change system parameters or configurations based on interpretation.

A monitor may contain zero or more components from each of these layers and there is a many to many relationship between the successive layers, as shown in Figure 2-4.

Generally there is one sensor element for each component but the elements of other layers have more limited numbers and may be located on remote servers. For example there may be one analyzer for a number of collectors where all the collectors will send their events logs to that analyzer node.

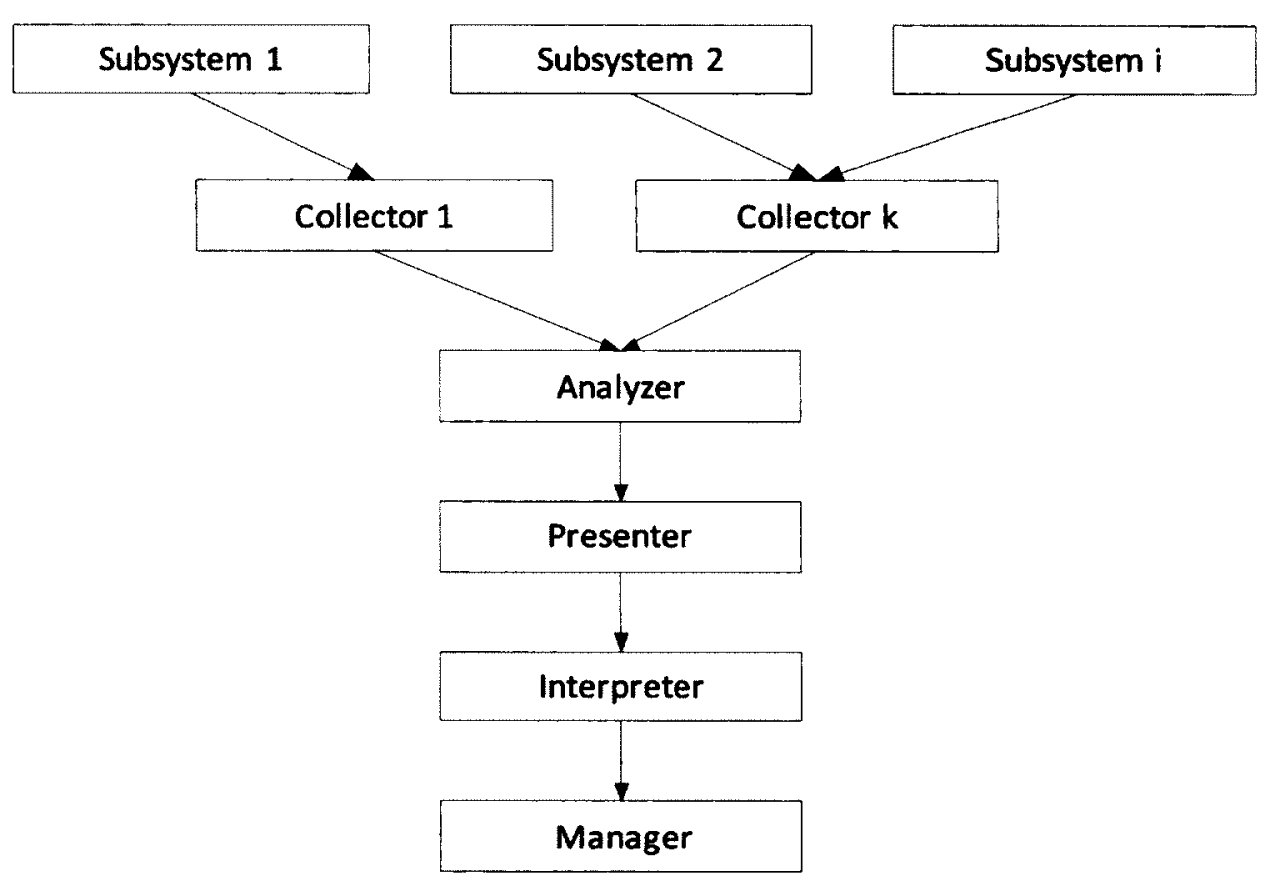

Figure 2-4 a distributed system monitor 


\subsection{Time in distributed systems}

In order to model a distributed application it is necessary to reconstruct the precedence relations and the interactions between the events of this system by studying the causal, or a cause and effect, relationship between them. Except for shared memory systems, generally, it is not possible to use the conventional time, which is called here the "wall clock time", for capturing causality between the events of a distributed system because of the following reasons:

- Processes often don't have access to a global clock, therefore they are not synchronized.

- The messages' delays are variable and nondeterministic.

There have been many studies $[29][30][15][14]$ to define a new notion of time to overcome these problems. This notion of time, called the "logical clock", could work as a partial substitute for the physical time of a distributed application if it has the following important characteristics as necessary conditions:

- Allow the inference of the potential causalities between events,

- exclusion of a causal relation if a future event seems to influence a past event.

As another requirement, a logical clock should be easily realizable, without using the physical clock. These points can be explained by visualizing an abstract model of a distributed system in which processes communicate with messages and execute their internal events sequentially. These events execute at specific instants in time and are limited to send, receive and internal events. Internal events occurring at a particular process are linearly ordered by their local sequence of occurrence, and each receive event 
has a corresponding send event that happened earlier. Formally, the causality relation, '<', can be defined as a transitive relation on the set of events such that for any two events $e, e^{\prime}$ :

1. If $e$ and $e$ ' happen at the same process, e is the immediate predecessor of $e^{\prime}$, or

2. If $e^{\prime}$ is the receipt of a message, $e$ is the send event associated with that message.

In other words the time always moves forward and a future event cannot influence a past event. This relation, which is named as "happened before" by Lamport [29], is the heart of any sensible logical clock. It can be noted that, with respect to the causality relationship, the exact global time at which an event has happened is of no concern when the two conditions mentioned above holds.

In the execution of a distributed application nothing happens between successive events. Hence the time needs to be advanced by occurrences of events and is therefore discrete. There should be a function $C: E \rightarrow T$ which assigns a timestamp $C(e)$ of a suitable "time domain" $T$ to each event $e$ of the event set $E$. The comparison of timestamps of different events should allow drawing certain conclusions about the relation of the events. It seems to be plausible that at least the notions "earlier" or "later" should exist within any logical clock. Hence a logical clock should be a (possibly partial) ordered set, $(\mathrm{T},<)$. A reasonable requirement on $C$ is that it conforms to the causality relation:

$$
e<e^{\prime} \rightarrow C(e)<C\left(e^{\prime}\right)
$$

As a consequence of the above condition, which is often referred to as the "clock condition", the following properties hold: 
1. For each process, time is monotonically increasing,

2. the logical clock of a send event is always earlier than the logical clock of the corresponding receive event.

\subsubsection{Scalar clock}

An implementation scheme of the logical clock was presented by Lamport [29] which is based on an integer domain, $T$, for the timestamp values. It is realized by a system of counters $C i$, one for each process, and a simple message handling protocol:

1. When executing an internal event or a send event at process $P i$, clock $C i$ "ticks": $C i=C i+1$.

2. Each message contains a timestamp that equals the time of the send event.

3. When executing a receive event at $P i$ where a message with timestamp $t$ is received, the clock is advanced: $C i=\max (C i, t)+1$.

The timestamp $C(e)$ of an event is defined to be the value of the local clock just after it is being updated when executing the event.

As it stands, the scheme allows different events (in different processes) to have the same timestamp; events that are causally independent get assigned timestamp values as if they happen in a certain order. This is so because mapping the partially ordered events onto linearly ordered integers causes some information about the structure of the application be lost. Hence, scalar clocks lack a desirable property of time: by checking the timestamps of events, it is usually not possible to assert that "some events could not affect some other events". This defect is resolved with another implementation of a timestamp called "Vector-Time". 


\subsubsection{Vector-Time}

An aspect of the wall clock time, by assuming that it is ideally synchronized when used in a distributed system, is that all the processes can have access to it. The "VectorTime" method, introduced independently by Mattern [15] and Fidge [14], follows the same idea to develop a global logical clock which is accessible by all processes. This global logical clock is a vector of integer numbers in which each component represents the current scalar clock of each and every process involved in a distributed system. However because of variable message propagation delays, a process cannot have access to the instantaneous logical clock of other processes. This issue is mitigated by having each process compute an optimal approximation of this notion of global time. Here each process $P_{i}$ is equipped with the timestamp $C_{i}$ which is a vector of length $n$, where $n$ is the total number of processes involved in the system. The $j^{\text {th }}$ element of this vector represents the scalar clock associated with process $P_{j}$. Each process must be informed at the earliest possible moment about all known events which did already happen. The mechanism is explained below:

The $i^{\text {th }}$ element of this vector ticks immediately before the event $e$ of process $P_{i}$ is executed:

$$
C i[i]=C i[i]+1
$$

Each message is timestamped with the current value of the sender's vector clock. When receiving a timestamped message, a process combines its own time knowledge $\mathrm{Ci}$ with the timestamp $t$ it receives by performing: 


$$
C_{i}=\sup \left(C_{i}, t\right)
$$

Where "sup" denotes the component wise maximum operation. In order to be able to compare time vectors $u$ and $v$, the following relations for ' $<$ ', ' $\leq$ ', ' $\|$ ' are defined as follows:

$$
\begin{gathered}
u \leq v \text { iff } \forall i: u[i] \leq v[i] \\
u<v \text { iff } u \leq v \& u \neq v \\
u \| v \text { iff } \neg(u \leq v) \& \neg(u<v)
\end{gathered}
$$

Where $\leq$ (and also ' $<$ ') is a partial order. Relation ' $\|$ ', which is reflexive and symmetric (but non-transitive), can be viewed as a generalization of the concurrency of real time. The main property of vector clocks is that by checking the timestamp values of events, their causal relationship can be reconstructed, as shown below:

$$
\forall e, e^{\prime} \epsilon E: e<e^{\prime} \text { iff } C(e)<C\left(e^{\prime}\right) \text { and } e \| e^{\prime} \text { iff } C(e) \| C\left(e^{\prime}\right)
$$

This property has attracted attention of a large number of researchers for characterizing and troubleshooting distributed applications using Vector-Time method $[31][32][33][34][35][36][37][38]$. However, in its full generality, the Vector-Time method may be impractically expensive for long-lived computations because of the size of timestamp, which is as large as the number of process instances created. [14]. Large number of processes increases the size of the timestamp and it, in turn, increases the processing and communication overhead of this approach. It is also the main obstacle to 
the scalability of this method. There have been several methods to circumvent this problem. To reduce the communication overhead one can send only the elements of the timestamp which are updated with respect to the previous sent to a receiver. This approach requires maintaining, via two further auxiliary vectors, the value of the local counter, when a vector was last sent to each other processes, and the last updated elements of the timestamp [39]. There also have been many methods for using constant size timestamp in exchange for less accuracy [40][41]. The difficulty and strength of identifying the patterns of events involved in an interaction are the other side of the problems associated with Vector-Time approach. Baldoni and Ranyal [42] describe identification of patterns of events interaction as dealing with vectors of vectors which is of increasing difficulty. In its original form, Vector-Time is unable to correctly reconstruct an interaction when a message associated with a different interaction overtakes the massage sent from the first interaction. However, a modification of this approach has fixed this problem at the cost of higher computation and local memory consumption load [43].

\subsubsection{Proper-Time}

A different implementation of the logical time is presented in Proper-time [12][13] . This method annotates the events of a computation by a fixed number of parameters, as listed below, to reconstruct the computation.

1. Task name

2. Task event index

3. Service period index 
4. Operation name

5. Operation thread index

6. Thread event index

7. Event type

The task index of an event is created by a counter in the task in which the event occurs (the task is defined in Section 2.6). The sequence of events created this way is named the "task event graph" or TEG. The operation index is an integer number which is traced through the sequence of executing events. When it is received by an event, either through a message or by simple shared memory parameter passing, it will be incremented as the application runs. The graph created this way is named the "operation event graph" or OEG. To reconstruct a computation, this method analyses these two graphs and combines them into a new graph, which is named "task and operation event graph" or TOEG. This graph then is used to decide whether an internal transition or an interaction has been occurred. By definition these graphs are created separately and independently. Analyzing TOEGs of a computation should allow identification of various interaction types including synchronous, asynchronous, or forwarding.

In practice, in a distributed computation in which tasks have concurrent operations, finding an association between various TEGs, resulted from various threads, is very challenging. This operation is necessary in order to identify the interactions performed in that computation. Despite the presumption of independence between TEGs and OEGs, the method uses OEGs to associate different TEGs belonging to the same process, which contradicts their stated independence. More specifically, this method introduces the 
"service period index" parameter in a TEG and traces it through its associated OEG. This has made the event interaction identification process in this method very difficult, expensive and even inaccurate, considering that this tracing has to be done all the way through all the nested interactions.

\subsection{Trace based approaches}

In this section a number of trace based approaches are described. Proper-Time and Vector-Time are also in the category of trace based approaches but since they also have introduced new logical clock structures, they were described in the previous section.

\subsubsection{SAME}

SAME [44] is a method which uses a trace-based approach to retrieve architecture of a computing system in order to create its performance model. This approach uses the wall clock times at which the methods of this system are called and returned to identify the interactions between the components of this system.. This method is able to identify various types of interaction including synchronous, asynchronous and forwarding as long as the nodes don't have internal concurrency. The identified nodes and interactions, along with user supplied information, are used to create the LQN performance model of this system. SAME assumes that a global monitor exists which records events in the order of occurrence. This assumption is the key obstacle for applying this approach on a distributed application. 


\subsubsection{KOJAK, Vampire and TAU}

KOJAK [45][46] uses a comprehensive instrumentation scheme to create events log which will be used by an analyzer. The analyzer provides a high level view of the raw events $\log$ and generates an analysis report, which is used to visually present the system activities. The instrumentation of the user supplied code will be performed based on its nature. For example KOJAK instruments OpenMP (open multi-platform) [47] constructs by redirection of OpenMP library calls to instrumented wrapper functions on the sourcecode level. It uses TAU [48][49], which is described below, or a compiler supplied profiling interface to instrument user functions. All these schemes call a run-time library, which provides mechanisms for buffering and events log creation. By running the executable created from the instrumented program, the events log will be generated.

Vampire [50] is a set of sophisticated trace analysis and visualization tools which uses the monitoring scheme of KOJAK and TAU to create event-traces which are then used to visualize the detailed run-time behaviour of parallel OpenMP and MPI (message passing interface) [51] software programs. It uses a wall clock time for its timing requirements and exchanges clock synchronization information at the very beginning and the very end of a trace run. During the tracing period every process records all local events according to the local asynchronous time. In a post-processing step, the timestamps are translated to a global time [46], i.e. one local time selected as the master. Differences in offset and speed between the local times and the master are interpolated linearly between the initial and final synchronization point. 
TAU is a flexible framework of tools to measure and analyze the performance of deployed parallel systems. It defines an abstract computation model that captures general architecture and software execution features that can be mapped to various complex system types. TAU's instrumentation mechanisms support several types of events, including events defined by code location, library interface events, system events, arbitrary user-defined events and events associated with message passing and multithreading parallel execution. The instrumentation layer interfaces with the measurement layer through a measurement API. The performance measurement part supports two measurement forms of profiling and tracing. TAU uses the local wall clock and provides an interface for choice of timer to support different timing sources. The TAU data analysis and presentation utilities are open; they offer text-based and graphical tools to visualize the performance data as well as bridges to third-party software, such as Vampir [50], for sophisticated trace analysis and visualization.

\subsubsection{Kieker}

Kieker [52] is an extensible framework for software instrumentation, logging, analysis and visualization. It employs a non-intrusive instrumentation technique using aspect-oriented programming (AOP) [53] utilizing frameworks such as AspectJ [54], Spring AOP [55] . Kieker uses a common data structure for monitoring records in all the components that produce or consume monitoring data, with the support for distributed request tracing. For analysis of monitoring data, Kieker provides several visualizations of a system's runtime behaviour, such as UML sequence diagrams, dependency graphs, and Markov chains. These models are extracted from recorded application-internal traces 
originating from system provided services. The analysis may be performed online or offline.

\subsubsection{Summary of trace-based approaches}

Trace-based approaches can be classified into three categories:

1. Wall-clock timestamp (SAME)

2. Wall-clock timestamp with tracing (Kieker, TAU)

3. Logical timestamp (Proper-time, Vector time)

The method in the first category, SAME, uses wall-clock times at which the methods of a software application are called and returned to construct the interaction tree of this application. This method is not appropriate for distributed applications due to an asynchrony between the wall-clock times of the processors of a distributed application. This limitation is mitigated in the methods of the second category. These methods, generally, add some logical annotations such as event count and interaction depth to the events of a software application [52] to compensate for the time difference between the processors of a distributed application. These methods suffer from extra processing overhead due to the complexity introduced by this mixed timestamp structure. The methods of both of the first and the second categories also suffer from handling and processing of high volume events logs since the wall-clock timestamps are required to be high resolution numbers.

The methods in the third category don't use any element of the wall-clock time in their timestamp structure. Therefore, their events logs are lighter, they don't need to process high resolution numbers and they are appropriate for distributed applications. 
However both Proper-time and Vector-time suffer from unnecessary complications which have offset these benefits. Vector-time is not scalable and suffers from high message communication and events log processing overhead for computations which require large number of processes. Proper-time suffers from an inaccuracy due to its unnecessarily complex model to describe the relationship between events.

There are other aspects by which these methods can be compared. None of these methods automatically determine the service times and request rates of tasks from an events log. Except Vector-time, none of these methods support task concurrency in a distributed system. Proper-time fails in this respect due to the inaccuracy which is introduced by its events model complexity. Finally, none of these time-based techniques are able to identify special patterns of interaction between tasks which are introduced in distributed applications to increase efficiency. Examples of these interactions are asynchronous and early reply or second phase interactions [56]. In the former case a client task doesn't block for a service reply but continuous its other operations before receiving a reply. In the latter case, a server task sends a service reply to a client as soon as it is ready, regardless of whether or not the whole operation associated with that service request is finished. It performs the remainder of the operation after sending the service reply, which is called the second phase of operation. Proper-time supports asynchronous interactions. However, none of these methods support the second phase interaction. 


\subsection{LQN performance model}

There are several formalisms to study the structure of a computing system and identify bottlenecks such as Petri Nets [57], Stochastic Process Algebras [58], and Queuing Networks [4]. WebTime uses the LQN [59][60][61] model to study the performance of a distributed application because of the close match of the model with that of a distributed software system. The LQN formalism models a distributed application as tasks, their concurrency level, queuing mechanism and the interaction between the tasks. In an LQN model the interaction between two tasks may canonically extend to other subsequent interactions between other tasks, which it is assumed that they are located at different layers of services. The layered structure of the LQN extensively simplifies the modeling of an application as compared with formalisms such as QN in which there is a flat relationship between the tasks of an application.

In an LQN model a task can act both as a client, to request a service, and as a server, to provide services to the other tasks. Lower layer servers offer services to clients at higher layers. The service times of the higher level tasks will accumulate with those of lower layer tasks because of blocking calls. Distributed systems may also suffer from a software bottleneck which is caused either by a software server waiting for the replies from a lower level server or its internal processing load. LQN captures these issues by calculating the service time of a process for its internal execution, its requests to other servers and the queuing delays it experiences during those requests. LQN can also be used to study many other issues such as the impact of different algorithms, load balancing by using various concurrency levels, the effect of varying users, and for sensitivity 
analysis, for example, to determine the limit of multi-threading performance gains before an overhead (extra memory requirements for the heap or cache hit) kicks in [60].

A task consists of one or more "entries" by which the task accepts service request messages. An entry identifies a particular service offered by a task and its parameters. A database server, for example, may be represented by a process with two entries: one that corresponds to a query operation and the other for an update operation. The behaviour of a task in two different environments may also be represented by two entries which have different parameters. For instance, when the task represents the services offered in a node of a distributed system, such as a WAN, it may exhibit more delays, thus its interactions will be represented with a different entry than when the task represents that of a shared memory bus node.

A task is characterized by a queue for the service requests it receives, its level of concurrency and one or more entries. Entries have directed arcs to other entries to represent requests for services. An entry may either block, until it receives a reply, or continue operation. The former case is referred to as a synchronous request or remote procedure call (RPC) and the latter as an asynchronous interaction (the former case is also sometimes referred to as blocking and the latter as non-blocking interaction). The receiving entry may forward the request to an entry in another task and that entry, in turn, to another entry in a different task and so forth until the request in ultimately replied to the originating entry. This later style of interaction between tasks is called a forwarding interaction. An entry may continue to be busy after it sends an asynchronous reply to an initiating interaction. This is referred to as the "second phase" of operation which is a 
common way of performance optimization (for example, for transaction cleanup logging and delayed write operation). Tasks execute on the "processors" which are often CPUs in the system being studied.

An entry is characterized by its number of phases, the type of interactions it makes with the entries of other tasks, and its "service times" and "request rates". The "request rates" specifies the mean number of service requests the entry makes to the entries of the tasks in a lower layer. The "service time" specifies the mean total required CPU-time for the entry in units of time. Each phase has its own individual service time and request rate $[56]$.

Figure 2-5 shows an LQN model in which "task A" makes synchronous interactions with "task B" which, in turn, "task B" makes a different synchronous interaction with "task C". In this figure tasks are represented by parallelograms containing parallelograms for their entries. The arcs between entries represent messages or requests to a responding task. "task B" is multi-threaded and this is indicated in the figure by the stack of parallelograms. The maximum number of the concurrent threads in "task B" is shown in the curly brackets. "task A" is a pure customer task or the "reference task" which is a surrogate for workload generator of the system. The ovals represent the processor on which the tasks are executed. The numbers in square brackets are service times and the numbers in the parenthesis are request rates of interactions for their two operation phases. Multiplicities are shown by a number in the braces. A complete description of the LQN model is provided in [56]. 


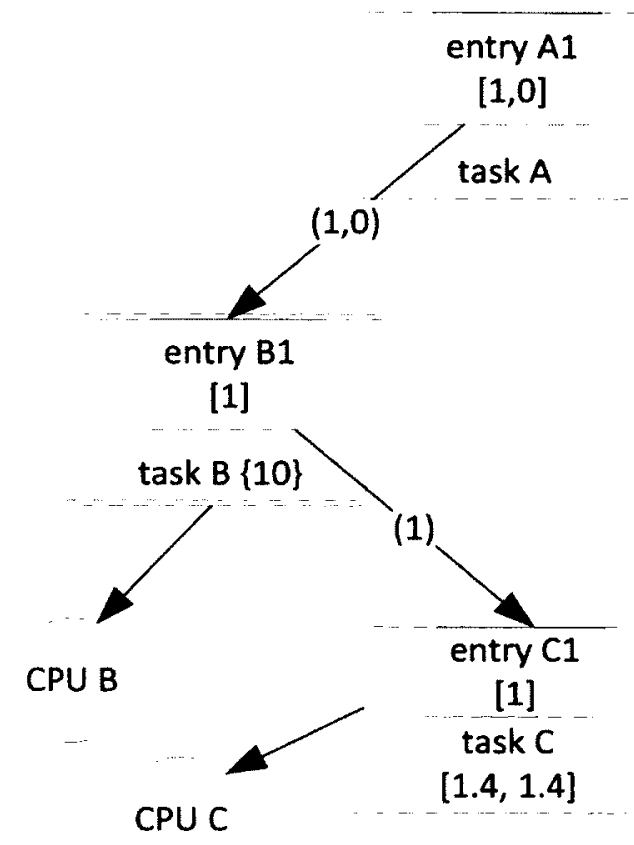

Figure 2-5 LQN model of a three tier distributed system 


\section{Chapter 3: WebTime, an overview}

This chapter provides an overview of WebTime. WebTime uses the execution traces of an application as its input to construct the Layered Queuing Network (LQN) model of that application. The LQN model is suitable to model a distributed application.

\subsection{Introduction}

WebTime can be used to model the performance behaviours of a distributed application. To solve a problem in a distributed system, the problem is divided into many parts, each of which is assigned to a particular task. Tasks are software components whose execution are scheduled and may be performed concurrently on different nodes.

The main cause of delays, and thus insufficient performance, in a distributed application is contention or waiting of tasks for services. The delay mechanism of a distributed application is explained here in a simple fashion. A distributed application is modeled by tasks and the interaction between them in order to request services or to reply to the requests the tasks receive. A task can either play the role of a server to reply to requests, or the role of a client to request for services of other tasks. The requests received by a task may be queued when the task is heavily loaded or the requesting tasks may contend for the offered services. Either way the result is the blocking of a requesting task until the service is granted. The accumulated service time, contention time or the 
queuing delay of the target task will be perceived as delays and lack of performance of the requesting task. These delays will eventually lower the service quality of the whole software application. The capability of server tasks to perform concurrent operations may reduce the service time of the upper layer tasks and increase the overall performance of the computing system if the tasks have enough resources to handle all the requests. A software performance bottleneck will emerge if a task doesn't have enough concurrency for the requests it receives. A hardware bottleneck will emerge if a node's CPU is fully utilized.

\subsection{WebTime}

WebTime traces the events of a distributed system in order to capture the dynamic behaviours of a concurrent computation. A model is constructed by logging the events of an operation and performing a series of post-mortem analysis steps on the events log. The usual way of tracing events is to attach a timestamp to the events and tracking them. Tracing of the events of a concurrent operation on a single-CPU computer or even a tightly coupled multi-CPU system is easy since all the timestamps are created with respect to a single reference which is the system's clock time. In a distributed system there is not a time reference for all the dispersed nodes by which the events can be timestamped. WebTime used a virtual clock concept for creating the timestamp of events.

WebTime uses an event-trace structure which consists of the "timestamp" and some local and static specifications of an event which is called "profile". The profile of an event must consist of the identifiers of the node and the task in which the event occurred as well as some specifications such as the multiplicity of the CPU of the node and the 
concurrency level of the task. The timestamp, which is in fact a new logical clock, specifies the position of an event in a computation such as the thread in which the event occurred and the position of the event in that thread.

WebTime is a scalable approach. It creates a partial model, called a sub-model, for each single invocation of a system. It then combines all the sub-models to construct the final LQN model of the system. Therefore, a large computation can be broken down into many small invocations which are individually processed. Eventually the results are combined to create a model for the whole computation.

\subsection{Model construction process}

WebTime constructs the LQN performance model of a system in a fully automated fashion. The model construction process consists of:

- identification of tasks,

- identification of interactions between the tasks,

- determination of the required CPU-time for performing a service in a task, and

- determination of the number of requests a task makes to each of the other tasks to service a request of another client task.

The first two points above refer to the "model architecture" and the last two to the "model parameters" of the model in this document. The model parameters are abstracted from the traces of events.

The post-mortem analysis of the events log starts by constructing the execution graph. Despite the crude information of the events log, the execution graph directly presents the 
causal or independence relationship between the events. The execution model is used during the subsequent stages of analysis to identify the software architecture and determine the model parameters. The execution graph also helps to formalize the process of performance model construction, irrespective of the processes used to create the execution graph. This fact suggests that an execution graph can also be used to translate other formal approaches of model building, such as PUMA (Performance from United Model Analysis) [62], to WebTime and vice versa.

The analysis of an execution graph to construct the performance model is performed in two different domains which are called the "events" and the "tasks" domains here. In the events domain, the tasks and the entries, as well as the event patterns representing interactions between the tasks, named here the "event interactions" or "EIs" of the execution graph, are identified. In the tasks domain, the EIs are categorized into classes of interactions, named the "tasks interaction" or the "TIs". In fact, in the events domain, the model architecture is constructed and, in the tasks domain, the model parameters are determined.

The remaining information needed to construct an LQN model are the CPU information such as multiplicity, scheduling discipline, speed and their mapping to tasks. Identification of these parameters is dependent upon the OS and the tools the OS provides to the instrumentation system. WebTime is designed to process these parameters and plug the results into appropriate places in the final LQN model. 
In summary, WebTime uses the processing step model of Figure 3-1 to construct an LQN model from an events log. These steps are listed in the order in which they are processed.

1. WebTime requires the availability of an executing application in order to construct the application's LQN model. Currently, the source code of the application should be available since instrumentation is performed manually.

2. An application should be instrumented, either manually or through an automated process, in order to generate event-traces. Instrumentation consists of inserting sensors and implementing a monitoring system in order to record event-traces.

3. An event-trace is a space delimited text which contains information about the task and the CPU in which the event was executed, as well as the logical clock time specification at which the events occurred, called the event's timestamp. Timestamps help to order the events.

4. In the first step of analysis, WebTime identifies the causal and independence relationship, between the event-traces of the events $\log$ of an invocation. At this step an event-trace is transformed into a different form which is called an "event of the execution graph" or simple an event. The result of this analysis is a set of connected events based on the order in which the events have occurred, which is called the execution graph. This process is explained in Chapter 4. 
5. From the execution graph the specifications of the tasks of a software application and their interactions will be identified, as explained in Chapters 5 and 6.

6. WebTime analyzes the specifications of the tasks and their interactions to construct the sub-models of invocations and eventually the final LQN model.

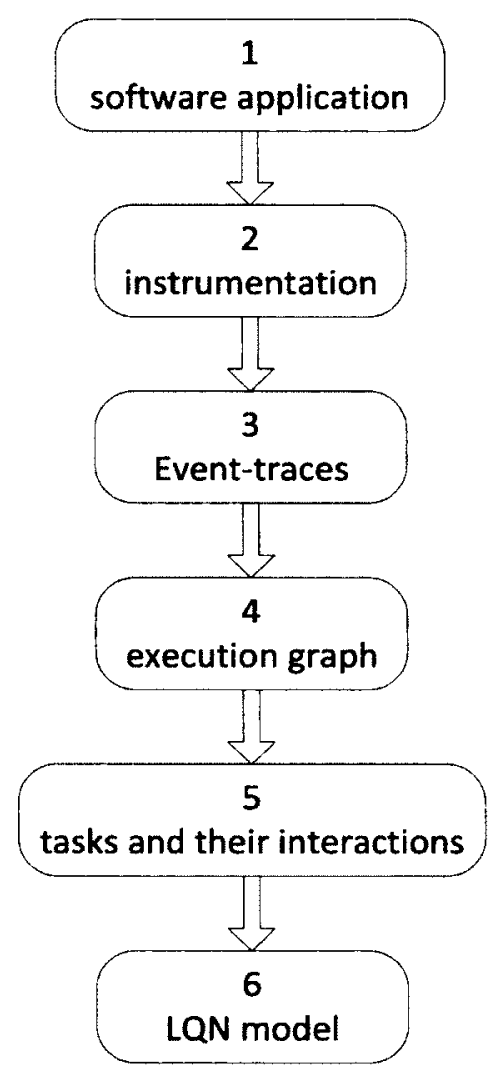

Figure 3-1 automated LQN model building process 


\subsection{Phases and activity graph}

There are two versions of an LQN performance model: "phases" and "activity graph". In the "phases" model, a task is characterized by one or two phases of operations by which all the task's behaviours are summarized. The "phases" model will be referred to as the "LQN performance model" or briefly "model" throughout this document. In the activity graph model all the details of the internal behaviours of a task are represented by activities and their interrelationships. The activity graph model will be referred to as the "activity graph" throughout this document. This research addresses the construction of both versions of the LQN model.

\subsection{Scope}

WebTime is concerned with incorporating the current state of a computing system into an LQN performance model for subsequent study to guide design modification and improvements, instead of providing suggestions as how to improve the design. WebTime can be used in a software performance engineering (SPE) fashion [3] in which a performance model is constructed and updated with the evolution of the software under design to avoid design pitfalls in all the stages of the development cycle.

WebTime is able to identify various interaction types in a client-server distributed system, thus is limited to identify interactions between two or more tasks which an LQN model can accept [56]. These interaction types include Synchronous, Asynchronous and Forwarding interactions, as well as loops of these types of interactions. WebTime can capture and characterize the concurrent operations in a task, which is the weakness of both Vector-Time [14] and Proper-Time [12] approaches. 
Chapters $4,5,6$, and 7 provide a detailed description of WebTime framework. Chapter 4 covers construction of the execution graph from events log. Chapter 5 explains the events domain analysis. The end result of this analysis is the creation of four vectors, as collections of objects, namely the CPU, entry, task and EI vectors. Chapter 6 is devoted to tasks domain analysis, which mainly extracts the model parameters of an execution graph. This chapter also explains the basis by which WebTime is considered a scalable framework. Chapter 7 uses the execution graph to automatically construct the activity graph of an invocation. 


\section{Chapter 4: Execution graph}

An execution graph is the next level of abstraction from that of an events log in which the relationship between the events of a distributed application are defined in a formal way. An execution graph is constructed from the events log of a computation to simplify and formalize the processing steps to construct the final LQN model from an execution graph. Figure 4-1 shows the processing steps to construct an execution graph.

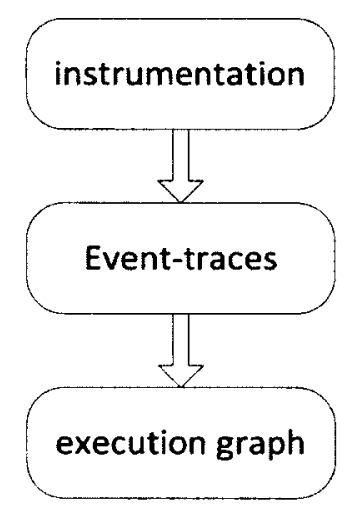

Figure 4-1 execution graph building process

\subsection{Characterization of an event in the execution graph}

An event in the execution graph is characterized based on its position in this graph. This event also carries some information about the system in which the event was 
executed which will be used in the other steps of the model construction process. An execution graph represents an ordered set of events of a computation. An execution graph can also be viewed as a number of threads in which events have linear relationships. These threads are not necessarily the real threads of a system but are determined based on the way in which an instrumentation mechanism is designed to relate these events. The threads of an execution graph are called the "eg_threads" to make this distinction. Each eg_thread has a parent eg_thread except the root eg_thread. An eg_thread represents a single sequential flow of control, as determined by the instrumentation system. Section 4.3 describes how these eg_threads are determined. Figure 4-2 shows the model of an event in an execution graph. An event in an execution graph may be preceded by one event and may also be succeeded by one or two other events. The first succeeding event, called the "internal successor", represents an event in the same eg_thread of this event. The second succeeding event, called the "external successor", represents an event in a different eg_thread. In fact the external successor is the first event of a new eg_thread which has just been spawned from the eg_thread of this event. The internal successors are shown at right side and external successors are shown at the top or bottom side of their originating events.

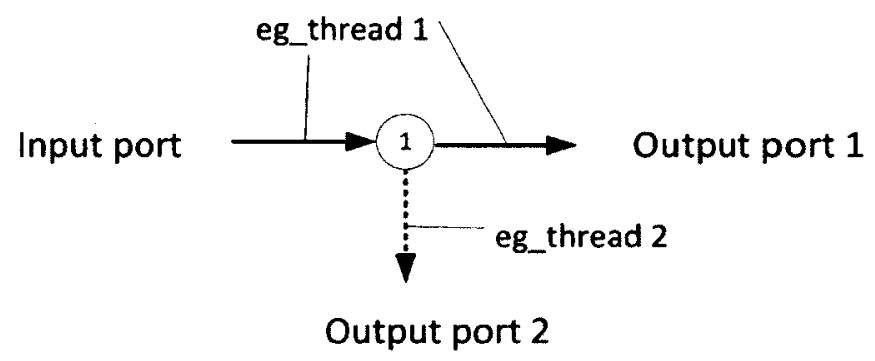

Figure 4-2 model of an event in the execution graph 
An event also carries a portion of an event-trace which contains the type of the event as well as the specification of the entities in which the events occurred including the name and the specification of the task, entry and CPU in which the event occurred. This portion of an event-trace, which is called the event's "profile" and is explained in the next section, is directly transferred from its event-trace into an execution graph event. Table 4-1 shows the attribute of an event.

Table 4-1 attributes of an event in the execution graph

\begin{tabular}{|c|l|}
\hline No & \multicolumn{1}{|c|}{ Attributes } \\
\hline 1 & The event's profile \\
\hline 2 & internal successor \\
\hline 3 & external successor \\
\hline
\end{tabular}

A simple example of the execution graph of a three-layer distributed application is shown in Figure 4-3. The events in a layer represent the events of a task. This execution graph is composed of four eg_threads which are shown using different line patterns. Eg_thread 1, which is the root, consists of events $1,2,3,4,5,6$ and 7 . Eg_thread 2 consists of events 8,9,12,13,14 and 17. Eg_thread 3 consists of Events 10 and 11 and eg_thread 4 consists of events 15 and 16. All the events have one input port and one output port except events 6,9 and 14 which have the second port for their external successor. These events are where eg_threads 2,3 and 4 spawn from eg_threads 1,2 and 2 respectively. As a result of receiving an external request, Task1 sends a request to Task 2 and blocks. Task 2 receives this request and makes a similar request to Task3. 
Task 3 then sends a reply to Task 2 by spawning the eg thread 2 and then performs its remaining operations, called a second operation phase. Task2 receives the reply and sends its own reply to Task 1 by spawning the eg_thread 3 and continues to a second phase in which it again interacts with Task3.

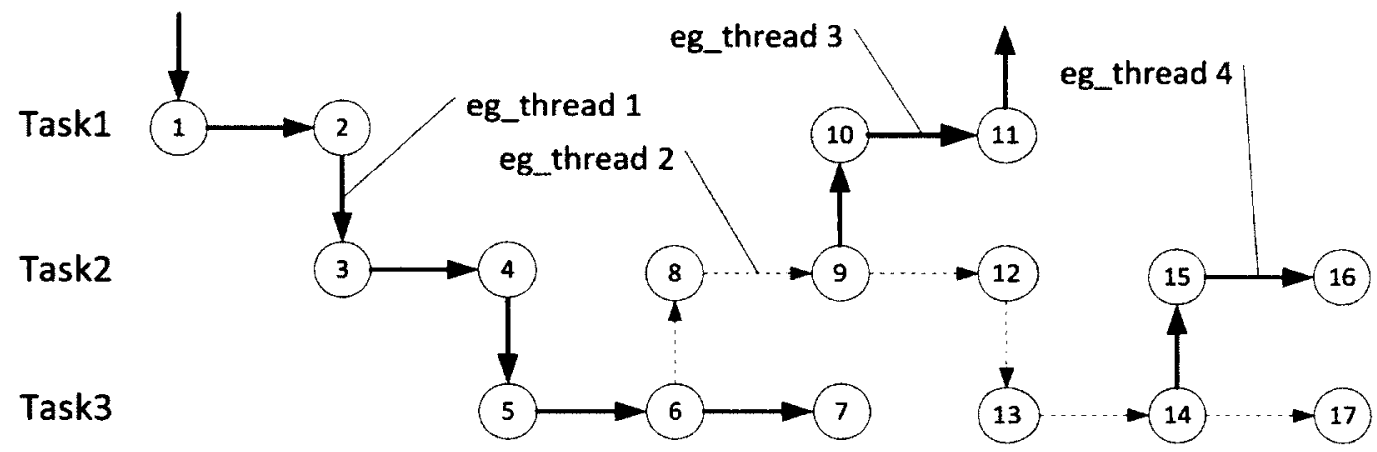

Figure 4-3 an execution graph - Task 2 and Task 3 perform second phase operations

The structure of the execution graph specifies the minimum information which should be incorporated into the content of an event-trace. The main elements of an execution graph are its events and eg threads. This explains that, in order to construct the execution graph, an event-trace must help to:

- Classify the events based on their eg_threads.

- Order the events which are classified in the same eg_thread.

- Relate the eg_threads of a computation to each other.

The structure of an event-trace entry is determined based on these requirements and is explained in the following section. 


\subsection{Event-trace structure}

The elements of an event-trace structure can be classified into two sub-structures i.e. the timestamp and the profile. The timestamp part of an event-trace is traced through the events of a distributed system and piggybacked on the messages passed between the nodes of that system. The timestamp is used to recover the causality of the events and helps to construct an execution graph. The profile sub-structure contains environmental information of an event including the task, its entries, the system in which the event was executed as well as the specifications of these entities. These parameters are locally obtained and don't require tracing through the events of a system. It is assumed that an event-trace contains all the required information to construct a performance model and also that event-traces are logged by a monitoring system which doesn't miss any events. One of the novelties of the event-trace structure is that the number of elements in the timestamp structure, which is sent between tasks, is very small.

\subsubsection{Timestamp}

The timestamp structure does not include a physical wall clock time and therefore does not require a clock synchronization mechanism which makes it suitable for a distributed application. In fact the timestamp structure is a new realization of a logical clock. It is assumed that the contents of a timestamp can be transferred by a message in a message passing system and the message communicating system is reliable but can carry messages in an arbitrary order. The small number of elements property of the timestamp structure reduces the communication overhead incurred associated with the timestamp load of the message. A timestamp consists of four fields: 
- Event index

- Invocation index

- Eg_thread index

- Parent eg_thread index

The first component of a timestamp is an event index. The index of an event will be incremented before it is saved in that event. The events of an eg_thread are indexed in the order in which they have been executed in that eg_thread. The event index of an event helps to find the internal successor of an event.

The invocation index classifies the events of a computation based on their invocations. An invocation is an independent unit of operation of a computing system which can separately be analyzed to assess the performance of that system. In general an assessment based on analysis of more than one invocation is more representative of the behaviour of an application. At an instance of time there may be more than one invocation running because some tasks may go to a second operation phase or the tasks may concurrently operate to respond to more demands. It is assumed that if a task maintains the state information about an invocation in a concurrent operation, it does not affect the state of other invocations.

The events of an invocation will also be classified based on the eg_thread in which they have occurred. The events in an eg thread have linear relationship i.e. they can be ordered based on their indices.

The parent eg_thread will be used to find the external successor of an event. 
In order to get an event properly indexed, a timestamp object is passed between the events of a computation. This object is modified by each event which receives that timestamp, logged and then passed to the following event. This is an effective means by which the causality of events can be maintained.

The timestamps are piggy backed on the messages passed between the tasks of a distributed application. This will affect the performance of the subject system by incurring communication overhead. The small size of a timestamp helps to reduce this overhead.

\subsubsection{Profile structure}

The profile section of the event-trace structure is used to identify the environment in which the events were executed such as the software resources and their attributes. The profile doesn't travel by the messages of a distributed application and so doesn't incur any communication delay. It is assumed the profile structure contains all the necessary information, in addition to the contents of a timestamp, to construct a performance model. These parameters include:

- the name of the task of an event

- the concurrency level of task

- the entry in which the event has occurred

- the type of the event

- the CPU in which the event occurred

- the multiplicity of the CPU 
- the net CPU-time consumed by the event

An instrumentation system is used to splice suitable code in a software system to allow for generation of the event-traces of that system when it runs.

\subsubsection{Event types}

Event types specify in which process an event has been executed and provide significant information to recover the architecture of a computing system. Event types are used to facilitate identification of various interaction types including Synchronous, Asynchronous, Forwarding and loops of Synchronous or Forwarding interactions. An interaction consists of two or more transitions between two or more tasks. The representation of the transition in an execution graph is called the "eg_transition". An eg_transition represents a directed arc between two events which belong to two different tasks in the execution graph. Various event types and their acronyms which are used in WebTime are shown in Table 4-2.

Table 4-2 event types and their brief description

\begin{tabular}{|l|c|l|}
\hline Event type & $\begin{array}{l}\text { Abbreviated } \\
\text { name }\end{array}$ & description \\
\hline Send & $S E$ & "send" event by a requesting task \\
\hline Receive & $R E$ & "receive" event by a server task \\
\hline Send acknowledge & $S A$ & Reply event in a server task \\
\hline Fork send & $F S$ & A non-blocking service request event \\
\hline Fork acknowledge & $F A$ & Non-blocking reply event in a server task \\
\hline end & $E N$ & Ending event of a eg__thread \\
\hline activity & $A C$ & ACPU-time consuming event \\
\hline external & $E X$ & Starting event of an operation \\
\hline
\end{tabular}


It is explained below that the way in which events are named allows:

1. Identification of a eg_transition from one task to another

2. Determination of the order in which the eg_transitions between two tasks have occurred in order to identify a complete interaction

In other words the intention is to use the types of events to identify an eg_transition and its direction. The direction of an eg_transition specifies if the eg_transition is from a client to a server task or vice versa. Only two oppositely directed eg_transitions may specify an interaction.

An eg_transition, from a client to a server, can be identified by an ordered set of event types. For example (SE, RE) specifies that an event of type SE in a client task has caused another event, in a sever task, which is of type RE. To make a distinction between two ordered set, at minimum, one component of the second one needs to be different. This distinction is made here by changing the type of the send event of a server task to SA, instead of SE, when it is replying to the request made to it. By this change the ordered set of event types representing the replying eg_transition will be specified by (SA, RE). A different example would be a situation in which a receiving task performs a second phase operation. In this case the task spawns a new eg_thread by using a fork event to reply to a request. This reply can be represented by either a FS or a FA type event. A FS type event is used to specify a forked request and a FA event is used to specify a forked reply. By this distinction it can be easily understood that a (FA, RE) can only be the second transition of an interaction and (FS, RE) the first. 
There are three other event types which associate with the internal operation in a task: "EX", "EN" and "AC". An "EX" event specifies the first event of an invocation which was issued by a reference task. An "EN" event indicates the last event of an eg thread. Finally an "AC" type denotes events which perform processing and consume CPU-time. In the diagrams in this document the acronyms of the event types, shown in Table 4-2, will be used.

\subsection{Instrumentation Mechanism}

The instrumentation system is responsible for annotating the events of an application to generate event-traces. The following assumptions are made when adding an instrumentation code:

1. An event in an application first records the timestamp it receives and then modifies the timestamp for its following events.

2. An event which doesn't have an external successor (event types of AC, SE, SA, RE or EX) only modifies its timestamp for its following event.

3. An event which does have an external successor (event types of FS or FA) both modifies its timestamp for its internal successor and also creates a new timestamp object for its external successor.

Only events which make asynchronous, or non-blocking, eg_transitions to other events have external successors. These events, which are either of FS or FA type, make their eg_transitions through a new eg_thread.

Modification of a timestamp consists of only incrementing the event index of the current timestamp. 
The creation of the new timestamp involves:

1. Cloning of the current timestamp to create a new timestamp.

2. Incrementing the event index of the new timestamp.

3. Setting the parent eg_thread index of the new timestamp to the eg thread index of the current eg_thread.

4. Generating a new eg_thread index for the new timestamp.

The only requirement for generation of the eg_thread index is that there shouldn't be any similar indices in an invocation. A high resolution random number generator can be used for this purpose. Figure 4-4 shows a simple implementation of the new timestamp creation process in Java.

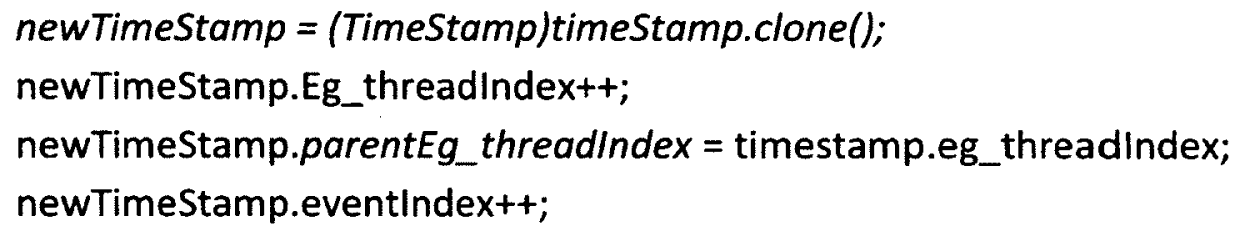

Figure 4-4 a java program to modify the timestamp of an even of type "FS" or "FA"

An example is used to illustrate the instrumentation mechanism for an asynchronous request. The left side of Figure 4-5 shows an asynchronous eg_transition from Event2, which is of type FS, to Event3. The instrumentation system creates a new timestamp from the original timestamp of Event 2 and also modifies the original timestamp of Event 2 and gets it passed to Event4. The new timestamp is then appended to the message which is 
sent to Event3. This can be compared with a blocking request (drawn in right side of Figure 4-5) in which the timestamp of Event 2 is only modified and sent to Event 3.
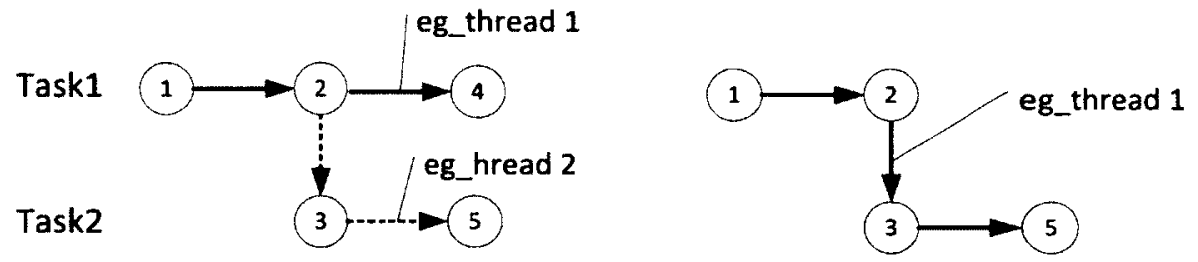

Figure 4-5 a non-blocking vs. a blocking service request

Another example is used to illustrate the instrumentation mechanism for an asynchronous response. The left side of Figure 4-6 shows an asynchronous eg_transition from Event2, which is of type FA, to Event3. Task2 performs a second phase operation after sending a response to Task1. The instrumentation system first modifies the timestamp of Event 2 and passes it to Event4. It then creates a new timestamp from the original timestamp of Event 2 and appends it to the message which is sent to Event 3 . This can be compared with a reply with no second phase (the right side of Figure 4-6) in which the timestamp of Event 2 is only modified and sent to Event 3 .
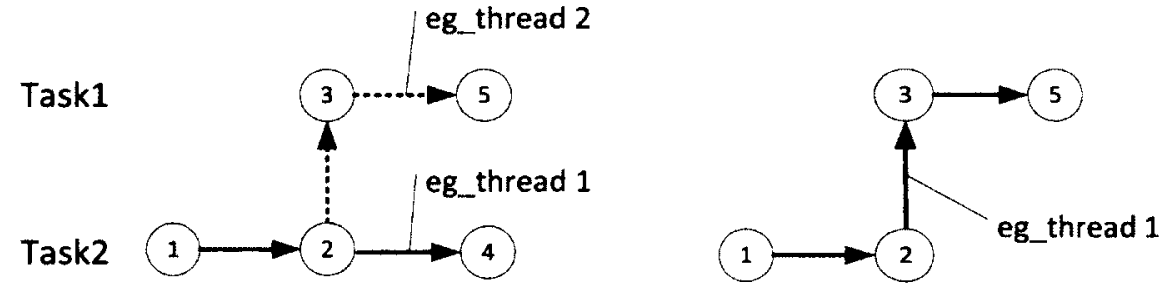

Figure 4-6 a reply with a second phase operation vs. a reply with no second phase 
It should be noted that the index of both the external successor and the internal successor of an event are identical and follow the index of that event, as shown in Figure 4-7. In fact these events can easily be distinguished by their eg_thread indices which are different.

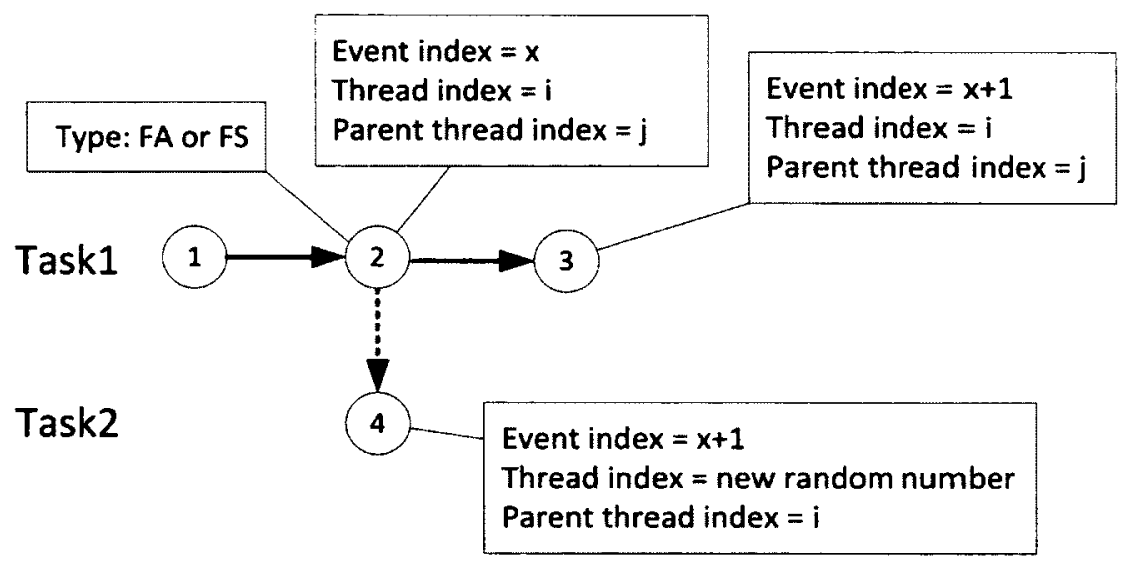

Figure 4-7 index of events following a fork event

Recording of the event-trace of an event consists of recording the parameters of the timestamp which is received by the events as well as the profile of that event. Figure 4-8 shows a program in Java which records the event-trace of an activity $(A C)$ event. The recording of other event types would be performed the same way except that the event type and the CPU-time consumption would be different. 


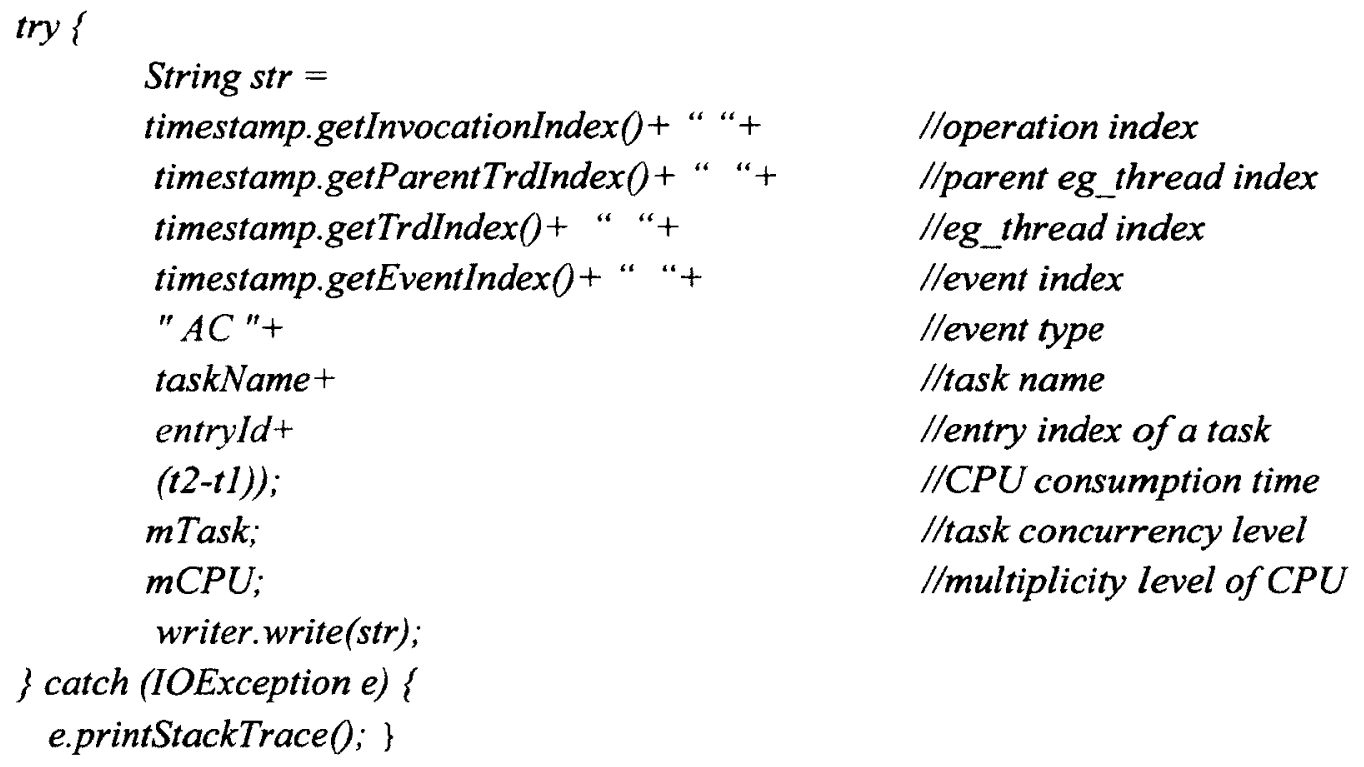

Figure 4-8 Java listing for an event recording

\subsection{Event-traces to execution graph transformation}

Transformation of event-traces into an execution graph involves finding the internal and the external successor of each and every event.

\subsubsection{Identification algorithm of the internal successor of an event}

In this algorithm, which is shown in Figure 4-9, the trace of an event, represented by Event $\mathrm{A}$, is compared against each of the other events in the same invocation. It is checked to determine if the two events meet the following conditions:

1. They both belong to the same invocation.

2. They both have the same eg_thread index.

3. The difference between their event indices is 1 . 
4. They have an acceptable type order, explained below.

IF event_A.invocation $==$ event_B.invocation
IF event_A.eg_threadg==event_B.eg_thread
IF event_B.index - event_A.index $==1$
IF (event_A and event_B have acceptable type order)
event_B is internal successor of event_A
IF END
$I F E N D$
$I F E N D \quad$ Figure 4-9 algorithm of determining if one event is internal successor of another

The acceptable type order between two internally ordered events is shown in Table 4-3.

Table 4-3 acceptable event type order between two internally ordered events

\begin{tabular}{|l|l|}
\hline event type & \multicolumn{1}{|c|}{ Possible succeeding event type } \\
\hline "SE" & " $R E$ " \\
\hline "SA" & " $R E "$ \\
\hline "RE" & " $A C$ " or " $S E$ " or " $S A$ " or " $F S$ " or " $F A$ " or " $E N "$ \\
\hline " $F S "$ & " $A C$ " or " $S E$ " or " $S A$ " or " $F S$ " or " $F A$ " or " $E N "$ \\
\hline " $E X "$ & " $A C$ " or " $S E$ " or " $F S$ " or " $E N "$ \\
\hline
\end{tabular}




\subsubsection{Identification algorithm of the external successor of an event}

The algorithm of identifying the external successor of an event is shown in Figure 4-10. In this algorithm the trace of an event will be compared with that of each of the other events in the same invocation. A match occurs when the two events meet the following conditions:

1. They both belong to the same invocation.

2. The eg_thread of the former event should be the parent eg_thread of the latter one.

3. The difference between their indices should be 1 .

4. They obey the type order, as will be explained shown in Table 4-4.

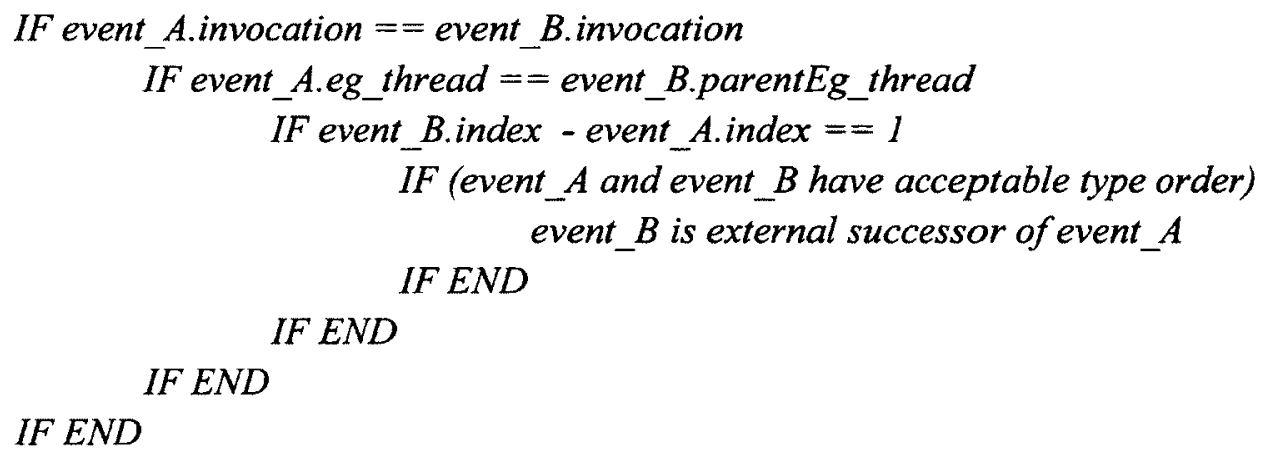

Figure 4-10 identification of external successor of an event 
Table 4-4 acceptable event types between two externally ordered events

\begin{tabular}{|c|c|}
\hline First event type & Succeeding event type \\
\hline "FS" & "RE" \\
\hline "FA" & "RE" \\
\hline
\end{tabular}

The computational complexity of this algorithm for constructing the execution graph of an invocation is $O\left(N^{2}\right)$. However since a computation usually consists of many invocations, the overall complexity of construction of execution graph of that computation is well below the overall quadratic complexity. The computational complexity of WebTime will be discussed in Chapter 6 . 


\section{Chapter 5: Event Interaction}

An interaction between two tasks can be either specified by the events involved in these tasks or, in a more abstract way, by the tasks themselves. The pattern of events which represents an interaction between two tasks is called here an "event interaction" or EI. When an interaction is specified by the involved tasks, it is called a "task interaction" or TI. The process flow to construct an LQN model from an execution graph is performed in two stages. In the first stage the EIs of the execution graph are identified. This stage is referred here as "events domain". More precisely, the identification of the tasks and their entries are also performed in the events domain but the characterization of the interactions between the tasks is not. In the second stage the identified EIs are abstracted to the TIs of that execution graph. This stage is referred to here as the "tasks domain". In the events domain the abstraction level of the identified EIs is raised by categorizing them into classes of interactions between tasks or TIs. Many of the fundamental attributes of a TI such as the phase in which the interactions have occurred, any nested interactions, and the CPU-time are determined by processing the identified EIs which are mapped to that TI. In fact, the events domain will be used as an engine to produce these specifications which are required to construct the LQN model of a computation. 
This chapter illustrates the processes used in the events domain to identify the EIs of an execution graph. The illustration of the processes used in the tasks domain is the subject of the following chapter. Five processing steps are used in the events domain to identify the various elements to construct the EIs of an execution graph which are shown in the diagram of Figure 5-1. In the next section, these elements and the way in which they are utilized to construct an EI will be briefly illustrated and, in the subsequent sections, these elements will be characterized in more details and the algorithm used for their identification will be explained.

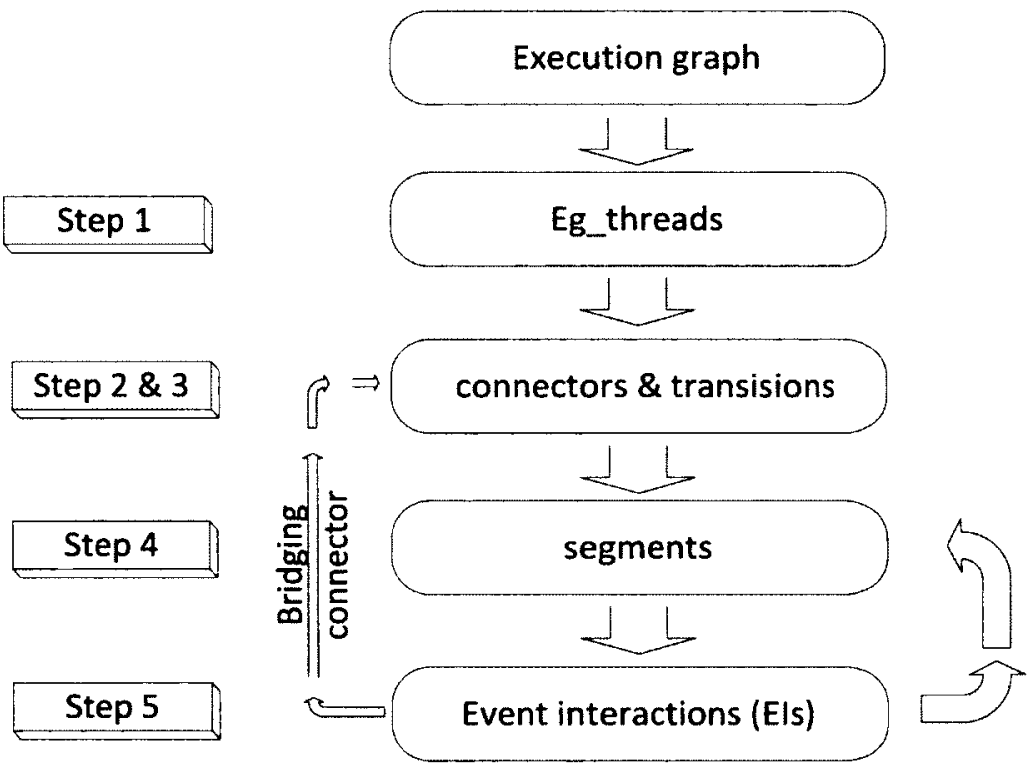

Figure 5-1 event interaction identification from execution graph diagram 
Throughout this chapter event types are identified by their acronym's which were defined in Subsection 4.2.3 and is repeated here for convenience of reading.

Table 5-1 event types and their brief description

\begin{tabular}{|l|c|l|}
\hline Event type & $\begin{array}{l}\text { Abbreviated } \\
\text { name }\end{array}$ & description \\
\hline Send & $S E$ & "send" event by a requesting task \\
\hline Receive & $R E$ & "receive" event by a server task \\
\hline Send acknowledge & $S A$ & Reply event in a server task \\
\hline Fork send & $F S$ & A non-blocking service request event \\
\hline Fork acknowledge & $F A$ & Non-blocking reply event in a server task \\
\hline end & $E N$ & Ending event of a eg_thread \\
\hline activity & $A C$ & ACPU-time consuming event \\
\hline external & $E X$ & Starting event of an operation \\
\hline
\end{tabular}

\subsection{Introduction}

In order to identify the EIs of an execution graph the abstraction level of the events of that execution graph will be raised in several steps until the abstraction level of the EIs is reached. The first step is to transform the events into two different patterns of events which are eg_transitions and the "eg_connectors" and are shown in Figure 5-2. An eg_connector represents a directed chain of links between the events of one single task. In fact an eg_connector may bridge the gap between two consecutive eg_transitions in an execution graph. Figure 5-3 shows, by breaking of an execution graph, the fact that an execution is actually constructed from eg_connectors and eg_transitions. An eg_transition and an eg_connector that share one event, as shown in Figure 5-4, will 
construct a segment. A segment is considered a special type of an eg transition to which an eg_connector is attached. This eg_connector is called the "tail" eg_connector of a segment.
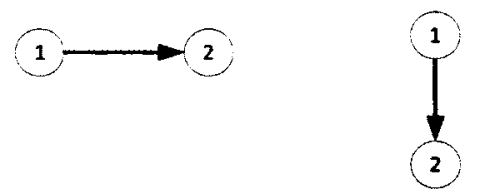

An eg_connector An eg_transition

Figure 5-2 an eg_connector and a eg_transition

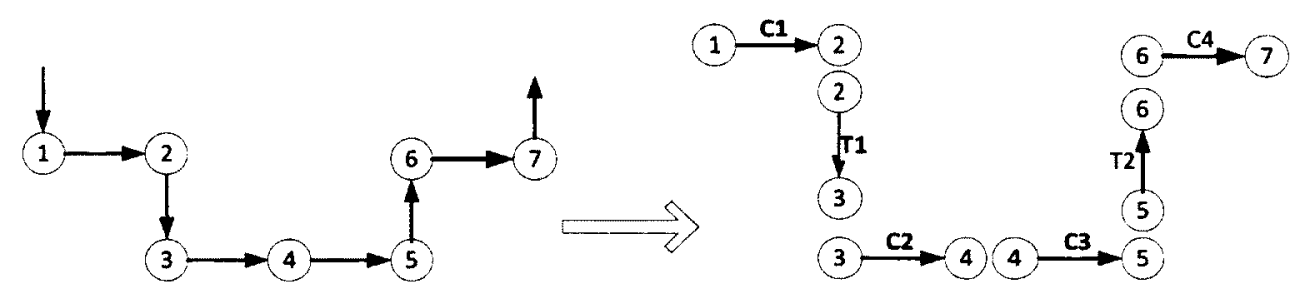

Figure 5-3 breaking of an execution graph into eg_connectors and eg_transitions

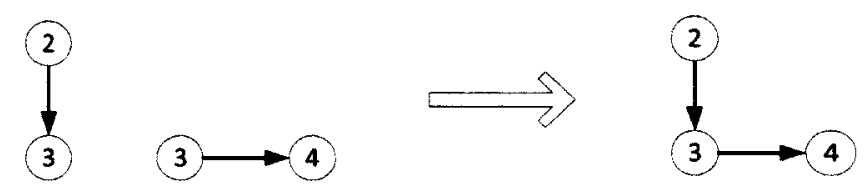

Figure 5-4 forming a segment from an eg_transition and an eg_connector

\subsubsection{Single EI}

A single EI represents an interaction between a client task and a server task in which the server doesn't make another (nested) interaction with a third task. A single EI is constructed using anti-parallel, matched and ordered set of two segments. These 
definitions are provided below. Figure 5-5 shows a single EI. The first event in a single EI is called the "head event" and the last event is called the "end event" of that EI.

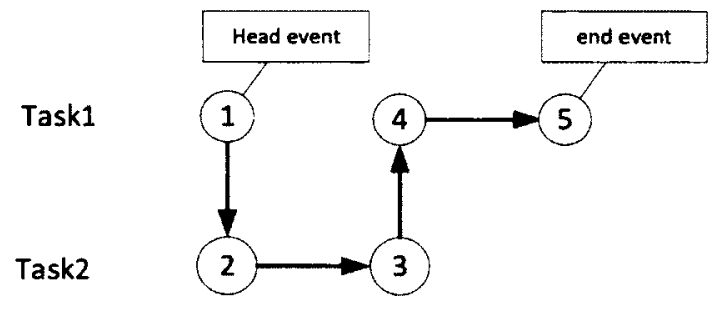

Figure 5-5 a single EI

Two segments are anti-parallel when the head event of one is executed in the same entry of that of the end event of the other and vice versa, as shown in Figure 5-6.

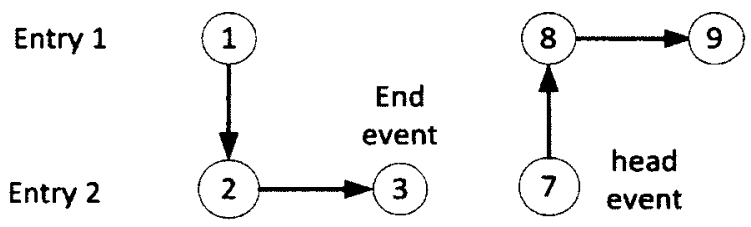

Figure 5-6 anti-parallel segments

Two segments are matched when the head event of one is shared with the end event of the other, as shown in Figure 5-7 in which Event $\mathrm{e}$ is shared between the two segments. 


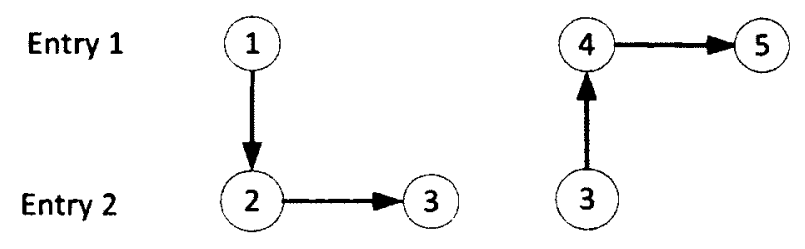

Figure 5-7 matched and anti-parallel segments

Two segments are ordered when the first segment is an initiating and the second one is an acknowledging type. In Figure 5-7, if Event1 is of type SE or FS and Event3 is of type SA or FA, the first segment would be of initiating and the second segment of acknowledging type and thus these two segments would be ordered.

\subsubsection{Clustered EI}

Often EIs will occur as a cluster of consecutive single EIs. A clustered EI is a set of consecutive single EIs in which the end event of each single EI is the head event of the following single EI, as shown in Figure 5-8. The number of single EIs in a clustered EI is called its "single-EI count".

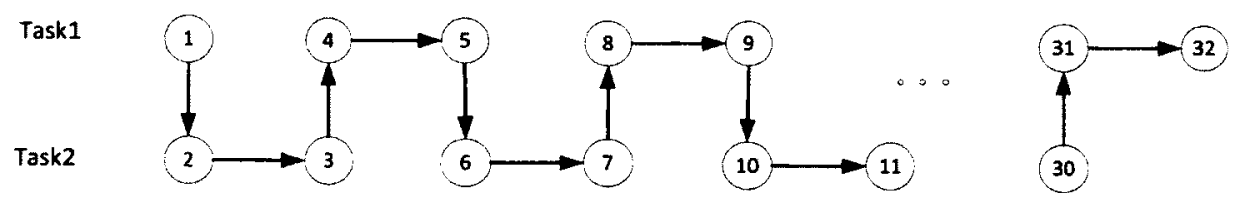

Figure 5-8 a clustered EI 
In this document the "EI" refers to the general concept of an event interaction which is a clustered EI. A single EI is a special form of an (clustered) EI in which the count of single EIs is one. The expression "single EI" will explicitly be used if a single EI will be referred to in this document. An EI is a representation of an interaction between tasks in the events domain which will be used to construct a TI. This EI will contribute to build up the necessary attributes of the EI including the CPU-times of the El's tasks and the El's interaction count. The next chapter covers this subject.

\subsubsection{Identification of Nested single EIs}

When an EI has a nested EI too, it will be first transformed into a single EI, as will be explained. After identification of the single and clustered EIs of a computation, which occurs at the two bottom layers of a hierarchical computation, each identified EI will be replaced with an eg_connector which bridges over the entire EI, as shown in Figure 5-9. This eg_connector will be merged into the eg_connector which precedes it and as a result will transform the nested EI to a single EI. More details about replacing of an EI with an eg_connector are provided in Sub-section 5.3.1 and about the merging of two eg_connectors in Sub-section 5.3.3. 


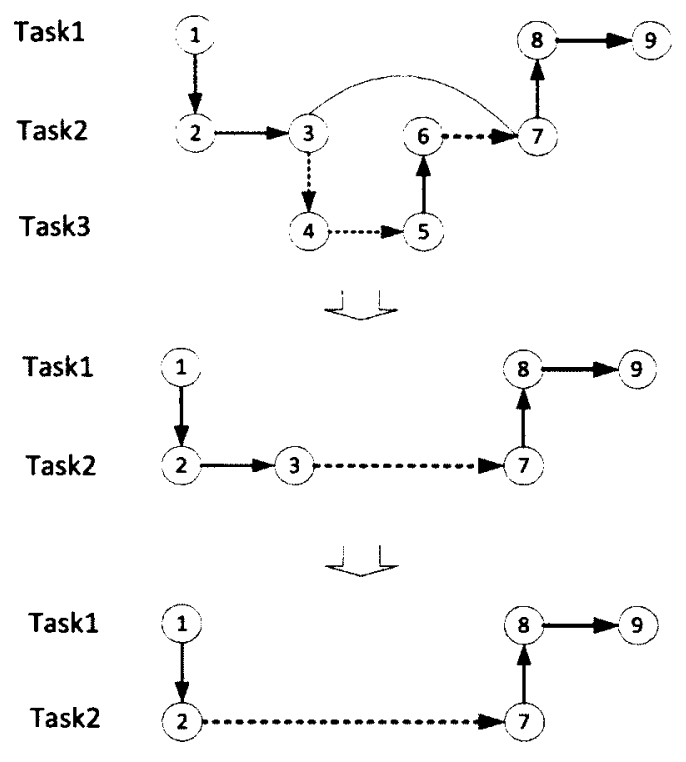

Figure 5-9 transforming a nested EI into a single EI

\subsection{Identification of eg_threads}

One way to construct the eg_connectors and eg_transitions of an execution graph is to walk through the sequence of events and to examine the type of each event and its internal and/or external successors. In order to make sure that all the events are visited, the events walking part of this operation is performed in two stages. In the first stage the eg threads are identified and their start-events are recorded in a vector, called "startevent". In the second stage the events of each eg_threads are examined by following through the internal successor of each event, from the start all the way to the last event. The start-event vector will be used by both an eg_connector and an eg_transition identification algorithm. The vector of start-events will contain an event of type EX and a number of events of type FS and/or FA. The algorithm of creating the vector of startevents is shown in Figure 5-11. This algorithm simply looks at the type of each event of 
an execution graph and puts the EX, FS and FA event types into the start-event vector. Figure 5-10 shows an example of an execution graph whose eg_threads of events are specified by alternate line patterns and its list of starts-events is shown in Table 5-2. It can be seen that there are four eg_threads in this execution graph. Eg_threadl starts with Event1 which is of "EX" type. The external successor of a fork event from which a new eg_thread is spawned specifies the starting event of that eg_thread. However, in these eg_threads, the fork events themselves are specified as the starting event because otherwise the eg_transitions from the fork events to their external successors would have been missed in the eg_transition identification algorithm. In this example, the starting events of Eg_thread 2, 3 and 4 are Events 1,6,9 and 14 respectively, as shown in Table $5-2$.

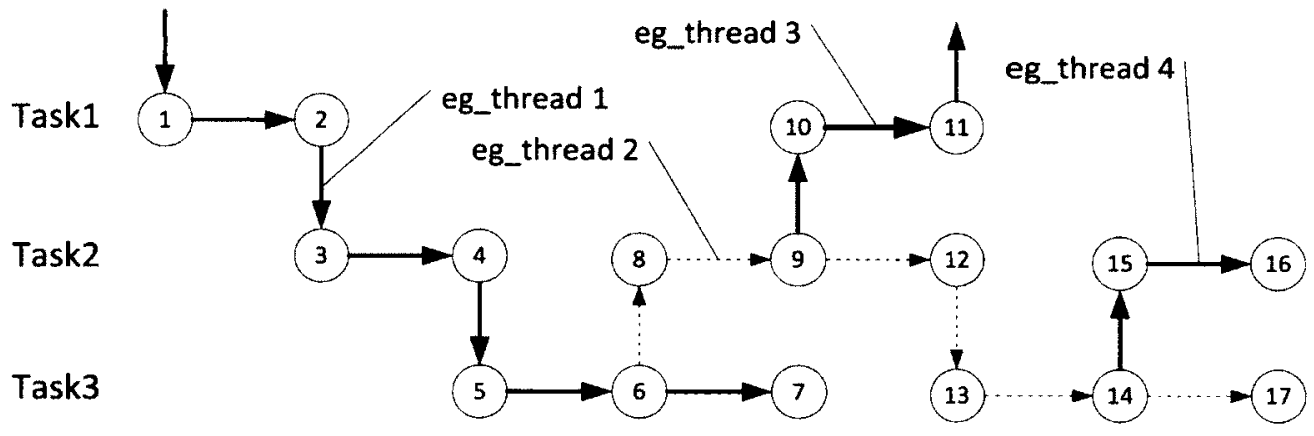

Figure 5-10 specifying the threads of an execution graph 
Table 5-2 start-events of the threads in Figure 5-10

\begin{tabular}{|c|c|c|}
\hline Thread & Start-event & type \\
\hline Thread1 & 1 & $E X$ \\
\hline Thread2 & 6 & $R E$ \\
\hline Thread3 & 9 & $R E$ \\
\hline Thread4 & 14 & $R E$ \\
\hline
\end{tabular}

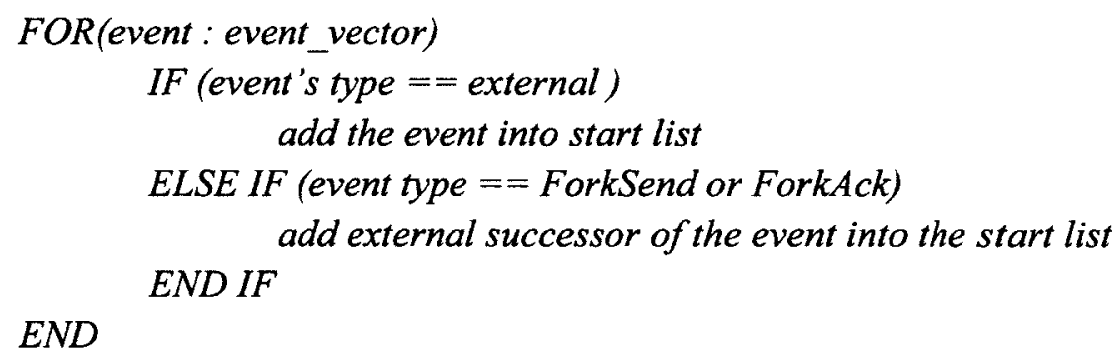

Figure 5-11 algorithm of finding the start-events of an execution graph

\subsection{Eg_connectors}

An eg_connector has an important role in identification of the phase in which an EI has occurred. For this purpose eg_connectors need to be characterized in much more details. The definition of the eg_connector in Section 5.1 helped to understand the definitions of Els but for the rest of this chapter more details are required. An eg_connector is formed by a single sequential flow of control in a task which is not branched. In other words an eg_connector is a straight (no branching in between) mediator between: 
- The end of an eg_transition to a task (RE) and the start of another eg_transition (FS, FA, SE, SA)

- The end of an eg_transition (RE) to the end event of a eg_thread in the same task (EN)

- A fork event (FS, FA) to the start of another eg_transition in the same task ( FS, FA, SE, SA)

- A fork event (FS, FA) to the end event of a eg_thread in the same task (EN)

The types of events in an execution graph can be used to identify the head and end events of an eg_connector. Table 5-3 shows the potential event types which could represent the start and the end event of an eg_connector. Figure 5-12 shows some possible patterns of events in which an eg_connector can be identified to explain the type of the start and the end events of an eg_connector. In this figure the events are represented by their types.

Table 5-3 potential event types for head and end events of a connector

\begin{tabular}{|c|c|}
\hline Identifier of: & Event type \\
\hline Connector head & "RE", "FS", "FA" or " $E X "$ \\
\hline Connector end & "FS", "FA", "SE", "EN" or " $S A$ " \\
\hline
\end{tabular}



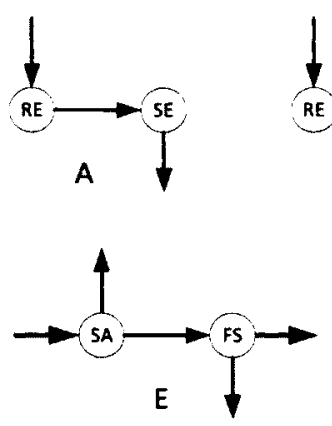

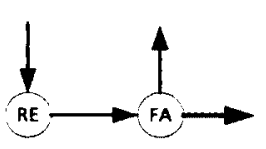

B

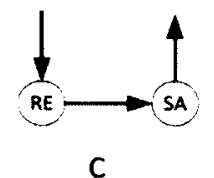

C

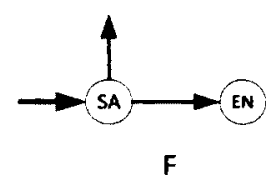

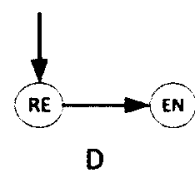

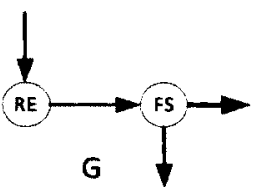

Figure 5-12 possible positions of an eg_connector in the execution graph

\subsubsection{Characterization of an eg_connector}

The main attributes of an eg_connector are shown in Table 5-4.

Table 5-4 attributes of a connector

\begin{tabular}{|l|l|}
\hline No & Attribute \\
\hline 1 & Head event \\
\hline 2 & End event \\
\hline 3 & Id of the bridged EI, EI-id \\
\hline 4 & CPU-time vector \\
\hline 5 & Type \\
\hline
\end{tabular}

- The "head" and the "end" events are the starting and ending events of an eg_connector.

- An eg_connector may carry the ID of an EI which, in the process of EI identification, was attached to it.

If an eg_connector carries an EI-id, it is either a replacement of an EI or has been merged by an EI replacement eg_connector, as was shown in Figure 5-9. Figure 5-13 
shows the mechanism by which the ID of an EI is transferred to its replacement eg_connector which bridges over the EI to simplify the EI identification process. The EIid attribute will later be utilized to identify the operation phase of that EI.

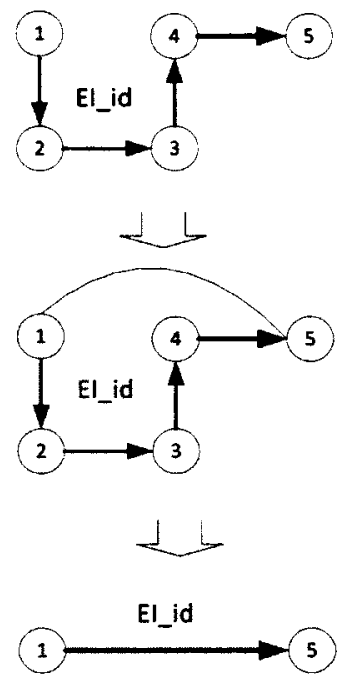

Figure 5-13 transfer of an EI-id to theeg_connectorwhich collapses it

- The "CPU-time vector" contains the CPU-times of the all the events of an eg_connector.

- The "type" specifies the position of the eg_connector in an EI structure.

The eg_connectors in different sections of an EI have been given different names since they will be used to identify various attributes of that EI. As it is shown in Figure 5-14, the eg_connector between an EI and its second phase nested EI is named as "second 
phase" or "SP". The SP eg_connector of an EI helps to identify a nested EI which has been performed in the second phase of the main EI.

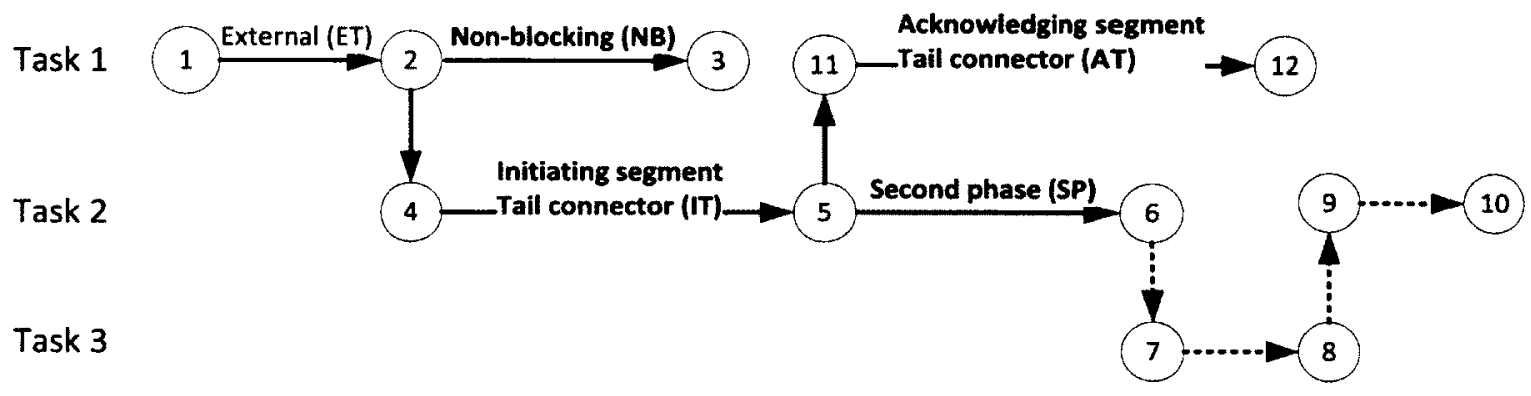

Figure 5-14 naming of connectors in different sections of an EI

In a non-blocking request for service, a client task continues its internal operation before blocking for a response from the server task. The eg_connector which represents this continued operation of the client task is named here as a "non-blocking" eg_connector or "NB".

The first eg_connector of Figure 5-14 whose head event is of type "EX" is named "external" eg_connector or "ET". There is only one "ET" eg_connector in an invocation.

A synchronous interaction consists of two matching segments which one of them initiates the interaction and the other one responds or acknowledges to the client. The eg_connector of each of these segments is named a "tailing" eg_connector. The tailing eg_connector of the initiating segment is named here "initiating tail" eg_connector or "IT" and the tailing eg_connector of the acknowledging segment is named “acknowledging tail" eg_connector or "AT". 
A "bridging", or "BR", eg_connector is made up during the EI identification process to facilitate analysis of nested EIs as was shown in Figure 5-13. A“BR" eg_connector is a two-event eg_connector consisting of only the head and the end events of the EI with which it replaced.

Table 5-5 summarizes the list of all eg_connector types in an execution graph with a short description of their application in constructing the LQN model of an operation.

Table 5-5 various eg_connector types in an execution graph

\begin{tabular}{|c|c|l|}
\hline Connector type & Abbreviation & \multicolumn{1}{|c|}{ Short description } \\
\hline Second phase & $S P$ & Used to identify second phase EIs \\
\hline Non-blocking & $N B$ & Used to count for CPU consumption time \\
\hline Initiating tail & $I T$ & Used to identify first phase EIs \\
\hline Acknowledging & $A T$ & \\
\hline External & $E X$ & Used to identify the first eg_connector of $a$ \\
\hline Bridging & $B R$ & Used to identify nested EIs \\
\hline
\end{tabular}

\subsubsection{Eg_connector identification algorithm}

The algorithm of Figure 5-15 is used to identify the existing eg_connectors in one eg thread of an execution graph. The algorithm starts by taking the first event of the eg_thread. It records the event as the head event of the first eg_connector to be identified and then walks through the events by following each event's internal successor. It stops where there is an eg_transition (a "FS", "FA", "SE" or "SA" type event) or an "EN" type event. At this point the "end" event of the first eg_connector is identified. If the event at 
this point is of type "FS", or "FA", the algorithm records that event as the head event of the following eg_connector which would be of type NB or SP, respectively. Instead if this event is of type "SE" or "SA", then that event would be the start of an eg_transition, therefore the algorithm moves ahead in the eg_thread until finds the start of a new eg_connector which would be an event of type "RE". It takes that point as the head of the following eg_connector and continues the same way to identify the corresponding end event. This continues until all the eg_connectors of that eg_thread are identified, which is when the last event of that eg_thread has been reached. This algorithm will be invoked for every eg_thread of an execution graph whose first event was already placed in the start-event vector by the algorithm of Figure 5-11. The end result of the application of this algorithm is the identification of all the eg_connectors in an execution graph and the recognition of types of SP and NB eg_connectors. The identified eg_connectors will be put in a vector called "eg_connector vector". 


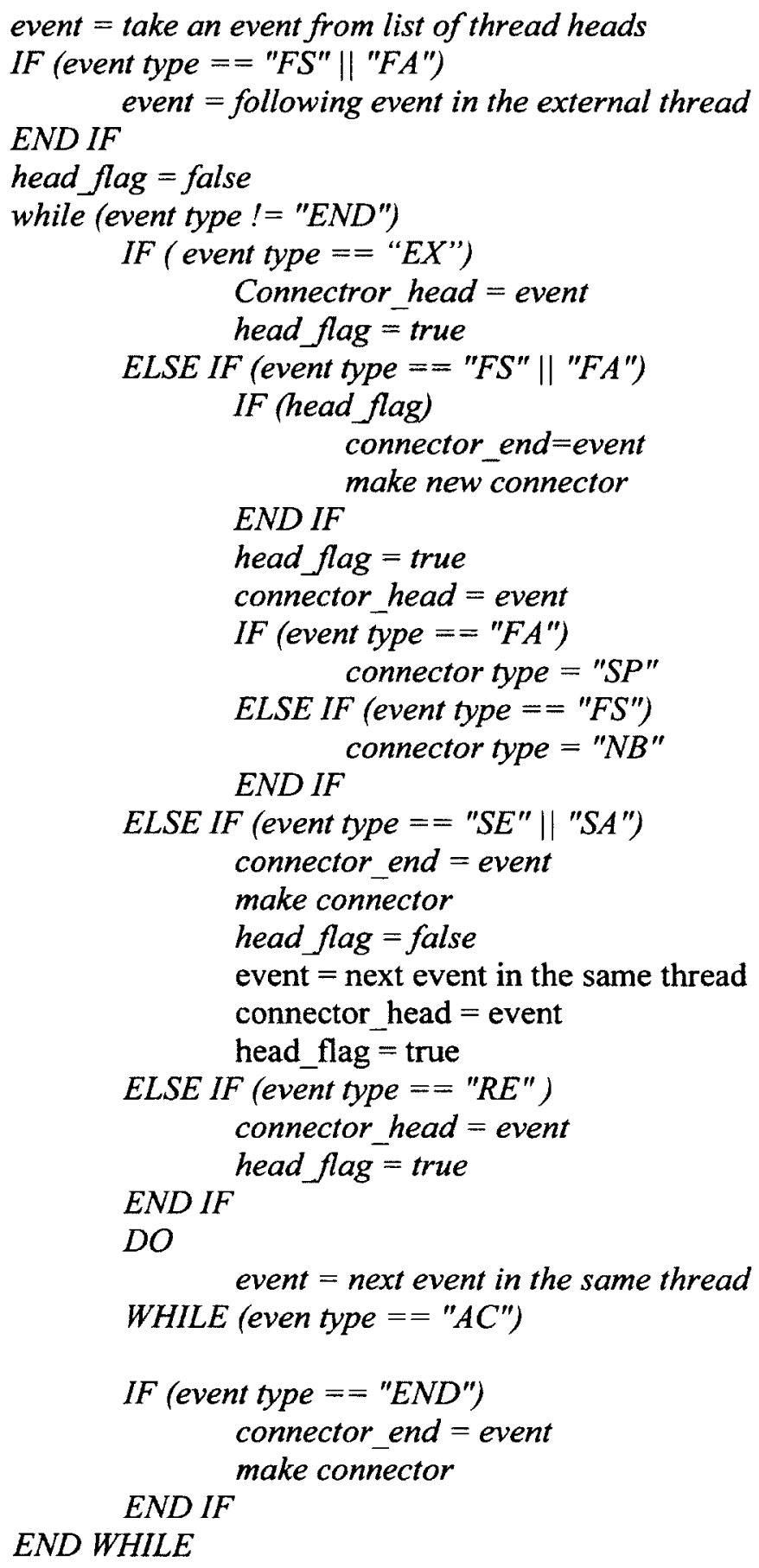

Figure 5-15 eg_connector identification algorithm 


\subsubsection{Merging of two eg_connectors}

There are situations where it is needed to merge two consecutive, and in the "same direction", eg_connectors to one, as shown in Figure 5-16. The first eg_connector is called the "merging" eg_connector and the second one the "merged" eg_connector.

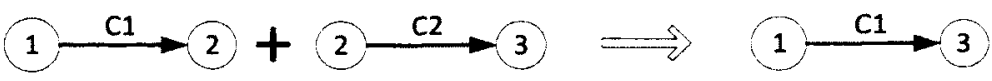

Figure 5-16 merging of two connectors

Two eg_connectors are consecutive if the tail event of the first eg_connector is the head event of the second one. When the two eg_connectors are merged, the second eg_connector will be collapsed and its attributes will be used to update those of the first one, such as:

- The merged eg_connector keeps its type, ID and head event

- The EI-id of the merging eg_connector is transferred to the merged eg connector

- The end event of the merged eg_connector will be changed to that of the merging eg_connector

The merging of two eg_connectors happens only when the second eg_connector is of type BR. This operation is part of the nested EI identification process that was explained in Figure 5-9. Figure 5-17 shows the algorithm for merging two eg_connectors. 
FOR (connectorl : connector_vector)

FOR (connector2 : connector_vector)

IF ( connector 2 is of type BR

$A N D$

end event of eg_connector $I==$ start event of connector2)

-change end event of Connector 1 to that connector 2

-change EI-id of connectorl to that of connector 2

IF END

FOR END

FOR END

Figure 5-17 algorithm of merging two connectors

\subsection{Eg_transitions and segments}

When flow of control is transferred from one task to another, an eg_transition has taken place. In fact, an eg_transition represents a one-way interaction between the events of two different tasks. An eg_transition is characterized by the attributes shown in Table $5-6$.

Table 5-6 attributes of a transition

\begin{tabular}{|c|l|}
\hline No & Attributes \\
\hline 1 & Head event \\
\hline 2 & End event \\
\hline 3 & Type (initiating or acknowledging) \\
\hline 4 & Tail eg_connector id \\
\hline 5 & NB eg_connector id \\
\hline 6 & SP eg_connector id \\
\hline 7 & Upper CPU-time vector \\
\hline 8 & Lower CPU-time vector \\
\hline
\end{tabular}


1. The head event specifies the sending event of an eg_transition.

2. The end event specifies the receiving event of an eg_transition

3. The type of an eg_transition specifies whether it is initiating a request to a task or it is responding to a request. In the former case the eg_connector is of "initiating" type and in the latter case it is an "acknowledging" type.

The relationship between the types of the events of an eg_transition and the type of the eg_transition itself is specified in Table 5-7. The head event of an initiating eg_transition is of type "SE" or "FS" and that of an acknowledging eg_transition is "SA" or "FA".

Table 5-7 relation between the type of a transition and its head and end events

\begin{tabular}{|c|c|}
\hline head-end event & Transition type \\
\hline$(S E, R E)$ & initiating \\
\hline$(F S, R E)$ & initiating \\
\hline$(S A, R E)$ & acknowledging \\
\hline$(F A, R E)$ & acknowledging \\
\hline
\end{tabular}

The types of the eg_connectors which may be connected to the head and end side of an eg_transition, called the attached eg_connectors of an eg_transition, are considered as the attributes of that eg_transition. The combination of an eg_transition and its attached eg_connectors, as will be seen in the following sections, help to identify and characterize the EIs of an execution graph. Figure 5-18 shows the attached eg_connectors of an 
initiating and an acknowledging eg_connector. By considering that an eg_transition is part of an interaction between a client and a server tasks, the NB and AT attached eg_connectors contain the activities of the client task and the IT and SP eg_connectors contain those of the server task. For this reason the AT and NB eg_connectors are called the client eg_connectors, and the SP and IT eg_connectors are the server eg_connectors. The various types of the attached eg_connectors of an eg_transition are specified below, in the same order as they are defined in Table 5-6.

4. The tail eg_connector specifies the eg_connector which is connected to an eg_transition at its end event side. In an initiating eg_transition, this eg_connector is of IT type and in an acknowledging eg_transition it is of AT type.

5. The NB eg_connector specifies the eg_connector which is attached to an initiating eg_transition at its head event side.

6. The SP eg_connector specifies the eg_connector which is attached to an acknowledging eg_transition at its head event side.

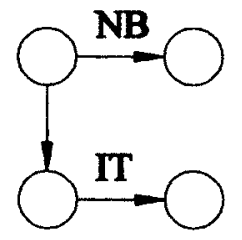

Initiating segment

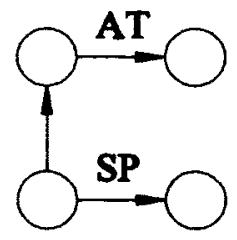

Acknowledging segment

Figure 5-18 complete form of various segments 
An eg_transition object is also equipped with two CPU-time vectors, named here as "upper" and "lower" vectors. These vectors are described below.

7. The upper CPU-time vector of an eg_transition contains the CPU-times of the events of either the NB or the AT eg_connector of an eg_transition.

8. The lower CPU-time vector of an eg_transition contains the CPU-times of the events of either the IT or the SP eg_connector of an eg_transition.

When an eg_transition is formed, the CPU-time vector of its client eg_connector will be incorporated into the upper CPU-time vector of that eg_transition and that of its server task will be incorporated into the lower vector of that eg_transition.

\subsubsection{Eg_transitions identification algorithm}

The eg_transition identification algorithm walks through every event of an execution graph to find the two events which have the required relationship to form an eg transition. The algorithm puts all the identified eg_transitions in a vector which is called "eg_transition vector". The walking paths of the algorithm are though the eg_threads of an execution graph. It takes one event from the vector of start-events at a time and walks through all the events of the corresponding eg_thread of that event. The algorithm follows through the internal successor of each event, except for the first event, until reaches to the "EN" event of that eg_thread. If the first event of the eg_thread is of type "FS" or "FA", the algorithm takes the external successor of that event and if it is of "EX" type the algorithm takes the internal successor of that event. Figure 5-19 shows the algorithm for identification of the eg_transitions of an execution graph. 
The method for isolation of each two events of an eg transition is explained here. The algorithm first finds a sending event and saves it as a potential head event of an eg_transition. This event would be of type "SE" or "SA". The algorithm bypasses all the events of types: "FS", "FA" and "AC" as it walks through the events and when reaches a receiving event, type "RE", then the two events of an eg_transition are identified and the eg_transition will be recorded. The type of an eg_transition will also be determined depending upon the types of the sending events, as was explained by Table 5-7 . 


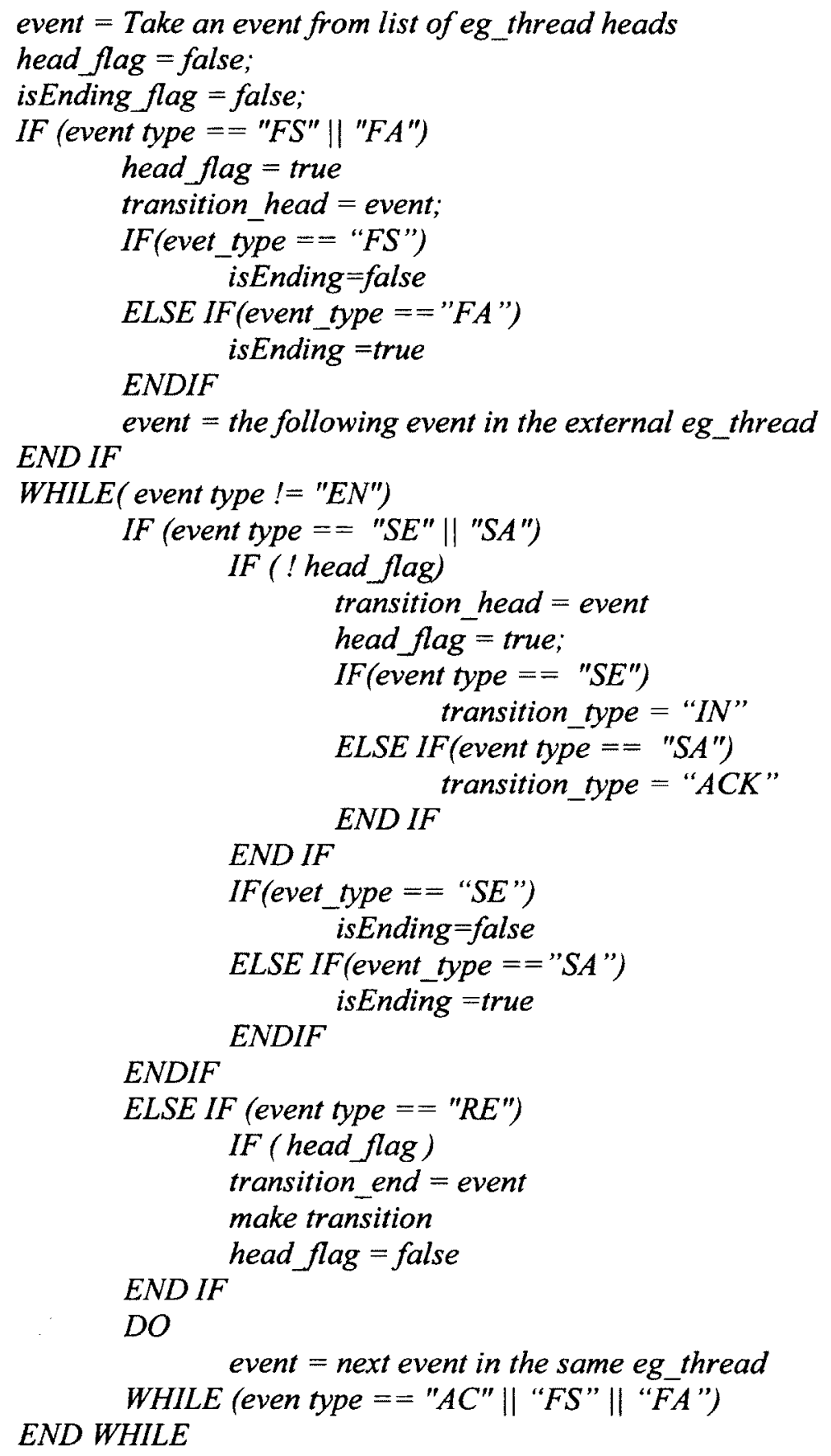

Figure 5-19 transition identification algorith m 


\subsection{Tasks and entries}

Tasks and entries are identified from the eg_connectors. Either the head or the end event object of an eg_connector will be used to extract the name of the task and the entry to which the eg_connector belongs. The task and the entry objects, when created, will be added to two different vectors named "task vector" and "entry vector" respectively. Since there would be more than one eg_connector associated with one particular task, the vectors are searched for pre-existing objects before creating a new one. A task and an entry are characterized by the attributes shown in Table 5-8 and Table 5-9 respectively.

Table 5-8 attributes of a task

\begin{tabular}{|c|l|}
\hline No & \multicolumn{1}{|c|}{ attribute } \\
\hline 1 & Name \\
\hline 2 & Vector of entries \\
\hline 3 & CPU \\
\hline 4 & Multiplicity \\
\hline 5 & Reference flag \\
\hline 6 & Vector of connectors \\
\hline
\end{tabular}

Table 5-9 attributes of an entry

\begin{tabular}{|l|l|}
\hline No & \multicolumn{1}{c|}{ attribute } \\
\hline 1 & Name \\
\hline 2 & number of incoming calls, at phase1 \\
\hline 3 & number of incoming calls, at phase2 \\
\hline 4 & Vector of CPU-times, at phasel \\
\hline 5 & Vector of CPU-times, at phase2 \\
\hline 6 & Optional : name of the task this is an entry of \\
\hline
\end{tabular}


It should be noted that tasks and their entries might operate over two different phases of operation. These phases are implicitly specified in the attributes of an entry. For example an entry's CPU-time vector and the number of incoming calls are specified separately for both phases. The attributes of Table 5-8 and Table 5-9 are as follows:

- Name is a string identifier for a task or entry.

- The vector of entries of a task contains the names of all the entries of that task.

- A task should always be mapped to a CPU as its processing unit. It is possible to map several tasks to a single CPU.

- The multiplicity attribute specifies the number of concurrent operations allowed in a task.

- An LQN model requires setting one task as the load generator or "reference" task of the model. A reference task is identified from the task in which an event of type "EX" is executed. When an eg_connector contains an event of type "EX", the reference flag of the task of that eg_connector is set or changed to "reference".

- The eg_connector vector of a task contains all the eg_connectors executed in the task. This vector will be used to construct the activity graph of a task. Activity graphs will be explained in Chapter 7.

- The number of incoming calls is a quantifiable number which can be determined from the number of calls made to an entry. This number will be used to determine the request rate of that entry.

- Two vectors of CPU-times, associated with the two phases of operation, are also designated to each entry to carry the CPU-time of each event executed in it. 


\subsection{CPUs}

The CPUs of a system are characterized by their name, their multiplicity and their scheduling discipline such as FIFO, random or pre-emptive.

Table 5-10 attributes of a CPU

\begin{tabular}{|l|l|}
\hline No & Attribute \\
\hline 1 & Name \\
\hline 2 & Multiplicity \\
\hline 3 & Scheduling discipline \\
\hline
\end{tabular}

A CPU object will be created from the attributes of an event and are placed in a vector called the "CPU-vector".

\subsection{Event Interactions (EIs)}

Els were discussed in Sub-section 5.1.1. In this section the EI characterization and identification algorithm are discussed.

\subsubsection{EI characterization}

An EI in its general form is characterized by the attributes shown in Table 5-11. 
Table 5-11 attributes of a clustered EI

\begin{tabular}{|c|c|}
\hline No & Attribute \\
\hline 1 & segment-set vector \\
\hline 2 & Upper CPU-times vector \\
\hline 3 & Lower CPU-time vector \\
\hline 4 & Type \\
\hline 5 & Phase \\
\hline
\end{tabular}

- The segment-set vector contains all the pairs of matched, ordered and anti-parallel segments of an execution graph.

- Each of the CPU-time vectors (upper and lower) contains the sum of all the elements of the corresponding (upper and lower, respectively) CPU-time vectors of the segments of the segment-set vector. The number of the single EIs in a clustered EI is simply number of the elements of the segment-set vector.

- An interaction type could be "Synchronous", "Forwarding" or "Asynchronous". In case of an asynchronous EI, there is no second element for each set of segments.

- The phase indicates in which phase the clustered EI has been executed.

\subsubsection{EI-identification algorithm}

The EI identification process is performed in a number of cycles in which three steps are involved. The number of cycles are $n-1$ times where $n$ is the number of hierarchical levels of interaction in a layered structure. In the first step of each cycle the identification 
of all the matched, ordered and anti-parallel pairs of segments will be performed to construct the single EIs of an execution graph. The resulting EIs will be put in a vector called here the "EI-vector". In the second step, the single EIs are merged into other EIs and removed from the EI-vector. Finally, in the third step, a bridging eg_connector for each clustered EI will be created. These eg_connectors are merged into other eg_connectors of the eg_connector-vector.

The algorithm of Figure 5-20 shows the first step of EI identification algorithm. Here, each two different segments of the eg_transition-vector are compared to determine if they are matched, ordered and anti-parallel segments. Every segment from the eg_transitionvector will be compared against all the elements of that vector by this algorithm to find its pair to form a single EI.

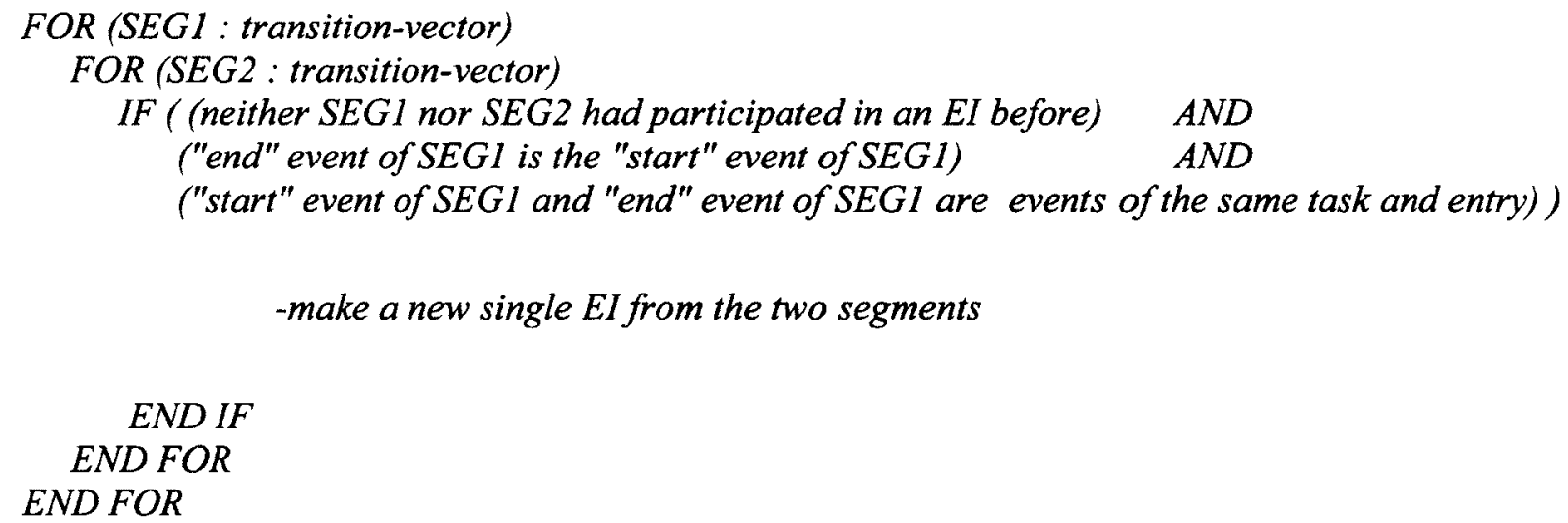


The second step of the algorithm is shown in Figure 5-21. This algorithm consists of two nested FOR loops which each independently take an EI from the EI-vector. The algorithm merges the EI of the inner loop to that of from the outer, if they satisfy the merging conditions.

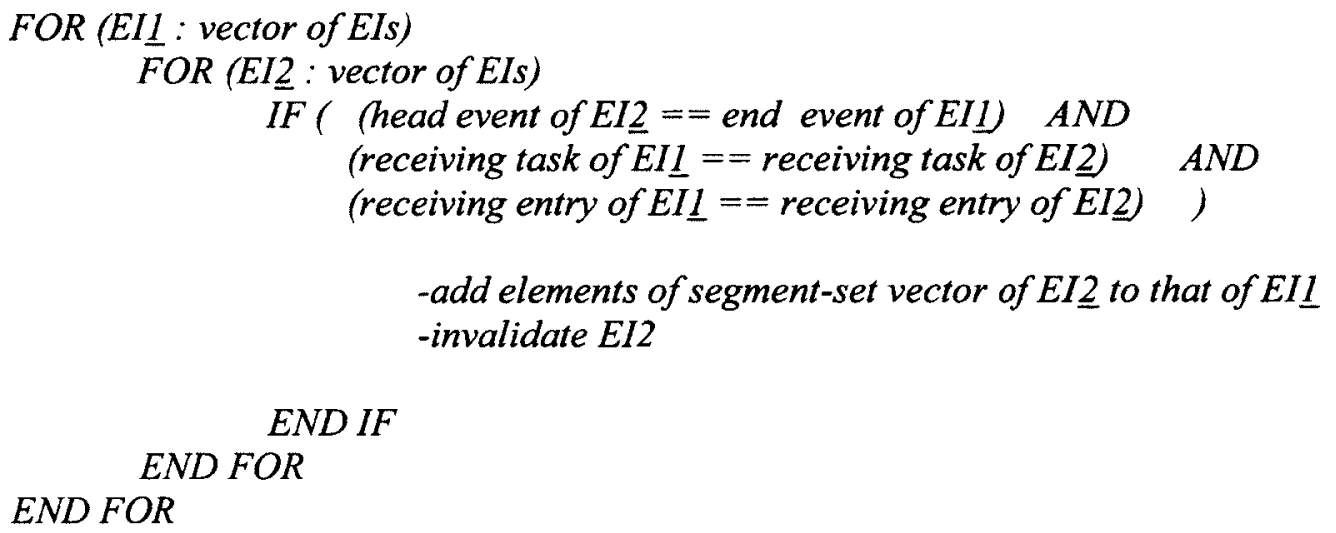

Figure 5-21 algorithm of merging consecutive single EIs to form a clustered EI

The second part of the algorithm creates a bridging eg_connector for each identified EI and attaches the ID of that EI to this eg_connector.

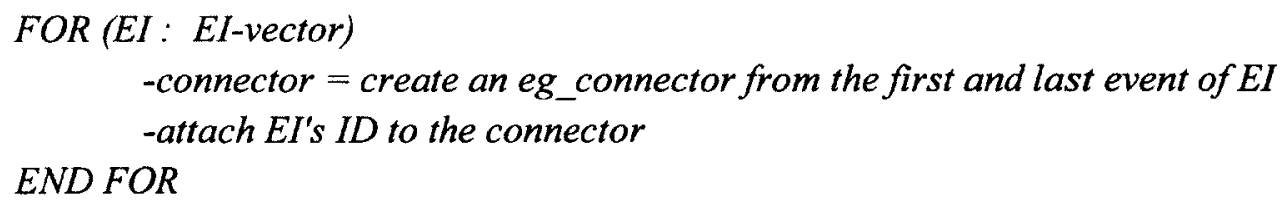

Figure 5-22 algorithm of creating the bridging eg_connector of an EI 
After merging the identified EIs together and the creation of bridging eg_connectors of the formed clustered EI, the algorithm of Figure 5-17 is used to merge the bridging eg_connectors to the real eg_connectors, which transforms the unidentified nested EIs to single EIs. The next cycle of running the algorithms of Figure 5-20, Figure 5-21, Figure 5-22 and Figure 5-17 will identify new EIs and transform them for the next cycle.

\subsection{Identification of a forwarding EI}

In a forwarding interaction, a server task forwards the request of a client to an entry in another server task and that entry, in turn, to another entry in a different server task, and so forth until the request is ultimately replied to the originating entry. The requests of the client tasks to the servers tasks can be represented by a number of cascaded segments, called the "multiple-segment" here. The head and end events of a multi-segment are the head event of the first segment and the end event of the last segment in this stack. Figure 5-23 shows a multiple segment consisting of three stacked segments.

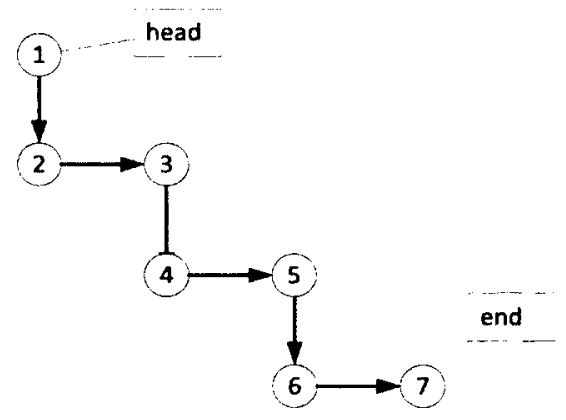

Figure 5-23 a multi-segment 
The identification of a forwarding EI is the same as that of a normal EI with one single difference: the initiating segment of a forwarding EI is a multi-segment. Figure 5-24 shows the initiating multi-segment in the anti-parallel, ordered and matched segment pair, which constitutes a forwarding EI.
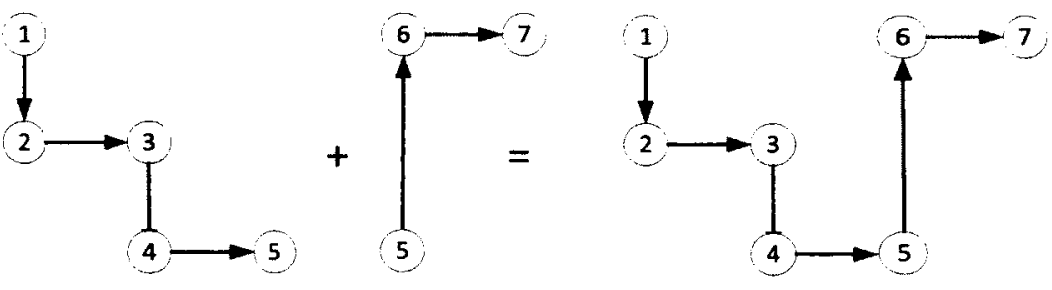

Figure 5-24 construction of a forwarding EI from an initiating multi-segment and an acknowledging single-segment

\subsection{Identification of the phase of an EI}

The relative position of EIs in an execution graph can be used to identify the phase of those EIs. For example, in the left side of Figure 5-25, EI1 is of phase one since it has occurred between the two segments of EI2. In this case the first event of EI1 is the end event of the IT eg_connector of EI2. In the right side of Figure 5-25, EI1 is of phase two since it has occurred right after EI2, such that the first event of EI1 is the end event of the SP eg_connector of EI2. 


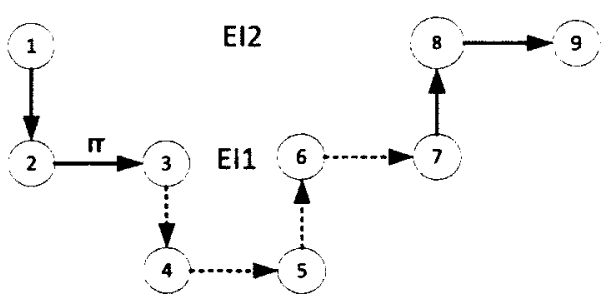

First phase

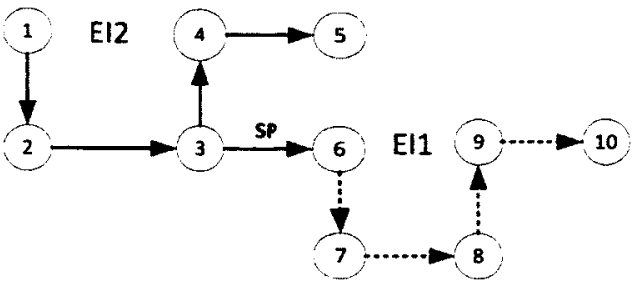

Second phase

Figure 5-25 event patterns of a first and a second phase EIs

The phase of the EIs in an EI identification process can be determined by analyzing the eg_connectors of the eg_connector-vector. If the type of this eg_connector is IT, it means that EI has happened between the two segments of another EI, therefore it is of phase one. In case the connecter is of type SP, it means that EI is in the second phase position of another EI, therefore it is of second phase.

The algorithm of Figure 5-26 can be used to set the phase of the identified EIs of an execution graph. This algorithm walks through the eg_connectors of the eg_connectorvector and, according to the type of each eg_connector, sets the phase of its EI.

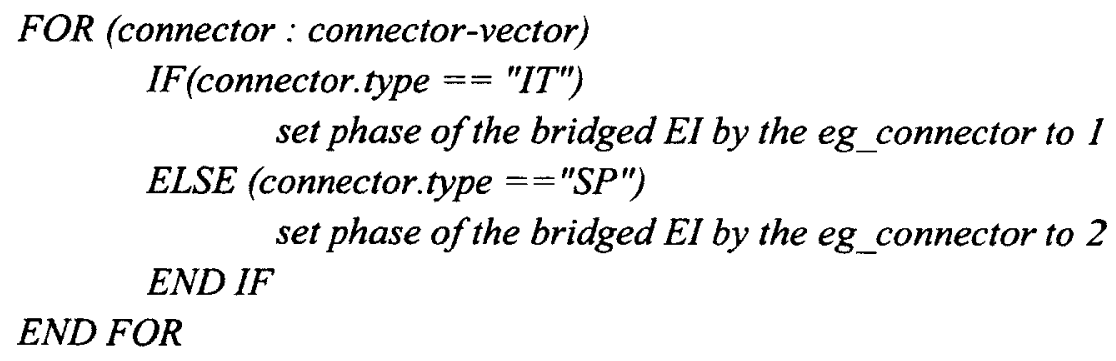

Figure 5-26 finding the phase in which an interaction has occurred 


\subsection{Summary}

This chapter illustrated how an execution graph can be transformed into vectors of EIs, tasks, entries and CPUs which have a closer semantic relationship with an LQN model from that of the execution graph. In the first step, the execution graph was first transformed into a vector of eg_threads. These eg_threads were then transformed into eg_connectors and eg_transitions. The eg_connectors and eg_transitions were then joined together to form segments. By matching up ordered and anti-parallel segments, the single-EIs were created and then joined together to form clustered-EIs or EIs. These EIs were then replaced with their bridging eg_connectors to allow for matching the remaining segments. The loop of segment to EI transformation, which is shown by the fat arrows between steps 4 and 5 in Figure 5-1, is iterated one less than the number of the layers of the computation to allow for collapse of all the segments and transformation of them into EIs. The thinner arrows between step 2 and 5 in Figure 5-1 indicate that the bridging eg_connectors are merged into the existing eg_connectors. The resulting EIs are highly enriched objects from which the entry part of an LQN model will be constructed. This subject is covered in the following chapter. 


\section{Chapter 6: Tasks Domain - LQN model construction}

The CPU, task, entry and EI vectors constructed in the previous chapter contain all the required information to construct an LQN model, this concept is shown in the diagram of Figure 6-1. However the attributes of the elements of some of these vectors are not at the level which they can be directly used in an LQN model and require some processing.

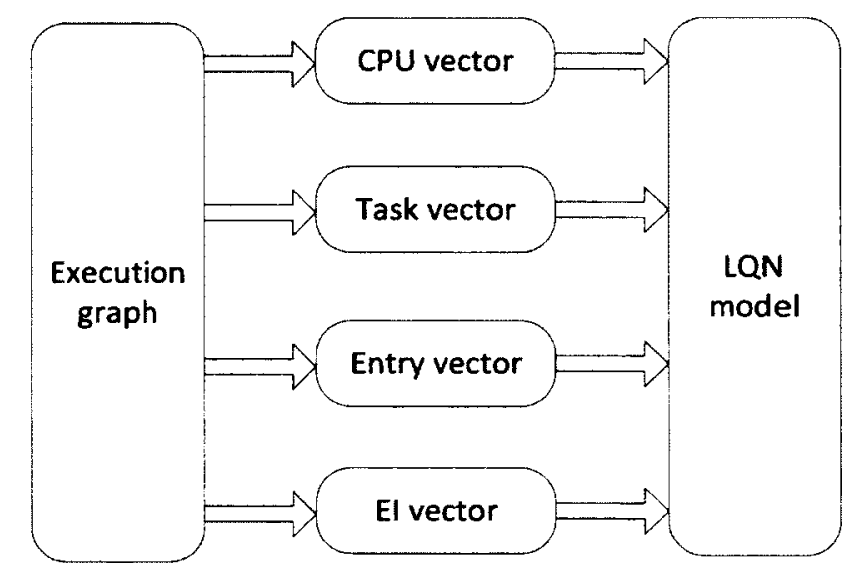

Figure 6-1 construction of an LQN model from CPU, task, entry and EI vectors

An LQN model consists of three parts, called the CPU, task and entry parts, which contain the parameters for the CPUs, tasks, and entries of a performance model, respectively. The CPU part contains parameters of the CPUs and includes the name and 
the multiplicity of the CPUs. The task part contains the parameters of the tasks which include the list of their entries, concurrency levels and the CPU on which these tasks execute. The entry part includes:

- The average CPU consumption times of the entries.

- The interaction between the entries.

An interaction between two entries is annotated with the ratio of the number of calls to the destination entry over the number of calls to the source entry. This ratio is called the "visit ratio".

The CPU and task vectors contain information from which the CPU and task parts of an LQN model can be easily constructed. However, the entry and EI vectors don't provide explicit data to be directly used in the entry part of an LQN model, so some additional processing of their contents is required. More specifically the EI vector can be used to:

- associate the entries of the entry vector with their CPU consumption times,

- create the TI objects which represent the interactions between the entries of the tasks, and

- annotate the TIs with their visit ratio numbers.

This chapter illustrates the analysis method used to process the EIs in order to construct the entry part of the LQN model.

The EIs of a computation represent the instances of interactions between the tasks of a computation. In order to create the interactions of an LQN model, these EIs need to be abstracted to a higher level called the TIs. A TI provides information such as the 
interacting tasks and entries, the type and the visit ratios of these interactions. This information is collected by processing each EI and integrating its parameters into those of its associated TI as well as those of the entries of that TI. This process builds the entry part of an LQN model.

In the first section of this chapter, a TI is characterized based on the definition of an interaction in the LQN model. In the subsequent sections, the methods which are used to extract the attributes of the TIs from the EIs, and determine the CPU-times of entries of a computation, are explained.

Throughout this chapter event types are identified by their acronym's which were defined in Subsection 4.2.3 and is repeated here for convenience of reading.

Table 6-1 event types and their brief description

\begin{tabular}{|l|c|l|}
\hline Event type & $\begin{array}{l}\text { Abbreviated } \\
\text { name }\end{array}$ & description \\
\hline Send & $S E$ & "send" event by a requesting task \\
\hline Receive & $R E$ & "receive" event by a server task \\
\hline Send acknowledge & $S A$ & Reply event in a server task \\
\hline Fork send & $F S$ & A non-blocking service request event \\
\hline Fork acknowledge & $F A$ & Non-blocking reply event in a server task \\
\hline end & $E N$ & Ending event of a eg_thread \\
\hline activity & $A C$ & A CPU-time consuming event \\
\hline external & $E X$ & Starting event of an operation \\
\hline
\end{tabular}




\subsection{Characterization of a TI}

The attributes of a TI are shown in Table 6-2.

Table 6-2 attributes of a TI

\begin{tabular}{|c|l|}
\hline No & attribute \\
\hline 1 & Task ordered set (client, server, forwarding1, forwarding2, ...) \\
\hline 2 & Entry ordered set \\
\hline 3 & Type (Synchronous or asynchronous or forwarding) \\
\hline 4 & Count-vector at phase 1 \\
\hline 5 & Count-vector at phase 2 \\
\hline
\end{tabular}

1. The task ordered set specifies the tasks involved in a TI. The first task in this set is the service requesting task, the second one is the server task, the third task is the first forwarding one, the fourth task is the second forwarding one and so on.

2. The entry ordered set specifies the entries involved in this interaction. The role of each component of this set is the same as that of the task ordered set.

3. The type specifies the type of an interaction which includes "Synchronous", "Asynchronous" and "Forwarding". In a Synchronous interaction the number of components of the task ordered set is two but, in the case of a Forwarding interaction type, this value is three or more.

4. The count vector can be used to determine the number of calls made from the client task to the other tasks of a TI. Each component of this vector represents the 
number of single-EIs of the clustered EIs which are mapped to this TI. For each phase, a separate count vector is specified.

The number of calls of a clustered EI is taken from the number of component of its segment-set vector (Sub-section 5.7.1).

\subsubsection{Visit ratio}

The visit ratio of the interaction between entry A and entry B is defined by the average number of calls to entry $B$ from entry A per interaction session. The visit ratio of an interaction is represented by an ordered set of two numbers for the visit ratios of the first and the second phases, respectively.

The count vectors of a TI and the incoming call numbers of the client entry of that TI can be used to determine that TI's visit ratio numbers. By summing up the elements of the count vector, one can determine the total number of the calls in each TI for the corresponding phase. Equation 6-1 shows that the visit ratio of a TI can be calculated from its attributes. The total number of calls of each TI at a particular phase will be divided by the same phase incoming calls of the TI's source entry and returned as the visit ratio of the interaction at that phase.

visit ratio of a TI at phase $i$

$$
=\frac{\sum \text { components of the phase } i \text { count vector of } T I}{\text { number of phase } i \text { incoming calls of the client entry of } T I}
$$




\subsection{EI mapping}

Each EI of an execution graph will contribute in the determination of the CPU consumption time of the entries and also the full characterization of the interactions between the entries of one particular TI. In fact each EI is an instance of one particular TI in the events domain. Obviously, there may be more than one EI that represents the same $\mathrm{TI}$ in the events domain. Therefore, the characterization of a TI is dependent upon the EIs which are mapped to that TI.

The classification of EIs to construct the TIs of an execution graph is in terms of:

1. The sets of task-entries in the source, destination and, in case of a forwarding interaction, the forwarding positions.

2. The type of EIs

It should be noted that the phase of EIs don't affect this classification process. When EIs are classified into a TI object, the phases of the mapped EIs are used for an internal sub-classification of the specifications of the EIs which are mapped to that TI object. In fact these specifications contribute to enhance different parameters of that TI. More accurately, the phase of an EI specifies to which count-vector (phase 1 or 2) the single-EI count of that EI will be added. The phase of EIs also determines the proper assignment of the CPU-times of those EIs to the CPU-time vector of their associated entries. This will be discussed further in Section 6.3. The algorithm of mapping an EI to a TI is shown in Figure 6-2. 
FOR (EI : EI_vector)

Identify tasks and entries of the source and destination events of $E I$

IF ( $a$ TI with the same set of tasks and entries AND same type exists)

myTI = the existing $T I$

ELSE

myTI $=$ create $a$ new $T I$

END IF

IF (the phase of EI is one)

- IF(the source task of myTI is of type "EX")

- Add "I" to the first phase incoming call vector of source entry of myTI END IF

- add "single-EI count" of EI to the phasel count-vector of myTI

- add " single-EI count " of EI to the "number of incoming calls at phase 1 " of the destination entry of myTI

- add the elements of the upper CPU-time vector of EI to the phase 1 CPU-time vector of the source entry of myTI

- add the elements of the lower CPU-time vector of EI to the phase ICPU-time vector of the destination entry of myTI

ELSE IF (the phase of EI is two)

- add " single-EI count " of EI to the phase2 count-vector of myTI

- add " 1 " to the "number of incoming calls at phase 2" of the source entry of myTI

- add" single-EI count " of EI to the "number of incoming calls at phase 1 " of the destination entry of myTI

- add elements of the upper CPU-time vector of EI to the phase 2 CPU-time vector of the source entry of myTI

- add elements of the lower CPU-time vector of EI to the phase 2 CPU-time vector of the destination entry of myTI

END IF

END FOR

Figure 6-2 mapping EIs to TIs and entries 
This algorithm examines the EIs of the EI vector and adds each EI's parameters to a different set of vectors based on the EI's phase. For example, when an EI of phase two is mapped to a TI:

- The EI's single-EI count is added to the phase two vector count of the TI

- The EI's single-EI count is added to the phase one incoming call number of its destination entry

- The elements of the upper CPU-time vector of EI is added to the phase two CPU-time vector of the source entry of TI

- The elements of the upper CPU-time vector of EI is added to the phase two CPU-time vector of the destination entry of TI

The first two items above are used to determine the visit ratios of the TI. As was mentioned before a visit ratio parameter can be determined by the number of calls in a TI and also the number of calls made to the source entry of that TI. When all the EIs which are associated with a TI add their single-EI count into the count vector of that $\mathrm{TI}$, in an appropriate phase, the number of calls of that TI will be determined. For determining the number of incoming calls made to an entry, one has to note that each call of a TI is in fact a call to the TI's destination entry. Therefore, the single-EI count of each mapped EI to a $\mathrm{TI}$ is also added to the incoming number of the destination entry of that $\mathrm{TI}$.

A call to an entry A from entry B, regardless of the phase in which the call has occurred, will only affect the first phase incoming call number of entry A. A call for a second phase operation of an entry will only be internally initiated by that entry itself. Therefore even when an EI of phase 2 is mapped to a TI: 
- The EI's single-EI count will be added to the phase one incoming call number of its destination entry, instead of that of phase 2 .

- The second phase incoming call number of that entry will be incremented by a value of 1 .

From a different perspective the update of the phase one incoming call number of an entry will occur in either of the following two circumstances:

- The entry is the destination entry of an interaction

- The entry belongs to the reference task of an execution graph

Therefore when an EI of first phase is mapped to a TI:

- If the source task of the EI is a reference task, a value of " 1 " will be added to the first phase incoming number of the source entry of the TI;

- The single-EI count of the EI will be added to incoming vector of the destination entry of the TI.

Figure 6-3 shows mapping of various EIs to their corresponding TI.

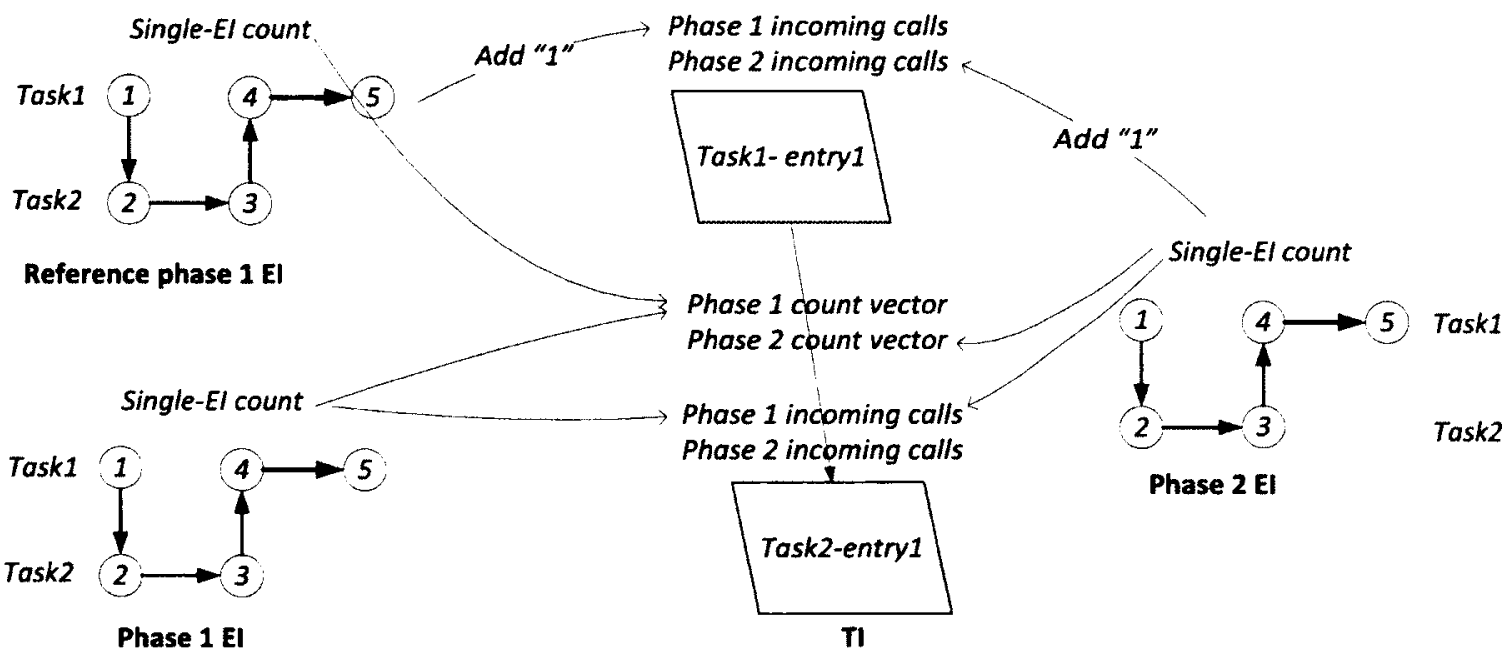

Figure 6-3 mapping of reference, phase 1 and phase 2 EIs to their corresponding TI 


\subsection{CPU-time}

The CPU-time of an entry specifies the average CPU consumption time of that entry in an invocation [56]. Determination of the CPU-time of an entry in is straightforward. First the CPU-times of all the execution events are processed and added into processing times of calls and transferred into the CPU-time vectors of an entry. Then, the CPU-time vectors of the entry are used to determine its CPU consumption times in its two phases of operations. This process is performed in several steps which are illustrated in the following sub-sections.

\subsubsection{Forming CPU-time vector of an eg_connector}

CPU-time of individual events are extracted from event-traces and added to an eg_connector's CPU-time vector. The algorithm of this operation, which is shown in Figure 6-4, starts from the "head" event of an eg_connector and adds its CPU-time into the eg_connector's CPU-time vector. It then proceeds to the next event in the execution graph and checks whether it is the end event of the eg_connector to end the process, or otherwise, adds its CPU-time into the vector again.

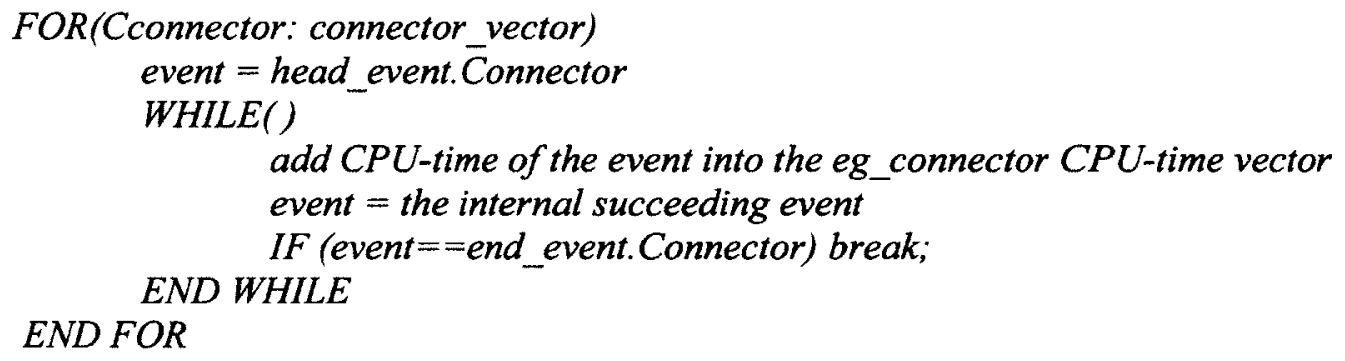

Figure 6-4 algorithm of forming CPU-time vector of connectors of an execution graph 


\subsubsection{CPU-time vector of a segment}

As explained in the previous chapter the elements of the CPU-time vector of eg_connectors of types "NB" or "AT" are mapped into the upper CPU-time vector of a segment and those of types "IT" and "NB" are into the lower CPU-time vector of the segment. The mapping occurs when a segment is created.

\subsubsection{CPU-time vector of an EI}

When a new single EI is created, the sum of the elements of each of the upper and lower CPU-time vector of each of its two constituent segments are added to the corresponding upper and lower, vectors of the new single EI, as shown in Figure 6-5. Therefore, each of these vectors in a single EI has two elements.

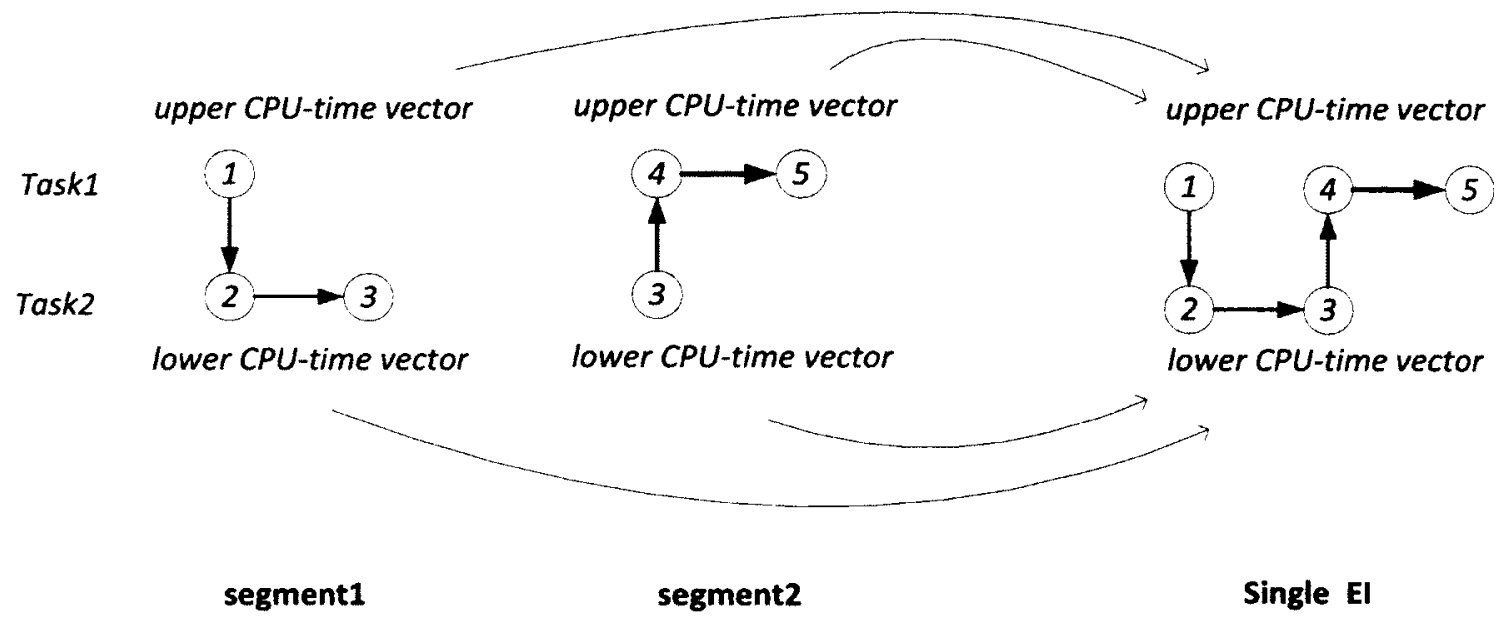

Figure 6-5 mapping CPU-times of two matching segments into their corresponding EI

When a single-EI is merged into an existing EI, the two elements of each of its CPUtime vectors (upper and lower) will be added to the corresponding (upper and lower, 
respectively) CPU-time vectors of the EI. Therefore the number of elements of each of these vectors in an EI represents twice the number of calls made by this EI.

\subsubsection{CPU-time vector of an entry}

As explained in the previous chapter, an entry has two vectors of CPU-times, one for each phase of operation. Elements of these vectors are provided by the upper and lower vectors of the EIs mapped to a TI. This mapping is based on the phase of the EI. The upper or lower vector of an EI determines whether an element is mapped to the source or destination entry of a TI and the phase determines to which vector of an entry it will be mapped. At the end of this process the phase 1 and 2 CPU-time vectors of the entries can be used to compute the phase 1 and 2 CPU-times of an entry, respectively.

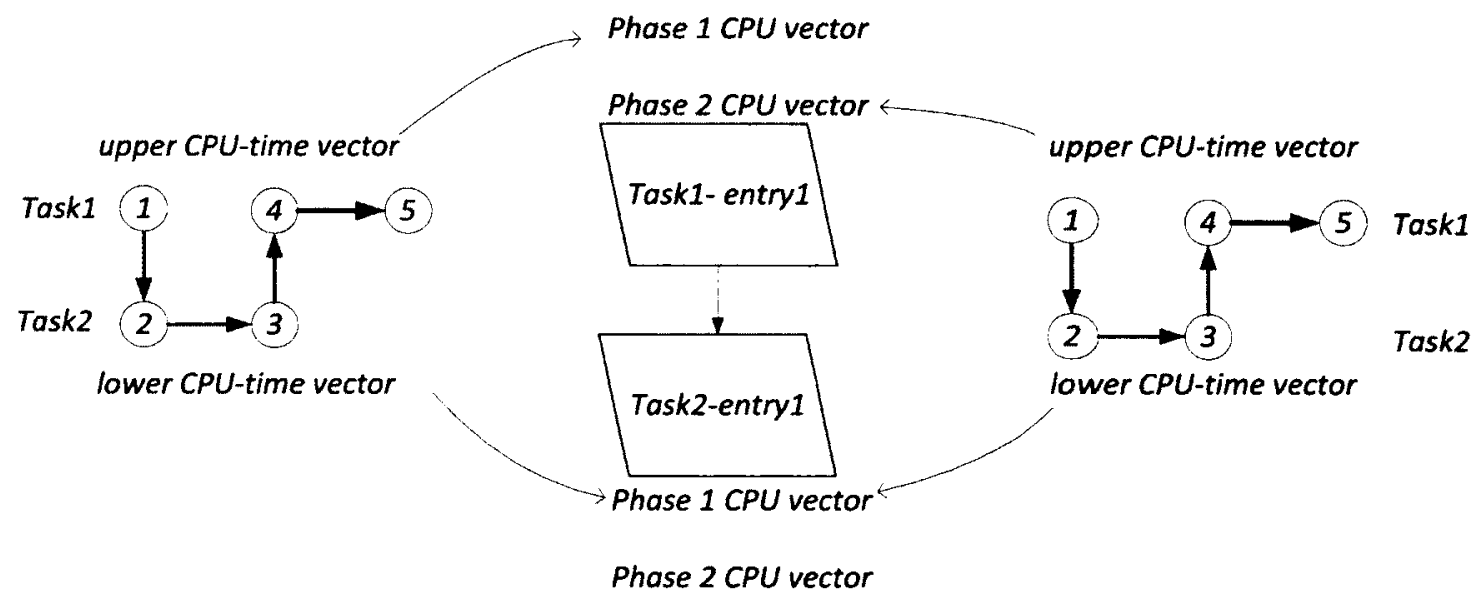

Phase $1 \mathrm{EI}$

TI

Phase 2 EI

Figure 6-6 mapping CPU-times of phase 1 and phase 2 type EIs into the entries of their corresponding $\mathbf{T I}$ 


\subsubsection{Determination of the CPU-times of an entry}

The phase 1 and phase 2 CPU-time vectors of an entry are used to determine the entry's CPU-times. The elements of each of these vectors represent the CPU-time consumption of a segment, which is one half of a call made or received by that entry. In other words, the number of elements in each of these vectors represents twice the number of calls in which an entry was involved. Therefore, to determine the average CPU-time of an entry, in each of its operation phase, the sum of the elements of the corresponding vector will be divided by half number of elements in the vector.

\subsection{Computational complexity of WebTime}

In the construction of the execution graph, each event in the vector of events is compared with all of its following events in the vector. That is the first event is compared with the following $n-1$ one, the second with that of $n-2$ and so on. Since, on average, a match is found half way through the comparison, the average number of total comparison operations made between events to construct an execution graph is:

computational complexity of execution graph

$$
=0.5(n-1)+0.5(n-2)+\cdots+0.5=0.25 n(n-1) \approx 0.25 n^{2}
$$

Equation 6-2

Where $n$ is the number of events. 
Finding the start events of the eg_threads has a linear computation complexity since it requires one scan of all the events.

Identification of the eg_connectors and formation of the eg_connector vector also has linear complexity since the next event in an eg_connector is found through that event's next successor attribute. Also if it can be assumed that in average each eg connector has 3 events, the average number of eg_connectors would be $n / 3$.

To construct the segments, each eg_connector will be tested against all the eg_transitions. Once a match is found, the matched eg_transition will not be involved in the remaining comparisons. Again, by assuming that a match is found half way through scanning the eg_transition vector, the computational complexity of constructing segments would be:

computational complexity of segments

$$
\begin{aligned}
& =0.5 n c+0.5(n c-1)+\cdots+0.5(n c-n t)=0.25 n c(n c-1) \\
& =0.25 \frac{n}{3}\left(\frac{n}{3}-1\right) \approx 0.028 n^{2}
\end{aligned}
$$

Equation 6-3

Where $n c$ is the number of eg_connectors and $n t$ is the number of eg transitions. It is also assumed that $n c=n t=\frac{n}{3}$

The single-EIs are constructed by testing one single-EI against the rest of them in the single-EI vector. A matched single-EI will not be involved in the remaining tests. By 
assuming that the average number of single-EIs is $\frac{n}{5}$, and, as was done for the segments, the computational complexity of creating single-EIs is:

$$
\begin{gathered}
\text { computational complexity of single }-E I s=0.5 n \operatorname{seg}+0.5(n s e g-1)+\cdots+0.5 \\
=0.25 n \operatorname{seg}(n \operatorname{seg}+1)=0.25 \frac{n}{5}\left(\frac{n}{5}+1\right) \approx 0.01 n^{2}
\end{gathered}
$$

Equation 6-4

Where nseg is the number of segments.

To construct EIs, each single-EI is tested against the rest in the single-EI vector. By assuming that the average number of events in a single-EI is 10 , the computational complexity of constructing EIs is:

$$
\begin{aligned}
& \text { computational complexity of constructiong EIs }=0.25 n \operatorname{sei}(n s e i+1) \\
& \qquad=0.25 \frac{n}{10}\left(\frac{n}{10}+1\right) \approx 0.0025 n^{2}
\end{aligned}
$$

Equation 6-5

Where nsei is the number of single-EIs.

The computational complexity of various processing steps of WebTime is summarized in Table 6-3. This table shows that WebTime has an overall quadratic computational complexity and a significant part of this complexity is associated with the construction of the execution graph. 
Table 6-3 computation complexity of various processes of WebTime

\begin{tabular}{|l|c|}
\hline Computational complexity & \\
\hline Execution graph & $0.25 n^{2}$ \\
\hline threads & $n$ \\
\hline connector & $n$ \\
\hline transition & $0.028 n^{2}$ \\
\hline segment & $0.001 n^{2}$ \\
\hline Single-EI & $0.0025 n^{2}$ \\
\hline EI & $0.28 n^{2}+3 n$ \\
\hline total & \\
\hline
\end{tabular}

\subsection{Scalability}

There are two different scenarios for scaling a system:

1. More resources may be added to a system to increase its capacity for a higher number of users for the same services.

2. The system is expanded for providing different services which, on average, requires more computation per an invocation of the system.

By considering the fact that construction of a sub-model has quadratic complexity, it can be concluded that the transformation performed by WebTime scales well for the first scenario but it doesn't scale well for the second. However an events log generated from a large number of invocations of a system with a large variety of services will tend to exhibit an "average behaviour" and therefore the analysis of this events log will still scale well. 


\subsection{Computation granularity}

In an attempt to make WebTime a scalable method and by considering the fact that the construction of a model from an execution graph has a quadratic computational complexity, it was decided to use a finer grain of processing than processing the whole events $\log$ of a computation. This means that instead of constructing a model for the whole events $\log$ of a computation, the events $\log$ will be broken down into a number of smaller events logs and a sub-model will be constructed for each of the smaller events logs. These sub-models will be then combined together in a linear fashion to construct the final LQN model. This reduces the computation load of the sub-model constructing process, which has a quadratic computational complexity, and instead combines multiple sub-models, which has a linear computational complexity. The following equation shows the computational complexity of constructing sub-models:

$$
\begin{aligned}
& \text { computational complexity of constructing sub_models } \\
& =\left(\text { number of invocations)(average number of events of invocations) }{ }^{2}\right.
\end{aligned}
$$

Equation 6-6

WebTime classifies the events in a large events log file based on the invocation in which they were involved and puts the events of each event class into a separate file called the invocation log file. An application may consist of several invocations of a system. When the number of invocations of a system under test increases, the size of the events $\log$ of that system will be larger but it doesn't necessarily change the average size 
of an invocation log for that system. WebTime creates an execution graph for each individual invocation and creates a separate sub-model for that execution graph.

WebTime performs a pre-processing of the events log of a computation to create the invocation $\log$ files for that computation. The events $\log$ of a computation consists of the events $\log$ of the individual tasks of that computation, which are generated in the nodes in which the tasks were executing. A task's events log consists of the event-traces of all the invocations in which that task was involved. The fact that an event-trace is annotated with the ID of the invocation in which the event occurred, allows WebTime to transform all the task events logs into invocation events logs. This transformation is performed by moving the events of events log of tasks to the appropriate invocation log file, according to their invocation id, as shown in Figure 6-7.

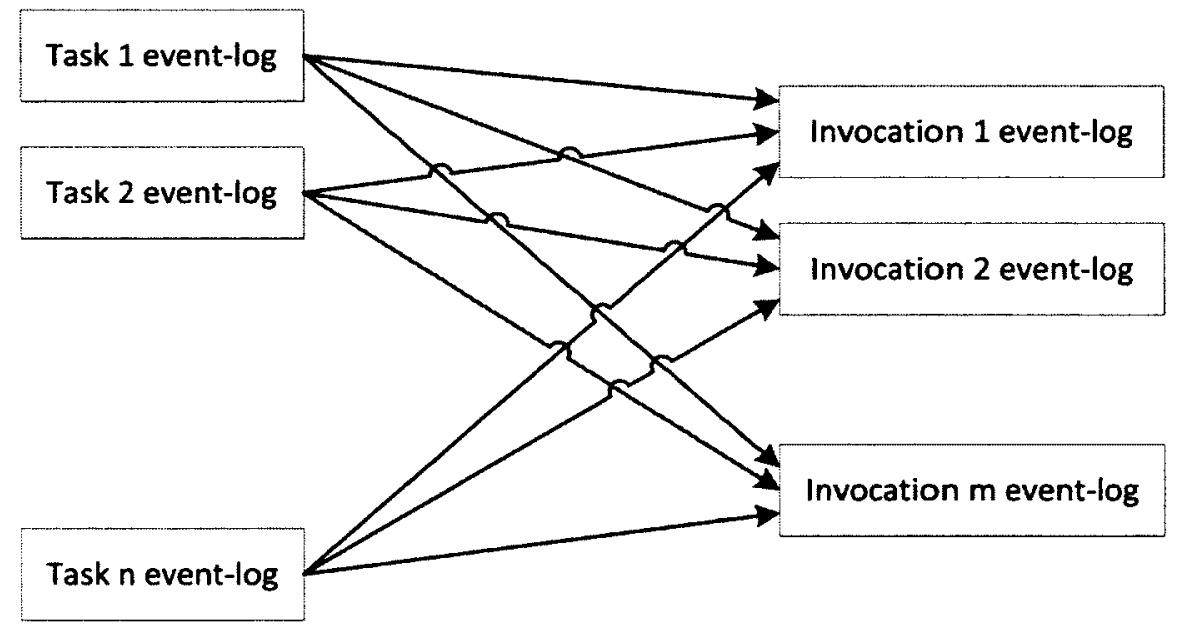

Figure 6-7 pre-processing of task events logs 


\subsection{Constructing a model from sub-models}

WebTime constructs the final LQN model of a computation by combining the submodels of a computation. The combining process consists of recovering the tasks, the CPU-times of entries and also the interaction between the entries of the system from the sub-models, combining these entities and using them to construct the final model, as shown in Figure 6-8. For this process, the system parses each sub-model, identifies tasks, entries and CPUs and creates an object for each of them. These objects will be put in their corresponding task, entry, $\mathrm{CPU}$ and interaction vectors. As the system parses through the sub-models, it either creates new objects or enhances the existing ones with the new parsed data. At the end, when all the sub-models are parsed, the system uses these vectors to construct the final LQN model, the same way that was done for constructing a submodel from these vectors which were extracted from an execution graph.

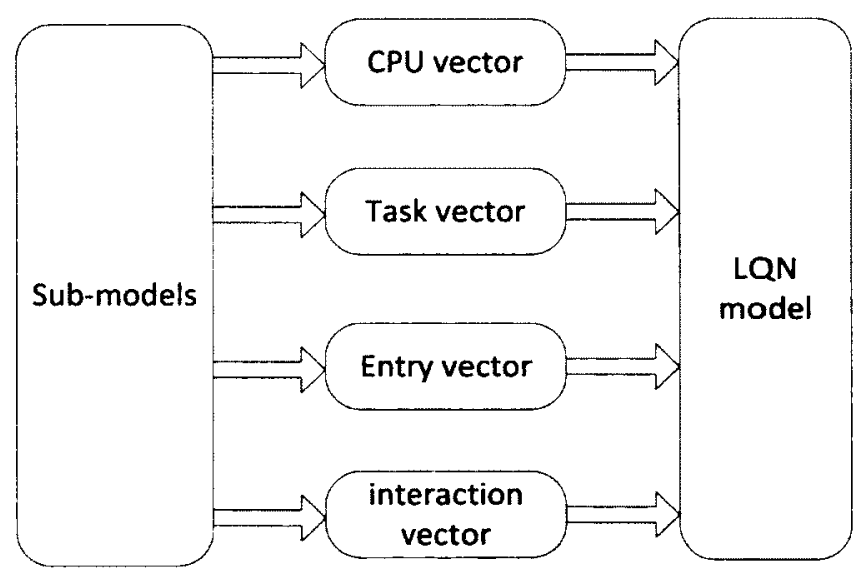

Figure 6-8 construction of the final LQN model of sub-models 
The attributes by which the entities such as tasks, CPUs, entries and interactions are characterized are shown in Table 6-4. These attributes are a limited version of those which were used in the previous chapter and will not explained here.

Table 6-4 attributes of tasks, CPUs, entries and interaction

\begin{tabular}{|c|c|c|c|c|}
\hline No & $\begin{array}{l}\text { Attributes of an } \\
\text { interaction }\end{array}$ & Attributes of a task & $\begin{array}{c}\text { Attributes of a } \\
\text { CPU }\end{array}$ & $\begin{array}{c}\text { Attributes of an } \\
\text { entry }\end{array}$ \\
\hline 1 & Entry name 1 & name & name & name \\
\hline 2 & Entry name 2 & reference & multiplicity & $\begin{array}{c}\text { Phase one } \\
\text { CPU-time vector }\end{array}$ \\
\hline 3 & $\begin{array}{c}\text { Phase one } \\
\text { visit-ratio vector }\end{array}$ & $\begin{array}{l}\text { Vector of entry } \\
\text { name }\end{array}$ & Scheduling policy & $\begin{array}{c}\text { Phase two } \\
\text { CPU-time vector }\end{array}$ \\
\hline 4 & $\begin{array}{c}\text { Phase two } \\
\text { visit-ratio vector }\end{array}$ & CPU name & & \\
\hline 5 & & multiplicity & & \\
\hline
\end{tabular}

The parser algorithm takes one sub-models at a time, scans each lines of that submodel, extracts that line's data, creates or finds the appropriate object for that data and updates that object. Figure 6-9 shows the parser algorithm.

After all the sub-models are parsed, the CPU, task, interaction and entry vectors will contain all the CPUs, tasks, entries and interactions of the computation, respectively. At this point the CPU and task vectors can be directly used to complete the CPU and the task parts of the final LQN model. The entry information for that model will be completed using the entry and interaction vectors. The visit ratio of an interaction at a particular phase is the average of all the components of the visit ratio vector of that interaction at 
that phase. The CPU-time of an entry is the average of all the components of the CPUtime vector of that entry at that phase.

\subsection{Model accuracy}

The accuracy of the model created by WebTime is dependent on several factors including:

- The extent by which the events of a computation are captured by the instrumentation system

- The accuracy by which the CPU consumption time of events are determined

- The chances of corruption of some of the logged event-traces due to fault in the monitoring system or the instrumentation system

The accuracy of the performance prediction of WebTime can be traded off with the required post-mortem processing power. The accuracy of the results depends upon the percentage of the events which are captured by the instrumentation system. However, a tight coverage of the events will lead to a higher volume of events log to be processed which will requires higher processing power or time. In other words, the required accuracy is a subjective parameter and depends upon the requirements of the application under test. For example, for the applications which require real time evaluation of performance with an acceptable accuracy level, the volume of events which should be monitored can be accommodated accordingly.

WebTime can tolerate errors in tracing and instrumentation. These errors will translate to fewer captured single-EIs. It should be noted that these single-EIs are mapped 
to their respective TIs in order to determine the service time values of the source and destination entries and the request rate values of these TI. Therefore a small percentage of less identified EIs will only compromise the accuracy of these values instead of compromising the whole model construction capability of WebTime.

\subsection{Summary}

This chapter has shown that the CPU and task vectors generated from an execution graph can be directly used to create the CPU and task parts of a model. The entry part of the model can be created using the data of the entry and EI vectors, which are extracted from the execution graph.

The construction of a model from an execution graph has a quadratic complexity. To reduce the share of quadratic computations in constructing a model, WebTime breaks a large events log into many invocation events logs and creates a sub-model for each of the invocation events log. These sub-models are then combined to construct the final model. 
FOR (sub-mode : sub-model vector)

Line $=$ Read one line from the sub-model

IF ( line is taken from the CPU part AND line starts with " $p$ ") / /CPU part

$p_{-} n=$ second word of the line

$I \bar{F}\left(C P U\right.$ vector doesn't contain a $C P U$ with the name of $\left.p \_n\right)$

$c p u=$ create a $C P U$ with the name of cpu AND put it in the CPU vector

cpu.name $=p \_n$

cpu.scheduling $=$ third word of the line

cpu.multiplicity $=$ fifth word of the line

END IF

ELSE IF (line is taken from the task part AND line starts with " $t$ ") //task part

$t_{-} n=$ second word of the line

$\overline{I F}$ (task vector doesn't contain a task with the name of $t_{-} n$ )

task $=$ get the task with the name of $t$ n from the task vector

WHILE ( $(n w=$ next word in the line $) !="-1 "$

task.entryVector.add ( $n w$ )

END WHILE

\section{ELSE IF}

task = create a new task

task.name $=t$ n

task.reference $=$ next word in the line

WHILE ( $(n w=$ next word in the line) $!="-l "$

task.entryVector.add (nw)

END WHILE

task.cpu $=$ next word in the line

task.multiplicity $=$ next word in the line

END IF

ELSE IF (line is taken from the entry part AND line starts with s) Hentry part

$e_{-} n=$ second word of the line

entry = get the entry with $e_{-} n$ from the entry vector

entry.phase1_CPU_time = third word of the line

entry.phase2_CPU_time = forth word of the line

ELSE IF (line is taken from the entry part AND line starts with $y$ )

$e_{-} n l=$ second word of the line

$e_{-} n 2=$ third word of the line

$\overline{I F}$ (interaction vector contains an interaction between $e_{-} n 1$ and $e \_n 2$ )

interaction $=$ get the interaction between $e_{-} n l$ and $\bar{e}_{-} n 2$ from interaction vector ELSE

interaction $=$ create an interaction between $e_{-} n l$ and $e \_n 2$ AND

END IF

put it in the interaction vector

interaction.phasel_visit-ratio $=$ third word of the line

interaction.phase 2_visit-ratio $=$ forth word of the line

END IF

END FOR

Figure 6-9 parsing CPUs, tasks and entries data from a sub-model 


\section{Chapter 7: Construction of an activity graph model}

An activity graph is an extended version of a layered queuing network (LQN) model which allows modeling various parallelism techniques applied by software designers to improve the resulting performance of an application. Activity graphs are typically used to model the performance related aspects of a software system when a task has complex internal behaviour such as forks and joins, or to simplify the specification of the performance behaviour of an application. This chapter introduces a method to automate the process of activity graph construction of a distributed software application.

Throughout this chapter event types are identified by their acronym's which were defined in Subsection 4.2.3 and is repeated here for convenience of reading.

Table 7-1 event types and their brief description

\begin{tabular}{|l|c|l|}
\hline Event type & $\begin{array}{l}\text { Abbreviated } \\
\text { name }\end{array}$ & description \\
\hline Send & $S E$ & "send" event by a requesting task \\
\hline Receive & $R E$ & "receive" event by a server task \\
\hline Send acknowledge & $S A$ & Reply event in a server task \\
\hline Fork send & $F S$ & A non-blocking service request event \\
\hline Fork acknowledge & $F A$ & Non-blocking reply event in a server task \\
\hline end & $E N$ & Ending event of a eg_thread \\
\hline activity & $A C$ & A CPU-time consuming event \\
\hline external & $E X$ & Starting event of an operation \\
\hline
\end{tabular}




\subsection{Introduction}

Activity graphs can represent the behaviour of an entry with a more detailed description than the "phases" method described earlier (In fact a phase is a single activity, or two activities for two-phase servers, in an activity graph). Activity graphs allow modeling various forms of parallelism in software systems. Techniques such as asynchronous remote procedure calls, early replies [63] and concurrency within tasks are various ways for preventing resources bottlenecks and for improving performance. Accurate modeling of these techniques, despite their popularity, is a challenging task. These techniques are not supported by the queuing network analysis [4], even with hierarchical decomposition. Stochastic rendezvous network (SRVN) [61] and the method of layers (MOL) [59] can only model systems with early replies.

The activity graph construction method presented in this chapter can be applied on a class of software systems in which the tasks use internal fork or join activities only when interacting with other tasks. This method uses the same execution graph of which was defined in Chapter 4. This method inherits many good features of the "phases" method including the independence from the wall clock time which makes activity graphs suitable for modeling distributed applications.

\subsection{Activity Graph Model}

This section provides a short description of activity graphs and supports that by a number of examples. A comprehensive description of this method is provided in [64] and [65]. In an activity graph an activity is a basic unit of behaviour. Activities are linked 
together by a directed graph to represent the precedence, or causal relationship between events. When a request arrives at an entry in a task of a computing system, it triggers the first activity of the activity graph of that computation. Subsequent activities may follow sequentially, or may fork into multiple paths which later join. Activities, like entriescan make requests to other entries at lower layers. The nomenclature used to represent activities, adopted from [64] and [65], is shown in Table 7-2 and those of their connections are shown in Table 7-3.

Table 7-2 the nomenclatures to show activities

\begin{tabular}{|c|c|c|}
\hline Name & Icon & Description \\
\hline Activity & a & Basic unit of modeling detail \\
\hline Activity with reply & $a[e]$ & $\begin{array}{l}\text { And entry that generates reply to entry e after it } \\
\text { executes }\end{array}$ \\
\hline
\end{tabular}

Table 7-3 the nomenclatures to show the connection between activities

\begin{tabular}{|l|l|l|}
\hline Name & Icon & Description \\
\hline Connecting-arc & & Transfer of control \\
\hline And-fork & & Start of concurrent execution \\
\hline And-join & & \\
\hline Repetition & & A synchronization point for concurrent activities \\
\hline
\end{tabular}


The following sub-sections introduce the activity graph using examples of several types of interactions. This introduction helps to understand the subsequent sections which explain the process of automatic activity graph construction of a computation.

\subsubsection{Activity graph of a Synchronous interaction}

The left side of Figure 7-1 shows the execution graph of a Synchronous interaction whose activity graph is shown at the right side of this figure. Activity 1 of Task "t1" makes a request to Task "t2" and blocks for a reply before proceeding to activity 4 . Entry "el" takes the request, passes it to Activity 2 and that to Activity 3 which replies to Entry "e2". This entry then replies in turn to the request of Activity 1 by which the control is passed to Activities 4 and then 5 .

e1

e2

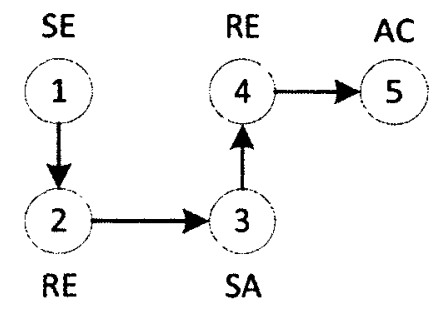

EI

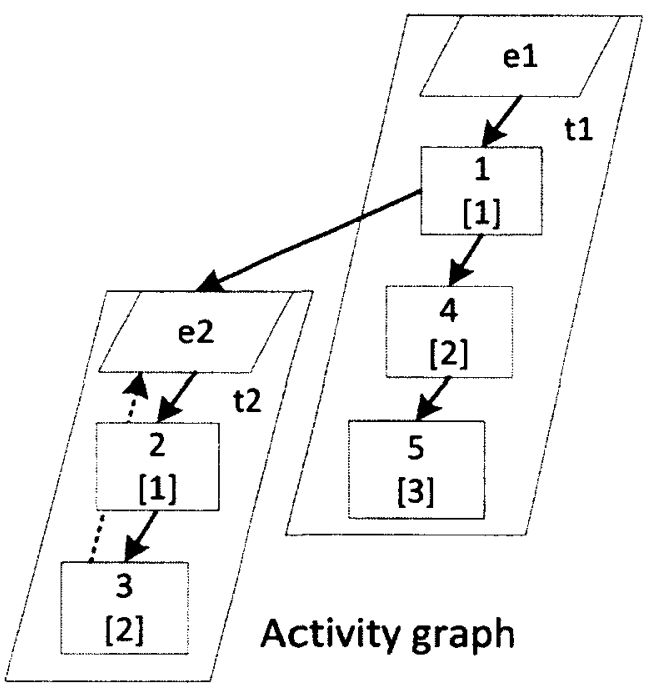

Activity graph

Figure 7-1 an RPC interaction and its activity graph 


\subsubsection{Activity graph of an asynchronous interaction}

The left side of Figure 7-2 shows the execution graph of an asynchronous interaction whose activity graph is shown at the right side of this figure. Event 2 initiates a request to Task 2 then passes control to Event 3 and then Event 4 and then blocks for a reply from Task2. This blocking situation is shown by "and-fork" of Event 1 to Events 2 and 3. The blocking situation is "and-join" of Events 4 and 2 to Event 5.

e1

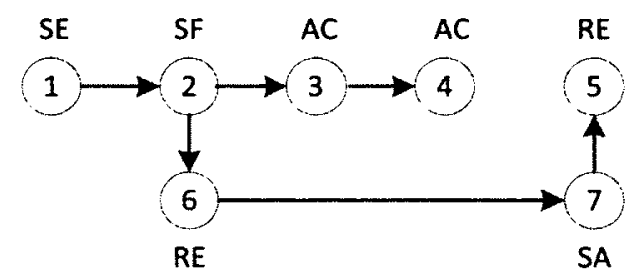

El

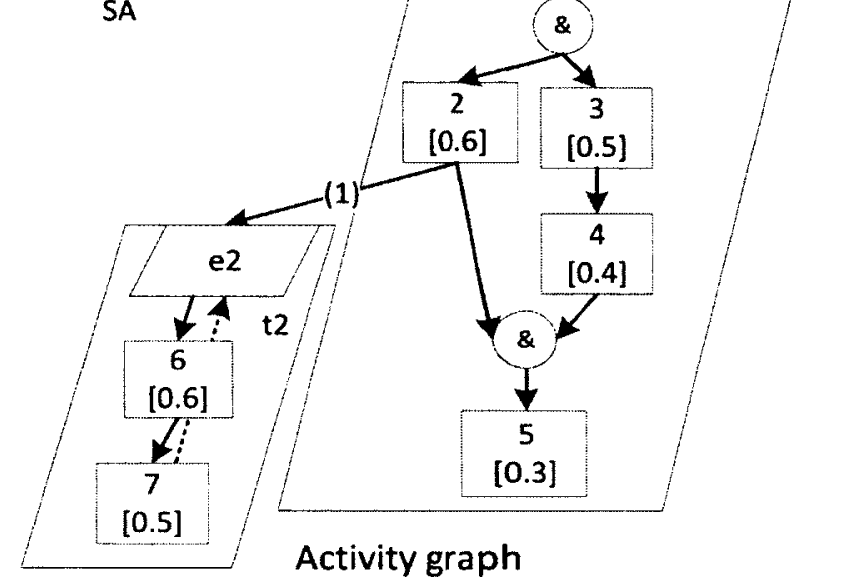

Figure 7-2 an asynchronous RPC interaction and its activity graph

\subsubsection{Activity graph of an interaction with second phase}

The right side of Figure 7-3 shows the execution graph of a Synchronous interaction with a second phase of operation whose activity graph is shown at the right side of this 
figure. In this case, Event 4 responds to Task "t1" as a result of a fork from Event 3. Event 5 concurrently takes the control and passes it to Event 6 where the second phase operation stops. This reply is modeled by an and-fork of Event 3 to Events 4 and 5 .

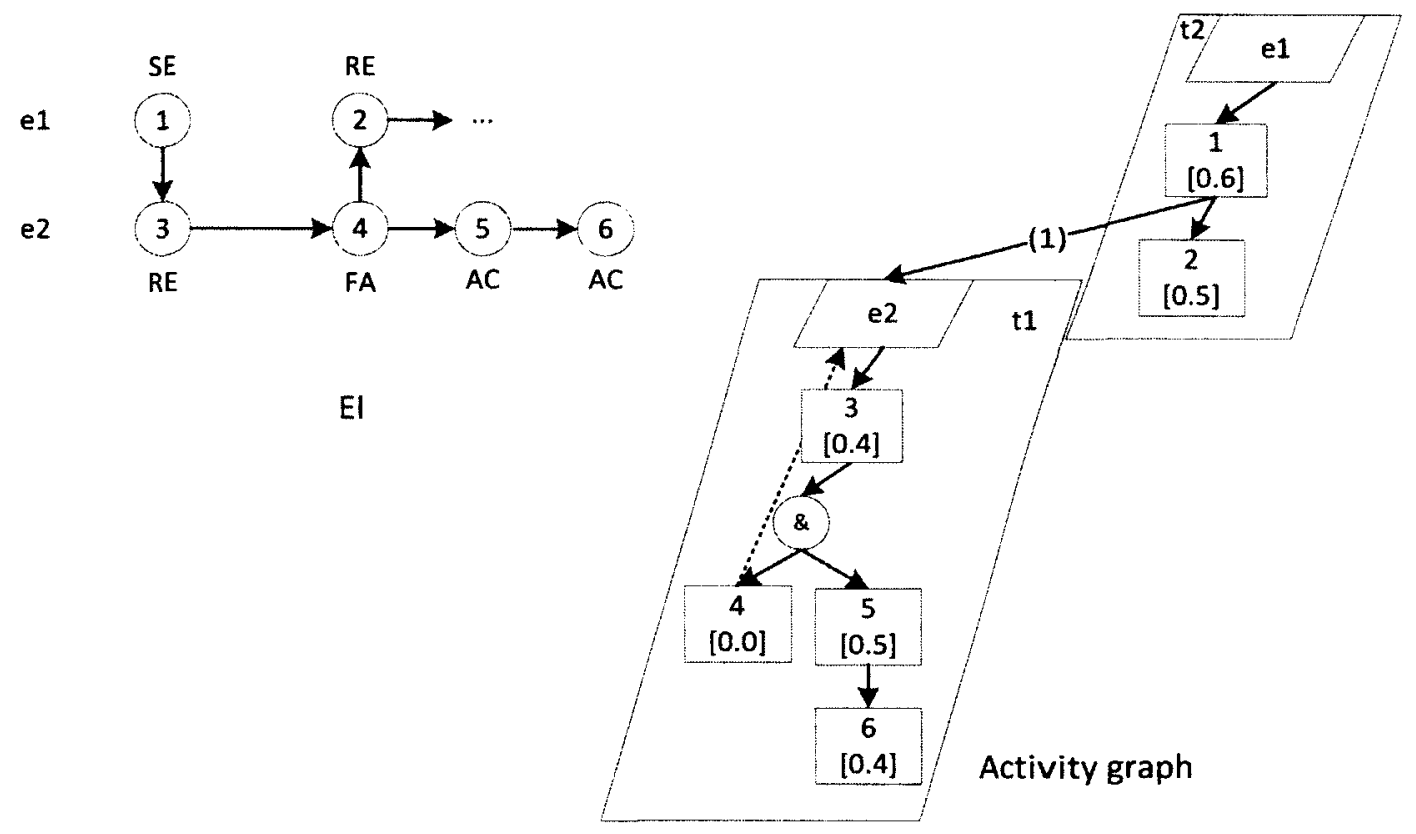

Figure 7-3 an RPC interaction with second phase and its activity graph

\subsection{Automatic Activity-Graph Construction Process}

In this work, activity graphs are constructed from eg_connectors of a computation. For each eg_connector of a task, an activity graph is constructed, called the "partial activity graph" or PAG of that eg_connector.

The approach to construct an activity graph of a computation consists of:

1. Identification of the eg_connectors of the computation, 
2. mapping the eg_connectors to the tasks in which they were executed,

3. selection of the representative eg_connectors of each task,

4. transformation of the representative eg_connectors into PAGs,

5. parameterization of the PAGs,

6. combining the PAGs into the final activity graph.

Figure 7-4 shows the detailed block diagram of the activity graph construction process. Blocks of 1 to 9 , and also 11, of this diagram show the process of EIs and TIs construction, which was illustrated in Chapters 4,5 and 6. The arrow between Blocks 4 and 9 specifies that the eg_connectors are mapped into their corresponding tasks where they are filtered to select the representative eg_connectors of those tasks. Block 10 specifies that the representative eg_connectors in each task are used to construct that task's PAG. The arrow between Blocks 11 and 10 specifies that the TIs of this computation are used to parameterize PAGs. The arrow between Block 10 and 12 specifies that the PAGs of all tasks are combined to construct the final activity graph.

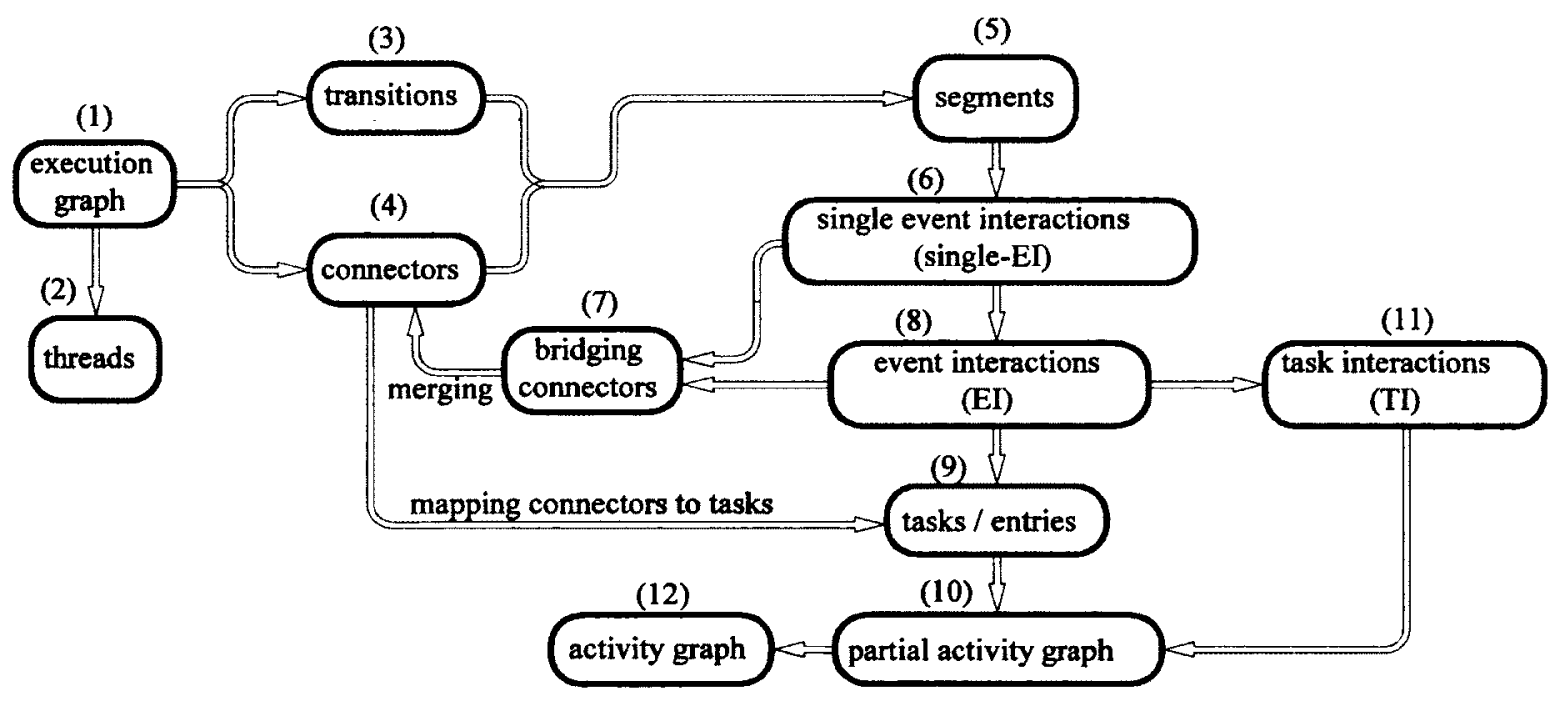

Figure 7-4 the activity graph construction overview diagram 


\subsection{Pre-processing of the Eg_connectors}

The eg_connectors of an execution graph are used to form the activity graphs of the tasks in that execution graph. However, using all eg_connectors provides redundant information about the activities of a task. Therefore, a pre-processing of the eg_connectors is used to identify the necessary eg_connectors which can represent the activities of a task and filter out the rest. This pre-processing is performed in a number steps which are explained in the following sub-sections.

\subsubsection{Classification}

Eg_connectors are classified into the tasks in which they have been executed. For this purpose, the task object of the transformer program contains another vector named the "task-eg_connector-vector". The IDs of the eg_connectors of a task are added to the taskeg_connector-vector of that task.

\subsubsection{Filtering}

The approach to reconstruct the architecture of a software application is to identify all the repeated patterns of events and select one instance as an architectural representative of each pattern. The representative pattern will then be used to identify the eg_connectors from which the activities of each task are specified. This implies that the eg_connectors that don't belong to any representative pattern should be filtered out.

A representative pattern in an execution graph is a single instance of a repeated pattern. For example, one EI from the EIs which are mapped to a TI, one single-EI from the clustered single-EIs of an EI, and finally the eg_connectors of the representative 
single-EIs. This description leads to the procedure by which the representative eg_connectors of the tasks of an execution graph can be identified, which is explained below.

\subsubsection{Filtering algorithm of task eg_connectors}

Figure 7-5 shows the algorithm for filtering the eg_connectors in the taskeg_connector-vector of a task. It starts by, identification of the first EI of the computation, which starts with an "EX" type eg_connector. This EI is used to confirm the eg_connectors in the task-eg_connector-vector of its source task, i.e. for filtering the rest out. The same operation will be performed on the EI nesting inside the first single EI of the previous EI to confirm the eg_connectors of the second task. This operation will progressively be performed until the interaction between the last two layers is reached. For the last layer, only the destination eg_connector of the first single EI will be confirmed.

identification of all clustered EIs

currentEI = selecting the EI which starts with "EX" connector

WHILEO

confirm all connectors of currentEI that belong to the origination task

IF (bridged EI by eg_connector "TI" of currentEI ==0) confirm all connectors of currentEI that belong to the destination task break

ENDIF

currentEI = bridged EI by eg_connector "TI" of currentEI

END WHILE

Figure 7-5 algorithm of filtering the connectors of a task 
Figure 7-6 shows a simple example of an execution graph which consists of one EI. This EI, in turn, contains two single-EIs. Obviously, only this EI can be selected as the representative EI of the execution graph. According to the algorithm, no filtering is required on the client's task-eg_connector-vector. For the server's task-eg_connectorvector, only eg_connector "c4" is confirmed. Figure 7-7 shows the activity graph of this computation. As can be seen, eg_connectors $\mathrm{c} 1, \mathrm{c} 2$ and $\mathrm{c} 3$ are used to construct the activity graph of the client task and only eg_connector $\mathrm{c} 4$ is used to construct the activity graph of the server task. For the server task, the precedence relation of only eg_connector $c 4$, as the representative of repeated patterns, will be written.

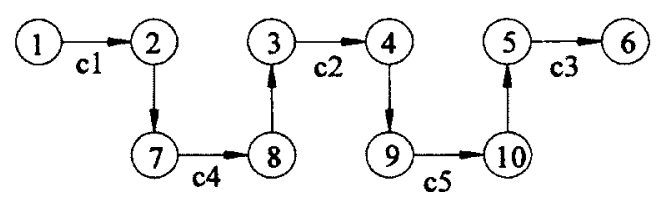

Figure 7-6 a clustered EI between two tasks

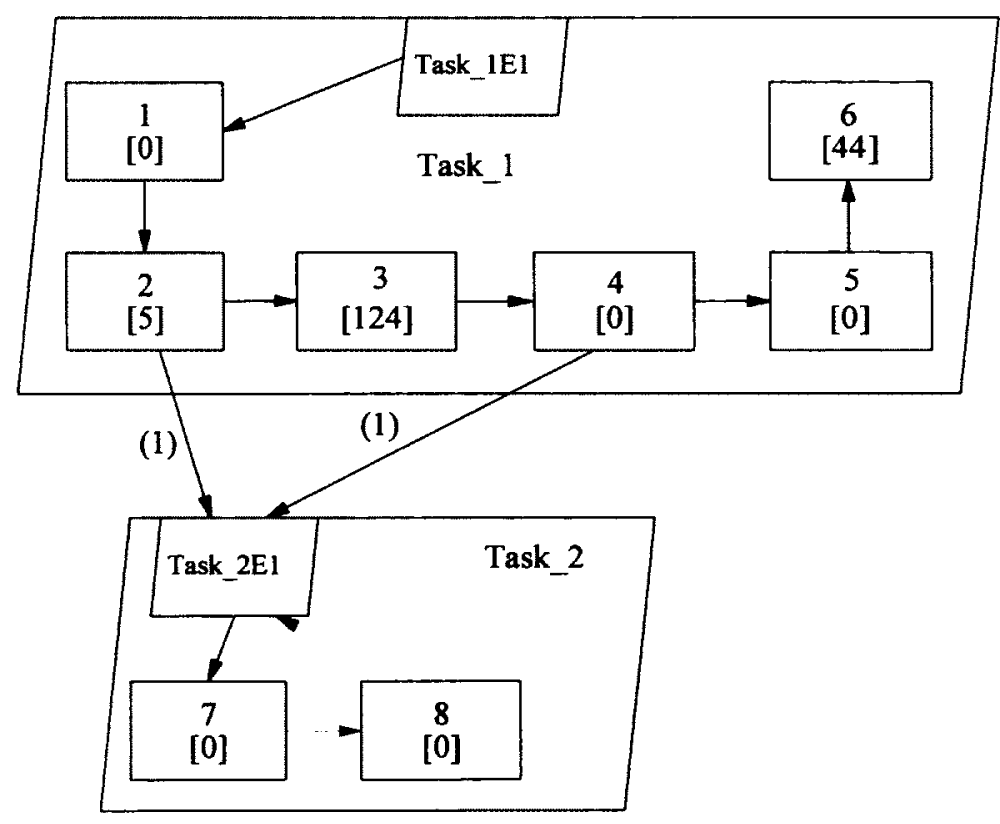

Figure 7-7 activity graph of the interaction in Figure 7-6 
Figure 7-8 shows another example of finding the representative eg_connectors of an execution graph. The upper part of the figure shows the execution graph and the lower part shows the confirmed eg_connectors of each task. There is one single-EI between Task_1and Task_2. Therefore, all eg_connectors of Task_1 are confirmed. The nested EI of this single-EI, which is the EI between Task_2 and Task_3, is composed of two single EIs. Therefore, all eg_connectors of Task_ 2 are confirmed. More ever, for Task_ 3 the EI nested in the first single-EI of the EI between Task_ 2 and Task_ 3 should be investigated. According to this, EI eg_connectors c10, c11, c12, c13 and c14 are confirmed. For Task_4, the eg_connectors of the first single-EI, which is eg_connector $c 20$, is confirmed.

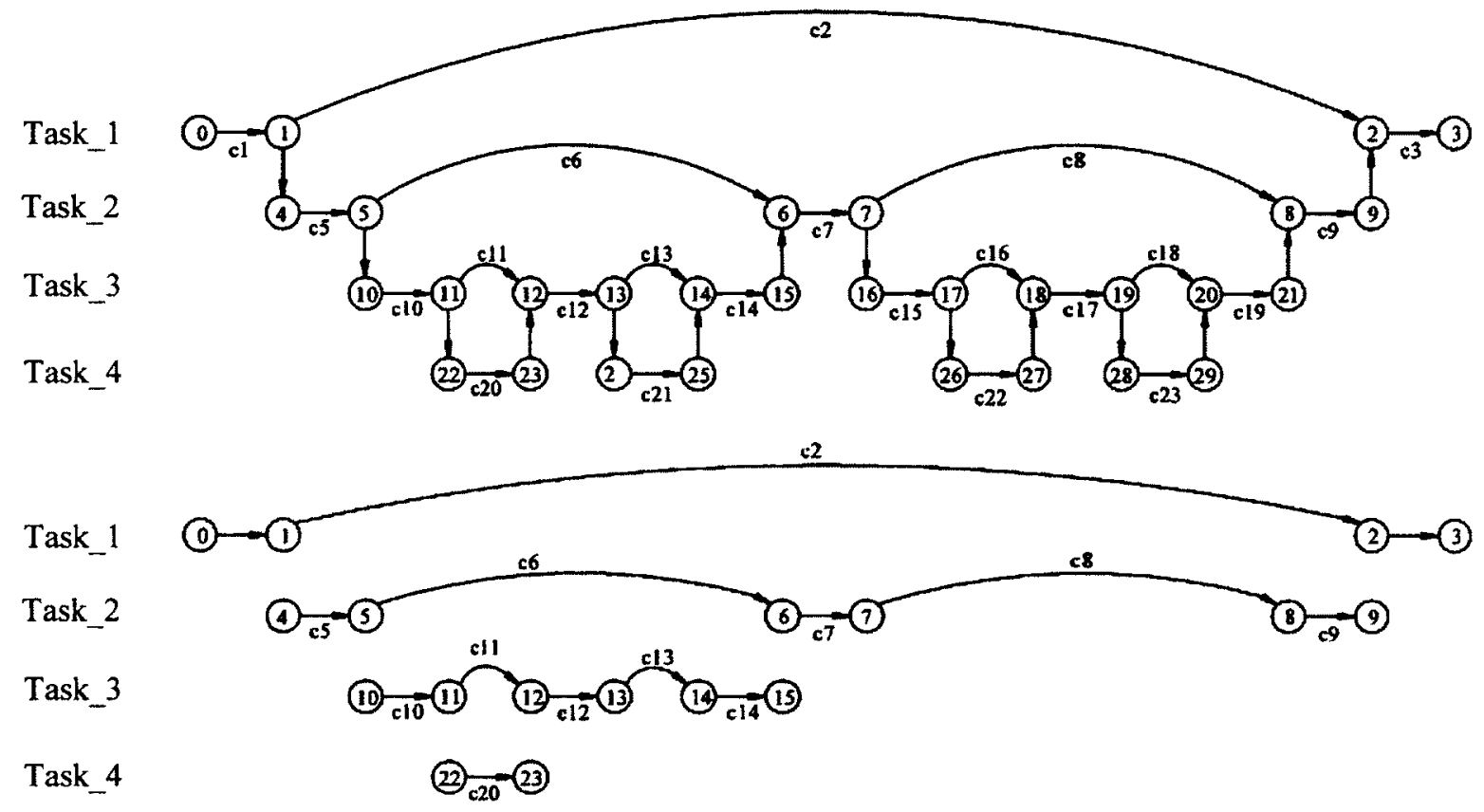

Figure 7-8 application of algorithm of filtering connectors of tasks 


\subsubsection{Ordering}

The filtered eg_connectors of a task must be ordered in order to construct its activity graph. The ordering process cannot proceed if the eg_connectors of a task are not linked in a continuous fashion. Unfortunately, there may be gaps between the eg_connectors of a task due to blocking interactions of these tasks. The method used here is to modify the eg_connector object, defined in Chapter 5 , in order that it can also be used to fill these gaps. This object is modified such that a bridging eg_connector can also bridge over single EIs, as opposed to only bridging over EIs. This modification is shown in Figure 7-9. The first part of this figure shows that the original bridging eg_connector cannot fill the gap between Eg_connectors $\mathrm{c3}$ and c6. The second part of this figure shows the modifications made on the object of an eg_connector. This object is equipped with a new pair of head and end events named the "inner-head" and the "inner-end", respectively. Now this eg_connector with its inner head and end can fill the gap between Events 4 and 7. This object is also equipped with an "extended end", which is its end when a bridging eg_connector is merged into it, as shown in third part of Figure 7-9. This eg_connector, when used in the EI identification process, uses its extended end but, when used for activity graph construction, uses its real end. 


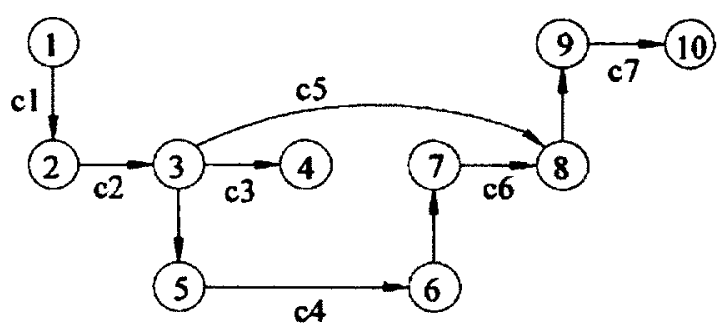

1. bridging a single El to identify the El in the higher layer

c5:

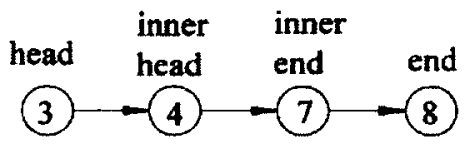

2. New structure of the bridging connector

c2:

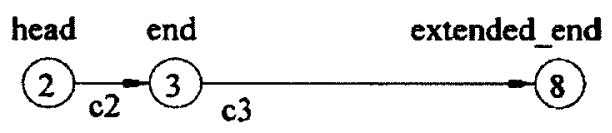

3. structure of a normal eg_connector when extended by a bridging connector

Figure 7-9 structures of a normal and a bridging connector

\subsection{Partial activity graph (PAG)}

When all the eg_connectors in a task are lined up, their continuity restored using bridging eg_connectors and the redundant ones filtered out, the process of activity graph construction may start. In this process a PAG is constructed for each eg_connector and then all the PAGs of a task are joined together, in the order in which their corresponding eg_connectors were executed in that task.

The construction of a PAG from an eg_connector consists of: 
1. Adding one activity for each event of the eg_connector, except the head event, in the PAG.

2. Transferring the $\mathrm{CPU}$ consumption time of the events into their corresponding activities.

3. Finding the type of precedence connection between activities.

Except for the activity of an end event, the activities of all other events of an eg_connector are simply connected through the "connecting arc" precedence connections, in the same order as that of the events of the eg_connector. The type of the end event, or the head event, determines the way in which the eg_connector interfaces with the succeeding or preceding eg_connector.

The process of PAG construction is end-event oriented, meaning that the activity interfaces are written for the end event of an eg_connector, instead of the head event of the following eg_connector. An activity is not added for the head event of an eg_connector in the PAG of that eg_connector, because, this activity is added to the PAG of the preceding eg_connector (these two eg_connectors share that event).

Table 7-4 summarizes the type of connections used interfacing PAGs based on the end or head event types of their eg_connector. As can be seen, except for one case, the interfaces are determined based on the type of end events. The way in which these interfaces are constructed is explained by example in the following sub-sections. 
Table 7-4 end interfaces of the PAGs of various connectors

\begin{tabular}{|c|c|l|l|}
\hline Head event & End event & Interfacing icon & \multicolumn{1}{|c|}{ comments } \\
\hline & SE & Repetition & Follows by a bridging connector \\
\hline & SA & And-fork & Connection to self-entry \\
\hline & FS & And-fork & Follows by a bridging connector \\
\hline & FA & And-fork & Connection to self-entry \\
\hline FS & EN & none & \\
\hline
\end{tabular}

\subsubsection{PAG for an eg_connector with end event of type SE}

The top side of Figure 7-10 shows an example of an eg_connector with the end event of type SE and the bottom side shows the PAG of this eg_connector (This eg_connector will be followed by a bridging eg_connector in the execution graph). The end event is shown by the "repetition" icon in the PAG which represents the blocking for the request sent to a server. This icon has a precedence connection with its following activity in this entry, shown by the "Next-con" (the first activity of the following eg_connector after a bridging eg_connector). The specification of this activity is not included in this PAG but the precedence connection to that is included (this is why this activity is shown by a different line pattern box). Transfer of control to that activity will be made after a response is received from the server to Activity 4 . 

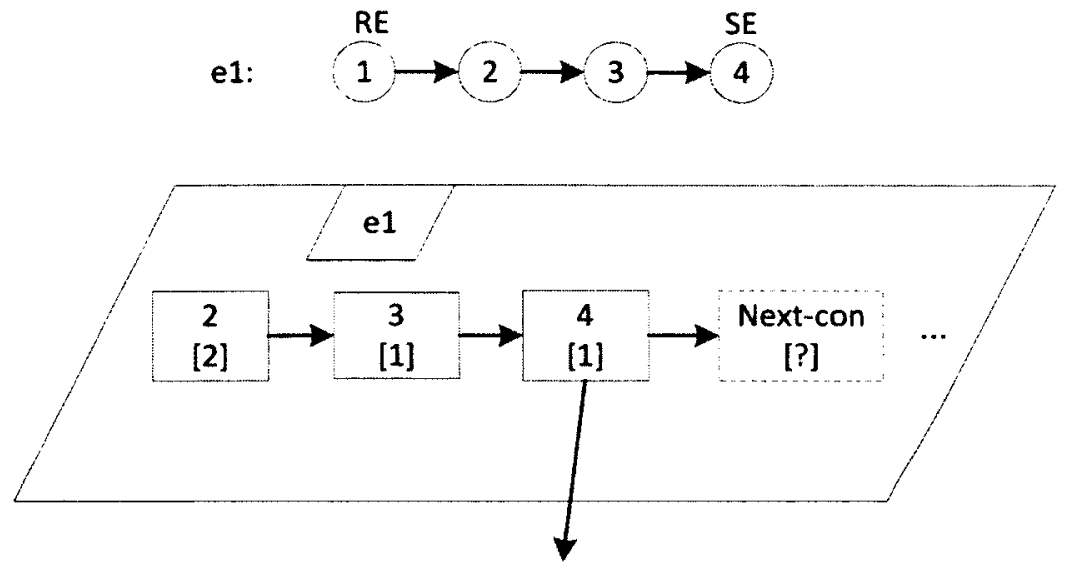

Figure 7-10 the PAG of an eg_connector with SE type end event

\subsubsection{PAG for an eg_connector with end event of type FA}

The top side of Figure 7-11 shows an example of an eg_connector with the end event of type FA and the bottom side shows the PAG of this eg_connector. The activity before the end activity of this TAG uses the and-fork connection to fork into the end activity of this TAG and the first activity of the following PAG (second event of the eg_connector of that PAG), shown here by the "next-con". The specification of this activity is not included in this PAG but a precedence connection to that is included (this is why this activity is shown by a different line pattern box). The end activity of this TAG responds to the entry of its TAG. 


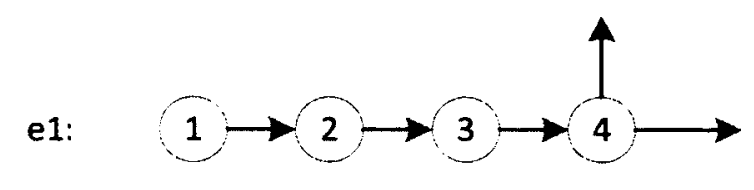

RE

FA

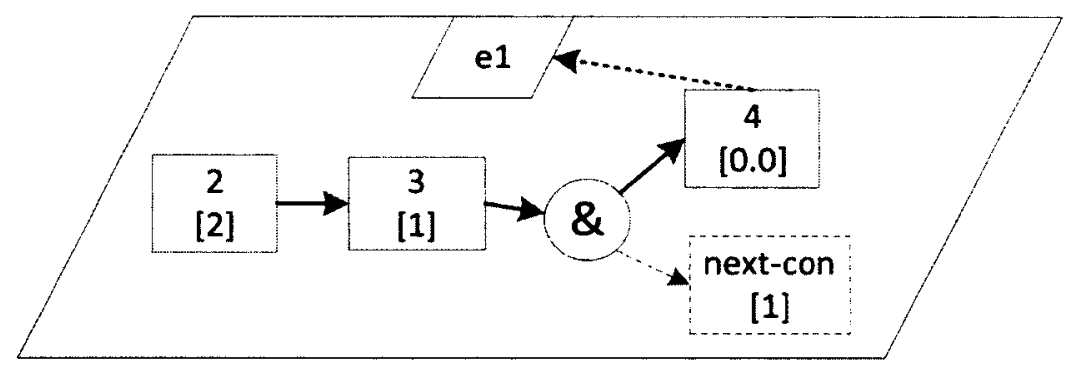

Figure 7-11 the PAG of an eg_connector with FA type end event

\subsubsection{PAG for an eg_connector with end event of type EN}

The top side of Figure 7-12 shows an example of an eg_connector with end event of type EN and the bottom side shows the PAG of this eg_connector. The first activity is driven by the preceding PAG and the end event of this eg_connector doesn't interface with any PAG.

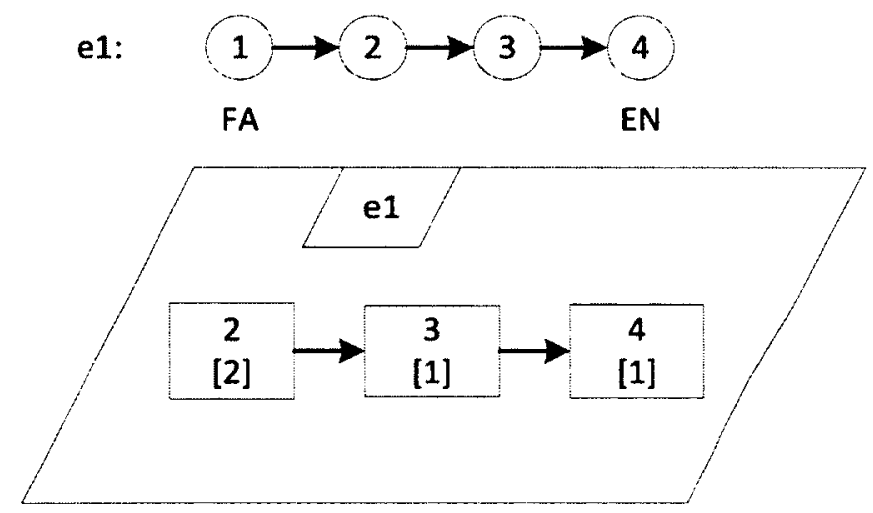

Figure 7-12 the PAG of an eg_connector with EN type end event 


\subsubsection{PAG for an eg_connector with end event of type FS}

The top side of Figure 7-13 shows an example of an eg_connector with the end event of type FS, and the bottom side shows the PAG of this eg_connector. The activity before the end activity uses the and-fork connection to fork into the end activity of this PAG and the precedence connection of the following first activity of the PAG. The specification of this activity is not included in this PAG but a precedence connection to that is included (this is why this activity is shown by a different line pattern box). The end activity sends a request to a server task.

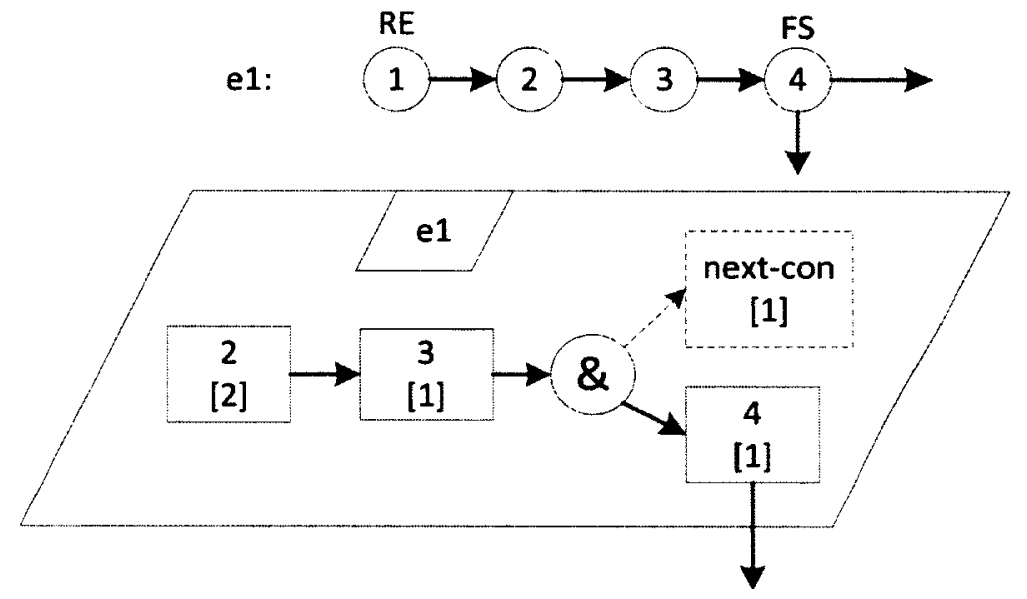

Figure 7-13 the PAG of an eg_connector with FS type end event

\subsubsection{PAG for an eg_connector with end event of type SA}

The top side of Figure 7-14 shows an example of an eg_connector with the end event of type SA and the bottom side shows the PAG of this eg_connector. The end activity of in this PAG sends a response to the entry of its PAG. This PAG doesn't have an interface with its following PAG. 


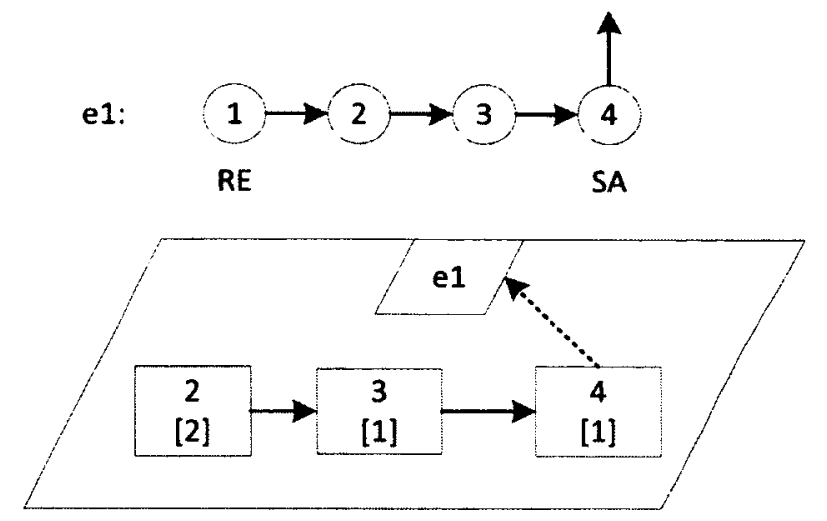

Figure 7-14 the PAG of an eg_connector with SA type end

\subsubsection{PAG for an eg_connector with head event of type FS}

The top side of Figure 7-15 shows an example of an eg_connector with the head event of type FS and the bottom side shows the PAG of this eg_connector. This eg_connector represents the operation after a non-blocking interaction. It will be followed by a bridging eg_connector (the bridging eg_connector is not part of this eg_connector). This PAG, despite other PAGs, has an interface with its preceding PAG. The interface is constructed using its following bridging eg_connector. The interfacing connection is an and-join connection in which one input is the end activity of this PAG and the other input is the activity of head event of this eg_connector. The specification of this activity belongs to the preceding PAG but the precedence connection from it belongs to this PAG (this is why this activity is shown by a different line pattern box). 


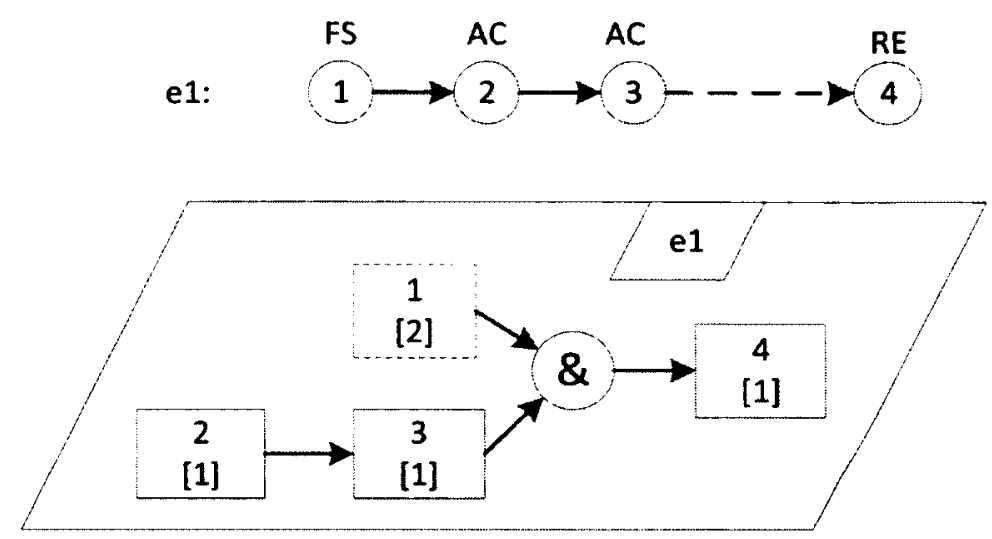

Figure 7-15 the PAG of an eg_connector with FS type head

\subsection{PAG construction algorithm}

Figure 7-16 shows the algorithm of construction the PAGs for the eg_connectors which have end events of SE, SA and FS. This algorithm can easily continue in the same way for the other types of eg_connectors. In order that an eg_connector can be processed with this algorithm, it needs to have at least 3 events. Therefore, eg_connectors will be padded with pseudo events, with zero service time, to make up for the minimum required event count. The following sub-sections explain how the parameters of the connections of the activities of SA, FA, SE and FS event types are determined. 
EVENT = the next event of the head event in the connector

Create an activity ICON for EVENT and transfer the CPU-time of EVENT into ICON

EVENT = next event in the connector

WHILE O

Create an activity ICON for EVENT and transfer the CPU-time of EVENT into ICON

Link previous activity to ICON

IF (EVENT is the last element in the connector)

EVENT = the event before EVENT

break

END IF

EVENT = next event in the connector

ENDWHILE

IF (connector_end_event_type $==$ "SE")

Determine the request rate

$D E S T$ ENTRY $=$ Specify the destination entry

Link connector_end_event to DEST_ENTRY

ELSE IF (connector_end_event_type $==$ "SA")

$S E L F \_E N T R Y=$ Specify the current entry of connector_end_event

Lind connector_end_event to SELF_ENTRY

ELSE IF (connector_end_event_type $==$ "FS")

Link EVENT to the and-fork connection

Determine the request rate

$D E S T$ ENTRY $=$ Specify the destination entry

Link one output connection of the and-fork to DEST_ENTRY

Use the other output connection for interfacing

ENDIF

Figure 7-16 algorithm of constructing the PAG of a connector

\subsubsection{Specifying activity replies}

An entry which accepts synchronous type message must generate a reply during processing of that message. Activities of types "SA" or "FA" must specify a reply. A 
reply includes the ID of an entry which is the entry in which that event is executed. This entry ID is identified from the attributes of the profile of that event.

\subsubsection{Specifying activity requests}

Activities of type SE or FS must make rendezvous or send-no-reply requests, respectively, to other tasks. This request has three parts;

1. The request type

2. The destination task/entry

3. The request rate of that activity

The first and second of the above attributes can be identified from the association of an event to the EI it is part of. To find this association, the event object has an attribute named the "EI_id". This attribute, for each event of an EI object, is set to the ID of that EI.

The request rate is determined from the TI to which the EI of an event is mapped to. This subject will be explained in sub-section 7.7.3.

\subsection{Activity graph construction}

The algorithm takes one eg_connector at a time from the ordered list of eg_connectors in the task-eg_connector-vector, creates its PAG, and appends it to that of the previous PAG until it reaches the end of the list.

This section briefly explains the details of request rates determination as well as the algorithm used to form activity graphs of entries. 


\subsubsection{Specifying the start activity of an entry}

The first event is identified from the first event of the first eg_connector in the taskeg_connector-vector of a task. The entry object has an attribute named the "first-event" which will be set to this value when it is identified.

\subsubsection{Algorithm}

The algorithm of Figure 7-17 will be applied to the task-eg_connector-vector of each task to construct that task's activity graph. For each eg_connector of this vector, first the eg_connector is padded, then the types of the end event and the head events of that eg_connector are identified. Finally this information will be used by the algorithm of Figure 7-16 to construct its PAG. This algorithm appends the activity specification and precedence relation of each new eg_connector to those of the previous ones.

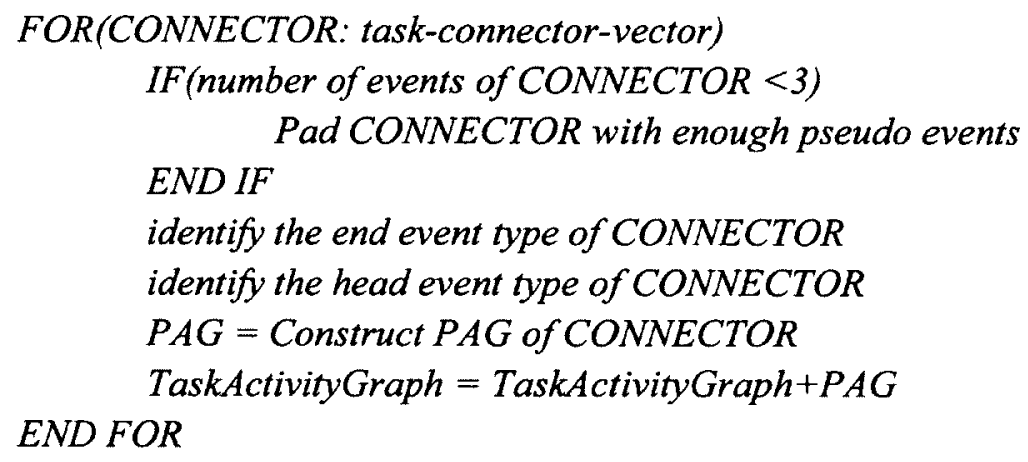

Figure 7-17 algorithm of construction the activity graph of a task 


\subsubsection{Request rate determination}

The average request rate of an activity from Task $\mathrm{A}$ is determined by two key parameters:

1. The total requests made from Task A to a different task, and

2. the total incoming requests to that entry.

The first parameter is represented by the total number of EIs whose client is Task A. Therefore, if only one activity interaction is used to represent all the interactions of an EI, the average request rate of Task A would be the average EI counts of all the EIs whose client task is Task A. In an activity graph, a representative EI is used to represent all the interactions, and the following equation is used to determine the request rate of a task:

request rate of Task $A=$

$\frac{\text { average EI counts of all EIs sourced from Task } A}{\text { EI count of the representative EI of Task } A}$

Equation 7-1

The TIs of a computation are used to classify the interactions between the two tasks and determine demands associated with them. The TI has a vector, called the "EI vector" to store all EIs which map to it. The contents of this vector are used to determine the request rate of activities. The TI class has the average of request rates. The average of the single-EI counts of the EIs in the EI vector determines the request rate. An association is also made between each EI and the TI it maps to, which is used to make the average request rate attribute accessible by an EI. 


\subsection{Example: graph transformer}

In this example, the activity graph of a two tier interaction between tree tasks whose execution graph is shown in Figure 7-18 is constructed. Task 1 is the reference task, playing the role of a pure client which creates the initial request to the system. Task 2 is a non-blocking server which demands for the services of Task 3 through asynchronous requests. Task 2 also performs a second phase operation after servicing requests of Task1. Task 2's second phase operations involve nested interactions with Task3. Task3 is a pure server task which services requests of Task2. This task also performs a second phase of operations. It is assumed that the events involved in interactions, such as $2,6,8$, $19, .$. are solely used for this purpose and their consumption of CPU time is represented by activity events which are then merged into other activity events. All three tasks execute on their own processors.

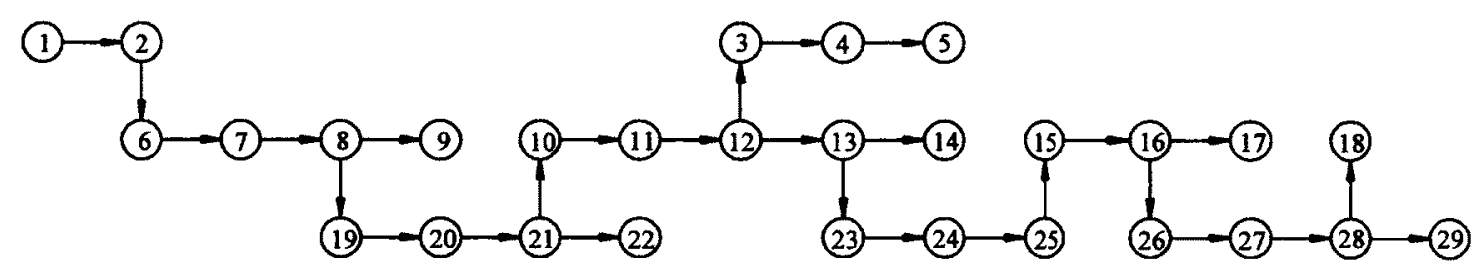

Figure 7-18 execution graph of the test case

\subsubsection{Implementation}

The internal activities of the tasks are emulated by mathematical operations. The Java Management Factory class [66] was used to measure the pure time consumed by activities. An event-trace is logged after an event is executed. This operation adds one 
line to the event-trace file of each task. An instrumentation object is created, using Java, which contains all the necessary methods for the instrumenting this simulation. An instance of this object was used for instrumentation of each task.

The timing to make a service request to the reference task is simulated using an exponential density function which mimics the inter-arrival time between requests received by that task. The pseudorandom number generation (RNG) of Uncommon Math libraries [67] is used to implement this density function. Uncommon Math uses an algorithm called Mersenne Twister (MT) [68] which is used for generating uniform pseudorandom numbers. This algorithm provides a period as large as $\left(2^{19937}-1\right)$ and $623-$ dimensional equivalent distribution up to 32-bit accuracy, while using a working area of only 624 words.

The tasks, which are implemented separately, interact with each other using Java socket technology [69]. A server task blocks at a "server socket" until it receives a message which contains the most updated timestamp of the sending task. The messages between the tasks are Java objects which are serialized using Java object serialization technology [70] and transferred between the sockets of the tasks.

The tasks are concurrent. The Java high level API interfaces of the "Java concurrent package" such as BlockingQueue [71], were used to facilitate synchronization of resources utilization between the threads. Each server task consists of a "socket server" thread and a pool of worker threads as well as one logger thread. The main program creates an instance of thread-safe BlockingQueue and passes its reference to the server, workers and logger threads. The server thread takes care of the socket server which is 
blocked waiting for the arrival of a request from a client. Once the request is received, the server thread spawns a worker thread which takes the clients socket reference to interact with it. This worker thread receives the service request, performs some internal processing and, if necessary, starts the interaction with a different server which takes over part of the required service. When the reply is received, the worker thread wraps up the interaction by sending over the results to the client before going to a second phase of operation.

The workers write lines of strings of event-traces to the BlockingQueue, using its put() method. This process is inherently synchronized by the BlockingQueue interface. The run () method of the logger thread waits until all the events are logged, then is notified by the queue, reads the events from the queue by calling the queue's take() method, and writes them into the event-trace file. The logger thread is shut down by a special string that it reads from the queue, which was intentionally added by the last worker thread. In this way, a busy thread is able to add an event-trace to the queue of event-traces and at moments when the server is less busy, the logger thread will pick the event-traces from the queue and log them to disk.

A deterministic scheme was implemented for verification of the system, in which Task 1 sent only one request, Task 2 performed one nested interaction with Task 3 and then replied to that request from Task 1 . Task 2 then performed two other nested interactions with Task 3 to do the remaining processing in a second phase. 


\subsubsection{Results}

Figure 7-19 shows the activity graph automatically produced for this system. The information such as the speed of CPUs, their multiplicity, scheduling policy and mappings to resources was externally provided. Activities with zero CPU demand are those which were involved in interactions as well as some padding activities. As can be seen, all request rates are 1.

\subsection{Summary}

Activity graphs allow for accounting the effect of utilizing parallelism techniques in LQN performance models. This chapter introduced an approach to automatically construct activity graphs of distributed applications. This approach used the techniques introduced in Chapters 4, 5 and 6 to transform the simple entry structure of tasks in an LQN model into an activity graph. This approach transfers the CPU consumption of events into their corresponding activities and automatically determines the request rate of each activity. The final system is able to create the activity graph of a distributed application in an automated fashion from its events log. 


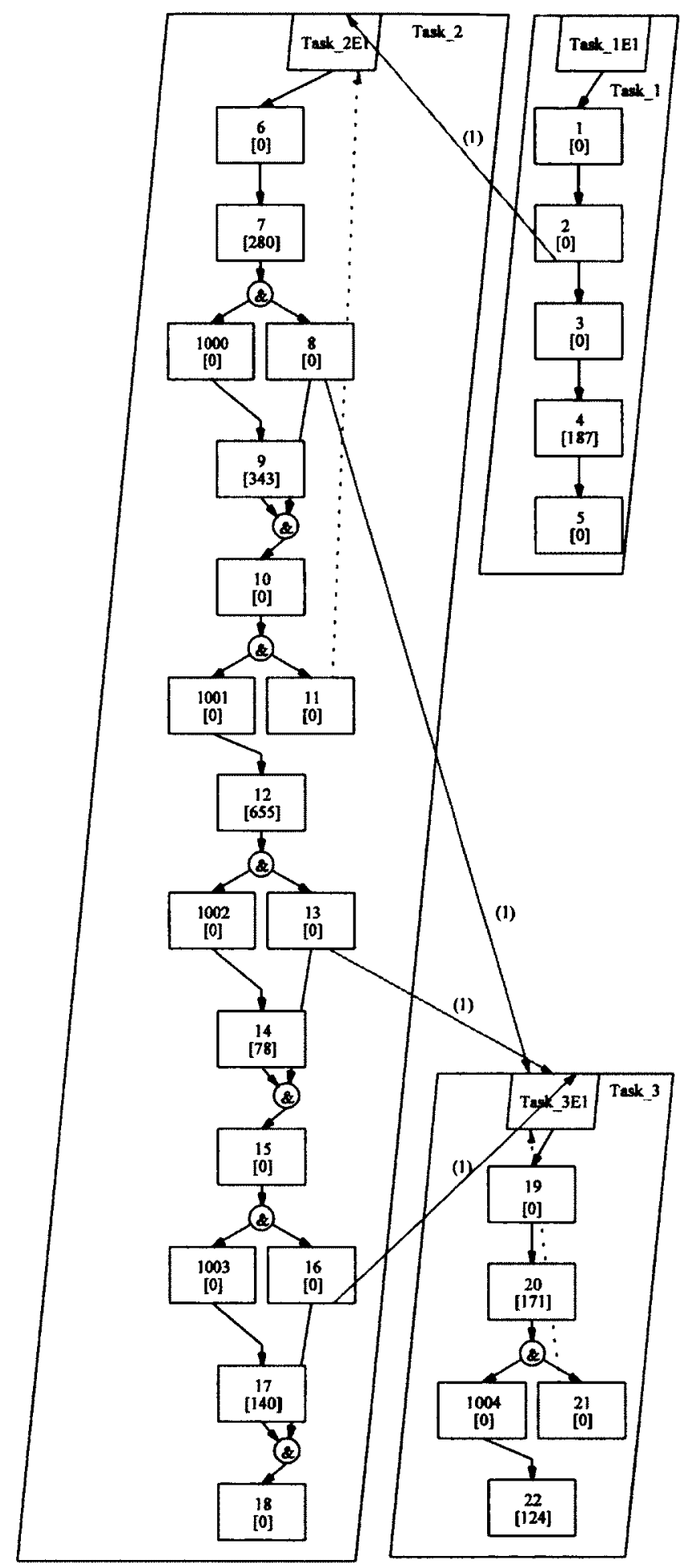

Figure 7-19 activity graph of the graph transformer example 


\section{Chapter 8: Parasol Case Study}

This case study is to validate the performance model created from the application of WebTime on the simulated version of a distributed system. The performance model of the distributed system, called the "base model", is known in advance because the distributed system is simulated using the base model.

The system under test in this study is called Building Security System (BSS). BSS is intended to control accesses to the doors and to monitor human activities in a building like a hotel or a laboratory. BSS consists of a number of cameras located around a building, a number of door locks, card readers and access cards, an application server and a database server. Figure 8-1 shows the deployment diagram of BSS.

Performance analysis of BSS was previously conducted ([72][73]), but the approach used in this chapter is different. In [72] the authors created an LQN model of BSS from the description of BSS and then modified the model in several steps until the desirable performance was achieved. In [73], the authors simulated BSS from its LQN model and then directly used the simulated system to study the performance behaviour of BSS. In this chapter BSS is first simulated, using the same tool which was employed by [72], and then the simulated system is instrumented to get it to generate event-traces while it was running. The event-traces were then used by WebTime to construct the LQN model of 
the simulated BSS. To demonstrate the capability of WebTime, the resulting LQN model was compared with the model which was adopted from [73].

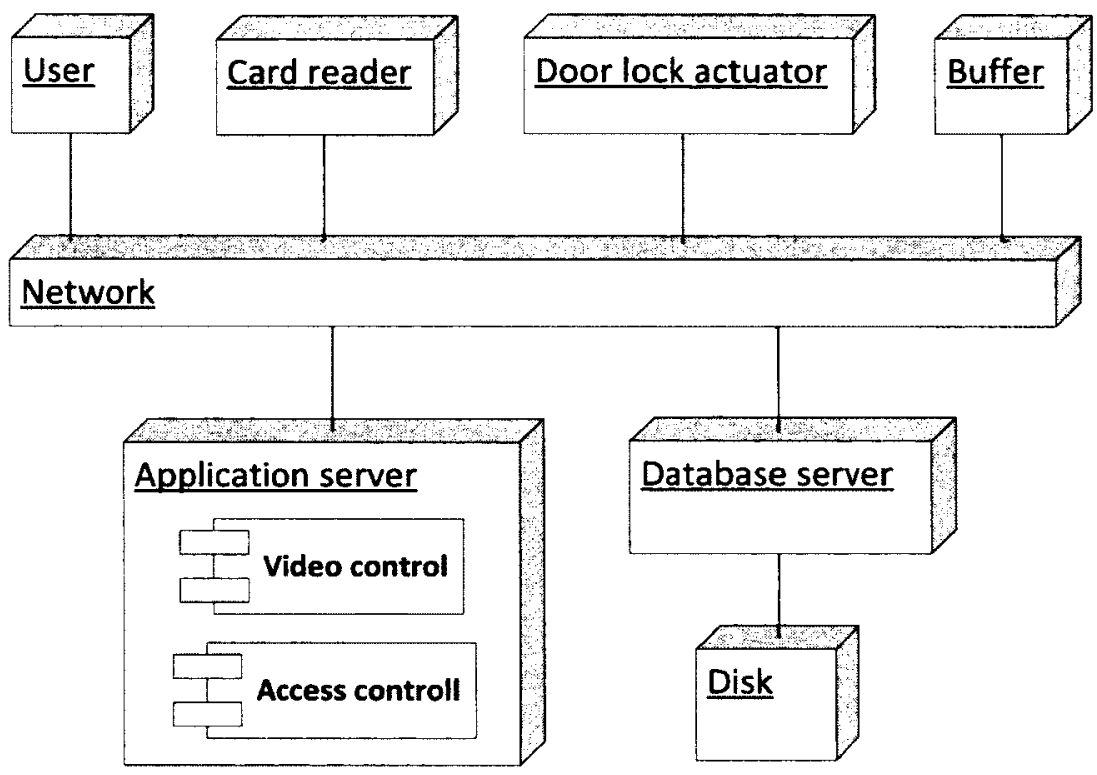

Figure 8-1 deployment diagram of a BSS

\subsection{BSS}

Two main operational behaviours, or scenarios, of BSS, called "door access control" and "video control", are modeled here. Figure 8-2 shows the sequence diagram of interactions between the tasks in scenario "door access control". In this scenario a number of door locks are controlled by access cards. The tasks involved in this scenario include: "user", "card reader", "access control", "door lock", and "database". All the tasks, except "user", are concurrent with concurrency levels of $50,60,10$ and 2 for "card reader", “access control", “door lock”, “database”, and “disk” respectively. Task "user” inserts a card into the door-side "card reader" where its content is read and transmitted to 
"access control" in the application server. "Access control" checks the access rights associated with the card by sending a query to "database" in the database server. If an access right is available, the lock actuator associated with the card reader is triggered, otherwise an access is denied. This scenario has a 5-tier architecture.

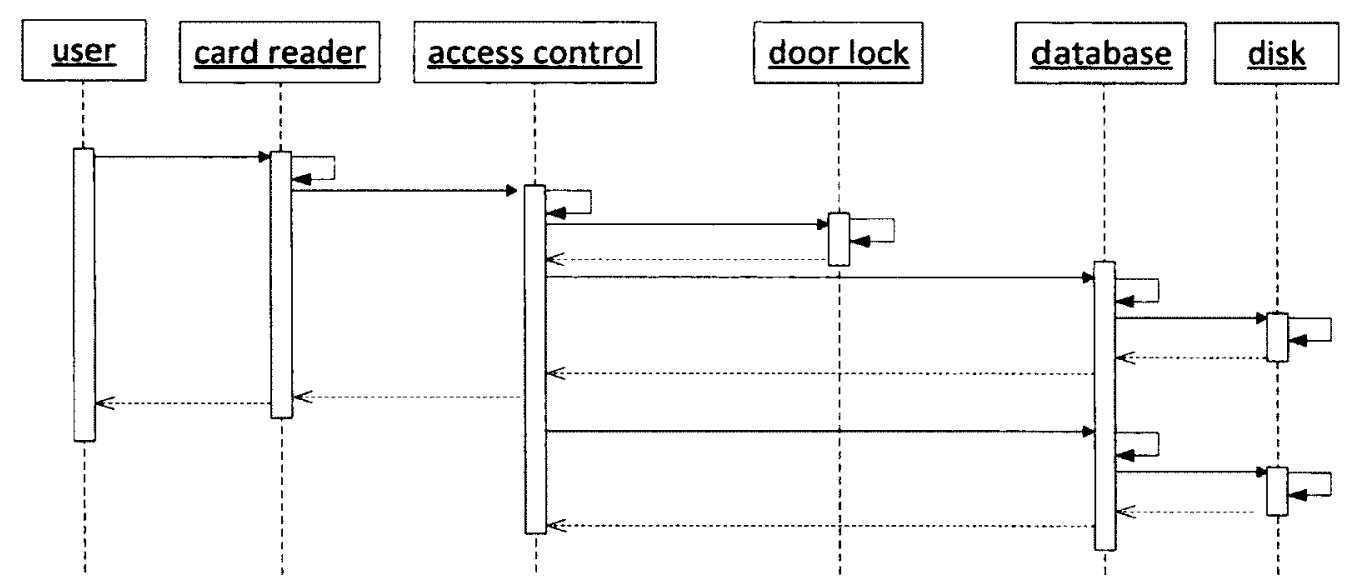

Figure 8-2 Sequence diagram of the door access control scenario

The request rates of the interactions of the access control scenario are shown in Table 8-1.

Table 8-1 request rates of the tasks involved in "door access control"

\begin{tabular}{|l|l|c|c|}
\hline Requesting task & Requested tasks & Request rate/phase1 & Request rate/phase 2 \\
\hline user & Card reader & 1 & 0 \\
\hline Card reader & Access control & 1 & 0 \\
\hline Access control & Door lock & 1 & 0 \\
\hline Access control & Database/entry1 & 1 & 0 \\
\hline Access control & Database/entry2 & 0 & 2 \\
\hline Database/entry1 & Disk/entry1 & 0.4 & 0 \\
\hline Database/entry2 & Disk/entry2 & 1 & 0 \\
\hline
\end{tabular}


In the second scenario, "video control", video frames are periodically captured from the cameras and transferred to the database server. Figure 8-3 shows the sequence diagram of this scenario. The tasks involved in this scenario include: "video control", "acquire process", “buffer get”, “buffer”, "get image", "network", "pass image”, "store process", "buffer release", "database", and "disk". "Video control" generates a workload by sending service requests to "acquire process". "Acquire process" checks for availability of buffer through "buffer manager", requests an image through "get Image" and "network", and then wraps up its operation by passing the control to "store process" through "pass image". "Store process" then takes over, puts the image in "database" and releases "buffer" through "buffer release". "Buffer", "store process", "acquire process", "database" and "disk" are all concurrent with concurrency levels of 15, 3, 12, 10 and 2 respectively. The types of interactions in this scenario are Synchronous and Forwarding. Most of the tasks perform part of their processing in a second phase to provide quicker response to their clients. 


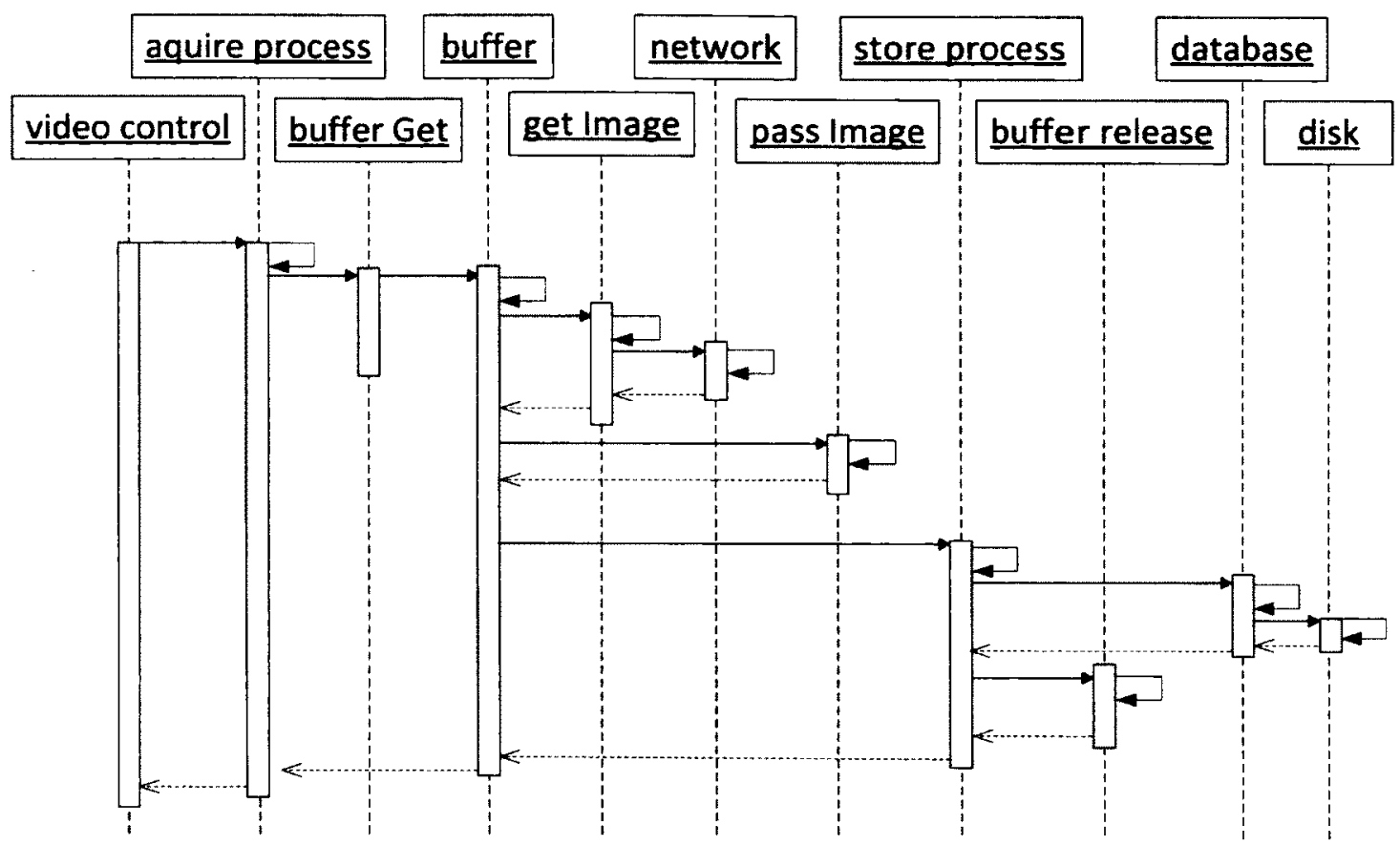

Figure 8-3 Sequence diagram of video control scenario

Tasks "database" and "disk" are shared by both of the scenarios. The request rate of each interaction in "video control" is shown in Table 8-2.

Table 8-2 request rates of the tasks involved in Scenario "video control"

\begin{tabular}{|l|l|c|c|}
\hline Requesting task & Requested task & Request rate/phase 1 & Request rate/phase 2 \\
\hline Video control & Acquire process & 10 & 0 \\
\hline Acquire process & bufGet & 1 & 0 \\
\hline bufGet & buffer & 1 & 0 \\
\hline buffer & Store process & 0 & 1 \\
\hline buffer & Pass image & 1 & 0 \\
\hline buffer & Get image & 1 & 0 \\
\hline Get image & network & 1 & 0 \\
\hline Store process & Buffer release & 1 & 0 \\
\hline Store process & Database / entry 3 & 8 & 0 \\
\hline database & Disk / entry 3 & 8 & 0 \\
\hline
\end{tabular}




\subsection{Simulation}

The tool which was used to simulate BSS is Parasol [74]. Parasol is a discrete event simulator tool for simulating distributed or parallel applications. The execution environment of Parasol is built up of multi-processor nodes, interconnected with communication devices of various types and capacities. The tasks in this tool must be written in the "C" language. These tasks may represent both application and operating system software. A simulation driver looks after running the simulation. Parasol provides random variable generation and statistical collection tools to capture performance behaviours in a fashion equivalent to making measurements. The kernel in Parasol provides features for defining heterogeneous network topologies of arbitrary complexity; dynamic task structures with a variety of management tools including task migration; inter-process communication using communication ports; and spinlocks for low-level task synchronization [74].

BSS was simulated by writing and adding a C program, called here the "main program", to Parasol. The main program:

- Defined the nodes of BSS which include application server, database server, disk, acquire process, network, card reader, door lock actuator and buffer

- Defined the software tasks involved in BSS: "video controller", "acquire process", "bufGet", "buffer", "get image", "network", "pass image”, “store process", "buffer release", "card reader", "access control", "door lock", "database", and "disk"

- activated all the tasks 
A task in the main program is mainly characterized as a two-port entity which can send messages to and receive messages from other tasks. One port of a task is a standard port for listening to the requests made to this task and the other one is an acknowledge port for listening to the response of a different task to which this task sent a request.

Operational behaviours of a task in Parasol depend on the type of that task. Two types of tasks were defined: reference and server tasks. A reference task has two operational states: sending a request to a different task and then listening to its acknowledge port. When activated, a reference task goes into its first state, sending a message, and then goes and remains in its second state until receiving a response message. This message brings this task back to the first state, as shown in Figure 8-4.

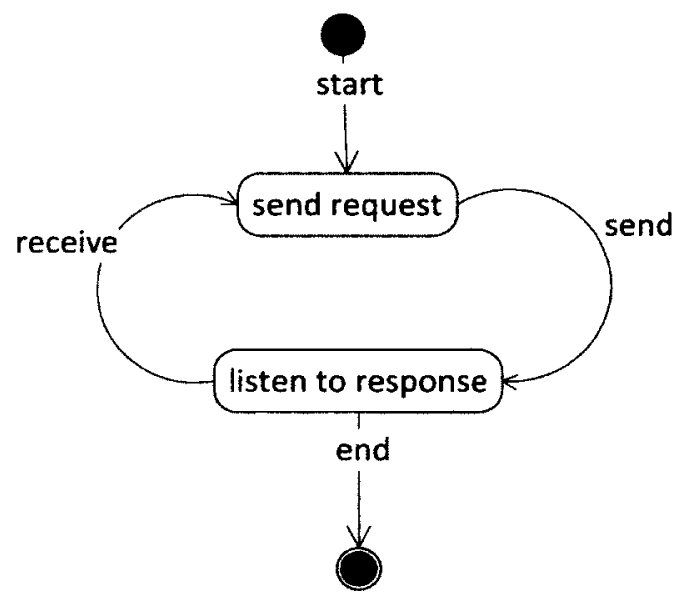

Figure 8-4 state diagram of a reference task

A server task starts by listening to its standard port when activated. Two types of server tasks are used in this work: pure and active. A pure server task simple services the 
request it receives. It replies to the task from which it receives a request and then goes back to its initial state, listening to its standard port, as shown in Figure 8-5.

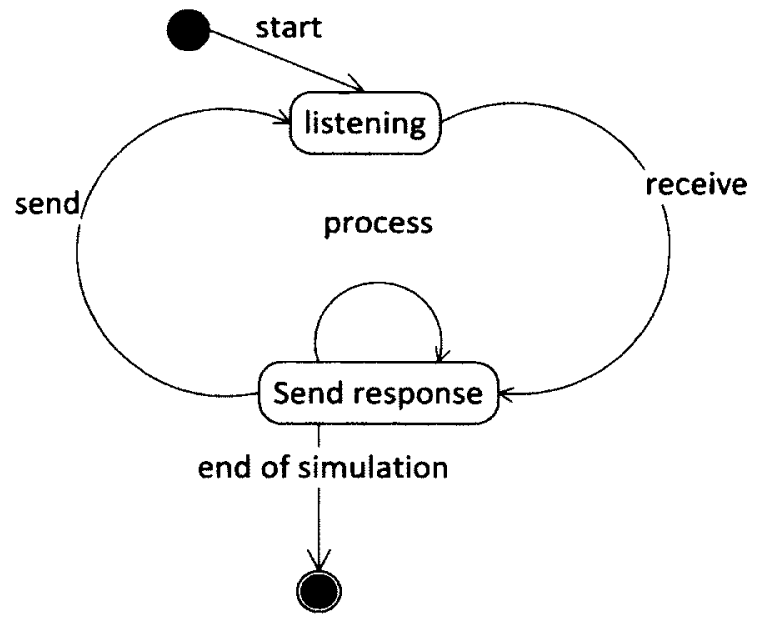

Figure 8-5 state diagram of a pure server task

An active server task makes interactions with other server tasks in order to service the request it receives. This task sends a request to a third server task after receiving the request and remains listening to its acknowledge port. Once this task receives a response, it replies to the client task from which it received a request in the first place and turns back to its initial state, as shown in Figure 8-6. 


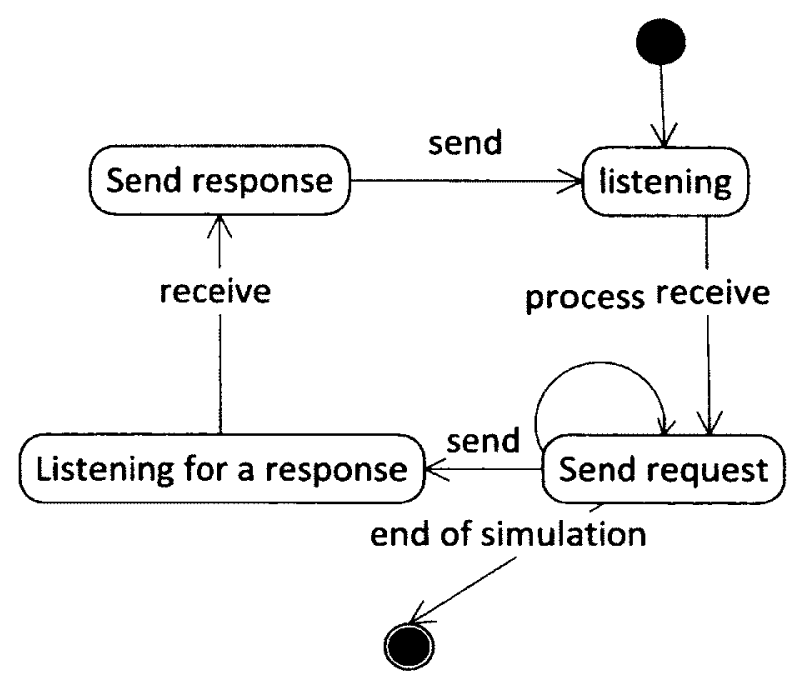

Figure 8-6 state diagram of an active server task

Parasol was extended to simulate a multi-threaded task, because originally it didn't have a ready construct for that. A multi-threaded task is constructed using a single task, here called the "worker-task", for each thread. These worker tasks are then managed by a different task, called here the "manager-task", which represents the multi-threaded task. The manager-task has a task-pool which contains a reference to each of the worker tasks. Once a request is received by the manager-task, it passes the reference to that request to one of the free worker-tasks in its task-pool. The worker-task then picks up the request and starts processing it. When the worker-task has processed the request it receives, it sends a reply back to the client task directly and signals the manager-task to put the worker-task's reference back into the task-pool. The discipline by which a free task in selected from the task-pool is LIFO in this work. 
The tasks of BSS are configured to operate as the reference, the pure server and the active server. Each task is associated with a number of entries. Each entry is also associated with two operational phases. For each phase of an entry, the following parameters are defined:

- The number of destination tasks

- The destination tasks for each entry

- The entry of each destination task

- The visit ratio to each destination task

- The CPU consumption of each destination task

The workload is generated by "user" and "video control", independently, by sending service requests to their corresponding lower layer tasks, "card reader" and "acquire process" respectively. The request rates are specified by an exponential random delay time between each request made by these tasks.

There can be more than one interaction in a row between a client and a server task. This interaction pattern is called the "cluster interaction". The number of interactions in a cluster interaction is determined by an exponentially distributed random variable whose mean value is the expected number of interactions between the two tasks, which is specified by the call rate parameter.

\subsection{Instrumentation}

Parasol is instrumented to monitor the key events from which the main architecture of BSS can be reconstructed. These events are: 
- Send message.

- Send acknowledgment message.

- Receive message.

- CPU-time consumption activities.

The instrumentation of Parasol and monitoring of its events was straightforward. Originally Parasol has a function to $\log$ a simple form of an event-trace. This function was modified to $\log$ an event of WebTime. This function was then used as a software sensor in all the places where an instrumentation was required.

Parasol had two functions for sending and receiving an event: ps_receive_t () and ps_send $t()$. These functions and their signatures were modified to accept and carry the attributes of a WebTime' timestamp object. These attributes were defined by a C struct named "OTS". Finally the structure of a message in Parasol was modified in order to carry an instance of OTS.

A number of functions were created to add suitable monitoring software sensors in various locations in Parasol. Table 8-3 contains the name of these functions and the event types they monitor. The locations where these functions are inserted are shown in Figure 8-7. This figure uses the abbreviated names of the events which are shown in Table 8-3. Effectively the monitoring functions sit exactly where ps_receive_t() or ps_send_t() exist in the program. Therefore, the ps_receive_t() or ps_send_t() functions were included in each of the monitoring functions, where appropriate, as shown in Table 8-3. The last thing to do was replacing ps_compute_t (),ps_send_t () and 
ps_receive_t () in the main program with a suitable monitoring function according to Table 8-3

Table 8-3 monitoring functions and the events they monitor

\begin{tabular}{|l|l|c|l|}
\hline $\begin{array}{c}\text { Monitoring } \\
\text { function }\end{array}$ & Event to monitor & $\begin{array}{c}\text { Abbreviation } \\
\text { for the event }\end{array}$ & $\begin{array}{c}\text { Includes parasol } \\
\text { function of }\end{array}$ \\
\hline Process() & activity & AC & ps_compute_t() \\
\hline Send() & send & SE & ps_send_t () \\
\hline sendAck() & Send acknowledgment & SA & ps_send_t () \\
\hline forkAck() & Fork acknowledgment & FA & ps_send_t () \\
\hline Receive() & receive & RE & ps_receive_t () \\
\hline External() & external & EX & none \\
\hline
\end{tabular}

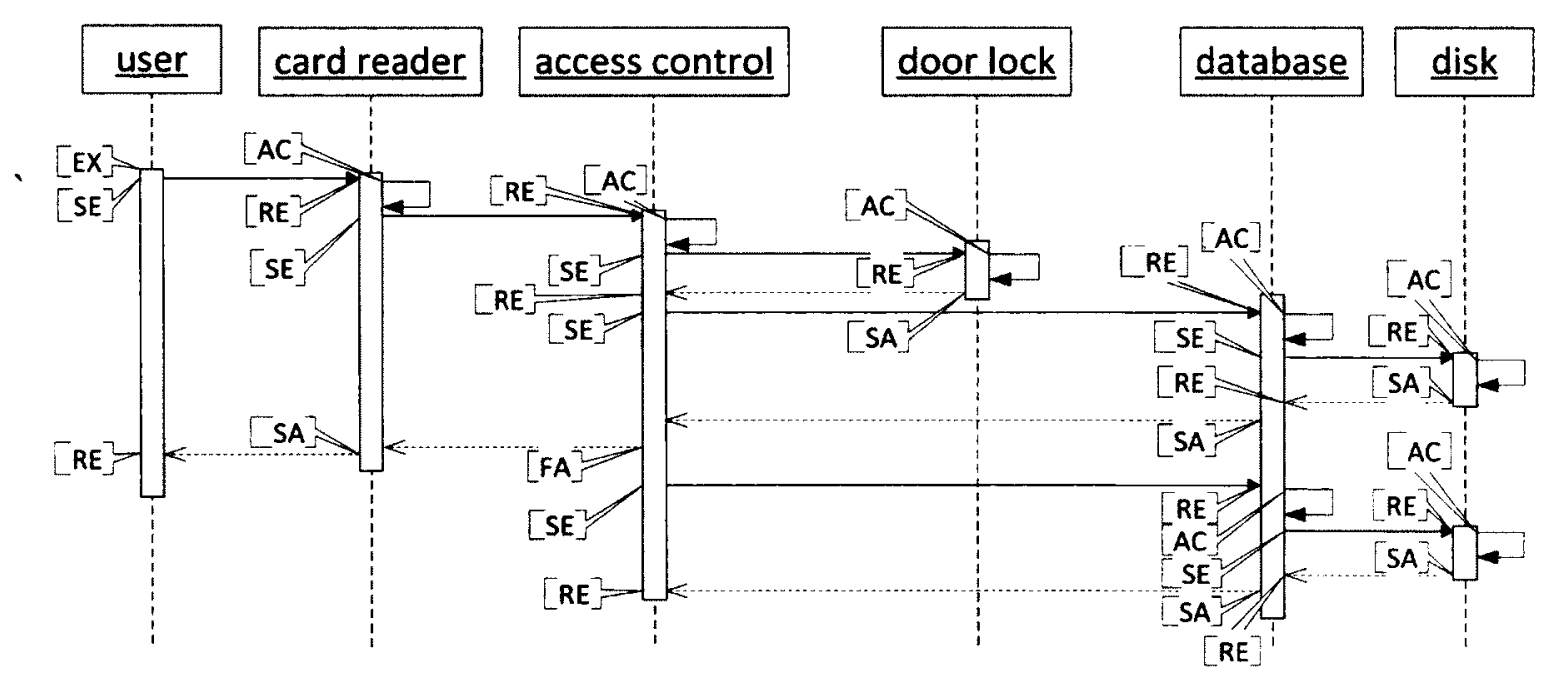

Figure 8-7 monitoring sensor locations of the tasks involved in "access control" scenario 


\subsection{Experiment}

The events $\log$ of the created simulation program can be used to reconstruct the performance model of BSS. This model can then be solved to analyze the performance behaviours of the BSS. This analysis can include the determination of the response times of various tasks, identification of performance bottlenecks and suggestions for reconfiguring and rearchitecting the original BSS for better performance. Since this analysis was already performed on the base model by the authors of [73], it was decided to validate WebTime through comparing the reconstructed model with the base model in this case study.

To reconstruct the performance model of BSS, the simulated program was run for 20,000 simulation seconds to collect WebTime event-traces. Figure 8-8 shows a small sample of these event-traces.

After running the simulation, the resulting events log was fed to the WebTime analyzer; it identified tasks, entries, the interactions between the entries and the count of interactions performed in the simulated application, and it constructed the performance model of the system. The LQN performance model of this case study is shown in Figure 8-9. This model is very similar to the base model which was adopted from [73].

\subsection{Results}

WebTime was used to create the performance model of the simulated version of BSS. The resulting model was exactly the same as the base model from which the simulation program was created. This demonstrates the capability of WebTime to automatically reconstruct a performance model from the event-traces extracted from that model. 


$\begin{array}{llllllllll}\begin{array}{l}\text { Invo- } \\ \text { cation } \\ \text { index }\end{array} & \begin{array}{l}\text { Parent } \\ \text { thread }\end{array} & \begin{array}{l}\text { Thread } \\ \text { index }\end{array} & \begin{array}{l}\text { Event } \\ \text { index }\end{array} & \begin{array}{l}\text { event } \\ \text { type }\end{array} & \begin{array}{l}\text { task } \\ \text { name }\end{array} & \text { Entry } & \begin{array}{l}\text { CPU } \\ \text { time }\end{array} & \begin{array}{l}\text { Multi- } \\ \text { plicity }\end{array} & \text { CPU } \\ \text { name }\end{array}$

Figure 8-8 a few event-traces of the simulated BSS 


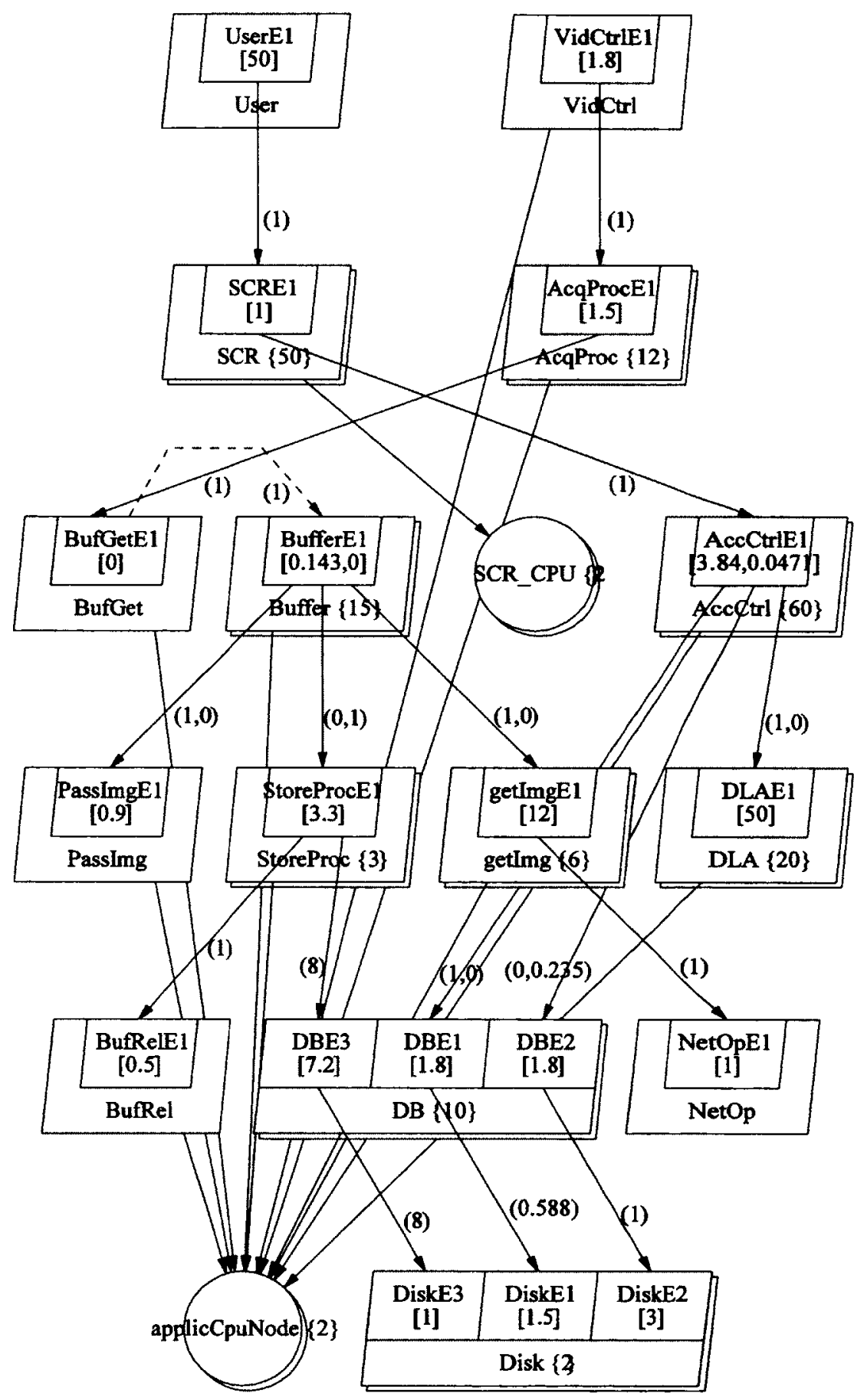

Figure 8-9 the reconstructed LQN model of BSS, created from the base model adopted from [73] 


\section{Chapter 9: Java BSS Case Study}

This chapter is to analyze the performance behaviours of the prototype of a distributed system using WebTime. The system under test is Building Security System (BSS) which was described in Section 8.1 but implemented using Java socket technology here. BSS contains various interaction types against which the interaction identification capability of WebTime can be tested. The socket communication is flexible enough to implement these interactions.

\subsection{Implementation of BSS}

Scenario "Video control" of BSS was implemented and deployed in two different computers. These computers were connected through a LAN network, as shown in Figure 9-1. The tasks in this implementation are pseudo tasks, in the sense that they represent real tasks by performing the following three basic functions:

- listening to a socket port to receive a message as soon as it arrives,

- processing the message by consuming a certain amount of CPU-time, and

- responding to the task from which the recipient task received a request message.

As can be seen in Figure 9-1, this system has two nodes: application server and database server. The application server is an Intel Core2 Duo running at $2.10 \mathrm{GHz}$, and using Linux Ubuntu as its operating system. The BBS tasks "Video control", "Acquire 
process", "Buffer", "Store process", "Buffer release", "Get image", and "Pass image" were deployed here. Task "buffer" was also included on this node although in the real application it was separate. "Buffer" doesn't consume CPU-time. Therefore, it shouldn't be a possible host CPU bottleneck. The database server is an Intel core i7 computer running at $2.6 \mathrm{GHz}$ with Linux Ubuntu as operating system. Tasks "database" and "network" were deployed here. The database server and application server nodes were connected through a LAN.

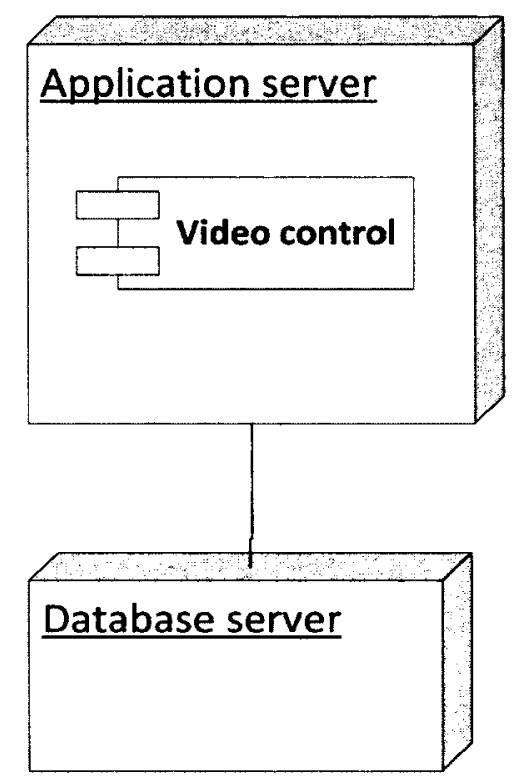

Figure 9-1 deployment diagram of "video control" scenario

\subsection{Monitoring system}

A monitoring system was designed to generate and collect event-traces of BSS which were used by WebTime. The monitoring system consists of two sub-systems: event-trace generators and event-trace loggers. The event-trace generators are the tasks of BSS which 
were instrumented to generate the event-traces. There is one event-trace logger per task to collect event-traces of that task. The logging system consists of a logger thread and a queue, called here the "trace-queue", shown in Figure 9-2. The "trace-queue" was implemented using objects from the "Java concurrent package" . A reference to a tracequeue is passed to its corresponding task so the task knows where to send its event-traces. The trace-queue is of blocking type to prevent simultaneous access by various threads of its task. When an event-trace is added into a trace-queue, the logger thread is notified of the arrival of the event-trace. As soon as there is less activity in the task, the logger thread takes these event-traces out of the trace-queue and puts them into the file system. The events logs of all the tasks are then used to construct the performance model of the whole system.

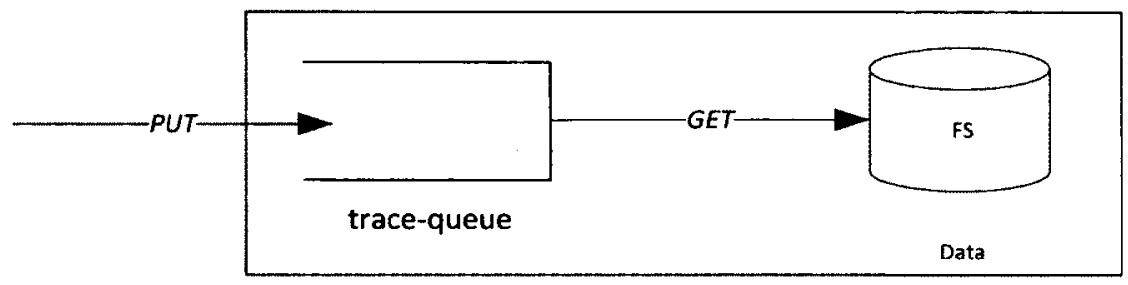

Figure 9-2 logger thread

The tasks of BSS are homogeneous multi-threaded active servers which are implemented using socket programming [69] and Java multi-threaded programming technologies [75]. These tasks were constructed using a manager and a number of worker threads. 
The main responsibility of a manager thread is to receive requests for services from other tasks and distribute them to its worker-threads. A manager thread consists of a socket port-server thread, a socket-queue, a worker-feeder thread and a worker-queue. The general template of a manager thread is shown in Figure 9-3. The port-server thread blocks at the input port to receive requests from clients. Once a request for service is received, the port-server thread adds the received socket object into the socket-queue. The worker-feeder thread is responsible for distributing the received socket objects among the worker-threads whose references are in the worker-queue. The worker-feeder constantly checks both the contents of the socket-queue and the worker-queue to find a client socket and a free worker-thread for servicing it. If the average demands for a task is more than the inverse of the total average service times of its worker threads, the socket queue is filled up and a bottleneck is created.

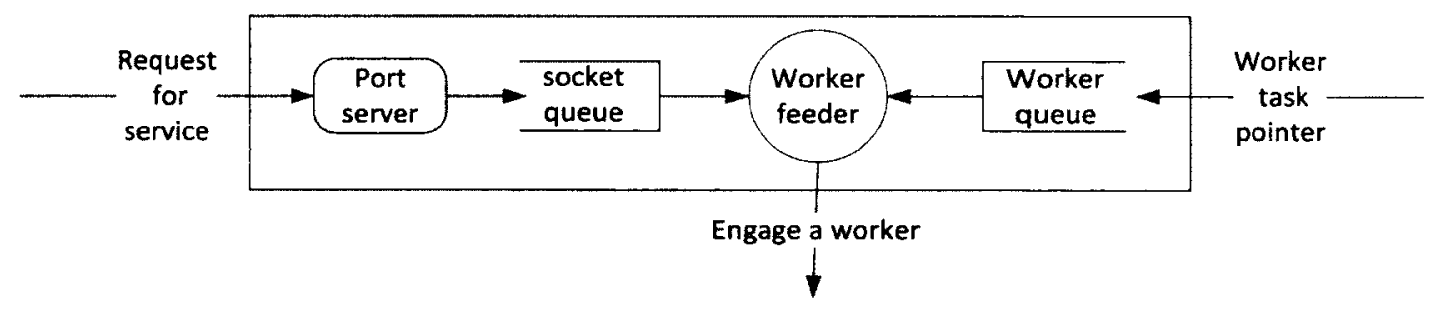

Figure 9-3 structure of a manager task implemented in Java

Each worker-thread is a complete active server, instead of a pure server, i.e. it receives a request from an initiating task, services the request and, if necessary, sends a 
request for service to a different task as part of its service. As soon as the servicing phase is finished, the worker-thread sends a reply back to the initiating task.

A worker-thread is instrumented to generate event-traces. When a manager thread of a task spawns a worker-thread, the manager passes the reference to the trace-queue of its task to the worker-thread so that the worker-thread can stream its event-traces to the trace-queue. Figure 9-4 shows the general architecture of a client worker.

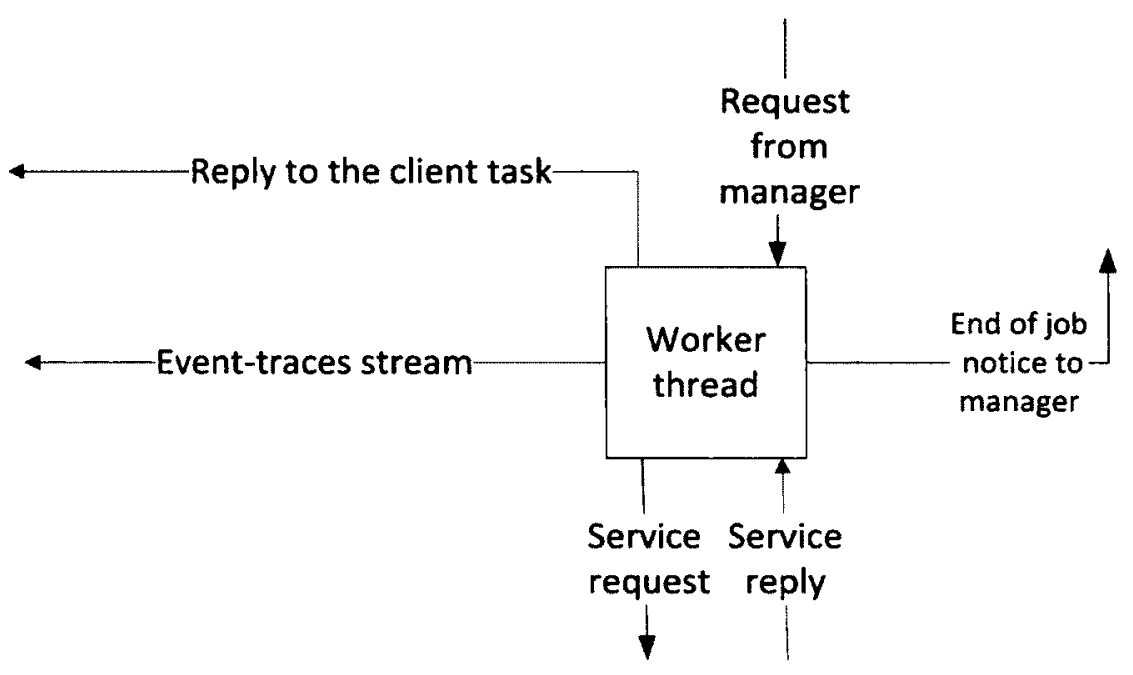

Figure 9-4 architecture of a worker thread

When a multi-threaded task is initialized, its manager and all of its worker-threads are initialized and the references to the worker-threads are added to the worker-queue of the manager task. This initialization helps to avoid the delay associated with on demand generation of the worker-threads in the middle of a process. 
As soon as a worker-thread is passed the received socket object of a client, this worker-thread is engaged with the requested services and its reference is removed from the worker-queue of its manager task. The worker-threads which finish serving their requests go into a waiting state again and add their references back to the worker-queue of their manager tasks.

\subsection{Software architecture}

All inter-task communications are point to point message passing. Each task has a message queue, which is actually the socket-queue of its manager task, in which messages are queued in a FIFO manner. A message is a Java object which carries the service request or the processing result of a task, as well as the most recent timestamp of a task. A message object is serialized using Java serialization technology [70] and transferred using Java software sockets [69]. A number of methods were created to accomplish various operations such as a message transfer activity at both receiving and sending ends using socket technology. These methods, called the "event methods", were used to implement various interaction types such as synchronous and asynchronous remote procedure calls, as well as CPU consumption activities. Table 9 -1 shows the list of event methods, the function they perform and the acronym for the event types that they represent. Event methods were incorporated into a Java class called "Interaction". 
Table 9-1 Event methods and their correspondence with events types

\begin{tabular}{|l|l|l|}
\hline $\begin{array}{l}\text { Interaction } \\
\text { method }\end{array}$ & method function & $\begin{array}{l}\text { Acronym for } \\
\text { event type }\end{array}$ \\
\hline send() & Synchronous service request & "SE" \\
\hline sendAck() & synchronous service result sends & "SA" \\
\hline fork() & asynchronous service request, & "FO" \\
\hline forkAck() & asynchronous service result send, & "FA" \\
\hline receive() & Receive a request & "RE" \\
\hline process() & Process activity & "AC" \\
\hline external() & External invocation trigger & "EX" \\
\hline
\end{tabular}

Most of the communications between the tasks in the application use Synchronous calls. Task "buffer" performs part of its operations in a second phase, after it has sent a reply to its client, "acquisition process". In this phase "buffer" calls "store process" as part of its processing.

"Video Ctrl" is the initiating task by which the whole process is started when an external request is received from a camera. "Video Ctrl" is multi-threaded to get the system to process overlapping requests, which happen when multiple cameras trigger the system for storing their video frames.

The processing events of tasks are simulated by mathematical operations to consume CPU-time. In order to make the CPU of a node busy for a certain time duration, this operation is looped as many times as needed. The loop iteration number is adjusted each task in order to obtain approximately the same model as [73]. For simplicity it is assumed that the send and receive events of a task are atomic, occur in real time without delay and 
consume no CPU-time. However, in the real world these events consume a small amount of CPU-time and their delays should not be ignored. This is one factor which should be considered to explain possible inaccuracy in the performance prediction by a model constructed using WebTime.

\subsection{Instrumentation}

A Java class named "Instrumentation" was created which contains all the instrumentation methods for various events. Instrumentation code was inserted where the event methods of BSS were executing. An instrumentation method is passed a timestamp object. When a timestamp is received, its contents are first logged, and this object is modified for the subsequent event. For the send and send-acknowledge events, this timestamp is carried by the message and recovered at the destination. For the fork and fork-acknowledge events the timestamp object is cloned so that it can be sent through the spawned thread at the forking point. Both the base and cloned timestamp objects were modified for their subsequent events.

Many parameters of an event profile are readily available or can be extracted from the operating system, including the CPU ID of the host, the CPU multiplicity, the task name and the count of client workers for multi-threaded tasks. An event type is set by the event method in which the event is executed. The CPU-time of an event is measured using the Java Management Factory class [66]. This class provides support for monitoring a thread contention and CPU-time. The CPU demands of tasks are only measured for activity events ("AC"). It is assumed that other events don't significantly consume CPU-time. 
The thread count is directly extracted from the thread multiplicity of a task (a task was set to create a certain number of client worker threads at its start up.).

\subsection{Experiments}

The system was run to generate an events log for the WebTime analyzer. Just one camera was used for the workload as it was enough to create the model of the system. Figure 9-5 shows a few event-traces of this events log.

\begin{tabular}{|c|c|c|c|c|c|c|c|c|c|}
\hline $\begin{array}{l}\text { Invo- } \\
\text { cation } \\
\text { index }\end{array}$ & $\begin{array}{c}\text { Parent } \\
\text { thread } \\
\text { index }\end{array}$ & $\begin{array}{l}\text { Thread } \\
\text { index }\end{array}$ & $\begin{array}{l}\text { Event } \\
\text { index }\end{array}$ & $\begin{array}{l}\text { event } \\
\text { type }\end{array}$ & $\begin{array}{c}\text { task } \\
\text { name }\end{array}$ & Entry & $\begin{array}{l}\text { CPU } \\
\text { time }\end{array}$ & $\begin{array}{l}\text { Multi- } \\
\text { plicity }\end{array}$ & $\begin{array}{l}\text { CPU } \\
\text { name }\end{array}$ \\
\hline 5 & 0 & 1 & 1 & EX & vidCtrl & 0 & 0 & & \\
\hline 2 & 0 & 1 & 2 & $A C$ & vidCtrl & 0 & 171601.1 & 10 & \\
\hline 1 & 0 & 1 & 2 & $A C$ & vidCtrl & 0 & 171601.1 & 10 & \\
\hline 3 & 0 & 1 & 2 & $A C$ & vidCtrl & 0 & 202801.3 & 10 & \\
\hline 4 & 0 & 1 & 2 & $A C$ & vidCtrl & 0 & 202801.3 & 10 & \\
\hline 5 & 0 & 1 & 2 & $A C$ & vidCtrl & 0 & 171601.1 & 10 & \\
\hline 5 & 0 & 1 & 3 & SE & vidCtrl & 0 & 0 & & \\
\hline 3 & 0 & 1 & 3 & SE & vidCtrl & 0 & 0 & & \\
\hline 1 & 0 & 1 & 3 & SE & vidCtrl & 0 & 0 & & \\
\hline 2 & 0 & 1 & 3 & SE & vidCtrl & 0 & 0 & & \\
\hline 4 & 0 & 1 & 3 & SE & vidCtrl & 0 & 0 & & \\
\hline 2 & 749809 & 1739102 & 31 & $\mathrm{RE}$ & vidCtrl & 0 & 0 & 10 & $\begin{array}{l}\text { Client- } \\
\text { CPU }\end{array}$ \\
\hline 2 & 749809 & 1739102 & 32 & $A C$ & vidCtrl & 0 & 202801.3 & 10 & \\
\hline 3 & 1208111 & 1127265 & 31 & RE & vidCtrl & 0 & 0 & 10 & $\begin{array}{l}\text { Client- } \\
\text { CPU }\end{array}$ \\
\hline 3 & 1208111 & 1127265 & 32 & $A C$ & vidCtrl & 0 & 187201.2 & 10 & \\
\hline 5 & 976032 & 531146 & 31 & RE & vidCtrl & 0 & 0 & 10 & $\begin{array}{l}\text { Client- } \\
\text { CPU }\end{array}$ \\
\hline 4 & 1213997 & 1858556 & 31 & RE & vidCtrl & 0 & 0 & 10 & $\begin{array}{l}\text { Client- } \\
\text { CPU }\end{array}$ \\
\hline 4 & 1213997 & 1858556 & 32 & $A C$ & vidCtrl & 0 & 202801.3 & 10 & \\
\hline 5 & 976032 & 531146 & 32 & $A C$ & vidCtrl & 0 & 171601.1 & 10 & \\
\hline 1 & 430801 & 891917 & 31 & $\mathrm{RE}$ & vidCtrl & 0 & 0 & 10 & $\begin{array}{l}\text { Client- } \\
\text { CPU }\end{array}$ \\
\hline
\end{tabular}

Figure 9-5 a few number of event-traces 
A sub-model was created for each invocation of the "video control". An invocation consisted of capturing a video frame from the camera and storing it in the database. To create a trace file for each invocation, all the event-traces of the recorded events logs of various tasks, of BSS and the database server, were regrouped based on their invocation identifiers and distributed into the trace files of various invocations. Figure 9-6 shows this distribution.

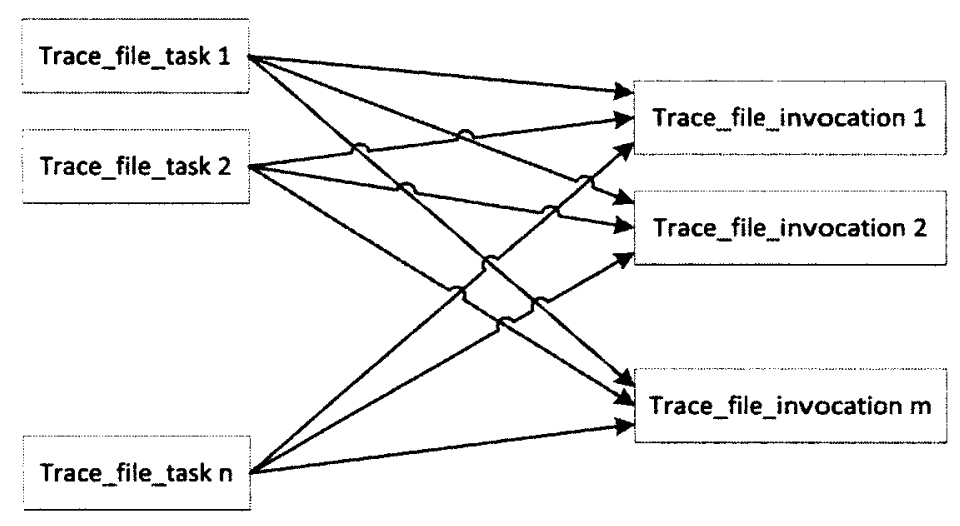

Figure 9-6 distribution of event-traces of tasks to trace files of invocations

For each invocation, a sub-model was created and then all the sub-models were averaged into the final model. Figure 9-7 shows the resulting model. This model was then solved using LQN simulator [65] to obtain the response time of the system for a single camera as well as the utilization level of hardware and software resources of the system. These results are shown in Table 9-2 and Table 9-3 


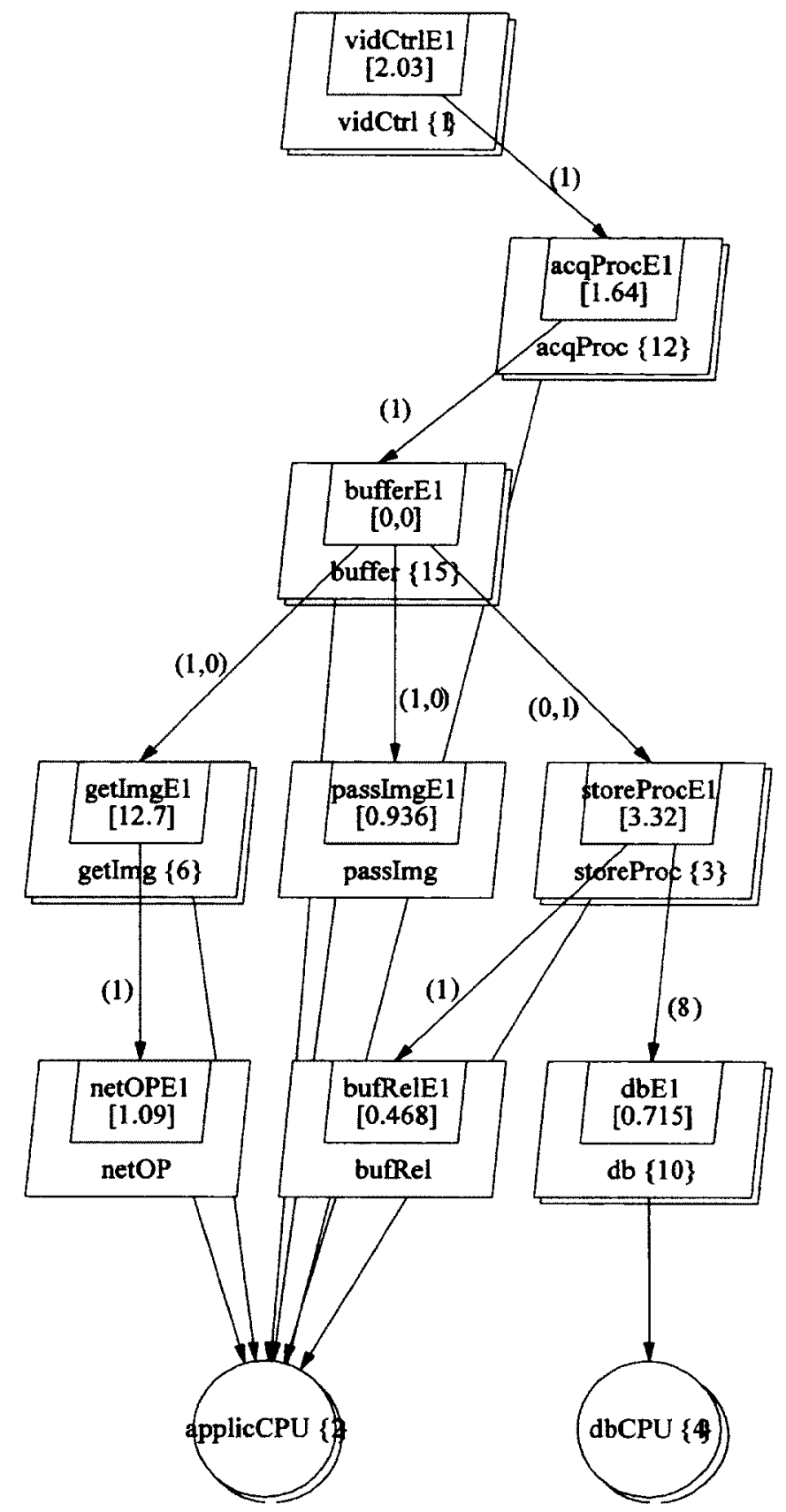

Figure 9-7 the LQN model created for the video control scenario of BSS 
Table 9-2 software and hardware utilization numbers of BSS tasks and CPUs

\begin{tabular}{|l|c|l|}
\hline \multicolumn{1}{|c|}{ Task/CPU } & Threads/CPUs multiplicity & utilization \\
\hline buffer & 15 & 1.3757 \\
\hline $\mathrm{db}$ & 10 & 0.31237 \\
\hline get/mg & 6 & 0.75194 \\
\hline storeProc & 3 & 0.55422 \\
\hline bufRel & 1 & 0.028201 \\
\hline acqProc & 12 & 0.89113 \\
\hline vidCtrl & 1 & 1 \\
\hline netOP & 1 & 0.060332 \\
\hline passlmg & 1 & 0.048793 \\
\hline Application server CPU & 2 & 1.1 \\
\hline Database server CPU & 4 & 0.31 \\
\hline
\end{tabular}

Table 9-3 total averages of response times obtained with measurement and modeling methods

\begin{tabular}{|l|l|}
\hline & Response time \\
\hline BSS (msec) & 33396 \\
\hline LQN model (msec) & 18100 \\
\hline
\end{tabular}

These results show that the response time predicted by WebTime was about $55 \%$ of the measured value. The extracted LQN model was then used to identify the bottlenecks of the system when the number of cameras was increased and also to predict the response times of the system under this condition. The predicted utilization levels of the hardware resources and the tasks of BSS are shown in Table 9-4. As can be seen the model predicted that if the number of cameras was increased to 3 the application server CPU would saturate and Task "acquisition processing" would reach very close to its execution capacity limit. This table also shows that if the number of cameras reaches 5 , Task 
"acquisition processing" will change to a complete software bottleneck and Task "buffer" becomes very close to a software bottleneck. The model predicts that the response times that the cameras would be experiencing would be increased accordingly because of these bottlenecks.

To assess the predictions made by the model, the response time of the system for 3 and 5 cameras were measured and compared with the predicted values of Table 9-5 and Figure 9-8. In all cases, the predicted response times were about $55 \%$ of the real response times. This indicates that although the prediction accuracy of the WebTime for BSS was low, it accurately predicted bottlenecks of the BSS. According to the model, in order to increase the capacity of BSS, one must both increase the number of CPUs of application node and increase the number of software threads in Task "store process".

Table 9-4 software and hardware utilization numbers of BSS tasks

\begin{tabular}{|l|r|r|r|r|}
\hline Task/CPU & $\begin{array}{l}\text { Threads/CPUs } \\
\text { multiplicity }\end{array}$ & $\begin{array}{l}\text { Utilization } \\
\mathbf{( 1} \\
\text { camera })\end{array}$ & $\begin{array}{l}\text { Utilization } \\
\mathbf{3} \\
\text { camera) }\end{array}$ & $\begin{array}{l}\text { Utilization } \\
\mathbf{( 5} \\
\text { camera })\end{array}$ \\
\hline buffer & 15 & 1.3757 & 11.222 & 14.564 \\
\hline db & 10 & 0.31237 & 0.56915 & 0.55744 \\
\hline getImg & 6 & 0.75194 & 1.696 & 1.9126 \\
\hline storeProc & 3 & 0.55422 & 2.8841 & 2.9995 \\
\hline bufRel & 1 & 0.028201 & 0.19012 & 0.19893 \\
\hline acqProc & 12 & 0.89113 & 2.8052 & 4.8083 \\
\hline netOP & 1 & 0.060332 & 0.24345 & 0.31718 \\
\hline passImg & 1 & 0.048793 & 0.16857 & 0.24046 \\
\hline application & 2 & 1.1 & 1.98 & 1.98 \\
\hline database & 4 & 0.31 & 0.56 & 0.56 \\
\hline
\end{tabular}


Table 9-5 total averages of response times obtained with measurement and modeling methods

\begin{tabular}{|l|r|r|r|}
\hline & $\begin{array}{l}\text { Response time } \\
\text { (1 camera) }\end{array}$ & $\begin{array}{l}\text { Response time } \\
\text { (3 camera) }\end{array}$ & $\begin{array}{l}\text { Response time } \\
\text { (5 camera) }\end{array}$ \\
\hline BSS (msec) & 33396 & 57292 & 92116 \\
\hline LQN model (msec) & 18100 & 32440 & 50946 \\
\hline
\end{tabular}

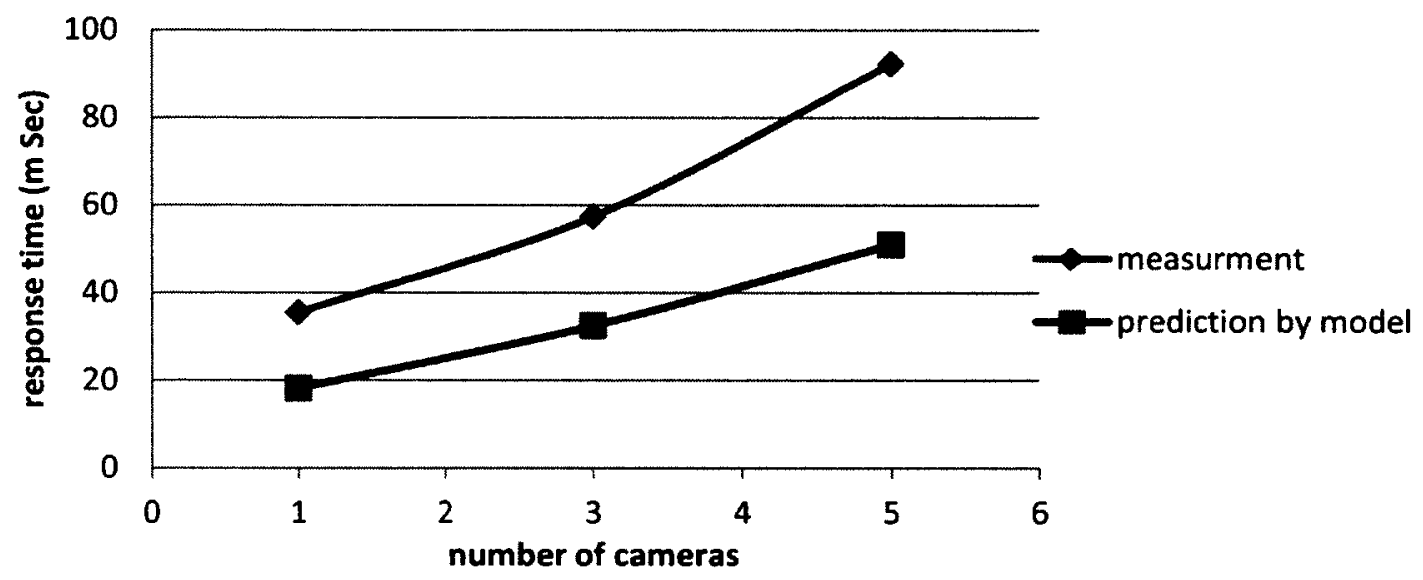

Figure 9-8 response times to cameras versus the number of cameras being serviced

\subsection{Results}

WebTime was used to analyze performance behaviours of a prototype of BSS which was implemented using Java programming in order to identify its software and hardware performance bottlenecks. This prototype was instrumented to generate event-traces when it was running. For each invocation of this system, an LQN performance sub-model was automatically created from the event-traces. These sub-models were then averaged to 
create the overall performance model of BSS. The final LQN model was then used to predict the average response time of BSS for a number of cameras ranging from 1 to 5 . The LQN model was also used to identify the resources of BSS which had reached their maximum execution capacity when the number of cameras was increasing. Finally in order to assess the predictions made by the model, the response times of the BSS prototype was measured by rerunning it for 3 and 5 input cameras.

The hardware and software performance bottleneck of BSS were accurately predicted from the model WebTime created. The accuracy of predicting the response times to a camera was about $55 \%$ of the measured values regardless of the number of cameras. The difference in predicted and measured response times of BSS is mainly attributed to the extent by which the executed events of BSS were covered by the instrumentation mechanism, and also by the accuracy of the methods of Java management factory [66] class which was used to measure the CPU consumption time of the events. 


\section{Chapter 10: RUBBoS Case study}

This case study is to compare the measured performance metrics of a real distributed application with those which were predicted by the model which WebTime automatically created. This comparison will be used as an evaluation of the representativeness of the model which WebTime creates and discuss how it can be improved.

\subsection{RUBBoS}

RUBBoS [16], which is an open source benchmarking tool for bulletin board web applications, was chosen to investigate its performance behaviours using WebTime. RUBBoS was created to generate performance benchmark data such as memory, hard drive and CPU usage for a bulletin board web application and has extensively been used for research and studying web and n-tier applications [76][77][78][79][80][81]. In essence, RUBBoS consists of an emulated web browser which generates traffic, plus an online news forum webserver which tries to emulate Slashdot [82]. RUBBoS uses a three-tier version of the Java Enterprise Edition [83] architecture, as shown in Figure 10-1. The first block in this figure, the emulated web browsers, is a Java program which emulates a number of clients which access the webserver. This program creates and sends various and randomly selected uniform resource locators (URLs) to the webserver. These 
URLs are created by a state transition mechanism driven by a pre-loaded probability table. The webserver is constructed with Java Servlet technology[84].

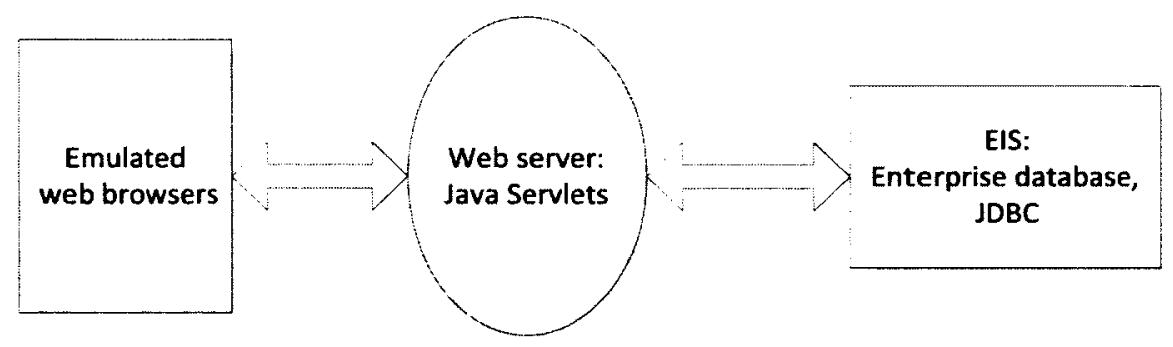

Figure 10-1 A three-tier Java Enterprise Edition

Generally servlets are the main component of a Java based web application. They are programs which run on a webserver and build web pages on the fly.. To deploy and run a servlet, a web container such as Apache Tomcat [85] must be used. The web container is responsible for managing the lifecycle of servlets, mapping a URL to a particular servlet and ensuring that the URL requester has the correct access rights. A Java web application can get an arbitrary name, which would be the root of the directory in which the web applications files are placed. All the files, as well as the sub-directories underneath a root directory, are accessible in a web application except a directory named WEB_INF. Inside WEB-INF there are two important directories, classes and lib, and one important file, web.xml. The class directory contains all the compiled Java classes which are part of a web application. The lib directory contains all the JAR files used by a web application. The web.xml file contains information about the web application, which is used by the container in order to properly deploy and execute the web application. For instance, 
web.xml contains information about all the servlets which a web application should deploy and the mapping between received URLs and the deployed servlets.

The database server of RUBBoS runs a MySQL [86] program which is invoked by the Apache Tomcat container and remotely accessed through JDBC [87] technology.

The component of RUBBoS which is used for extracting benchmark values is the emulated web browser, whose architecture is shown in Figure 10-2. This figure shows the objects and their interactions in the client node. The "clientEmulator" object creates user sessions, collects statistics from the webserver and the database server, and creates a final performance report. First, the emulated web browser connects to the database server and the webserver to prepare specific locations for data logging. Next, it creates the user session threads in which the responsibility of interacting with the webserver is delegated. These threads collect statistics such as the number of all the interactions happened in a test run, the total duration of the entire user sessions, and the count and the average duration of each user session. When all the interactions are complete, the clientEmulator object connects to the webserver and the database server to collect the information which these servers have logged during the system operation in order to create the final report. 


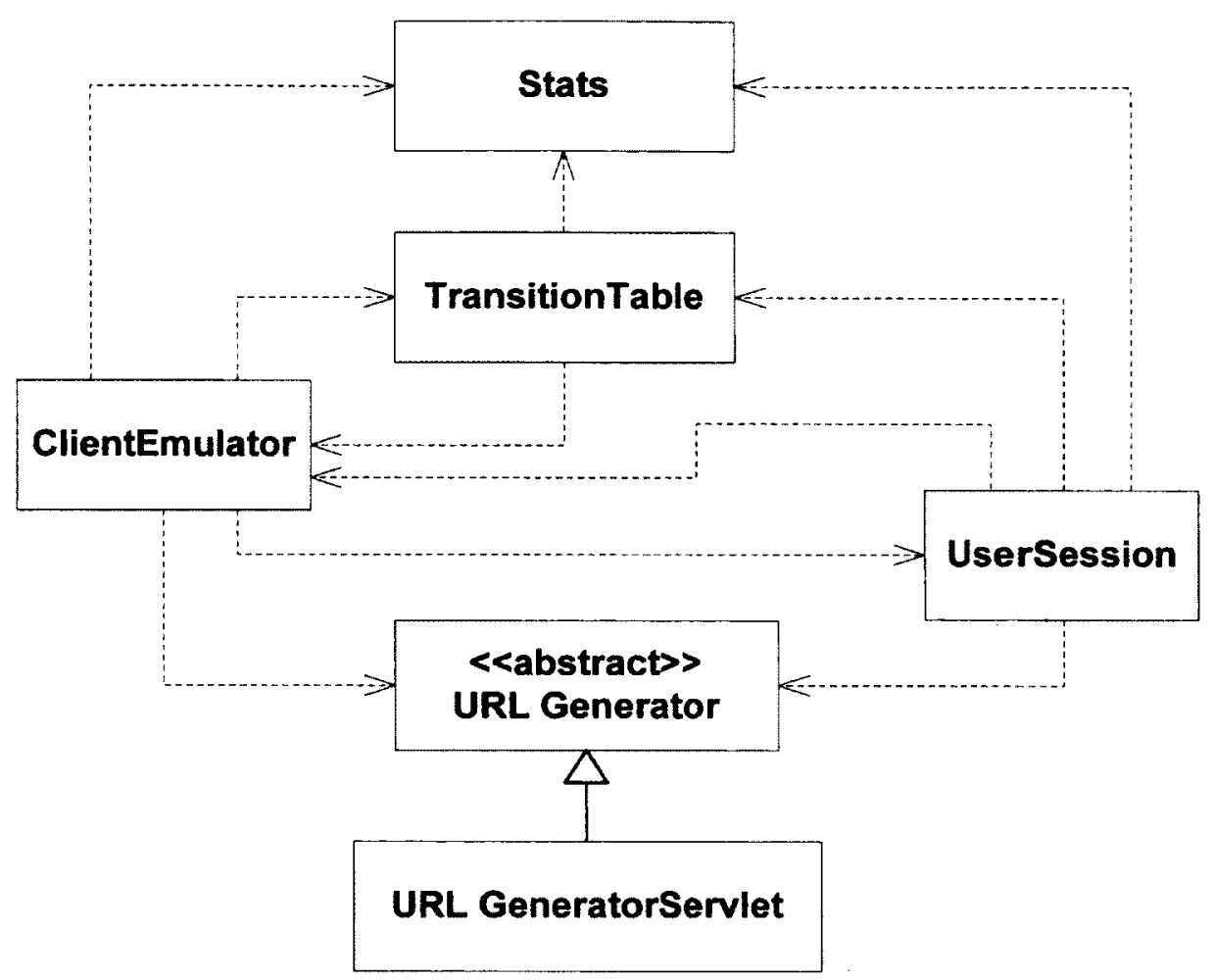

Figure 10-2 architecture of the emulated web browser

The concept of a "user session" term, which is used several times throughout this chapter, is explained here. As shown in Figure 10-3, a user session is a consecutive series of interactions which a user web browser makes during its visit to the RUBBoS webserver. These interactions are made by sending various URLs to invoke the various services provided by the servlets in the webserver. 


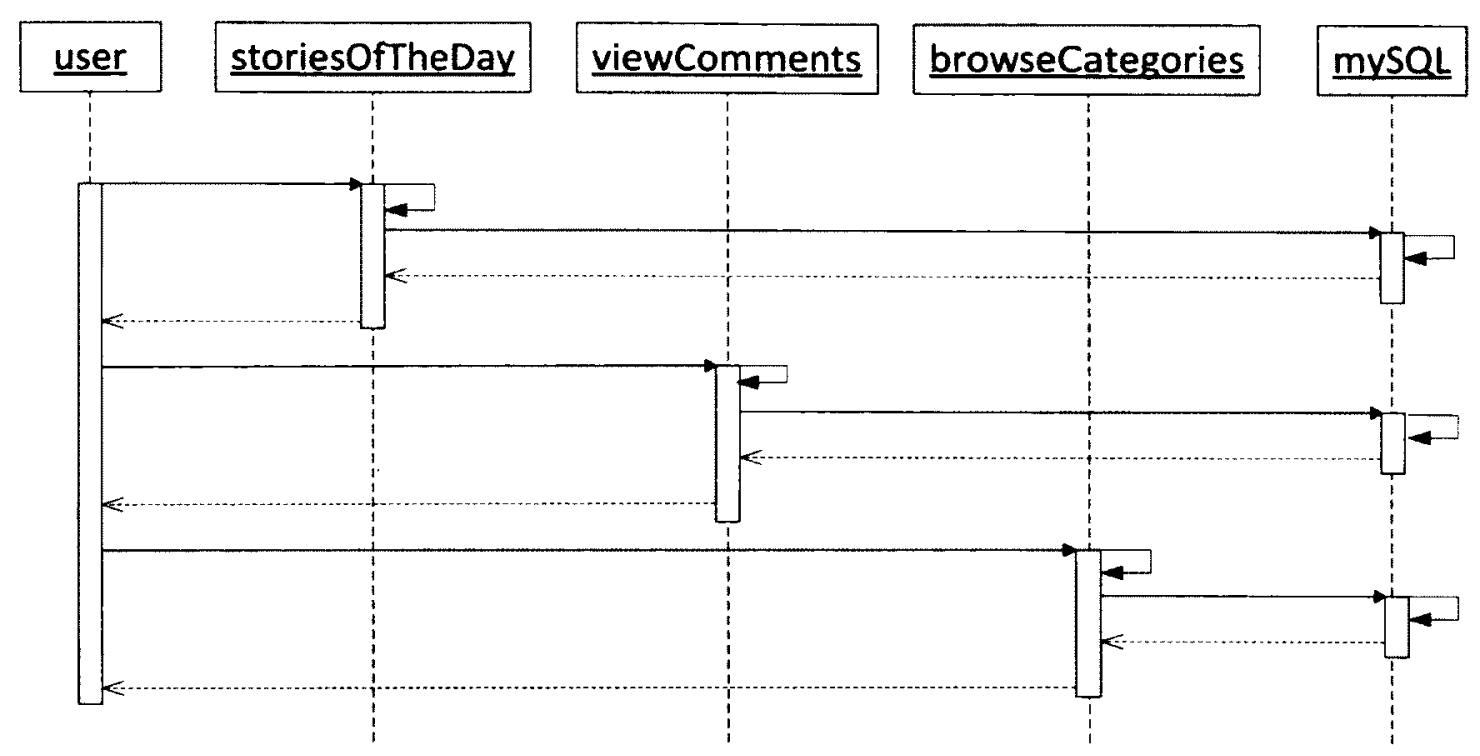

Figure 10-3 examplae of a user session

Figure 10-4 shows the architecture of this webserver. The webserver in RUBBoS uses the Apache-Tomcat container. This container contains various servlets to cover the expected services of a bulletin board application. The names of these servlets are listed in Table 10-1. The servlet classes in this figure implement the "HttpServlet" class [84]. Each server class is associated with a configuration class through which the necessary parameters for connecting a servlet to the database server, such as user name, password, and database name can be set. 


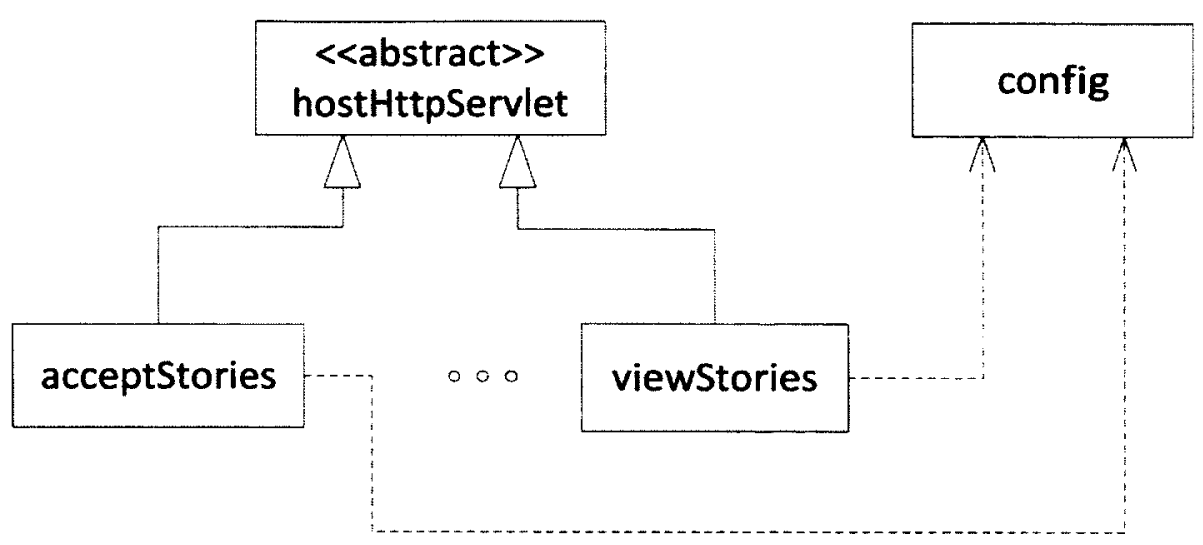

Figure 10-4 architecture of the webserver

Table 10-1 mapping servlets of RUBBoS to entry identifiers of an LQN model

\begin{tabular}{|l|c|}
\hline Servlet & Entry \\
\hline Accept stories & 1 \\
\hline Author & 2 \\
\hline Brows categories & 3 \\
\hline Brows stories by category & 4 \\
\hline Moderate comment & 5 \\
\hline Older stories & 6 \\
\hline Post comments & 7 \\
\hline Register user & 8 \\
\hline Reject stories & 9 \\
\hline Review stories & 10 \\
\hline search & 11 \\
\hline Store comments & 12 \\
\hline Store moderator comments & 13 \\
\hline Store story & 14 \\
\hline Stories of the day & 15 \\
\hline Submit story & 16 \\
\hline View comments & 17 \\
\hline View story & \\
\hline
\end{tabular}




\subsection{Messaging}

RUBBoS uses the Java URLConnection [88] class for sending messages from the clientEmulator to the webserver. Class URLConnection is the superclass of all classes that represent a communications link between an application and a URL object [89]. An URLConnection object can be extracted from a URL object using the URL's "openConnection" method. The URLConnection object can be used both to read from and to write to the resources referenced by a URL object. When the clientEmulator wants to communicate with a servlet, it creates a URL for that servlet and extracts the URL's URLConnection. Then, the client emulator streams its HTML file to that URLConnection.

Sending a message from the webserver to the clientEmulator is performed using the Java HttpServlet class. The servlet container creates a HttpServletRequest [84] and a HttpServletResponse [84] objects from a request that it receives and passes them as arguments to the servlet's service methods doGet() and doPost(). Receiving a message from the clientEmulator consists of receiving a stream of HTML strings from the HttpServletRequest. Sending a reply message to the client emulator consists of streaming a HTML message to the HttpServletResponse object.

Timestamp objects of WebTime were transferred along with the messages exchanged between the clientEmulator and the webserver. When a HTML message is about to be sent, the timestamp object is converted to a HTML string and streamed along with the message. When a HTML message is received, the part of the message which is related to the timestamp is extracted and converted into a timestamp abject. 


\subsection{Experimental setup}

Figure 10-5 shows the deployment of RUBBoS in this work. The specifications of each node are as follows:

- The client side web browser emulator is deployed in a separate computer with Intel Core2 Duo running at $2.1 \mathrm{GHz}$ using the Linux Ubuntu as operating system.

- The webserver node is a computer with Intel core i7 running at $2.6 \mathrm{GHz}$ using the Linux Ubuntu operating system. This node and the client node are located on the same LAN.

- The database node is a computer with Intel i7core running at $2.6 \mathrm{GHz}$ using Linux CentOs operating system. This computer is connected to the webserver through a WAN network.

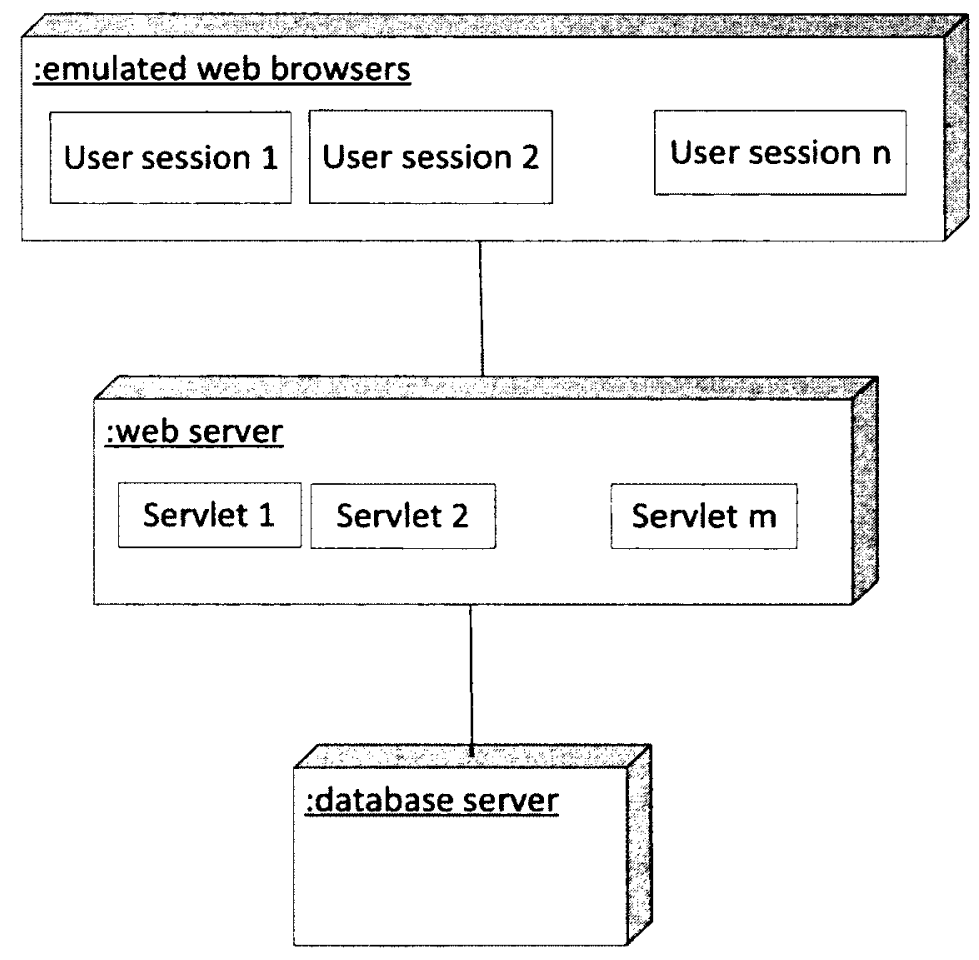

Figure 10-5 deployment diagram of RUBBoS 


\subsection{Monitoring system}

In order to generate and collect event-traces, a monitoring system was created and added into the RUBBoS benchmark. This system consists of a logging system for each node. Each logging system consists of a logger thread and a trace-queue, shown in Figure 10-6. The reference of the trace-queue in each node is passed to all the objects of that node which generate traces. The trace-queue is a FIFO queue of blocking type to prevent simultaneous access by various thread objects. The logger thread frequently checks the contents of its associated trace-queue and transfers events, if any, to a trace file in the file system of that node. To analyze the performance of a system after a test run, the trace files from all the nodes were used in order to create the final LQN model of the system.

The way in which the logger thread and its associated trace-queue are implemented in a node depends upon the type of the node, as it is explained below. The clientEmulator object creates the trace-queue and also spawns the logger thread of its node before spawning its user session threads. These user session threads are used by the clientEmulator object to send its service requests to the webserver. The clientEmulator passes the reference of its node trace-queue to all the user session threads it spawns. The original user session thread class of RUBBoS was modified in order to add the tracequeue reference into its input argument. In this way, the user sessions were allowed to put their event-traces into the trace-queue of the client node as they run. 


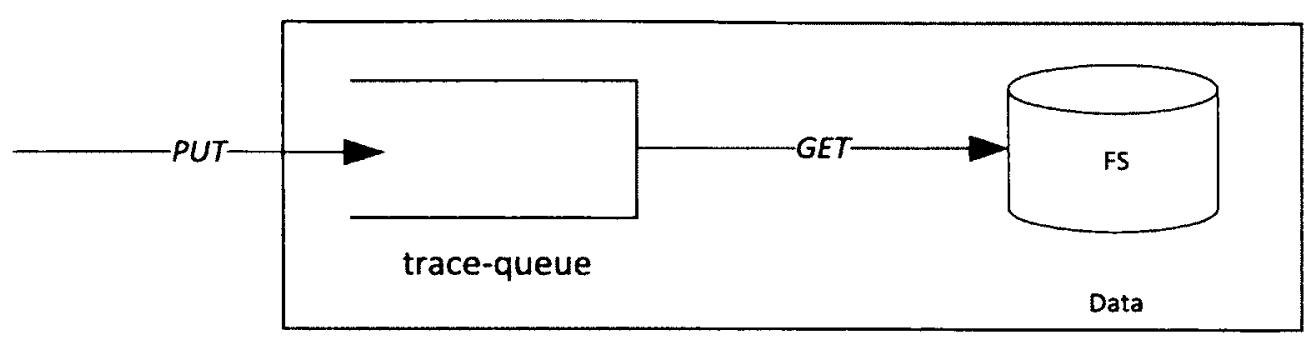

Figure 10-6 the logger thread

Monitoring at the server side is more involved since the servlets are under control of Apache Tomcat. To manage this situation, a new servlet is created, called "start up", which is responsible to create the trace-queue and also to spawn the logger thread of the webserver node. This servlet performs these operations as soon as it is loaded. One has to make sure that this servlet is initialized before all the other servlets of the container. This can be done by setting an element in web.xml file called $<$ load-on-startup $>$.

The original RUBBoS servlets of the container were modified in order to accept a reference to the trace-queue. For this, it was required that the servlets of the webserver communicate with each other in order to pass the trace-queue reference. One way the servlets of a container can communicate with each other is through an object called "servletConfig" [84]. This object contains any initialization parameter and start up configuration for a servlet. The "start up" object adds the reference of the trace-queue of the webserver node to this object. The other servlets were also modified to get this reference from the "servletConfig" object when they are invoked. In this way, all the 
servlets of a container were able to send their event-traces to the trace-queue of the webserver node.

\subsection{Instrumentation}

Instrumentation code was added to RUBBoS to capture three important event types:

- Send a message,

- Receive a messages, and

- CPU-time consuming activities.

The instrumentation code were methods which were added where the send, receive and CPU consuming activities of RUBBoS took place. The instrumentation methods were incorporated into a Java class called "Instrumentation", which was added into the RUBBoS project. The instrumentation methods were:

- $\operatorname{send}()$

- $\operatorname{sendAck}()$

- receive()

- process()

- external()

Figure 10-7 shows the locations in a program where these methods were inserted. It should be noted that the database server was not instrumented and, instead, its instrumentation was performed at the webserver side. 


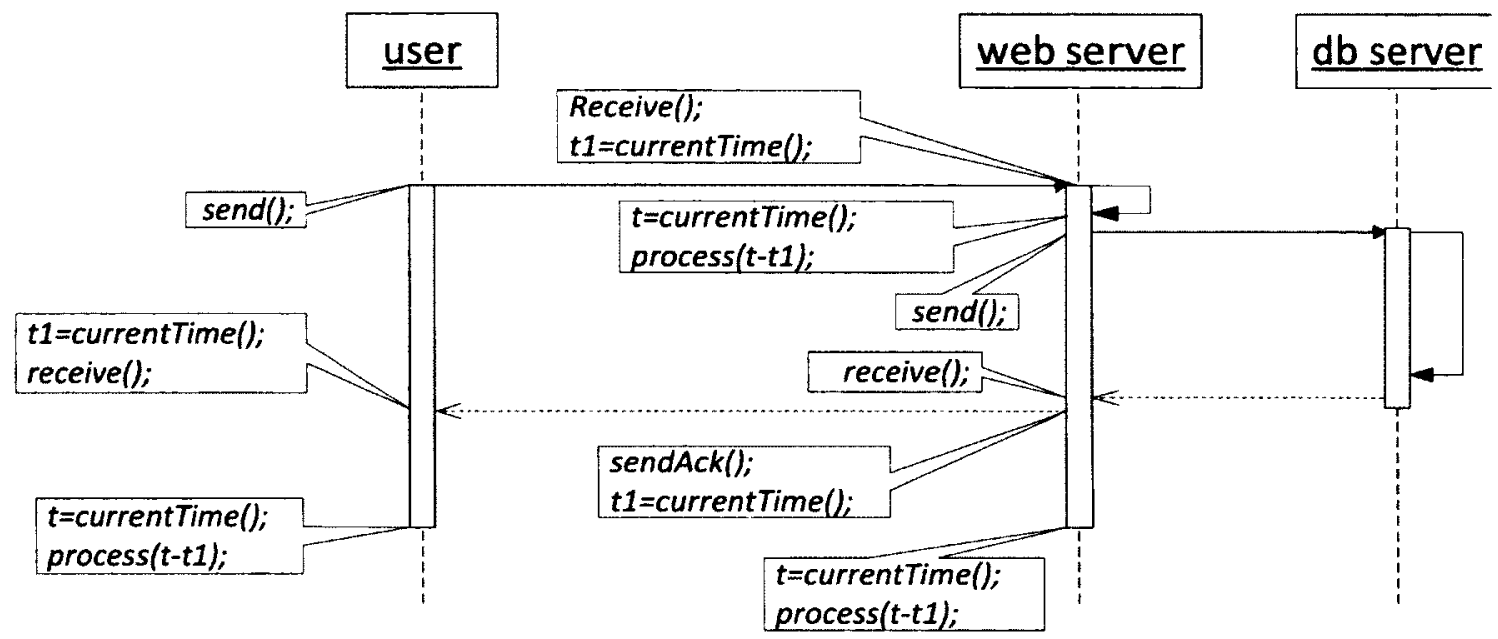

Figure 10-7 the locations in a program where instrumentation methods are added

The send() method was inserted before a real send message operation in RUBBoS. Two types of send messages were identified:

1. HTML messages which were sent from the clientEmulator to the webserver,

2. Database queries which were sent from the webserver to the database server.

In the client emulator, the send() method was inserted after a timestamp object was processed for sending to the webserver and before a HTML message was streamed through the URLConnection object of the destination servlet. In the webserver, the send() method was inserted before sending a query to the database server.

The sendAck() method was inserted before an actual response message code. Two types of response messages were identified in RUBBoS:

1. A response from the webserver to the clientEmulator 
2. A response from database server to the webserver

In the webserver, the sendAck() method was inserted after a message was streamed to the HttpServletResponse object which was passed to the webserver's doPost().

The sendAck() of the database server was added at the webserver right after receiving a query result from the database server, pretending it was performed at the database server side.

The receive() method was inserted right after an actual reception of a HTML stream. Three types of receive messages were identified in RUBBoS:

1. Reception of a HTML message from the clientEmulator at the webserver

2. Reception of HTML message from the webserver at the clientEmulator

3. Reception of query response from the database server at the webserver

At the webserver, and the clientEmulator, the receive() method was inserted right after the timestamp object was recovered from the received HTML stream. For the database server, the receive() method was inserted at the webserver when the response to a query was received.

The process ( method was used to record the CPU consumption time of a node. Basically, it was assumed that the following intervals have direct relationship with the processing time of a CPU.

- In a servlet, between receiving a request and sending a request to the database server,

- In a servlet, between receiving an acknowledge message from the database server and sending an acknowledgement to a client, 
- In the database server, between receiving a request and sending an acknowledge to it,

- In a client between receiving an external trigger and sending a request to the webserver,

- In a client between receiving an acknowledgement and the end of that client thread.

To measure a processing interval, a time marker was placed at the beginning of the interval, and the process() method was inserted at the end of this interval, as shown in Figure 10-7. The time difference between these two events was passed to the process() method. The processing time of a query in the database server was measured at the webserver. Obviously, this time interval included the sending and receiving time of a query message too, which should be considered when the results are analyzed.

The Java Management Factory class [66] was used to measure the time intervals between the mentioned events. This class provides support for reporting CPU and user time per thread while avoiding other system activities.

The messages in RUBBoS carry the WebTime's timestamp objects. The four key components of this timestamp, which allow for causal ordering of the recorded traces are:

1. Invocation (session) identifier

2. Current thread identifier

3. Parent thread identifier

4. Event identifier 
In this particular application, all the interactions between the client and the webserver, as well as the webserver and the database server, are of blocking (synchronous) type. This allows these parameters to be reduced to:

1. Invocation (session) identifier

2. Event identifier

The following local parameters were also added to the event-trace structure of WebTime:

1. Task identifier (clientEmulator, webserver, database server).

2. Task multiplicity (thread count).

3. Entry (servlet) identifier.

4. Host identifier (CPU or MAC address).

5. Event type (“SE", “RE”, “AC”, “EX”, "SA").

6. CPU-time.

For each servlet an identifier was specified to represent it as an "entry" in the final LQN model. Table 10-1 shows the entry identifiers used for the servlets of this application. The MAC address of each host was extracted from the "/proc/net/arp" file to represent the host's identifier. The CPU multiplicity was also extracted from the "/proc/cpuinfo" file. The task multiplicity is a parameter specified by the container. The minimum and maximum for this value is set in the "server.xml" file of Apache Tomcat (located in the "/opt/apache-tomcat/conf" folder). 
The type of an event can be determined by the location where instrumentation methods were inserted. Table 10-2 shows the correspondence between the instrumentation method and the event type recorded.

Table 10-2 Instrumentation methods and their correspondence with events types

\begin{tabular}{|l|l|c|}
\hline $\begin{array}{l}\text { Instrumentation } \\
\text { method }\end{array}$ & Event type & $\begin{array}{l}\text { Acronym for event } \\
\text { type }\end{array}$ \\
\hline send() & Service request & "SE" \\
\hline sendAck() & Service result send & "SA" \\
\hline receive() & Receive a request & "RE" \\
\hline process() & Process activity & "AC" \\
\hline external() & External invocation trigger & "EX" \\
\hline
\end{tabular}

The instrumentation code insertion process started by the creation of a timestamp object in the "clientEmulator" class called "initial timestamp". The "external trigger" method is also placed in the "clientEmulator" class before initiation of any "user session" threads in this class. Each "user session" used a new timestamp object with a different invocation number. These timestamp objects were created from the initial timestamp object by cloning the initial timestamp. To get a new invocation number for the timestamp object of a new user session, the invocation number of the initial timestamp was incremented and then cloned. The reference of a new timestamp was passed to the user session thread for which the timestamp was created. Therefore, a timestamp object traversed through the webserver, the database, back to the webserver and then back to the client, when the system was running. 
The webserver in the original form of RUBBoS responds to a HTTP request by executing a "doGet()" method. Technically this method only forwards a static HTML file back to the clientEmulator. This was not enough for this case study as the clientEmulator also needed to receive a different timestamp in response to the timestamp it sends to a servlet. In other words, the received timestamp needed to be processed at the destination servlet and the modified timestamp passed by the response message to the client. The solution was to use "doPost()" methods instead of "doGet()" ones. A "doPost()" method allows performing processing on the received request and creating a custom HTML file according to the received request. The "doPost()" method in this case study also needed to perform the original functionality which was provided by the "doGet()" methods. Therefore, the "doPost()" method in each servlet, in addition to processing the received timestamp, called the original "doGet()" method of that servlet.

The extra processing load of a servlet due to insertion of instrumentation code consisted of the followings:

1. decoding the part of the message containing the timestamp information (after receiving a HTTP request),

2. performing the receive() function of the instrumentation object,

3. processing of the timestamp,

4. performing the process() function of the instrumentation object,

5. coding the resulting timestamp into HTML format,

6. performing the send() function of the instrumentation object (and sending the HTML response back to the client) 
The average number of lines of a servlet in RUBBoS is about 500. The total extra number of lines added for performing the above points was about 50. Therefore, the processing overhead due to instrumenting RUBBoS was about $10 \%$ on average.

\subsection{Experiments}

A set of experiments were performed to assess the results obtained from the application of WebTime. The first experiment was performed to assess the accuracy of the predicted metrics obtained using WebTime with respect to the measured metrics using RUBBoS. An LQN performance model was created from this experiment. This model was used to predict the performance behaviour of the system under various environmental conditions such as the numbers of users and the set up for the hardware and software resources.

In order to facilitate the analysis of the results, it was decided to get the client emulator to generate almost identical user sessions through all the experiments. There were two parameters which made the user sessions in an experiment analogous: the sequence of interactions from a user to the various servlets in the webserver and the thinking period of a user before each interaction. Therefore, the configuration of RUBBoS was changed to work with an almost identical workload, and a constant thinking time period before an interaction. Setting this configuration was straightforward and was done by modification of the pre-loaded probability table.

To create the LQN model of the system, it was run under the lightest workload using only one user with the system. In this condition the likelihood of having a bottleneck, either hardware or software, was minimal. It was assumed that, under this condition, a 
more accurate model would be achieved. The user made interactions with the webserver using a running duration of approximately $150 \mathrm{sec}$, during which about 92 user sessions were performed. RUBBoS internally measured the duration of each user session, as well as the average duration of all the user sessions. When the system was running, it generated events traces which were used to construct the performance model of each user session, called sub-models. These sub-models were then combined to create the final LQN models of the system. Figure 10-8 shows this model.

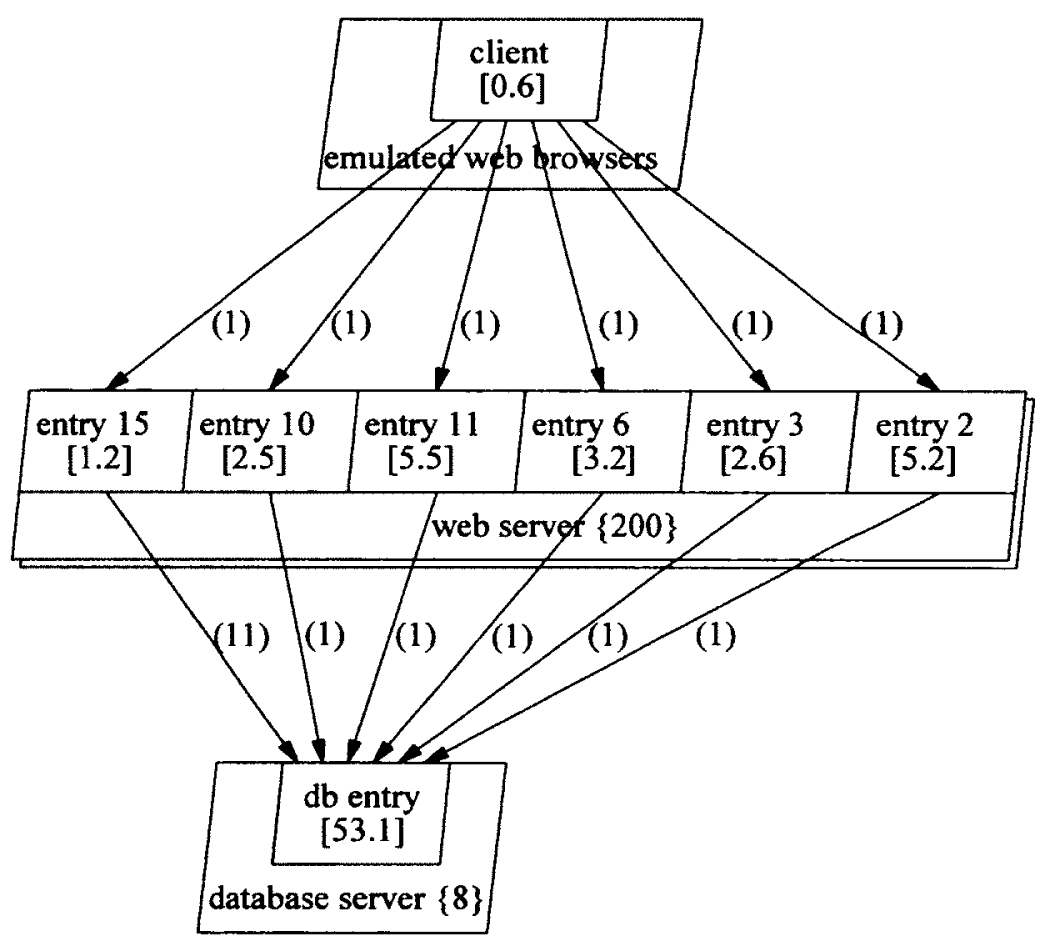

Figure 10-8 the overall LQN model of the first experiment 
The average user session durations obtained from the model and from RUBBoS are shown in Table 10-3. The confidence interval of the average measured values was \pm 19 for a confidence level of $95 \%$.

Table 10-3 total averages of session periods obtained using measurement and modeling methods of experiment 1

\begin{tabular}{|l|l|}
\hline & Average session period \\
\hline RUBBoS(msec) & $4008( \pm 19$ for confidence level of $95 \%$ \\
\hline LQN model & 3114 \\
\hline
\end{tabular}

The utilization levels of the software resources, such as the threads of the tasks, as well as the hardware resources such as CPUs are shown in Table 10-4. A utilization number is a value between " 0 " and " 1 ", representing a not utilized and a fully utilized resource, respectively. This table shows that none of the resources were saturated with the load imposed by one user. The LQN model obtained from this experiment was then used to determine the load at which a resource would saturate and identify that resource. Figure 10-9 shows the average session periods obtained from the LQN model using different numbers of users. This figure clearly indicates that one or more resources would saturate when the number of users reaches about 30 . 


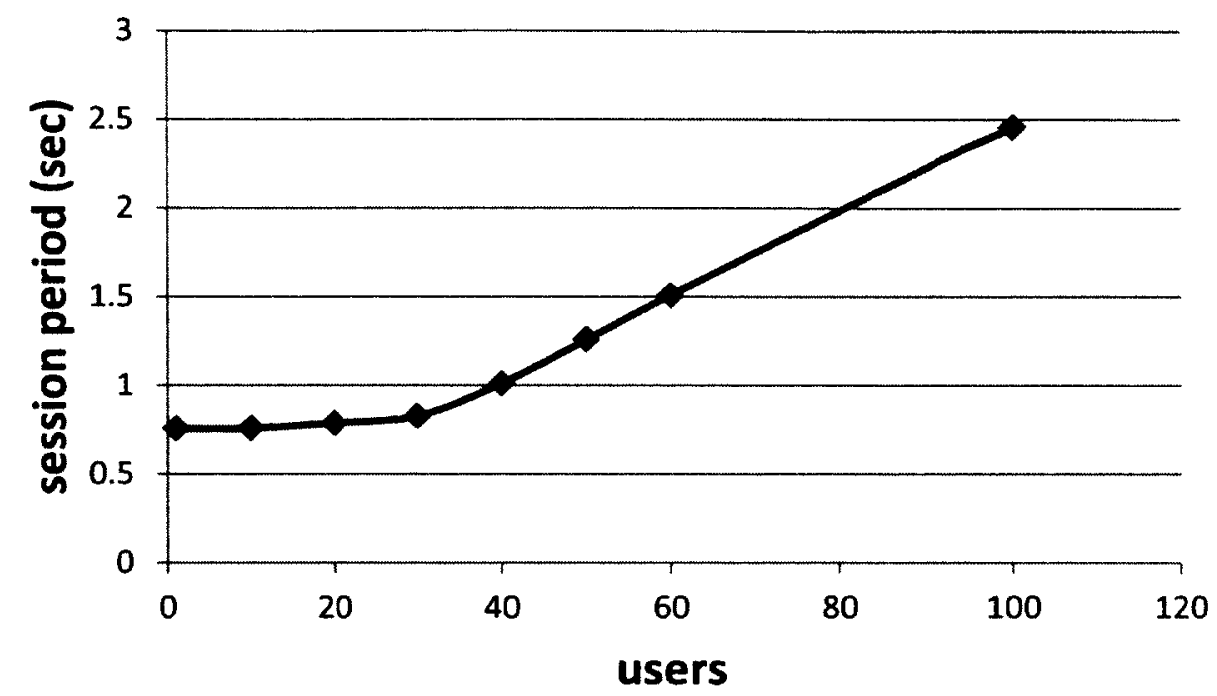

Figure 10-9 predicted session periods versus numbers of users

To identify the saturated resources, the utilization numbers of the various resources of RUBBoS for different numbers of users are shown in Table 10-4. As can be seen, the database server shows clear signs of saturation. First, its CPU saturates when the number of users reaches to 40 . Then, its software threads saturates when the number of users reaches 60.

Table 10-4 software and hardware utilization numbers of the RUBBoS resources

\begin{tabular}{|c|c|c|c|c|}
\hline $\begin{array}{c}\text { Number } \\
\text { of users }\end{array}$ & $\begin{array}{c}\text { Database server } \\
(8 \text { threads })\end{array}$ & $\begin{array}{c}\text { Webserver } \\
(200 \text { threads })\end{array}$ & $\begin{array}{c}\text { Database server } \\
(2 \text { processor })\end{array}$ & $\begin{array}{c}\text { Webserver } \\
\text { (8 processor })\end{array}$ \\
\hline 1 & 0.064 & 0.090 & 0.064 & 0.025 \\
\hline 10 & 0.66 & 0.92 & 0.64 & 0.25 \\
\hline 20 & 1.67 & 2.17 & 1.68 & 0.49 \\
\hline 30 & 4.26 & 5.02 & 1.76 & 0.70 \\
\hline 40 & 7.2 & 12.79 & 1.92 & 0.77 \\
\hline 50 & 7.8 & 22.6 & 1.93 & 0.77 \\
\hline 60 & 7.92 & 33 & 1.93 & 1.54 \\
\hline
\end{tabular}


In the next step RUBBoS was run to investigate the real behaviour of the system for various numbers of users and assess the predicted values. Table 10-5 and Figure 10-10 show the average users session periods obtained from WebTime and RUBBoS for various numbers of users. Overall the predicted values for session periods are $25 \%$ to $30 \%$ shorter than the real values provided by RUBBoS. This is an expected result for the following reasons.

- The instrumentation mechanism used didn't capture the operating system events,

- The duration in which a message was transferred was not captured nor was incorporated into the final performance model,

- The accuracy of the Java management factor for measuring the net CPU-times of events is not validated.

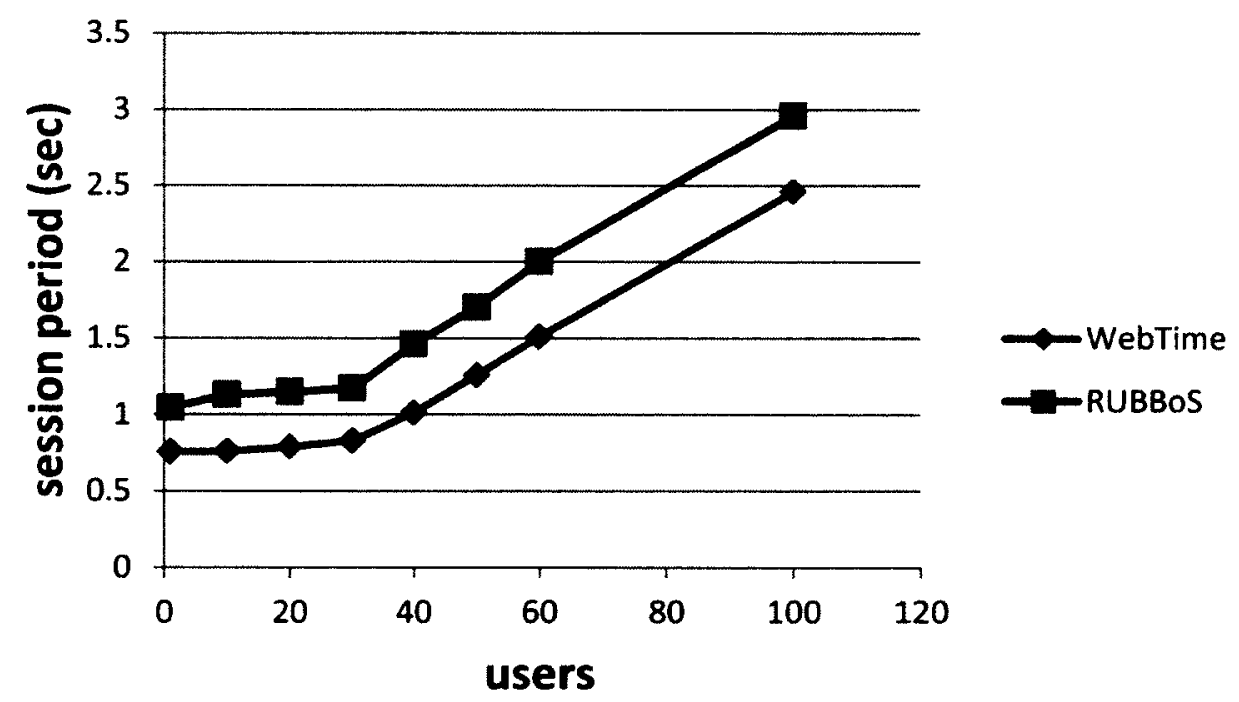

Figure 10-10 user session periods versus numbers of users 
Table 10-5 user session periods for various number of users

\begin{tabular}{|c|c|c|c|c|c|c|c|c|}
\hline users & 1 & 10 & 20 & 30 & 40 & 50 & 60 & 100 \\
\hline RUBBoS (msec) & 1042 & 1128 & 1148 & 1170 & 1459 & 1700 & 2000 & 2956 \\
\hline WebTime (msec) & 755 & 757 & 787 & 826 & 1010 & 1256 & 1507 & 2456 \\
\hline
\end{tabular}

\subsection{Results}

WebTime was used to predict the performance behaviours of RUBBoS and to identify its software and hardware performance bottlenecks. For each user session an LQN performance sub-model was automatically created from the events log generated by RUBBoS while it was running. These sub-models were combined to create the overall performance model of RUBBoS. The final LQN model was then used to predict the average user session period. The LQN model was also used to identify the resources of RUBBoS which had reached their maximum execution capacity when the number of users was being increased. RUBBoS was run several times to obtain the average user session periods in the same condition in which WebTime had predicted them. The prediction accuracy of WebTime was within $70-80 \%$ of the measured values. This difference is mainly attributed to the extent of coverage of the executed events by the instrumentation mechanism and also the accuracy of the method used to measure the CPU consumption time of the events.

WebTime accurately identified the performance bottlenecks of RUBBoS from the first model it created. The results showed that the difference in the predicted and 
measured average session periods does not vary greatly with the increase of workload. Therefore, this difference doesn't affect the capability of WebTime to identify the performance bottlenecks.

The complexity of using WebTime to generate and analyze a model is roughly constant over the number of consumers used to generate traffic to RUBBoS. This contrasts with the linear complexity for obtaining the same result through measurements. Running one iteration of RUBBoS took approximately 20 minutes. Running WebTime to convert the events log into a model took roughly 4 minutes and solving the model took a few seconds. From the results shown here, five benchmark runs of RUBBoS were required to locate the bottleneck throughput at 40 users. From this experiment, WebTime is four times faster. If substantially more runs were needed to find the bottlenecks, the time saving from using WebTime becomes even more substantial.

The analysis of events logs by WebTime scales reasonably. Analyzing a trace consisting of over one hundred thousand events took less than four minutes on a standard lap top computer. This events log consisted of 100 invocation logs. Therefore, creation of each sub-model took less than 2.4 seconds. If the number of invocations were 1000 (10 times of 100 invocations) the total analysis time would have been 40 minutes (10 times of 4 minutes).

Finally, WebTime can be used to find the customer population at which the system is saturated. This number can be used as a starting point for measurement runs, thus reducing the total number of runs required. 
The LQN model created by WebTime was used to determine the utilization of the CPUs and tasks of RUBBoS. These utilizations identified the bottlenecks in RUBBoS. The analyst can then recommend changes to improve performance if necessary. 


\section{Chapter 11: Conclusion and future directions}

This research has presented WebTime which is a framework of tools and methodologies for automatically constructing the LQN performance model of a message passing distributed application based on the client-server paradigm. The model is constructed from the dynamic behaviours of the application using its traces of events and it is accurate enough to provide valuable predictions about the performance metrics of that application.

The amount of time and resources WebTime needs to produce a model and predict the performance of a deployed distributed system can be far smaller than when a measurement approach was adopted instead. WebTime only needs the events $\log$, which can be generated in a reasonable time frame to automatically construct its performance model. This model can be solved using the LQN solver and simulator [65] to predict performance metrics such as the response time and the utilization of the hardware, and the software resources of that system. The same model can also be used to investigate conditions under which the performance of this system can be improved, such as the amount of extra processing capacity to be added to the resources which are, or close to being, performance bottlenecks.

WebTime can also be used in the development cycle of distributed software systems using the SPE methodology. In each evolution of the system, increasing details are 
absorbed by the model and examined to provide feedback on the direction in which the design is going. The same model helps the designers to find the best design options.

WebTime has been evolving and its specifications have been extended beyond those described in [90][91][92][93]. In its current state, WebTime is capable of capturing the dynamic behaviours of a distributed system, automatically extracting and parameterizing its architecture and constructing its LQN performance model with a reasonable computational complexity and accuracy. The reliability of WebTime was validated by applying it to three case studies: 1) BSS simulated using Parasol, 2) the same BSS, but written using Java socket technology and executed on multiple computers, and on 3) RUBBoS, a distributed benchmark of bulletin board web applications written in Java but using Servlet technology on Apache Tomcat.

\subsection{Contributions of WebTime}

WebTime presents a new structure for the timestamp by which the events of a computation are annotated. This timestamp is independent from the wall clock time and in fact is a new causal logical clock. This logical clock property allows using WebTime to model a distributed message passing application without relying on a clock synchronization system. This structure is fixed in size with a very limited number of parameters. This approach is different from that of Vector-Time [14] [15], because the number of parameters grows with the number of tasks in Vector-Time. Furthermore, the WebTime timestamp has smaller number of parameters than that of Proper-Time [13]. Therefore, the timestamp structure of WebTime, inherently by design, imposes the least overhead on the messages passed between the tasks. 
The timestamp structure of WebTime fully supports concurrent operations in a task. This property is another advantage of WebTime over Proper-Time and Vector-Time. The timestamp structure of Proper-Time, despite its complexity, is not sufficient to account for concurrent operations in a task. Vector-Time has a message overtaking problem [43] which prevents it from capturing concurrent operations in a task.

WebTime performs a post mortem analysis which enables it to extract the architectural structure of a computing system from its events log. This analysis is formal and it allows for the automated transformation of the events log into an LQN model. WebTime can capture blocking and non-blocking interactions between the tasks of a computation.

WebTime automatically determines the service times of the tasks from the events log. These service times include the CPU consumption times of the entries plus their demands for services at other entries, which are needed to construct the LQN model. The service time determination is a unique feature of WebTime. Vector-Time and Proper-Time only extract architectural information of a computation and they require that these parameters be manually provided.

The model construction from the events $\log$ of WebTime has a mixed linear and quadratic computational complexity in which the share of quadratic complexity reduces when the number of invocations in the events logs increases. An events log is partitioned into separate invocations, where each invocation is a single interaction by a client. Each invocation is analyzed separately to form a sub-model. The average of all sub-models is used to construct the final model. The analysis of an invocation has $n^{2}$ complexity where 
$n$ is the number of events in an invocation. The averaging of sub-models has linear complexity.

WebTime was tested on a real distributed application called RUBBoS. RUBBoS is a bulletin board benchmark for web applications, written using Java servlet technology. RUBBoS was instrumented to intercept messages, add timestamps and log traces of WebTime events. The instrumentation system was mainly implemented in a small number of Java methods which were mechanically inserted in small number of places in RUBBoS servlets source code. The total number of lines of added instrumentation in each servlet was under 50; the average size of a servlet of RUBBoS was 500 lines. The LQN model created from the post mortem analysis of the events log clearly showed the software and hardware bottlenecks of RUBBoS and predicted the service time and throughput of this system with reasonable accuracy. The computational complexity of the model construction process was close to linear as the events log consisted of a large number of invocations.

The overall computational complexity level of using WebTime to analyze RUBBoS was constant whereas the complexity to analyze RUBBoS through measurement was linear. This is due to the fact that WebTime only required one run of RUBBoS to generate the events $\log$ and also only one time to transform the events log to construct the LQN model of RUBBoS. By solving this LQN model the service times of all the tasks of the system were determined and both the software and hardware bottlenecks were identified. This model could then be iteratively modified and solved until the best model, and as a result the best system set up, for optimum achievable performance behaviours is 
obtained in a very short period of time. To achieve the same results using measurement, multiple runs of RUBBoS, each taking 20 minutes would be required. Furthermore, if software or hardware needs modification, substantially more time would be required to generate results.

The accuracy of the performance prediction of WebTime can be traded off with the required post-mortem processing power. The accuracy of the results depends upon the percentage of the events which are captured by the instrumentation system. However, instrumenting everything in the system will lead to a higher volume of events log to be processed which will require more time.

WebTime in its current state can be applied only to distributed systems which are designed using the client-server paradigm. Furthermore, instrumentation for generation of an events $\log$ is performed manually and requires availability of the source code of a system.

\subsection{Future directions}

The automation of the instrumentation process is the final step needed to close the loop to automate the whole events log generation and model construction systems. Aspect oriented programming [53], byte-code instrumentation [10][94][95][96], virtual machine instrumentation such as Valgrind [97], and kernel instrumentation such as Dtrace [98], Kerninst [99] and LTT (Linux Trace Toolkit) [100] are promising approaches for this purpose.

An accurate CPU-time measurement mechanism of an event will increase the accuracy of the models created by WebTime. Currently, WebTime is dependent upon 
Java management factory class [66] which showed a consistent level of accuracy to determine the net CPU-time of events in the tests which were run in this work. However, this accuracy is undetermined and a full evaluation of this class and other technologies, especially those which are based on counting the number of the bytecodes of an event, such as ByCounter [101], will increase the accuracy of service time determination of WebTime.

The fact that the timestamp of WebTime is a logical clock may trigger the idea that WebTime can be used in applications other than construction of a performance model. For example, it could be seen as a means to realize the visualization of distributed system execution and also algorithms for debugging purposes.

WebTime uses a two-step transformation approach from events log into the LQN model with the execution graph as an intermediate model. This feature allows use of WebTime in a model driven architecture (MDA) paradigm [102] [103] in which the performance related behaviours of a software system are derived from its software design model, such as UML, in the development cycle. The performance prediction approaches which are created based on the MDA paradigm, build an intermediate model, the same way as WebTime does. This property allows for the translation of the MDA based approaches, such as PUMA[62] and KLAPER (Kernel LAnguage for PErformance and Reliability analysis) [104], by transforming their intermediate models into the execution graph of WebTime. 


\section{References}

[1] “Application Performance Management Survey," Compuware, Oct. 2006.

[2] R. Jain, The Art of Computer Systems Performance Analysis. New York: John Wiley \& Sons, 1991.

[3] C. U. Smith, L. G. Williams, and R. Lane, "Best Practices for Software Performance Engineering," in Int. CMG Conference, 2003, pp. 83-92.

[4] E. D. Lazowska, J. Zahorjan, G. Graham, and K. Sevcik, Quantitative System Performance. Engelwook Cliffs, N.J.: Printice-Hall, 1983.

[5] H. D. Mills, Top Down Programming in Large Systems. Debugging Techniques in Large Systems. Englewood Cliffs, N.J.: Prentice Hall, 1971.

[6] A. K. Shuja and J. Krebs, IBM Rational Unified Process Reference and Certification Guide: Solution Designer (RUP). Reading, MA, USA: IBM Press, 2007, p. 336.

[7] P. Kruchten, The Rational Unified Process: An Introduction. Reading, MA, USA: Addison Wesley Longman, 2003, p. 336. 
[8] H. Kameda, J. Li, C. Kim, and Y. Zhang, Optimal Load Balancing in Distributed Computer Systems. Springer, 2011.

[9] G. Franks, D. Petriu, M. Woodside, J. Xu, and P. Tregunno, "Layered Bottlenecks and Their Mitigation," in Proceedings of the 3rd international conference on the Quantitative Evaluation of Systems (QEST '06), 2006, pp. $103-114$.

[10] D. Becker, F. Wolf, W. Frings, M. Geimer, B. J. N. Wylie, and B. Mohr, "Automatic Trace-Based Performance Analysis of Metacomputing Applications," 2007 IEEE International Parallel and Distributed Processing Symposium, pp. 1$10,2007$.

[11] G. Franks, T. Al-Omari, M. Woodside, O. Das, and S. Derisavi, "Enhanced Modeling and Solution of Layered Queueing Networks," IEEE Transactions on Software Engineering, vol. 35, no. 2, pp. 148-161, Mar. 2009.

[12] C. E. Hrischuk, "Trace-Based Load Characterization for Automated Development of Software Performance Models," Carleton University, 1998.

[13] C. E. Hrischuk, C. M. Woodside, J. A. Rolia, and R. Iversen, "Trace-Based Load Characterization for Generating Performance Software Models," . IEEE Transactions on Software Engineering,, vol. 25, no. 1, pp. 122-135, 1999.

[14] C. Fidge, "Logical time in distributed computing systems," Computer, vol. 24, no. 8, pp. 28-33, 1991. 
[15] F. Mattern, "Virtual time and global states of distributed systems," in Parallel and Distributed Algorithms, 1989, pp. 215-226.

[16] “RUBBoS." [Online]. Available: http://jmob.ow2.org/rubbos.html. [Accessed: 12Oct-2012].

[17] R. Pooley, "Software engineering and performance: a roadmap," in the conference on The future of Software engineering, 2000, pp. 189-199.

[18] “OMG Unified Modeling Language TM ( OMG UML ), Super structure," Object Management Group (OMG), 2009.

[19] "UML Profile for Schedulability, Performance, and Time Specification," Object Management Group (OMG), Version 1.1, Jan. 2005.

[20] "UML Profile for Modeling and Analysis of Real-Time and Embedded systems (MARTE)," Object Management Group (OMG), Version 1.1, Jun. 2011.

[21] D. Petriu and M. Woodside, "Software performance models from system scenarios in use case maps," in Computer Performance Evaluation: Modelling Techniques, 2002, pp. 141-158.

[22] R. J. A. Buhr and R. S. O. Casselman, Use Case Maps for Object-Oriented Systems. Engelwook Cliffs, N.J.: Prentice Hall, 1996, p. 302. 
[23] “jucmnav." [Online]. Available:

http://jucmnav.softwareengineering.ca/ucm/bin/view/UCM/WebHome. [Accessed: 12-Oct-2012].

[24] P. Kähkipuro, "UML based performance modeling of object-oriented distributed systems," in Software Performance Prediction extracted from Designs, One Day Workshop, 1999.

[25] P. Kähkipuro, "The Method of Decomposition for Analyzing Queuing Networks with Simultaneous Resource Possessions," in CNDS'99) - Communications Networks and Distributed Systems Modeling and Simulation Conference The Society for Computer Simulations International, 1999.

[26] J. López-Grao, J. Merseguer, and J. Campos, "From UML activity diagrams to Stochastic Petri Nets: application to software performance engineering," in WOSP '04 - workshop on Software and performance, 2004, pp. 25-36.

[27] S. Ghosh, Distributed Systems - An Algorithmic Approach. Chapman \& Hall/CRC, 2007.

[28] G. Coulouris, J. Dollimore, T. Kindberg, and G. Blair, Distributed Systems: Concepts And Design, Fifth Edition. Addison Wesley, 2011, p. 772.

[29] L. Lamport, "Time, clocks, and the ordering of events in a distributed system," Communications of the ACM, vol. 21, no. 7, pp. 558-565, Jul. 1978. 
[30] R. Schwarz and F. Mattern, "Detecting causal relationships in distributed computations: In search of the holy grail," Distributed Computing, vol. 7, no. 3, pp. 149-174, Mar. 1994.

[31] D. Becker, R. Rabenseifner, F. Wolf, and J. C. Linford, "Scalable timestamp synchronization for event traces of message-passing applications," Parallel Computing, vol. 35, no. 12, pp. 595-607, Dec. 2009.

[32] C. Fetzer and M. Raynal, "Elastic vector time," Distributed Computing Systems, vol. 8, no. 2, pp. 103-139, 2003.

[33] S. L. Peterson and P. Kearns, "Rollback Based on Vector Time," in 12th Symp. Reliable Distributed Systems, 1993, pp. 68-77.

[34] S. Peterson and P. Kearns, "Termination detection using causality and vector time," in Southeastcon '96. "Bringing Together Education, Science and Technology"., Proceedings of the IEEE, 1996, pp. 657-660.

[35] F. Kerschbaum and J. Vayssiere, "Privacy-preserving logical vector clocks using secure computation techniques," in International Conference on Parallel and Distributed Systems (ICPADS'07), 2007, pp. 1-8.

[36] M. Ahuja, T. Carlson, and a. Gahlot, "Passive-space and time view: vector clocks for achieving higher performance, program correction, and distributed computing," IEEE Transactions on Software Engineering, vol. 19, no. 9, pp. 845-855, 1993. 
[37] S. J. Turner, "Batch based cancellation: a rollback optimal cancellation scheme in time warp simulations," in 18th Workshop on Parallel and Distributed Simulation, 2004. PADS 2004., 2004, pp. 78-86.

[38] P. a. S. Ward and D. J. Taylor, "A hierarchical cluster algorithm for dynamic, centralized timestamps," in Proceedings 21st International Conference on Distributed Computing Systems, 2001, pp. 585-593.

[39] M. Singhal and A. Kshemkalyani, "An efficient implementation of vector clocks," Information Processing Letters, vol. 43, pp. 47-52, 1992.

[40] C. Sun and W. Cai, "Capturing causality by compressed vector clock in real-time group editors," in Proceedings 16th International Parallel and Distributed Processing Symposium, 2002, pp. 558-565.

[41] F. J. Torres-Rojas and M. Ahamad, "Plausible clocks: constant size logical clocks for distributed systems," Distributed Computing, vol. 12, no. 4, pp. 179-195, Sep. 1999.

[42] R. Baldoni and M. Raynal, "Fundamentals of distributed computing: A practical tour of vector clock systems," IEEE Distributed Systems Online, vol. 3, no. 2, 2002.

[43] C. Fidge, "A limitation of vector timestamps for reconstructing distributed computations," Information processing letters, vol. 68, no. 2, pp. 87-91, 1998. 
[44] T. Israr, M. Woodside, and G. Franks, "Interaction tree algorithms to extract effective architecture and layered performance models from traces," Journal of Systems and Software, vol. 80, no. 4, pp. 474-492, Apr. 2007.

[45] M. Geimer, F. Wolf, B. Wylie, and B. Mohr, "Scalable parallel trace-based performance analysis," in Recent advances in parallel virtual machine and message passing interface, 2006, pp. 303-312.

[46] B. Mohr and F. Wolf, "KOJAK-A tool set for automatic performance analysis of parallel programs," in Euro-Par 2003 Parallel Processing, 2003, pp. 1301-1304.

[47] “OpenMP.” [Online]. Available: http://openmp.org/wp/. [Accessed: 12-Oct-2012].

[48] A. Malony and S. Shende, "Performance technology for complex parallel and distributed systems," Distributed and Parallel Systems From Instruction Parallelism to Cluster Computing. Proc. 3rd Workshop on Distributed and Parallel Systems, DAPSYS 2000, pp. 37-46, 2000.

[49] S. S. Shende, "The Tau Parallel Performance System," International Journal of High Performance Computing Applications, vol. 20, no. 2, pp. 287-311, May 2006.

[50] A. Knüpfer, H. Brunst, and J. Doleschal, "The Vampir performance analysis toolset," in Tools for High Performance Computing, 2008, pp. 139-155. 
[51] M. Snir, S. Otto, S. Huss-Lederman, D. Walker, and J. Dongarra, MPI- The Complete Reference, Volume 1. The MPI Core, Second Edition. MIT Press, Cambridge, MA, 1998.

[52] M. Rohr, A. van Hoom, and J. Matevska, "Kieker: Continuous monitoring and on demand visualization of Java software behavior," in IASTED Int'l Conf. on Software Engineering, 2008, pp. 80-85.

[53] G. Kiczales, J. Lamping, and A. Mendhekar, "Aspect-oriented programming," in ECOOP '97 - Object-Oriented Programming, 1997, pp. 220-242.

[54] G. Kiczales, E. Hilsdale, and J. Hugunin, "An overview of AspectJ," in ECOOP Object-Oriented Programming, 2001, pp. 327-353.

[55] M. Dessi, Spring 2.5 Aspect Oriented Programming. Packet Publishing, 2009.

[56] M. Woodside, "Tutorial introduction to layered modeling of software performance," Carleton University, Edition 3.0, (http://www.sce.carleton.ca/rads/lqns/lqn-documentation/), May 2002.

[57] F. J. W. Symons, "Introduction to numerical Petri nets, a general graphical model of concurrent processing systems," Australian Telecommunication Research, vol. 14, no. 1 , pp. 28-33. 
[58] J. A. Begstra, A. Ponse, and S. A. Smolka, "Handbook of Process Algebra," Elsevier Science Publishers, 2001.

[59] J. A. Rolia and K. C. Sevcik, "The Method of Layers," IEEE Transactions on Software Engineering, vol. 21, no. 8, pp. 689-700, 1995.

[60] G. Franks et. al., "Performance analysis of distributed server systems," in Software Quality, 1996, pp. 15-26.

[61] C. M. Woodside, S. Member, J. E. Neilson, and D. C. Petriu, "The Stochastic Rendezvous Network Model for Performance of Synchronous Client-Server-like Distributed Software," IEEE Trans. Computers, vol. 44, no. I, pp. 20-34, 1995.

[62] M. Woodside, D. C. Petriu, D. B. Petriu, H. Shen, T. Israr, and J. Merseguer, "Performance by unified model analysis (PUMA)," Proceedings of the 5th international workshop on Software and performance - WOSP '05, pp. 1-12, 2005.

[63] G. Franks and M. Woodside, "Effectiveness of early replies in client-server systems," Performance Evaluation, vol. 37, pp. 165-183, 1999.

[64] G. Franks, "Performance Analysis of Distributed Server Systems," PhD. thesis, Carleton University, 2000. 
[65] G. Franks, P. Maly, and M. Woodside, "Layered Queueing Network Solver and Simulator User Manual," Department of System and Computer Engineering, Carleton University (http://www.sce.carleton.ca/rads/lqns/LQNSUserMan.pdf), 2012.

[66] J. S. Perry, Java Management Extensions. O'Reilly Media, 2002.

[67] "Uncommons Maths." [Online]. Available: http://maths.uncommons.org. [Accessed: 12-Oct-2012].

[68] M. Matsumoto and T. Nishimura, "Mersenne twister: a 623-dimensionally equidistributed uniform pseudo-random number generator," ACM Transactions on Modeling and Computer Simulation, vol. 8, no. 1, pp. 3-30, Jan. 1998.

[69] TCP/IP Sockets in Java: Practical Guide for Programmers, Second Edition. Morgan Kaufmann, 2008, p. 176.

[70] Y. Fain, “Chapter 17. Java Serialization," in Java® Programming 24-Hour Trainer, Wrox, 2011 , p. 504.

[71] M. Naftalin and P. Wadler, "14.3. BlockingQueue," in Java Generics and Collections, O’Reilly Media, 2006, p. 288.

[72] J. Xu, M. Woodside, and D. Petriu, "Performance Analysis of a Software Design using the UML Profile for Schedulability, Performance and Time," in Evaluation, 
Proc. 13th International Conference on Modeling Techniques and Tools for Computer Performance, 2003, pp. 1-18.

[73] G. Lianhua, Li Franks, "Performance modeling of systems using fair share scheduling with Layered Queueing Networks," in 2009 IEEE International Symposium on Modeling, Analysis \& Simulation of Computer and Telecommunication Systems, 2009.

[74] J. E. Neilson, "PARASOL : A Simulator for Distributed and for Parallel Systems," Carleton University, Ottawa, 1991.

[75] B. Goetz, T. Peierls, J. Bloch, J. Bowbeer, D. Holmes, and D. Lea, Java Concurrency in Practice. Addison-Wesley Professional, 2006, p. 384.

[76] C. Olston, A. Manjhi, C. Garrod, A. Ailamaki, B. M. Maggs, and T. C. Mowry, “A Scalability Service for Dynamic Web Applications," in Proceedings of the Conference on Innovative Data Systems Research (CIDR) Conference, 2005, pp. $56-69$.

[77] W. Zhao and H. Schulzrinne, "DotSlash: Handling Web Hotspots at Dynamic Content Web Sites," in Proceedings IEEE INFOCOM 2006. 25TH IEEE International Conference on Computer Communications, 2006, pp. 1-5. 
[78] S. Sivasubramanian, G. Pierre, and M. Van Steen, "GlobeCBC : Content-blind Result Caching for Dynamic Web Applications," Vrije Universiteit, Technical report IR-CS-022, 2006.

[79] C. Plattner, G. Alonso, and M. T. Ozsu, "DBFarm : A Scalable Cluster for Multiple Databases," in Proceedings of the International Conference on Middleware (Middleware '06), 2006, pp. 180-200.

[80] W. Zhao and H. Schulzrinne, "DotSlash : Providing Dynamic Scalability to Web Applications with On-demand Distributed Query Result Caching," Department of Computer Science, Columbia University, Technical Report CUCS-035-05, 2005.

[81] D. Jayasinghe, S. Malkowski, Q. Wang, J. Li, P. Xiong, and C. Pu, "Variations in Performance and Scalability When Migrating n-Tier Applications to Different Clouds," in 2011 IEEE 4th International Conference on Cloud Computing, 2011, pp. $73-80$.

[82] "slashdot." [Online]. Available: http://slashdot.org. [Accessed: 12-Oct-2012].

[83] E. Jendrock, I. Evans, D. Gollapudi, K. Haase, and C. Srivathsa, The Java EE 6 Tutorial: Basic Concepts (4th Edition). Addison-Wesley Professional, 2010, p. 600.

[84] J. Hunter, Java Servlet Programming. O’Reilly Media, 1998, p. 526. 
[85] A. Vukotic and J. Goodwill, Apache Tomcat 7. Apress, 201 1, p. 293.

[86] P. DuBois, MySQL (3rd Edition). Sams Publishing, 2005, p. 1320.

[87] G. Reese, Database Programming with JDBC \& Java (2nd Edition). O'Reilly Media, 2000, p. 350 .

[88] E. R. Harold, "Chapter 15. The URLConnection Class," in Java Network Programming (Second Edition), O'Reilly Media, 2000, p. 760.

[89] E. R. Harold, “Chapter 7. Retrieving Data with URLs," in Java Network Programming (Second Edition), O'Reilly Media, 2000, p. 760.

[90] A. Mizan and G. Franks, “An automatic trace based performance evaluation model building for parallel distributed systems," in ICPE '11 Proceedings of the second joint WOSP/SIPEW international conference on Performance engineering, 2011, vol. 39 , no. 3 , pp. $61-72$.

[91] A. Mizan and G. Franks, "Automated Performance Model Construction Through Event Log Analysis," in ICST-2012 IEEE Fifth International Conference on Software Testing, Verification and Validation, 2012, pp. 636-641.

[92] A. Mizan and G. Franks, "Automatic Performance Model Construction of a Parallel Distributed Software System," in SpringSim, SCS, 2011, pp. 1-2. 
[93] A. Mizan and G. Franks, “Automated Construction of Performance Models for High Performance Distributed Applications," 12th IEEE/ACM International Symposium on Cluster, Cloud and Grid Computing (ccgrid 2012), pp. 676-677, May 2012.

[94] M. Karaorman and J. Freeman, “jMonitor: Java Runtime Event Specification and Monitoring Library," Electronic Notes in Theoretical Computer Science, vol. 113, pp. 181-200, Jan. 2005.

[95] M. Dahm, "Byte Code Engineering," in Proceedings of JIT'99, 1999, pp. 267-277.

[96] E. Bruneton, R. Lenglet, and T. Coupaye, “ASM: a code manipulation tool to implement adaptable systems," Adaptable and Extensible Component Systems, 2002.

[97] N. Nethercote and J. Seward, "Valgrind: A Program Supervision Framework," Electronic Notes in Theoretical Computer Science, vol. 89, no. 2, pp. 44-66, 2003.

[98] B. Cantrill, "Dynamic instrumentation of production systems," USENIX Annual Technical Conference, 2004.

[99] A. Tamches and B. P. Miller, "Fine-Grained Dynamic Instrumentation of Commodity Operating System Kernels Commodity Operating System Kernels," in Oper. Syst. Des. Implement, 1999, pp. 117-130. 
[100] M. Desnoyers and M. Dagenais, "LTTng: Tracing across execution layers, from the Hypervisor to user-space," in Linux Symposium, 2008, p. 101.

[101] M. Kuperberg and M. Krogmann, "ByCounter: Portable Runtime Counting of Bytecode Instructions and Method Invocations," sdqweb.ipd.kit.edu, pp. 3-8, 2011.

[102] C. Atkinson and T. Kuhne, "Model-driven development: a metamodeling foundation," Software, IEEE, vol. 20, no. 5, pp. $36-41,2003$.

[103] V. Cortellessa, D. Informatica, U. Aquila, A. Di Marco, P. Inverardi, and U. Aquila, "Software Performance Model-Driven Architecture," in ACM symposium on Applied computing (SAC '06), 2006, pp. 1218-1223.

[104] V. Grassi and R. Mirandola, "Klaper: An intermediate language for model-driven predictive analysis of performance and reliability," in The Common Component Modeling Example, 2008, pp. 327-356. 